\title{
Carnegie Mellon University
}

Doctoral Thesis

\section{Deeply Virtual Compton Scattering on the Proton in Hall A at Jefferson Lab}

Author:

Alexa Noelle Stefanko
Supervisor:

Gregg Franklin, PhD

Thesis Committee:

Brian Quinn, PhD

Curtis Meyer, $\mathrm{PhD}$

Reinhard Schumacher, PhD

Diana Parno, PhD

Julie Roche, PhD

A thesis submitted in fulfillment of the requirements for the degree of Doctor of Philosophy

Department of Physics

November 25, 2020 


\section{Acknowledgements}

I first and foremost need to express my appreciation and gratitude to my advisor Gregg Franklin, without whom this thesis would not have been possible. I could not have hoped for a more supportive and understanding advisor to help me achieve this goal, especially under the unusual circumstance of finishing this degree out of state. Gregg spared no time nor effort (even while on his retirement RV trips) to help improve this document, and me as a physicist. I am lucky to have had the opportunity to work with Gregg.

Second, I need to express my love and thanks to my family. I experienced what was the biggest personal difficulty in my life while in the middle of this degree, and without the support and love from my parents, sisters, uncles, aunts, and cousins, this thesis may not have been possible.

Next, I would like to thank the DVCS collaboration. Julie Roche and Carlos Munoz Camacho were extremely helpful in helping me understand DVCS and the analysis needed to produce this thesis. Maxime Defurne especially fielded many of my emails, and also spared no effort in responding with helpful advice and knowledge. I have also greatly enjoyed working with my fellow PhD students in the collaboration, Frederic Georges, Bishnu Karki, Salina Ali, Mongi Dlamini, and Hashir Rashad. I'd like to thank especially Bishnu for being so helpful with the troubling DIS analysis, and Hashir for being such a help with debugging problems with my DVCS fitting scripts.

I would like to thank all of my colleagues and friends from Carnegie Mellon. I am especially grateful to Larisa Thorne, with whom I shared the adventure of living in Newport News, VA and taking shifts at Jefferson Lab (before she abandoned us all for Germany (:)). It was a pleasure sharing an office with her and Juan Carlos Cornejo, and spending time with the rest of the medium energy grad students and post-docs.

I made some of my greatest friends while in graduate school, with some of the brightest and kindest people I have ever met. I enjoyed(?) sharing the dungeon office with Tori Merten and Evan Tucker, and I will never forget our desperate struggles to finish our E\&M homework. I am also grateful to Jake Fallica, and the five miserable days we spent trying to figure out what Quantum Optomechanics was, and how we would ever pull off the successful

pronunciation of micro-toroidal oscillator. I will never forget the countless nights of playing board games and watching Games of Thrones with these three people and their partners, and their friendship was invaluable during this time in my life. Without their support and friendship, finishing this degree would not have been nearly as happy of a time in my life. 


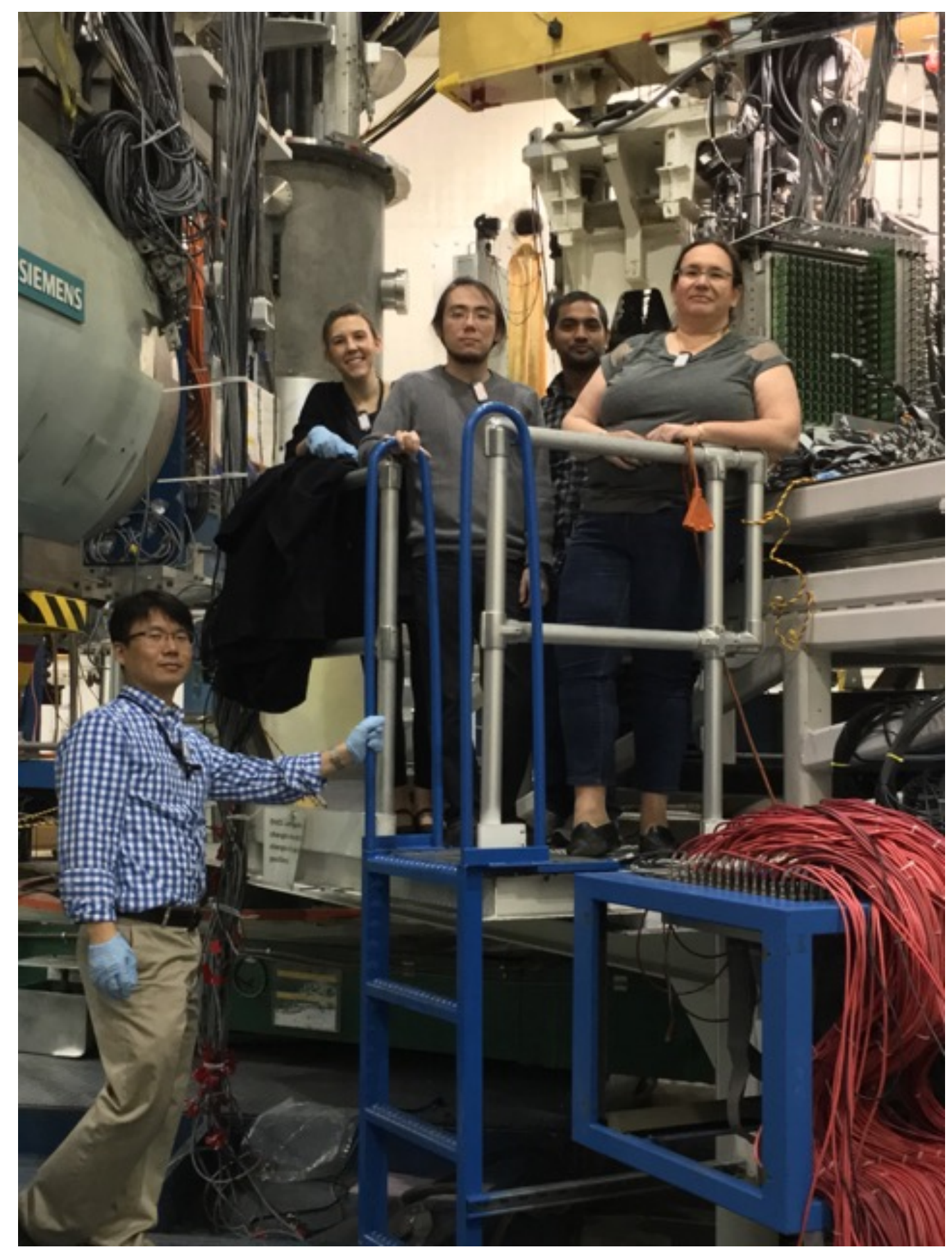




\section{Abstract}

The structure of nucleons as they are composed of quarks and gluons is not well understood. Non-perturbative methods (such as lattice-QCD) describing quark/gluon interactions are ongoing in development, but still cannot fully reproduce the structure of hadrons. Among the observables which describe longitudinal momentum and transverse position of partons, generalized parton distributions (GPDs) describe the correlations between the two, and are accessible through $p\left(\vec{e} ; e^{\prime} \gamma\right) p$ scattering experiments in the Bjorken limit when factorization holds. A complete understanding of these functions will help us to better understand the structure of nucleons.

Experiment E12-06-114 was performed by JLab's DVCS Hall A Collaboration in 2014 and 2016. Using a polarized electron beam and a liquid hydrogen target, the collaboration measured cross sections for the exclusive reaction $p\left(\vec{e} ; e^{\prime} \gamma\right) p$ at $x_{B}=0.36,0.48$, and 0.60, $3.2 \leq Q^{2} \leq 9.00$, and $-1.28 \leq t \leq-0.69$. This dissertation details the analysis and results of measuring the $p\left(\vec{e} ; e^{\prime} \gamma\right) p$ cross section at $x_{B}=0.48$. The measured cross section results allow us to demonstrate the validity of factorization in our kinematic regime, and extract Compton Form Factor combinations important for understanding GPDs. Additionally, the cross section results are used to test existing GPD models, KM10a and KM15. 


\section{Contents}

\begin{tabular}{lr}
\hline Introduction & 7
\end{tabular}

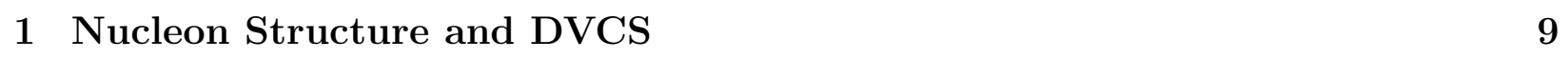

1.1 Elastic Scattering and Form Factors . . . . . . . . . . . . . . . . . . . 10

$1.2 \quad$ Deep Inelastic Scattering and Parton Distribution Functions . . . . . . . . . . . 12

1.2 .1 Bjorken Limit . . . . . . . . . . . . . . . . . . . 13

1.3 Deeply Virtual Compton Scattering and Generalized Parton Distributions . . 15

1.3.1 Virtual Compton Scattering and Photon Helicity Dependent Compton Form Factors . . . . . . . . . . . . . . . . . . . 15

1.3 .2 DVCS, the Handbag Diagram, and GPDs . . . . . . . . . . . . . 17

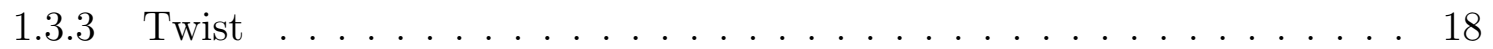

1.3 .4 GPDs and Proton Structure . . . . . . . . . . . . . . . . . . . . 19

1.3 .5 Accessing GPDs via DVCS and Factorization . . . . . . . . . . . . . . . . . 20

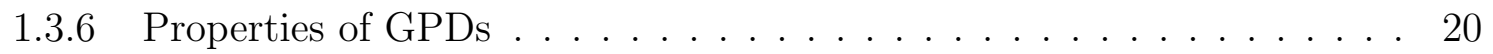

1.3 .7 DVCS and the Bethe-Heitler process . . . . . . . . . . . . . . . . . . . . . . . . . . . . . . . .

1.3 .8 Parametrization of the Bethe-Heitler amplitude . . . . . . . . . . . . . . . . . . . . . . . . . . . . . . . . .

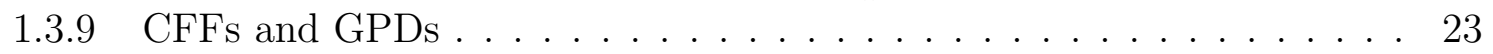

1.3 .10 Parametrization of the DVCS amplitude . . . . . . . . . . . . . 25

1.3 .11 Parametrization of the DVCS-BH interference term . . . . . . . . . . . . . . 26

1.3 .12 Accessing GPDs in the E12-06-114 Experiment . . . . . . . . . . . . . . 26

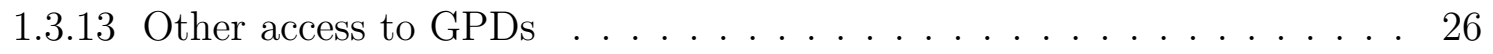

1.4 Worldwide experimental status . . . . . . . . . . . . . . . 27

$1.4 .1 \quad$ DESY Experiments . . . . . . . . . . . . . . . . . . . . . . . . . . . . . . . . . . . . . . . .

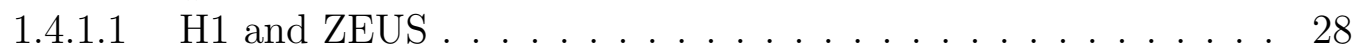

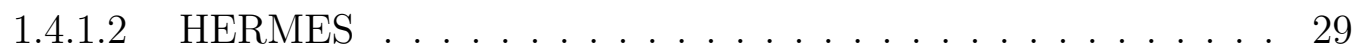

$1.4 .2 \quad$ CERN Experiment $\ldots \ldots \ldots \ldots \ldots \ldots \ldots$

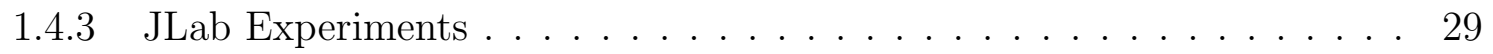

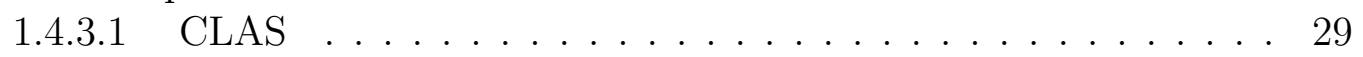

1.4 .3 .2 Hall A . . . . . . . . . . . . . . . . . . . . 29

1.4 .3 .3 Fits to CFF $\mathcal{H} \ldots \ldots \ldots \ldots \ldots$

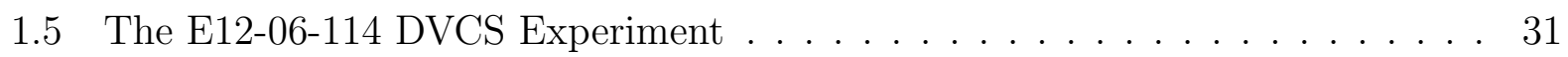

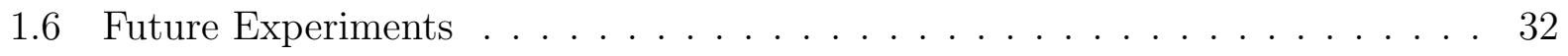


2 Jefferson Lab and Hall A Instrumentation 34

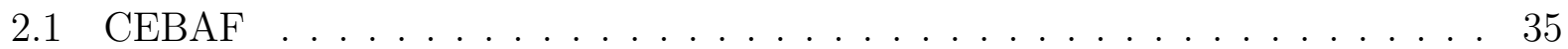

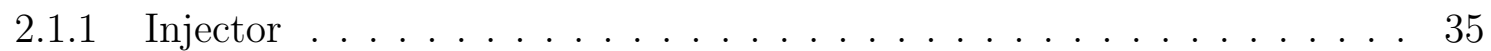

2.1 .2 Accelerator and $12 \mathrm{GeV}$ Upgrade $\ldots \ldots \ldots \ldots$

2.2 Hall A Instrumentation . . . . . . . . . . . . . . . . . . . . . . . 36

2.2 .1 Beamline . . . . . . . . . . . . . . . . . . . . 37

2.2.1.1 Polarimeters. . . . . . . . . . . . . . . . . . 37

2.2.1.1.1 Moller Polarimeter . . . . . . . . . . . . . . . 37

2.2.1.1.2 Compton Polarimeter. . . . . . . . . . . . . 37

2.2 .1 .2 Beam Energy Monitors . . . . . . . . . . . . . . . . 38

$2.2 .1 .3 \quad$ Beam Position Monitors . . . . . . . . . . . . . . . . . . . . 39

2.2.1.4 Beam Current Monitors . . . . . . . . . . . . . . . . . . 39

2.2 .2 Target $\ldots \ldots \ldots \ldots \ldots \ldots \ldots$

2.2 .3 High Resolution Spectrometers . . . . . . . . . . . . . . . . . . . . . . 39

2.2 .3 .1 Design Standards . . . . . . . . . . . . . . . . . . 40

2.2 .3 .2 Detector package . . . . . . . . . . . . . . . . . 40

2.2 .3 .3 Particle Identification . . . . . . . . . . . . . . . . . 41

2.2 .3 .4 Particle tracking . . . . . . . . . . . . . . . . 42

$2.2 .3 .5 \quad$ Scintillators . . . . . . . . . . . . . . . . . . . . 43

\begin{tabular}{|ll}
3 & Setup for E12-06-114 Experiment 44
\end{tabular}

3.1 Kinematic Settings . . . . . . . . . . . . . . . . . . . . . . . . 44

3.2 Calorimeter . . . . . . . . . . . . . . . . . . . . . 45

3.3 Data Acquisition and Triggers . . . . . . . . . . . . . . . . . . . 46

3.3.1 S0/S2M Timing and Missing Events . . . . . . . . . . . . . . . 48

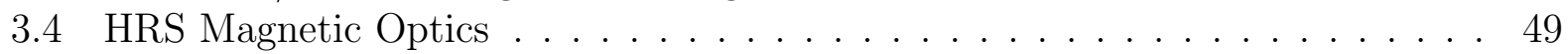

3.4 .1 Spring 2016 Optics $\ldots \ldots \ldots \ldots$. . . . . . . . . . . . . . . . . 50

3.4 .2 Fall 2016 Optics $\ldots \ldots \ldots \ldots \ldots \ldots$. . . . . . . . . . . . . . 51

3.4 .3 Acceptance and R-Functions . . . . . . . . . . . . . . . 52

3.4.3.1 R-Functions . . . . . . . . . . . . . . . . . . 52

3.4.3.1.1 Deciding on an ideal R-Cut . . . . . . . . . . 57

\begin{tabular}{|lll}
4 & The Experimental DIS Cross Section & 59
\end{tabular}

4.1 Extracting the DIS Cross Section $\ldots \ldots \ldots \ldots \ldots \ldots$

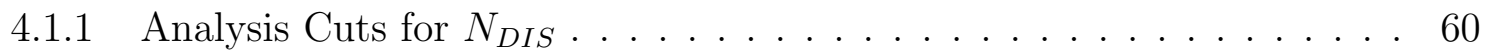

4.1.1.1 Trigger Cuts and Event Scaling . . . . . . . . . . . 60

$4.1 .1 .2 \quad$ Target vertex $\ldots \ldots \ldots \ldots \ldots$. . . . . . . . . . . . . 64

$4.1 .1 .3 \quad$ Particle identification . . . . . . . . . . . . . . . . . . . 65

4.1.1.4 Particle Tracking . . . . . . . . . . . . . . . . . . . . 67

$4.1 .1 .5 \quad$ Acceptance $\ldots \ldots \ldots \ldots \ldots$. . . . . . . . . . . . 67

$\begin{array}{lllll}4.1 .2 & \text { Luminosity } \mathcal{L} & \ldots & \ldots & \ldots\end{array} \ldots \ldots \ldots$

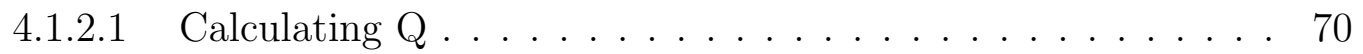

4.1 .3 Efficiency and Deadtime correction $\eta_{e x p} \ldots \ldots \ldots \ldots . \ldots . \ldots 72$ 
4.1.3.1 Deadtime correction $\eta_{D T} \ldots \ldots \ldots \ldots$. . . . . . . . . . . . . . . . . . . . . . . 73

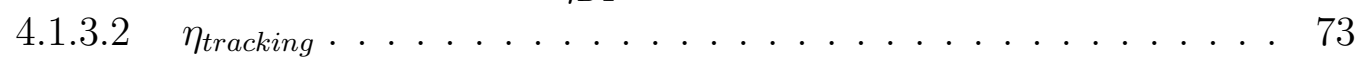

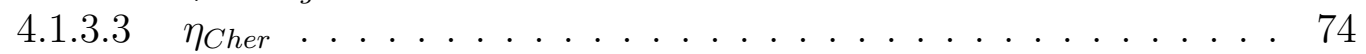

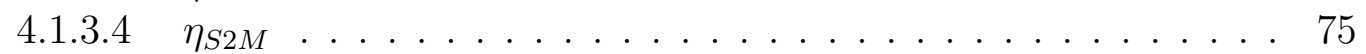

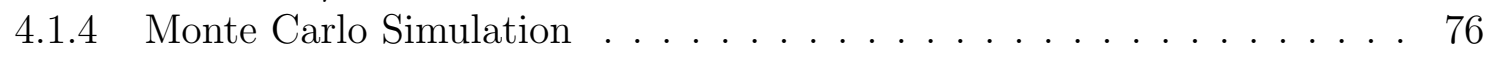

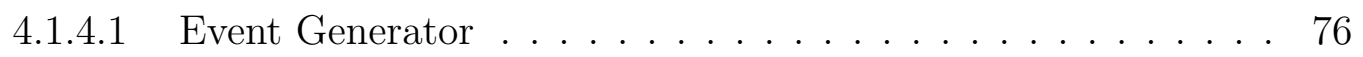

4.1.4.2 External Radiative Energy Losses . . . . . . . . . . . . . . . . . 78

4.1.4.3 Internal Radiative Energy Losses . . . . . . . . . . . . . . . . . 79

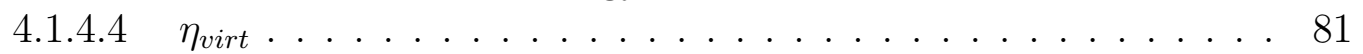

$4.1 .4 .5 \quad$ Non-physical Events . . . . . . . . . . . . . . . . . . . . . . . . . . . . . . . . . . . . . . 82

$4.1 .4 .6 \quad$ Radiative Effects on $N_{D I S} \ldots \ldots \ldots \ldots$. . . . . . . . . . . . . . . . . . . . 82

4.1.4.6.1 Radiative correction $\alpha \ldots \ldots$. . . . . . . . . . . . . . 83

4.1.4.6.2 Phase space correction $\Gamma_{D I S} \ldots \ldots \ldots$. . . . . 83

4.1.4.7 Calculating $\alpha$ and $\Gamma_{D I S} \ldots \ldots \ldots \ldots \ldots$. . . . . . . 84

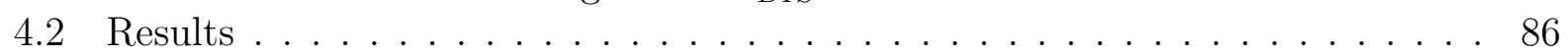

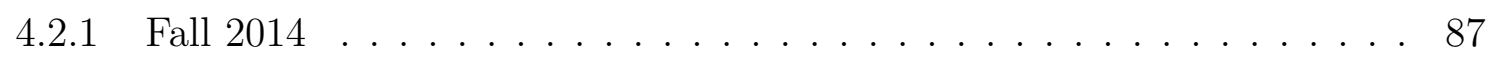

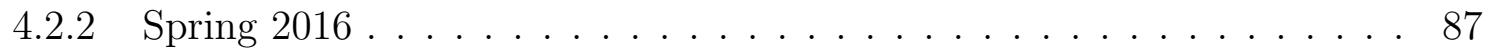

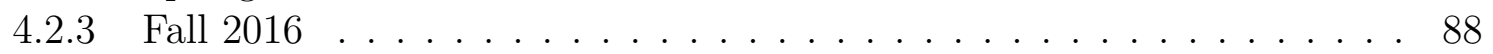

5 The DVCS Data Analysis $\quad 90$

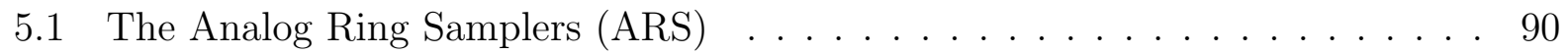

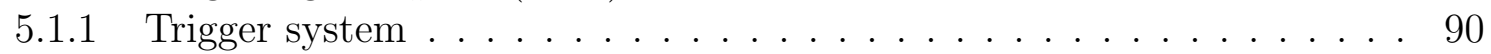

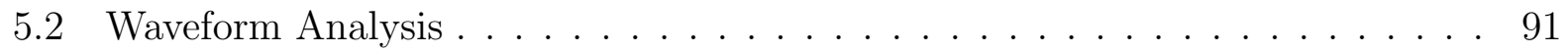

5.2 .1 Baseline Determination . . . . . . . . . . . . . . . . . . . . . . . . . . . . . . . . .

$5.2 .2 \quad$ Single-pulse fit . . . . . . . . . . . . . . . . . . . . . . . 91

$5.2 .3 \quad$ Two-pulse fit . . . . . . . . . . . . . . . . . . . . . . . . . 92

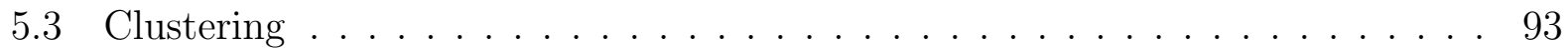

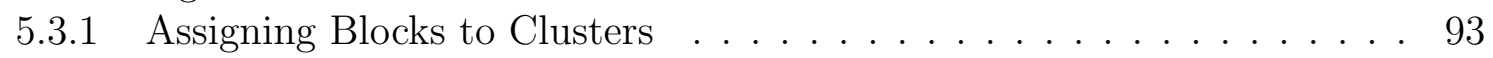

5.3 .2 Photon Reconstruction . . . . . . . . . . . . . . . . . . . 96

5.3 .3 Calorimeter Calibration . . . . . . . . . . . . . . . . . . . . . . . . . . . . . . . . .

$5.3 .3 .1 \quad$ Elastic Calibration . . . . . . . . . . . . . . . . . 97

$5.3 .3 .2 \pi^{0}$ Calibration $\ldots \ldots \ldots \ldots \ldots$

5.4 Analysis Cuts . . . . . . . . . . . . . . . . . . . . . 99

5.4 .1 Leptonic Arm Cuts . . . . . . . . . . . . . . . . . . . . . . . . . 99

5.4 .2 Real Photon Cuts . . . . . . . . . . . . . . . . . . . . . . 99

5.4 .2 .1 Accidentals Subtraction . . . . . . . . . . . . . . . . . . . . . . . . . . . . . . . . . . . . . . . . . .

$5.4 .2 .2 \pi^{0}$ Substraction . . . . . . . . . . . . . . . . . 100

5.4 .2 .3 Photon Position Cuts . . . . . . . . . . . . . . . . . 102

5.4 .3 Missing Mass Cut . . . . . . . . . . . . . . . . . . . . . . . . . . . . . . . . . .

5.4 .3 .1 SIDIS and Resonance Contamination . . . . . . . . . . . . . . . 104

5.4 .3 .1 .1 SIDIS . . . . . . . . . . . . . . . 104

5.4 .3 .1 .2 Resonances . . . . . . . . . . . . . . . 105

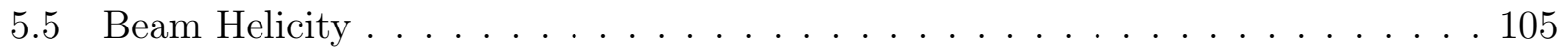


5.6 Geant4 Simulation . . . . . . . . . . . . . . . . . . . 107

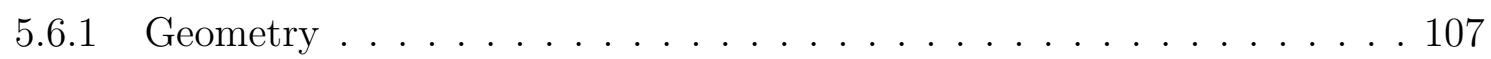

5.6 .2 Event Generator . . . . . . . . . . . . . . . . . . . . . . . . . 108

5.6 .3 Simulation Smearing/Calibration . . . . . . . . . . . . . . . . 111

5.6.3.1 Local Smearing Procedure . . . . . . . . . . . . . . . . . 112

6 The DVCS cross section extraction 114

6.1 Compton Form Factor Combination (CFFC) parameterization of the DVCS

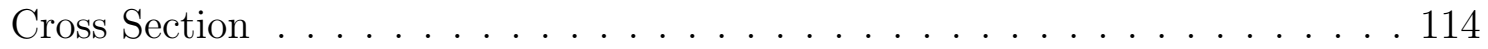

6.1 .1 Harmonic Expansion Coefficients . . . . . . . . . . . . . . . . . . 114

6.1 .2 Choice of CFFCs . . . . . . . . . . . . . . . . 115

6.2 Principle of the Extraction $\ldots \ldots \ldots \ldots \ldots \ldots$

6.2 .1 Binning Formalism . . . . . . . . . . . . . . . . . 118

$6.2 .2 \quad$ Experimental Number of Events . . . . . . . . . . . . . . . . . . . 119

6.2 .3 Fitting the CFFCs . . . . . . . . . . . . . . . . . . . 119

6.2 .4 Fitting Procedure . . . . . . . . . . . . . . . . . . . 120

6.2 .4 .1 Bethe-Heitler Subtraction . . . . . . . . . . . . . . . . 120

6.2.4.2 Calculation of the matrix $\mathcal{K}$. . . . . . . . . . . 120

6.2 .5 CFFC Extraction . . . . . . . . . . . . . . . . . 121

6.2 .6 Cross Section Reconstruction . . . . . . . . . . . . . . . . . . . . . . . . . . . . . . . . . . . . . . . . .

6.2.6.1 Choice of CFFCs $\mathcal{N} \ldots \ldots \ldots$. . . . . . . . . . . 122

6.2 .7 Results . . . . . . . . . . . . . . . . . . . . 122

6.2 .7 .1 KM Models . . . . . . . . . . . . . . . . . . . . 128

6.3 Missing Mass Cuts and Other Systematic Uncertainties . . . . . . . . . . . . 128

6.3 .1 Systematic Uncertainties . . . . . . . . . . . . . . . . . . 131

6.3 .2 Impact of DIS Results on DVCS . . . . . . . . . . . . . . . . . . . . . . . . . . . . . . . . . . .

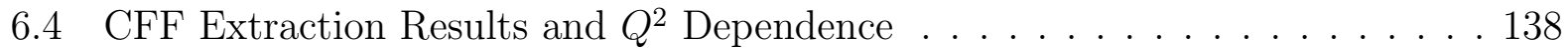

\begin{tabular}{llr}
\hline 7 Conclusion & 149
\end{tabular}

\begin{tabular}{|l|l|}
\hline A Helicity CFFs and the DVCS Cross Section 151 & 152
\end{tabular}

A.1 Definition of Variables . . . . . . . . . . . . . . . . . . . . . . . 152

A.2 The DVCS amplitude term . . . . . . . . . . . . . . . . . 153

A.3 The DVCS-BH Interference term . . . . . . . . . . . . . . . . . . . 153

\begin{tabular}{|l|l|}
\hline B Unpolarized and helicity-dependent DVCS cross sections & 157
\end{tabular}

B.1 Unpolarized Cross Sections . . . . . . . . . . . . . . . . . . . . 157

B.2 Helicity-Dependent Cross Sections . . . . . . . . . . . . . . . . . . 162

\begin{tabular}{lr}
\hline C Fitted Number of Events & 166
\end{tabular}

\begin{tabular}{|l|l|l|l|l|l|}
\hline Tables of unpolarized DVCS cross sections & 175
\end{tabular}

D.1 Unpolarized Cross Sections . . . . . . . . . . . . . . . . . . . . . . . . 175 
\begin{tabular}{lr}
\hline E Tables of Polarized DVCS cross sections & 180
\end{tabular}

E.1 Polarized Cross Sections . . . . . . . . . . . . . . . . . . . . . . . . 180

\begin{tabular}{llr}
\hline F & Acceptance Cuts & 185
\end{tabular} 


\section{List of Figures}

1.1 Diagram depicting the elastic scattering reaction. . . . . . . . . . . . 10

1.2 Deep Inelastic scattering diagram (left) and the DIS reaction in the Bjorken

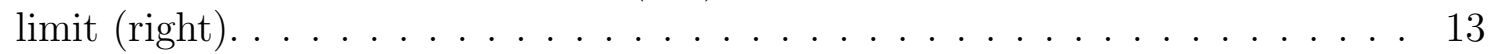

1.3 Results of measuring $F_{2}$ for a large range in $x_{B}$ and $Q^{2}$. Scaling violation is seen for large and small values of $x_{B} \ldots \ldots \ldots \ldots \ldots \ldots$

1.4 Virtual Compton Scattering (left) and the Deeply Virtual Compton Scattering handbag diagram (right). The definitions for variables in this figure can be found in the paragraph above. . . . . . . . . . . . . . 17

1.5 The angle $\phi$, expressed as $\phi_{\gamma \gamma}$ in this document, as it's defined between the

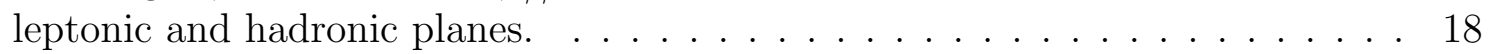

1.6 The DVCS handbag diagram. . . . . . . . . . . . . . . . . . . . . 20

1.7 DVCS vs. the Bethe-Heitler process. Note that in the Bethe-Heitler, the final state photon arises from Bremsstrahlung radiation of either the incoming or outgoing electron, and not a struck quark. . . . . . . . . . . . . 23

1.8 The kinematic regimes of worldwide DVCS effort. Figure from [46]. The grey and tan shaded regions show the $x, Q^{2}$ reach of an electron-ion collider for

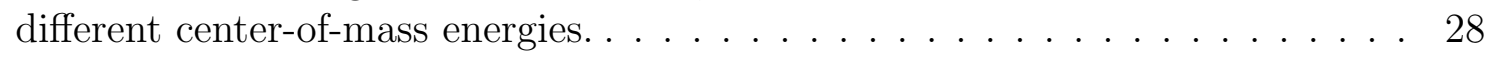

1.9 The results of the E00-110 experiment [49], showing unpolarized (top) and helicity-dependent (bottom) cross section measurements for $x_{B}=0.36, Q^{2}=$ $2.3 \mathrm{GeV}^{2}$, and $-t=0.32 \mathrm{GeV}^{2}$. The colored bands are fitted combinations of Compton Form Factors that parameterize the experimentally extracted cross section. The DVCS and Int. terms appearing in the legend refer to which term of the cross section the CFF combination was extracted from, either the DVCS amplitude, or the interference term. The twist-3 contribution in each

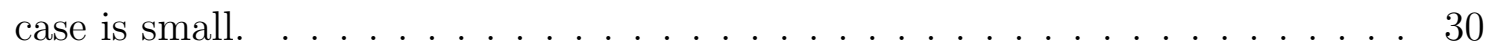

1.10 The kinematic region spanned by the proposed E12-06-114 DVCS experiment. Note that the exact beam energy values used in the experiment vary from those outlined in this figure, extracted from the proposal [48]. . . . . . . . . . . . . . 31

2.1 A figure depicting the electron accelerator facility and its upgrade at Jefferson

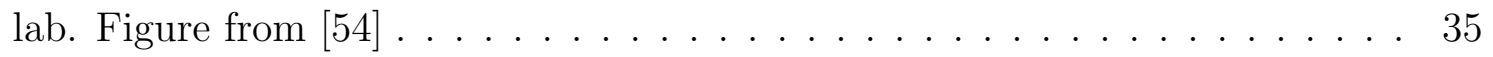

2.2 A schematic view of beamline components in Hall A. . . . . . . . . . . . . . 36

$2.3 \quad$ A figure depicting the Moller Polarimeter in Hall A at Jefferson Lab. . . . . . 37 
2.4 A figure depicting the Compton Polarimeter in Hall A at Jefferson Lab. . . . . 38

2.5 A figure depicting the left and right high resolution spectrometers in Hall A at Jefferson Lab. . . . . . . . . . . . . . . . . . . . . . . . 40

$2.6 \quad$ A schematic of the detector package for the left HRS. Note that the S2 scintillator plane is referred to in this document as the S2M, the scintillator plane sitting two meters downstream of the S1 plane. . . . . . . . . . . . . . . . . . 41

2.7 A schematic of the pion rejector. . . . . . . . . . . . . . . 42

2.8 Figures showing the side (left) and top (right) views of the vertical drift cham-

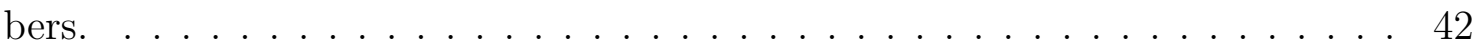

$2.9 \quad$ A figure depicting the result of a particle passing through the VDC. . . . . . . 43

$3.1 \quad$ A picture showing the photon calorimeter used in the DVCS experiment. . . . 46

3.2 An example of the number of events recorded for each trigger combination in approximately one hour of data taking. Note that the S1 was not used during the E12-06-114 experiment, so no events appear for all S1 combinations and the prescale is listed as not available (NA). Because single S0, S2M, and Cher signals do not form triggers themselves, the prescale is noted as NA. The "missing" qualifier for the S0 \& Cer DIS combination will be discussed in

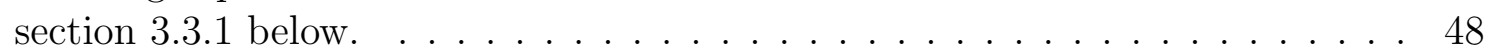

3.3 The HRS Optics Matrix . . . . . . . . . . . . . . . . . . . . . . . . . . 49

3.4 Figure (a) shows a depiction of the sieve plate used for optics calibration, and a reconstruction of data taken with the sieve in place. Figure (b) shows data

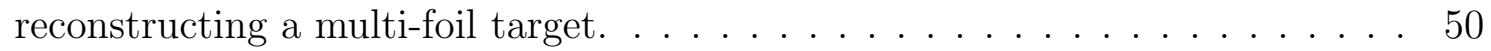

3.5 Plot from Bishnu Karki showing foil reconstruction before (blue) and after (red) optics matrix corrections for kinematic 363. . . . . . . . . . . . . 52

$3.6 \quad$ Figures showing HRS data before and after 1D acceptance cuts (red) have been applied to the four target variables $\left\{y_{t g}(\mathrm{~m}), \theta_{t g}, \phi_{t g}, \delta p\right\} . \ldots \ldots \ldots$

3.7 A figure showing the four 2D acceptance planes and the 12 cuts used to defined the R-Function for this kinematic setting. . . . . . . . . . . 55

3.8 Figures showing HRS data in target variables $\left\{y_{t g}(\mathrm{~m}), \theta_{t g}, \phi_{t g}, \delta p\right\}$ before and after an R-Cut with a positive value has been applied. . . . . . . . . . . 57

4.1 Two figures showing the effects of scaling (a.) vs. not scaling (b.) "missing" DIS events by the S0 \& Cer prescale. For this kinematic setting, not scaling the events shows good agreement with simulation data (figure 4.2 ). . . . . . . . 61

4.2 The $\delta \mathrm{p}$ distributions for the simulated data (grey) and experimental data (blue) for kinematic 484. The top figure shows the experimental data after including the missing events, without scaling by the S0 \& Cer prescale. The bottom figure shows the experimental without the missing events included. . . 62

4.3 Two figures showing the effects of scaling (a.) vs. not scaling (b.) "missing" DIS events by the S0 \& Cer prescale. For this kinematic setting, scaling the events does show good agreement with simulation data. . . . . . . . . . . 63 
4.4 Production $\mathrm{LH}_{2}$ data overlaid with data from an empty dummy target, used to identify the contamination from target cell windows. Here the blue histogram shows the dummy target, and the brown shows the standard $\mathrm{LH}_{2}$ target. The black histograms estimate the location of the cell windows and their contamination on the experimental data . . . . . . . . . . . . . . 64

4.5 Figure (a.) shows the particle identification spectra for the pion rejector layer 1 (blue) and 2 (red), and figure (b.) shows the particle identification spectra for the Cherenkov detector. These spectra are from data before any cuts have

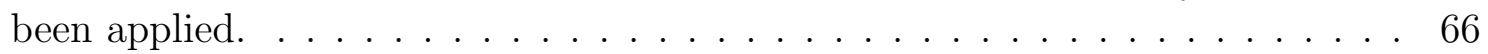

4.6 Figure (a.) shows the particle identification spectra for the pion rejector layers 1 and 2 (blue and red respectively) with only a Cherenkov signal cut, and figure (b.) shows the particle identification spectra for the Cherenkov detector with only a pion rejector cut. Using either cut alone is not sufficient to eliminate all pion contamination. . . . . . . . . . . . . 66

4.7 Figure (a.) $\quad$ shows the particle identification spectra for the pion rejector layers 1 and 2 (blue and red respectively), and figure (b.) shows the particle identification spectra for the Cherenkov detector. Both pion rejector and Cherenkov cuts have been applied in both figures. . . . . . . . . . . . . . 67

4.8 Extracted cross section (Kin 483) \% change (relative to the extracted value

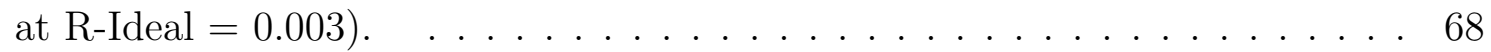

$4.9 \quad Q(t)$ vs. $t$ found from BCM scaler d1 (downstream, prescale 1 ) counts during

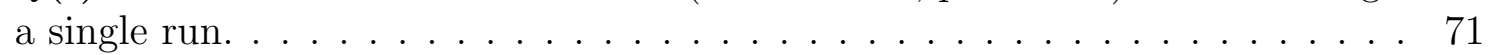

4.10 Luminosity values found for all runs of Kinematic 481. The scatter shown is related to how long the run lasted (typically anywhere between 30 minutes up

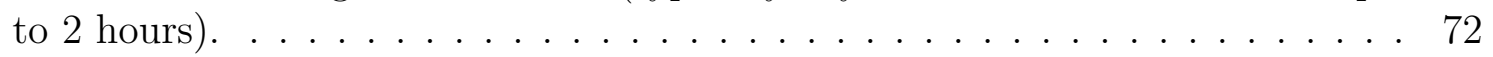

4.11 Livetime values found for all runs of Kinematic 481. . . . . . . . . . . . . . 73

4.12 Tracking correction values found for all runs for Kinematic 481. . . . . . . . . . 74

4.13 A graphic showing all energies and energy losses involved in the simulation. $\Delta E_{0}, \Delta E_{0}^{\prime}$, and $\Delta E_{4}$ are the real external radiative energy losses from traveling through the aluminum cell window and $\mathrm{LH}_{2}$ before scattering $\left(\Delta E_{0}\right.$ and $\Delta E_{0}^{\prime}$ respectively), and losses from traveling through the aluminum cell wall again after scattering $\left(\Delta E_{4}\right) . \Delta E_{1}$ and $\Delta E_{3}$ are the real internal radiative energy losses at the vertex before and after scattering. . . . . . . . . . . . . 77

4.14 The steps of event generation for the simulation. . . . . . . . . . . . . . . 78

4.15 Diagrams showing possible internal radiative energy losses. . . . . . . . . . . 80

4.16 Effects of radiative energy losses on the acceptance of the HRS. The figure on the left shows the standard $Q^{2}$ vs. $x_{b}$ phase space of the HRS acceptance, the only values the HRS can measure. The figure on the right shows the larger $Q^{2}$ vs. $x_{b}$ range that can actually be accepted by the HRS due to radiative

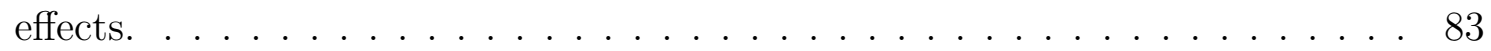

4.17 Results of extracting the DIS cross section for Kin 361. . . . . . . . . . . . . . 87

4.18 Results of extracting the DIS Cross Section for Spring 2016 Kinematics . . . . 88 
4.19 Results of extracting the DIS Cross Section for Fall 2016 Kinematics. The outlying events in Kin 362 were not found to correlate with higher current or lower tracking efficiency. The outlying set of runs in Kin 601 correlated with selecting the DIS S2M \& Cer trigger instead of the level1-DVCS S2M \& Cer trigger, whose runs did not show this discrepancy. The group of runs for Kin 603 whose measured cross section is approximately $2 \%$ larger than the rest of runs, have lower tracking efficiencies but no change in current compared to the other runs. These features were not further investigated for the sake of a timely completion of my degree. . . . . . . . . . . . . . . 89

5.1 An example of a single fitted pulse over the baseline. Figure extracted from [63] 92

5.2 An example of two fitted pulses over the baseline. Figure extracted from [63] . 93

5.3 An example of the clustering algorithm for a single photon event. . . . . . . . . 95

5.4 An example of the clustering algorithm for an event with two incident photons on nearby blocks. Image 1 shows the initial blocks passing the 2 x2 neighbor energy test. Image 2 shows the identified blocks with local maximum energies. Image 3 shows the first step of the cluster assignment, with the higher energy block taking priority for absorbing neighbors into its cluster. Image 4 shows the final step of the clustering algorithm, with all blocks assigned to a cluster. 96

5.5 Reconstructed $\pi^{0}$ invariant mass over the duration of the Fall 2016 run period. Without calibrations of the calorimeter, the apparent $\pi^{0}$ invariant mass decreases over time due to radiation damage (darkening) in the calorimeter. Image from Dr. C. Munoz Camacho. . . . . . . . . . . . . . . . . . 98

5.6 Number of events vs. coincidence time with the HRS showing the 4 ns beam

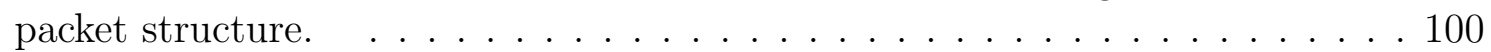

$5.7 \quad \pi^{0}$ decay in the case that photons are (c) and aren't (a) perpendicular to the boost direction. . . . . . . . . . . . . . . . . . . 101

5.8 Plot from Frédéric Georges[61]. The efficiency of the $\pi^{0}$ subtraction across the calorimeter. The ratio correctly close to 1 supports the method of $\pi^{0}$ subtraction outlined in this section. The method fails to subtract the $\pi^{0}$ contamination on the corners of the calorimeter, so an octagonal cut is used for photon position. The over-subtracted regions were found to be statistical. 102

5.9 The squared missing mass distribution for kinematic 481 after all other analysis cuts have been applied. The black histogram shows accidental experimental data before any background subtraction. The green histogram shows the background events (Section 5.4.2.1), and the blue histogram is the $\pi^{0}$ contamination (Section $\mid 5.4 .2 .2)$. The pink histogram is the $M_{X}^{2}$ distribution after background-contamination and $\pi^{0}$-subtraction. $\quad \ldots \ldots \ldots$. . . . . . 104

5.10 Beam polarization measurements as calculated for the Compton for Spring 2016, with Moller measurements. The four colored arrows show when the Moller measured beam polarization, and what value was measured. . . . . . . 106

5.11 The geometry of the DVCS Geant4 simulation. . . . . . . . . . . . . . 107 
5.12 The simulated geometry of the DVCS scattering chamber. The beam travels from the top left of the page to the bottom right. . . . . . . . . . . . . 108

5.13 Geant4 simulation steps diagram. . . . . . . . . . . . . . . . . . . . . 110

$5.14 \mu$ and $\sigma$ used to smear the simulated events interpolated from the overlapping 49 regions of the calorimeter kinematic setting 483. . . . . . . . . . . 113

6.1 Unpolarized number of simulated events (grey filled histogram), plotted with the experimental number of events (black circles) after BH subtraction for kinematic setting 481. Error bars are statistical only. . . . . . . . . . . . . 123

6.2 Unpolarized extracted cross section $\left(\times 10^{-3}\right)$ for a single $t$ bin of the kinematic setting 482, with the individual contributions from the $\mathrm{BH}$ and $\mathcal{N}=3 \mathrm{CFF}$ combinations $\left(X_{n, v} \times F_{n}\left(E,\left\langle Q^{2}\right\rangle,\left\langle x_{B}\right\rangle,\langle t\rangle, \phi_{\gamma \gamma}, \lambda\right)\right.$. The corresponding legend for this figure is in figure 6.3 below. . . . . . . . . . . . . . . 124

6.3 The legend corresponding to figure 6.2 . . . . . . . . . . . . . . . 125

6.4 Polarized extracted cross section $\left(\times 10^{-3}\right)$ for a single $t$ bin of the kinematic setting 482, with the individual contributions from the $\mathrm{BH}$ and $\mathcal{N}=2 \mathrm{CFF}$ combinations $\left(X_{n, v} \times F_{n}\left(E,\left\langle Q^{2}\right\rangle,\left\langle x_{B}\right\rangle,\langle t\rangle, \phi_{\gamma \gamma}, \lambda\right)\right.$. The corresponding legend for this figure is in figure 6.5 below $\ldots \ldots \ldots \ldots \ldots \ldots$

6.5 The legend corresponding to figure 6.4 . . . . . . . . . . . . . . 127

6.6 The smeared simulated events (teal) and the experimental number of events (grey) for kinematic setting 482. The chosen cuts for this kinematic setting are $0.45 \mathrm{GeV}^{2}$ and $1.05 \mathrm{GeV}^{2} . \ldots \ldots \ldots \ldots \ldots$. . . . . . . . . . . . . . . . . . .

6.7 The change in unpolarized extracted cross section for a single bin as the upper missing mass cut is varied. . . . . . . . . . . . . . . . 130

6.8 The \% change in unpolarized extracted cross section for all bins as the upper missing mass cut is varied by $\pm 0.06 \mathrm{GeV}^{2}$ for kinematic setting 484.

6.9 The Kin 482 unpolarized fit results without any DIS-motivated scaling. . . . . 133

6.10 The Kin 482 unpolarized fit results with the $106 \%$ DIS-motivated scaling. See figure 6.9 above for the result without scaling. . . . . . . . . . . . . . . 134

6.11 The Kin 483 unpolarized fit results without any DIS-motivated scaling. . . . . 135

6.12 The Kin 483 unpolarized fit results with the $109 \%$ DIS-motivated scaling. See figure 6.11 above for the result without scaling. $\ldots \ldots$. . . . . . . . 136

6.13 The Kin 484 unpolarized fit results without any DIS-motivated scaling. . . . . 137

6.14 The Kin 484 unpolarized fit results with the $109 \%$ DIS-motivated scaling. See figure 6.13 above for the result without scaling. . . . . . . . . . . . . 138

$6.16 Q^{2}$ dependence of the extracted twist-2 $\mathcal{C}^{D V C S}\left(\mathcal{F}_{++} \mathcal{F}_{++}^{*} \mid \mathcal{F}_{-+} \mathcal{F}_{-+}^{*}\right)$ term. Each data point has been averaged over the five vertex $t$-bins from the respective kinematic setting. The error bars are statistical. The horizontal line and corresponding uncertainty band represent the average of the 4 data points, and the propagated error associated with the average. The data points from left to right are from Kin 481, 482, 483 and 484. . . . . . . . . . . . . . . 139 
6.15 The $\mathcal{C}^{D V C S}\left(\mathcal{F}_{++} \mathcal{F}_{++}^{*} \mid \mathcal{F}_{+-} \mathcal{F}_{+-}^{*}\right)$ CFFC extracted in all 5 t-bins for each kinematic setting, as a function of $Q^{2}$. The error bars are the resulting error from the fitting procedure. The dark (green) data points are the resulting values from fitting the data without any corrections related to the DIS cross section extraction results. The light (green) data points are the results after scaling the number of events in each bin by the DIS-motivated value $(100 \%, 106 \%$, $106 \%$, and $109 \%$ for these kinematic settings in order $) . \quad \ldots . . . . . .140$

$6.18 Q^{2}$ dependence of the extracted twist-2 $\operatorname{Re} \mathcal{C}^{I}\left(\mathcal{F}_{++}\right)$CFFC. Each data point has been averaged over the five vertex $t$-bins from the respective kinematic setting. The error bars are statistical. The horizontal line and corresponding uncertainty band represent the average of the 4 data points, and the propagated error associated with the average. The data points from left to right are from Kin 481, 482,483 and 484. . . . . . . . . . . . . . . . . . . 141

6.17 The $\operatorname{Re} \mathcal{C}^{I}\left(\mathcal{F}_{++}\right)$CFFC extracted in all 5 t-bins for each kinematic setting, as a function of $Q^{2}$. The error bars are the resulting error from the fitting procedure. The dark (magenta) data points are the resulting values from fitting the data without any corrections related to the DIS cross section extraction results. The light (magenta) data points are the results after scaling the number of events in each bin by the DIS-motivated value $(100 \%, 106 \%, 106 \%$, and $109 \%$ for these kinematic settings in order $). \ldots \ldots$. . . . . . . . . . 142

6.19 The $\operatorname{Re} \mathcal{C}^{I}\left(\mathcal{F}_{0+}\right)$ CFFC extracted in all 5 t-bins for each kinematic setting, plotted vs. $Q^{2}$. The error bars are the resulting error from the fitting procedure. The dark (cyan) data points are the resulting values from fitting the data without any corrections related to the DIS cross section extraction results. The light (cyan) data points are the results after scaling the number of events in each bin by the DIS-motivated value $(100 \%, 106 \%, 106 \%$, and $109 \%$ for these kinematic settings in order $). \ldots \ldots \ldots$. . . . . . . . . . . . . . . . . .

6.20 The extracted twist-3 $\operatorname{Re} \mathcal{C}^{I}\left(\mathcal{F}_{0_{+}}\right)$CFFC. Each data point has been averaged over the five vertex $t$-bins from the respective kinematic setting. The error bars are statistical. The horizontal line and corresponding uncertainty band represent the average of the 4 data points, and the propagated error associated with the average. The data points from left to right are from Kin 481, 482, 483 and 484. . . . . . . . . . . . . . . . . . . . . . . . 144

6.21 The $\operatorname{Im} \mathcal{C}^{I}\left(\mathcal{F}_{++}\right)$CFFC extracted in all 5 t-bins for each kinematic setting, as a function of $Q^{2}$. The error bars are the resulting error from the fitting procedure. The dark (blue) data points are the resulting values from fitting the data without any corrections related to the DIS cross section extraction results. The light (blue) data points are the results after scaling the number of events in each bin by the DIS-motivated value $(100 \%, 106 \%, 106 \%$, and $109 \%$ for these kinematic settings in order $). \ldots \ldots \ldots$. . . . . . . . . . . . . . 
$6.22 Q^{2}$ dependence of the extracted twist-2 $\operatorname{Im} \mathcal{C}^{I}\left(\mathcal{F}_{++}\right)$CFFC. Each data point has been averaged over the five vertex $t$-bins from the respective kinematic setting. The error bars are statistical. The horizontal line and corresponding uncertainty band represent the average of the 4 data points, and the propagated error associated with the average. The data points from left to right are from Kin 481, 482,483 and 484. . . . . . . . . . . . . . 146

6.23 The $\operatorname{Im} \mathcal{C}^{I}\left(\mathcal{F}_{0+}\right)$ CFFC extracted in all 5 t-bins for each kinematic setting, plotted vs. $Q^{2}$. The error bars are the resulting error from the fitting procedure. The dark (aqua) data points are the resulting values from fitting the data without any corrections related to the DIS cross section extraction results. The light (aqua) data points are the results after scaling the number of events in each bin by the DIS-motivated value $(100 \%, 106 \%, 106 \%$, and $109 \%$ for these kinematic settings in order $). \ldots \ldots \ldots$. . . . . . . . . . . . . . . . . . . .

$6.24 Q^{2}$ dependence of the extracted twist-3 $\operatorname{Im} \mathcal{C}^{I}\left(\mathcal{F}_{0+}\right)$ CFFC. Each data point has been averaged over the five vertex $t$-bins from the respected kinematic setting. The error bars are statistical. The horizontal line and corresponding uncertainty band represent the average of the 4 data points, and the propagated error associated with the average. The data points from left to right are from Kin $481,482,483$ and $484 . \ldots \ldots \ldots \ldots$

B.1 Unpolarized cross sections for kinematic setting $481\left(\chi^{2} / d o f=2.03\right)$. Error bars are statistical only. The uncertainty introduced from the choice of missing mass cut is quoted for each bin in the tables of cross sections (Appendix D). . 158

B.2 Unpolarized cross sections for kinematic setting $482\left(\chi^{2} /\right.$ dof $\left.=1.35\right)$. Error bars are statistical only. The uncertainty introduced from the choice of missing mass cut is quoted for each bin in the tables of cross sections (Appendix D). .159

B.3 Unpolarized cross sections for kinematic setting $483\left(\chi^{2} / d o f=1.68\right)$. Error bars are statistical only. The uncertainty introduced from the choice of missing mass cut is quoted for each bin in the tables of cross sections (Appendix D). . 160

B.4 Unpolarized cross sections for kinematic setting $484\left(\chi^{2} / d o f=1.39\right)$. Error bars are statistical only. The uncertainty introduced from the choice of missing mass cut is quoted for each bin in the tables of cross sections (Appendix D). . 161

B.5 Helicity-dependent cross sections for kinematic setting $481\left(\chi^{2} / d o f=0.78\right)$. Error bars are statistical only. The uncertainty introduced from the choice of missing mass cut is quoted for each bin in the tables of cross sections

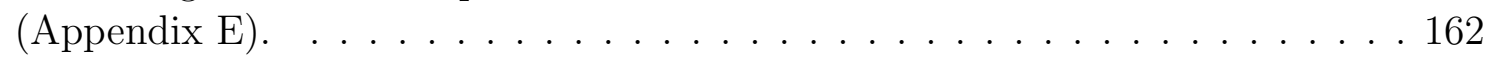

B.6 Helicity-dependent cross sections for kinematic setting $482\left(\chi^{2} /\right.$ dof $\left.=0.94\right)$. Error bars are statistical only. The uncertainty introduced from the choice of missing mass cut is quoted for each bin in the tables of cross sections

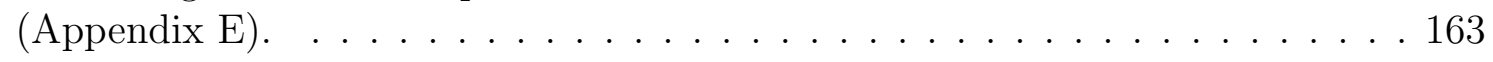


B.7 Helicity-dependent cross sections for kinematic setting $483\left(\chi^{2} /\right.$ dof $\left.=0.95\right)$. Error bars are statistical only. The uncertainty introduced from the choice of missing mass cut is quoted for each bin in the tables of cross sections

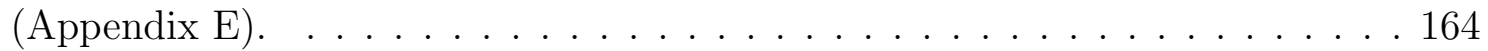

B.8 Helicity-dependent cross sections for kinematic setting $484\left(\chi^{2} /\right.$ dof $\left.=1.02\right)$. Error bars are statistical only. The uncertainty introduced from the choice of missing mass cut is quoted for each bin in the tables of cross sections

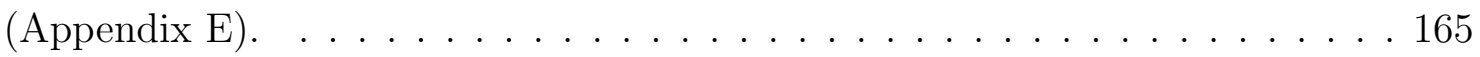

C.1 Unpolarized number of events for kinematic setting 481. The black circles represent the experimental number of events, and the grey histograms represent the fitted number of simulated events $\left(\chi^{2} / d o f=2.03\right) . \ldots \ldots 167$

C.2 Unpolarized number of events for kinematic setting 482. The black circles represent the experimental number of events, and the grey histograms represent the fitted number of simulated events $\left(\chi^{2} / d o f=1.35\right) . \ldots \ldots 8$

C.3 Unpolarized number of events for kinematic setting 483. The black circles represent the experimental number of events, and the grey histograms represent the fitted number of simulated events $\left(\chi^{2} / d o f=1.68\right) . \ldots \ldots 169$

C.4 Unpolarized number of events for kinematic setting 484. The black circles represent the experimental number of events, and the grey histograms represent the fitted number of simulated events $\left(\chi^{2} / d o f=1.39\right) . \ldots \ldots$. . . . . 170

C.5 Helicity-dependent number of events for kinematic setting 481. The black circles represent the experimental number of events, and the grey histograms represent the fitted number of simulated events $\left(\chi^{2} /\right.$ dof $\left.=0.78\right) . \ldots$. . . . 171

C.6 Helicity-dependent number of events for kinematic setting 482. The black circles represent the experimental number of events, and the grey histograms represent the fitted number of simulated events $\left(\chi^{2} / d o f=0.94\right) . \ldots$. . . . 172

C.7 Helicity-dependent number of events for kinematic setting 483. The black circles represent the experimental number of events, and the grey histograms represent the fitted number of simulated events $\left(\chi^{2} /\right.$ dof $\left.=0.95\right) . \ldots$. . . . 173

C.8 Helicity-dependent number of events for kinematic setting 484 . The black circles represent the experimental number of events, and the grey histograms represent the fitted number of simulated events $\left(\chi^{2} /\right.$ dof $\left.=1.02\right) . \ldots . . .174$ 


\section{List of Tables}

1.1 A table describing how to access GPDs through DVCS on the proton. A GPD listed in red would be kinematically suppressed in such an experiment. . . . . 19

1.2 Summary of the 11 planned kinematic settings for E12-06-114. The three fixed values of $x_{B}$ with varying $Q^{2}$ allow us to separate the real and imaginary contributions to the DVCS amplitude. . . . . . . . . . . . . 32

2.1 Design standards for an HRS . . . . . . . . . . . . . . . . . . . 40

3.1 Description of actual Kinematic Settings used for the E12-06-114 experiment. 44

3.2 PAC allocation of beam time for kinematic settings $\ldots \ldots \ldots 4$

3.3 The combination triggers used for data acquisition. . . . . . . . . . . . . . . . 47

3.4 Q1 status during Spring 2016 Running . . . . . . . . . . . . . . . . . . . . . . . . . . . . . . . 51

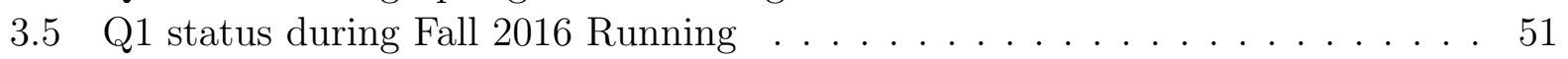

$4.1 \quad$ A table of important parameters used for extracting the DIS cross section. . . 60

4.2 A table of vertex cuts for each kinematic setting. The cuts differ for each kinematic setting because the target position changed. . . . . . . . . . . 65

$4.3 \quad$ A table of R-Errors, the R-Cut uncertainty for each kinematic setting. . . . . . 69

4.4 Important parameters used for calculation of the integrated luminosity for a

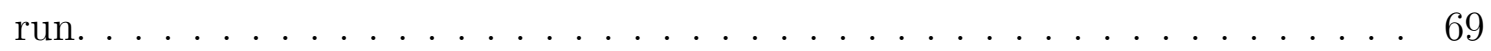

4.5 A table of experimental efficiencies for each kinematic setting. . . . . . . . . . 76

$4.6 \quad$ A table of $\eta_{v i r t}$ for each kinematic setting. . . . . . . . . . . . . . . . . 81

4.7 Results of calculating $\alpha$ and $\Gamma_{D I S}$ for all kinematic settings $\ldots \ldots$. . . . 86

4.8 A table of extracted DIS cross section results. . . . . . . . . . . . . . . 86

5.1 A table of neighboring 2x2 block array energy thresholds for events in the

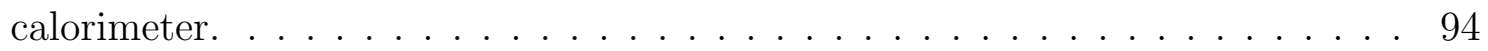

5.2 The minimum reconstructed energy the photon must have to be included as a DVCS event, compared with the expected photon energy. . . . . . . . . . . . 99 
6.1 A table of the CFFCs that can be used to parametrize the DVCS cross section. The notation $\mathcal{F}_{a b}$ follows from the helicity CFFs defined in section 1.3.1, $\mathcal{C}$ means combination, and the superscript $D V C S$ and $I$ refer to CFFCs that contribute to the pure DVCS term and the BH-DVCS interference term, respectively. The Pol/Unpol column denotes if the CFFC is accessible from the unpolarized or polarized cross section. The superscripts $A$ and $V$ relate to the vector and axial vector functions from equation 1.17 . More details on the $A$ and $V$ terms can be found in [17]. . . . . . . . . . . . . . . . 115

6.2 The normalized $\chi^{2}$ for the $x_{B}=0.48$ kinematic settings. The degrees of freedom $d o f=\mathcal{R}-\mathcal{N} \mathcal{V}$. For the unpolarized cross section fit, dof $=120-3$ $\times 5=105$. For the polarized cross section fit, $\operatorname{dof}=120-2 \times 5=110$. . . . 123

6.3 The selected missing mass cuts. . . . . . . . . . . . . . . . . . 129

6.4 Summary of systematic uncertainties. . . . . . . . . . . . . . . . . . . 131

6.5 The normalized $\chi^{2}$ for the $x_{B}=0.48$ kinematic settings with the DIS-motivated scaling, compared to without. . . . . . . . . . . . . 132

6.6 Mean CFFCs values extracted from the fit of the polarized and unpolarized DVCS cross sections. The errors listed here are the propagated error associated with the average, and not the standard deviation of the average. . . . . 148

A.1 A table of the CFFCs that can be used to parametrize the DVCS cross section. The notation $\mathcal{F}_{a b}$ follows from the helicity CFFs defined in section 1.3.1, $\mathcal{C}$ means combination, and the superscript $D V C S$ and $I$ refer to CFFCs that contribute to the pure DVCS term and the BH-DVCS interference term, retrospectively. The superscripts $A$ and $V$ relate to the vector and axial vector functions from equation 1 1.17, More details on the $A$ and $V$ terms can be

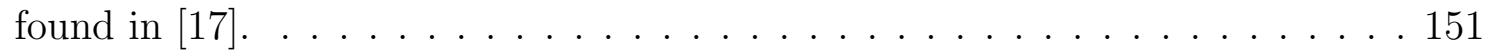

D.1 Table of unpolarized extracted cross section values $\left(\mathrm{pb} / \mathrm{GeV}^{4}\right)$ for Kin481. The \pm values are the statistical errors, and the separated + and - are the systematic error introduced from the missing mass cut. The overall systematic uncertainties from table 6.4 are not represented here $\ldots$. . . . . . . . . . 176

D.2 Table of unpolarized extracted cross section values $\left(\mathrm{pb} / \mathrm{GeV}^{4}\right)$ for Kin482. The \pm values are the statistical errors, and the separated + and - are the systematic error introduced from the missing mass cut. The overall systematic uncertainties from table 6.4 are not represented here. . . . . . . . . . . . . 177

D.3 Table of unpolarized extracted cross section values $\left(\mathrm{pb} / \mathrm{GeV}^{4}\right)$ for Kin483. The \pm values are the statistical errors, and the separated + and - are the systematic error introduced from the missing mass cut. The overall systematic uncertainties from table 6.4 are not represented here. . . . . . . . . . . . 178

D.4 Table of unpolarized extracted cross section values $\left(\mathrm{pb} / \mathrm{GeV}^{4}\right)$ for Kin484. The \pm values are the statistical errors, and the separated + and - are the systematic error introduced from the missing mass cut. The overall systematic uncertainties from table 6.4 are not represented here. . . . . . . . . . . . . . 179 
E.1 Table of helicity-dependent extracted cross section values (pb/GeV ${ }^{4}$ ) for Kin481. The \pm values are the statistical errors, and the separated + and - are the systematic error introduced from the missing mass cut. The overall systematic uncertainties from table 6.4 are not represented here. . . . . . . . . . . . . . 181

E.2 Table of helicity-dependent extracted cross section values $\left(\mathrm{pb} / \mathrm{GeV}^{4}\right)$ for Kin482. The \pm values are the statistical errors, and the separated + and - are the systematic error introduced from the missing mass cut. The overall systematic uncertainties from table 6.4 are not represented here. . . . . . . . . . . . . . 182

E.3 Table of helicity-dependent extracted cross section values $\left(\mathrm{pb} / \mathrm{GeV}^{4}\right)$ for Kin483. The \pm values are the statistical errors, and the separated + and - are the systematic error introduced from the missing mass cut. The overall systematic uncertainties from table 6.4 are not represented here . . . . . . . . . . . 183

E.4 Table of helicity-dependent extracted cross section values $\left(\mathrm{pb} / \mathrm{GeV}^{4}\right)$ for Kin 484 . The \pm values are the statistical errors, and the separated + and - are the systematic error introduced from the missing mass cut. The overall systematic uncertainties from table 6.4 are not represented here. . . . . . . . . . . . . . 184

F.1 2D Cuts for the Kinematic Settings 361 and $481 \ldots$. . . . . . . . . . . 186

F.2 2D Cuts for the Kinematic Settings 362, 363, 601, and 603 . . . . . . . . . 186

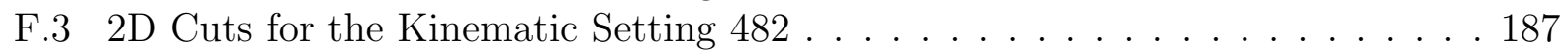

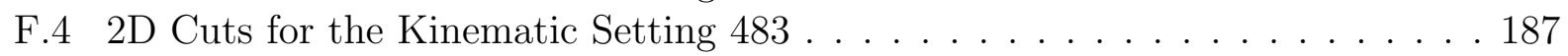

F.5 2D Cuts for the Kinematic Setting $484 \ldots \ldots$. . . . . . . . . . . . . 188 


\section{Introduction}

The curiosity of mankind has driven us to understand our universe. Sometime around 400 BC, the ancient Greek Democritus (or perhaps before him Leucippus) theorized that all of the matter in the universe was made up of atoms. Over two thousand years later, we have progressed drastically in atomic theory, and understand that ordinary (that is, not dark) matter actually is made up of atoms, which themselves are made of electrons, protons, and neutrons. Until just sixty years ago, we did not know that the proton and neutron were themselves made up of quarks and gluons, and we still do not know how these come together to produce nucleons.

The Standard Model is currently the most widely accepted theory describing matter and its interactions. It includes a finite set of elementary particles (quarks and leptons), and three of the four fundamental forces (and their mediating bosons) through which these particles interact. The four fundamental forces are gravity, electromagnetism, the weak force, and the strong force, and physicists are striving to understand how these forces work at all energy regimes. One massive goal for nuclear particle physicists is to understand the strong force well enough to fully describe nucleons using only their constituent quarks and gluons (partons).

At high momentum transfers (short distances), the strong force interaction is welldescribed with perturbative quantum chromodynamics (QCD) field theory. However, as we move to lower energies, and distances close to the size of the proton, the strong coupling constant gets large and perturbative QCD cannot be used. Non-perturbative methods (such as lattice-QCD) of describing quark/gluon interactions are ongoing in development, but still cannot fully reproduce the structure of hadrons.

By the 1990's, generalized parton distributions (GPDs) were developed, that describe quark dynamics and are accessible through scattering experiments. GPDs contain information on the correlation of transverse spatial position and longitudinal momentum of partons, and having a complete understanding of these functions will help us to better understand the strong force in the lower energy regime. Because of this, there is a worldwide effort to obtain enough experimental data to correctly parameterize GPDs. Deeply Virtual Compton Scattering (DVCS) is one of the simplest channels that gives access to GPDs. In 2004, the first DVCS experiment took place at Jefferson Lab in Newport News, Virginia.

This thesis documents Jefferson Lab's second generation DVCS experiment E12-06-114, performed by JLab's DVCS Hall A Collaboration. Using a polarized electron beam and a liquid hydrogen target, the collaboration measured cross sections for the inclusive reaction 
$p\left(\vec{e} ; e^{\prime}\right) X$ and the exclusive reactions $p\left(\vec{e} ; e^{\prime} \gamma\right) p$ and $p\left(\vec{e} ; e^{\prime} \pi^{0}\right) p$ at 9 different kinematic settings. This dissertation contains my analysis of the $p\left(\vec{e} ; e^{\prime}\right) X$ at the 9 kinematic settings and the $p\left(\vec{e} ; e^{\prime} \gamma\right) p$ at the four $x_{B}=0.48$ kinematic settings. Other combinations of cross sections and kinematic settings were analyzed by graduate students Frederic Georges, Bishnu Karki, Salina Ali, and Hashir Rashad.

My extracted cross sections for the Deep Inelastic $p\left(\vec{e} ; e^{\prime}\right) X$ are presented in section 4.2 and my extracted cross sections for $p\left(\vec{e} ; e^{\prime} \gamma\right) p$ are presented in section 6.2.7 and appendices B, C, D, and E. The Compton Form Factor Combinations (CFFCs) extracted by my fitting procedures are presented in section 6.4. Note that while this data is not sufficient to isolate individual Compton Form Factors, a future DVCS experiment planned in Hall C of JLab will acquire the data necessary to do so. These results are part of a larger program designed to test the theory of Generalized Parton Distributions.

The layout of this document is as follows:

- Chapter 1 describes Compton Form Factors and how GPDs can be accessed through the experimental DVCS cross section, and the currently favored models for their parameterization. This chapter will also detail the ongoing efforts of other experiments seeking to access GPDs.

- Chapter 2 will discuss Jefferson Lab and the experimental Hall A where the DVCS experiment took place.

- Chapter 3 focuses on the complete experimental setup of the DVCS experiment.

- Chapter 4 is dedicated to the extraction of the DIS cross section, and how the important results from this analysis may affect the DVCS cross section extraction. It contains information regarding data analysis cuts, and the DIS Monte Carlo simulation.

- Chapter 5 details the analysis used to extract the DVCS cross section. This includes the data analysis cuts used, and a description of the Geant4 DVCS simulation.

- Chapter 6 details the fitting method used to actually extract the DVCS cross section and the combinations of Compton Form Factors that parameterize it. 


\section{Chapter 1}

\section{Nucleon Structure and DVCS}

Before the late 1950's, the proton was thought to be a point-like particle. Elastic scattering experiments performed at SLAC (Stanford Linear Accelerator) around this time, however, began to show hints that nucleons have internal structure [1]. Results from deep inelastic scattering (DIS) experiments conducted in the 1960's and 70's, again at SLAC, gave direct evidence that protons and neutrons are not elementary particles, and are instead composed of point-like objects called quarks (later known generally as partons to include gluons) [2], [3]. In fact, the elastic scattering cross section can be parametrized with form factors (FFs), functions related to the transverse spatial distribution of charge (quarks) inside nucleons. Similarly, the DIS cross section can be parametrized with parton distribution functions (PDFs), that give information about the longitudinal momentum distribution of quarks inside nucleons.

For the last sixty years, FFs and PDFs have helped better our understanding of quantum chromodynamics (QCD), and are still quite relevant in solving modern problems in nuclear physics (such as the proton radius problem [5]). Unfortunately though, they do not give any information about correlations between the transverse spatial distribution of quarks and their longitudinal momenta, which are an important step towards a complete understanding of the theory. In the mid 1990's, generalized parton distributions (GPDs) were introduced as functions that could provide these correlations, encapsulating the information of both FFs and PDFs [6].

GPDs obey a set of important sum rules and properties that help theorists develop different models for them [10]. The observables of deep exclusive scattering processes give us experimental access to GPDs, and the results of such experiments can be compared to the different GPD models. A worldwide effort to collect data in all kinematic regimes is currently ongoing, with the goal to successfully model GPDs.

This chapter will discuss elastic and inelastic scattering, and the important FFs and PDFs that can be extracted from experimental observables. An extension into GPDs and their properties will follow, with a brief description of the experimental observables that can be used to extract such functions in an analogous way to elastic and inelastic scattering. An overview of the past and future worldwide effort to study GPDs will be presented, and finally, the experiment specific to discussion in this document will be introduced. 


\subsection{Elastic Scattering and Form Factors}

Elastic scattering occurs when an incident particle scatters off a target particle, and only the same particles appear in the final state. For elastic $e p \rightarrow e^{\prime} p^{\prime}$ scattering, shown in Figure 1.1, the following important variables are defined:

- $k=(E, \vec{k})$ and $k^{\prime}=\left(E^{\prime}, \vec{k}^{\prime}\right)$ the four momenta for incoming and outgoing electrons respectively

- $\theta_{e}$ the scattering angle

- $q=k-k^{\prime}$ the four momentum transfer

- $Q^{2}=-q^{2}$ called the virtuality of the photon, the negative of the 4-momentum transfer

- $p$ and $p^{\prime}$ the proton momentum before and after scattering, respectively

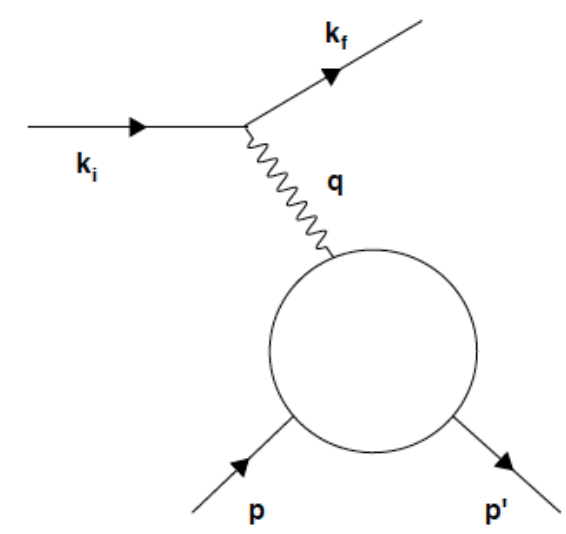

Figure 1.1: Diagram depicting the elastic scattering reaction.

The value of $Q^{2}$ determines the scale at which we probe the proton, with larger values representing probing at shorter distances. Because the final state particles are restricted to those of the initial state, we can calculate the energy of the outgoing electron using:

$$
E^{\prime}=\frac{E}{1+\frac{2 E}{M} \sin ^{2}\left(\frac{\theta_{e}}{2}\right)}
$$

The value of $Q^{2}$ for the reaction is given by:

$$
Q^{2}=4 E E^{\prime} \sin ^{2}\left(\frac{\theta_{e}}{2}\right)
$$

Prior to the 1950's SLAC experiment, that first showed evidence of internal nucleon structure, the elastic ep scattering was thought to be well-described by the Mott cross section: 


$$
\frac{d \sigma}{d \Omega}_{M o t t}=\frac{\alpha}{4 E^{2} \sin ^{4}\left(\frac{\theta_{e}}{2}\right)} \cos ^{2}\left(\frac{\theta_{e}}{2}\right)
$$

The expression of the Mott cross section assumes a point-like spin- $\frac{1}{2}$ scattering target. However, with evidence of the proton being an extended target, an additional factor had to be added to account for structure within the target:

$$
\frac{d \sigma}{d \Omega}=\left(\frac{d \sigma}{d \Omega}\right)_{M o t t}|F(\Delta)|^{2}
$$

Here, $\Delta=p-p^{\prime}=-q$ for elastic scattering; $F(\Delta)$ is called a form factor, whose Fourier transform (in the limit of low $Q^{2}$ ) is related to the spatial distribution of charge $\rho(r)$ inside the proton.

$$
F(\Delta)=\int \rho(r) e^{i \Delta r} d r^{3}
$$

For a spin $\frac{1}{2}$ nucleon, with extended structure and an anomalous magnetic moment, the Rosenbluth cross section best describes the elastic scattering in the single-photon exchange approximation as [12]:

$$
\left(\frac{d \sigma}{d \Omega}\right)_{\text {Rosenbluth }}=\left(\frac{d \sigma}{d \Omega}\right)_{M o t t}\left\{F_{1}^{2}\left(Q^{2}\right)+\frac{Q^{2}}{4 M^{2}}\left[F_{2}^{2}\left(Q^{2}\right)+2\left(F_{1}\left(Q^{2}\right)+F_{2}\left(Q^{2}\right)\right)^{2} \tan ^{2}\left(\frac{\theta}{2}\right)\right]\right\}
$$

The Pauli and Dirac form factors $F_{1}\left(Q^{2}\right)$ and $F_{2}\left(Q^{2}\right)$ respectively can be written in terms of the Sachs form factors $G_{E}$ and $G_{M}$, the Fourier transforms of charge and magnetic distributions inside the proton

$$
\begin{aligned}
& F_{1}\left(Q^{2}\right)=\frac{G_{E}\left(Q^{2}\right)+\tau G_{M}\left(Q^{2}\right)}{2 \tau} \\
& F_{2}\left(Q^{2}\right)=\frac{G_{M}\left(Q^{2}\right)-G_{E}\left(Q^{2}\right)}{1+\tau}
\end{aligned}
$$

where $\tau=\frac{Q^{2}}{4 M^{2}}$ [13]. Both $G_{E}$ and $G_{M}$ can be extracted experimentally, using a method called Rosenbluth separation, where elastic scattering is performed at two different beam energies, but $Q^{2}$ remains fixed. $G_{E}$ is still an important quantity of study. For example, its measurement over $Q^{2}$ allows us to extrapolate the value of the proton charge radius using:

$$
<r_{E}^{2}>=-\left.6 \frac{d G_{E}\left(Q^{2}\right)}{d Q^{2}}\right|_{Q^{2}=0}
$$

Different methods of finding the proton charge radius have yielded different results. Measurements made from elastic scattering experiments yield a radius of $r_{p}=0.879(8) \mathrm{fm}$. This agrees well with measurements made using the Lamb shift of electronic hydrogen, being $r_{p}=0.8768(69) \mathrm{fm}[14]$. However, both of these values are in large disagreement with the recent measurements made in 2010 using the Lamb shift of muonic hydrogen, $r_{p}=0.84184(67)$ fm [15]. This discrepancy has been resolved with results from 2019[7] and 2020[8] that show good agreement with the smaller radius. 


\subsection{Deep Inelastic Scattering and Parton Distribution Functions}

Unlike elastic scattering, the allowed particles in the final state of deep inelastic scattering (DIS) reactions are not restricted to those in the initial state. The DIS reaction of interest for this thesis is $e p \rightarrow e^{\prime} X$. In this reaction, in the single photon approximation, an incoming electron exchanges a virtual photon with a proton, resulting in an outgoing electron and a system of particles, denoted as $X$, in the final state.

The invariant mass of the hadronic final state is defined as $W^{2}=(p+q)^{2}$, and the "deep" inelastic regime is characterized as having $W>M$ and $Q^{2}>M^{2}$. Another important variable in DIS reactions is the Bjorken variable $x_{B}=\frac{Q^{2}}{2 M \nu}$, with $\nu=E-E^{\prime}$. The DIS cross section (like the elastic cross section), is parametrized by functions that encapsulate information about the internal proton structure, $F_{1}\left(x_{B}, Q^{2}\right)$ and $F_{2}\left(x_{B}, Q^{2}\right)$, and is given by

$$
\frac{d \sigma}{d \Omega d E^{\prime}}=\left(\frac{d \sigma}{d \Omega}\right)_{M o t t}\left[\frac{F_{2}\left(x_{B}, Q^{2}\right)}{\nu}+\frac{2}{M} F_{1}\left(x_{B}, Q^{2}\right) \tan ^{2}\left(\frac{\theta}{2}\right)\right]
$$

Measurements of the experimental DIS cross section at SLAC in the 1960's found that the functions $F_{1}\left(x_{B}, Q^{2}\right)$ and $F_{2}\left(x_{B}, Q^{2}\right)$ are approximately independent of $Q^{2}$ (referred to as "scaling"). This observation indicated that the electron scattered off of a point-like, spin-

$\frac{1}{2}$, particle inside the proton. Results from SLAC also showed that $F_{1}$ and $F_{2}$ obey the Callan-Gross relation

$$
F_{2}\left(x_{B}\right)=2 x_{B} F_{1}\left(x_{B}\right)
$$

that implies the point-like particles are fermions, supporting the theory laid out by GellMann (Nobel Prize 1969) in which nucleons were predicted to be made up of quarks and gluons. 

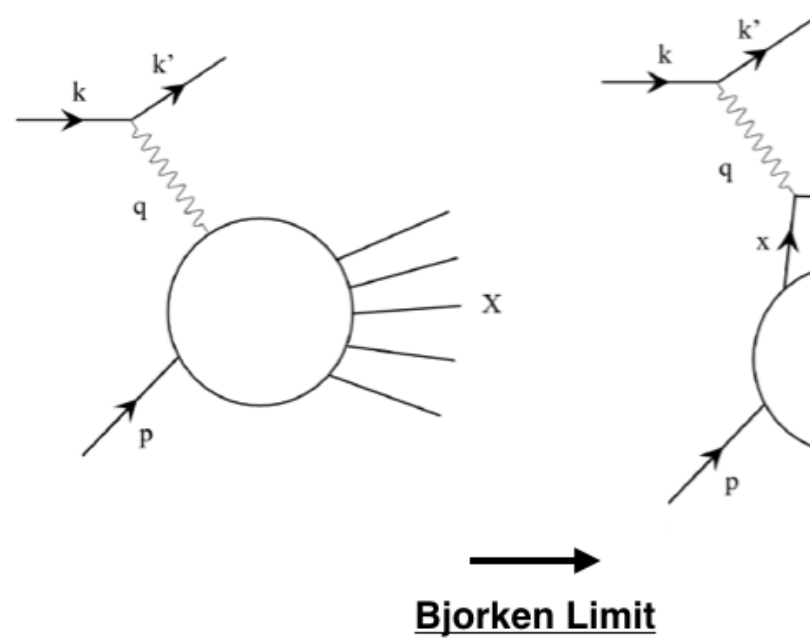

Figure 1.2: Deep Inelastic scattering diagram (left) and the DIS reaction in the Bjorken limit (right).

\subsubsection{Bjorken Limit}

In the limit where $Q^{2} \rightarrow \infty$ and $\nu \rightarrow \infty$, but $x_{B}$ remains fixed (referred to as the Bjorken limit or infinite momentum frame), the exchanged virtual photon interacts with a single quark in the proton (Figure 1.2). In this limit, $x_{B}$ can be interpreted as the fraction of the proton's total longitudinal momentum carried by the struck quark. In this limit, the function $F_{2}$ can be expressed in terms of parton distribution functions ( $\mathrm{PDFs}), q\left(x_{B}\right)$, with the equation

$$
F_{2}\left(x_{B}\right)=x_{B} \sum_{f} e_{f}^{2} q_{f}\left(x_{B}\right)
$$

with $e_{f}$ the parton charge, as a fraction of proton charge. The PDF $q_{f}\left(x_{B}\right)$ can be thought of as the probability of scattering off a parton of flavor $f$, carrying longitudinal momentum fraction $x_{B}$ of the proton. The results of measuring $F_{2}$ from various experiments worldwide can be seen in Figure 1.3 . 


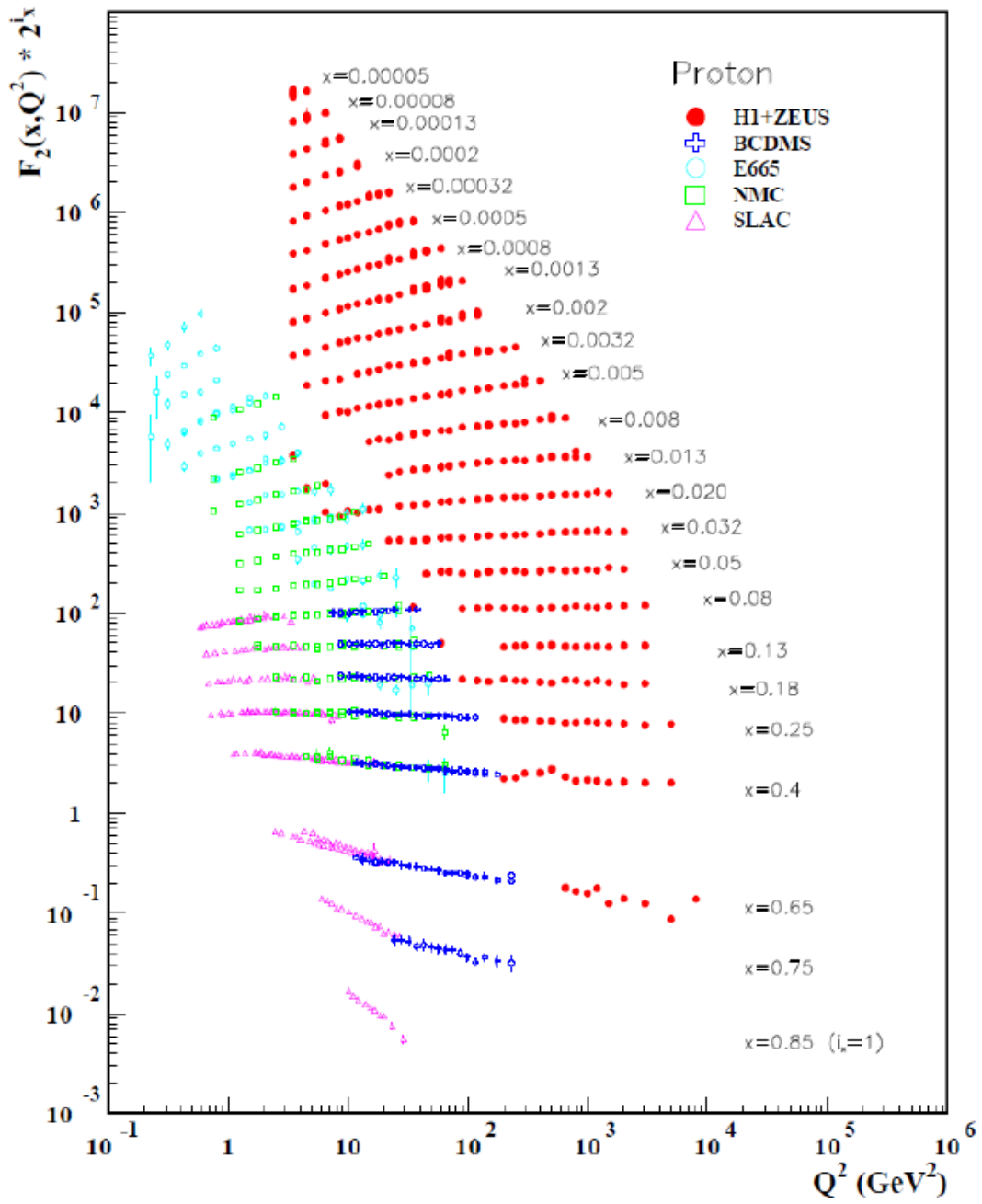

Figure 1.3: Results of measuring $F_{2}$ for a large range in $x_{B}$ and $Q^{2}$. Scaling violation is seen for large and small values of $x_{B}$.

An important observation from figure 1.3 is that for very small and large values of $x_{B}$ (proton momentum fractions) $F_{2}$ is no longer independent of $Q^{2}$. This is known as scaling violation. The DGLAP (Dokshitzer, Gribov, Lipatov, Altarelli, and Parisi) equations model the logarithmic dependence of the function $F_{2}$ in $Q^{2}$, attributing this behavior to QCD radiative effects; that is, the struck quark radiating gluons, similar to an electron radiating photons in QED. 


\subsection{Deeply Virtual Compton Scattering and General- ized Parton Distributions}

Elastic and deep inelastic scattering experiments give limited information on the internal structure of nucleons. To reiterate, form factors (FFs) describe the transverse spatial position of quarks, and parton distribution functions (PDFs) describe the longitudinal momentum distributions of quarks. However, neither of these provide " 3 -D" information on the structure of nucleons, the correlations between FFs and PDFs. Generalized parton distributions (GPDs) do contain this information, and are indirectly experimentally accessible through deeply virtual compton scattering (DVCS). Instead of measuring the GPDs directly, we instead measure functions called helicity Compton Form Factors, that relate to GPDs in the high $Q^{2}$ limit.

\subsubsection{Virtual Compton Scattering and Photon Helicity Depen- dent Compton Form Factors}

In this section, $a$ and $a^{\prime}$ will be used to denote the spin of a virtual photon with $a, a^{\prime} \in\{+, 0,-\}$, and $b$ will be used to denote the spin of a real photon with $b \in\{+,-\}$.

The hadronic vertex shown in Fig. 1.4(left) corresponds to an initial state virtual photon of helicity $a$, and a nucleon of spin $s= \pm \frac{1}{2}$, to make 6 initial basis states. The final state (real) photon of helicity $b$, and nucleon of spin $s^{\prime}= \pm \frac{1}{2}$ form 4 basis states.. The $6 \mathrm{x} 4$ matrix of amplitudes can be expressed by the sum of photon helicity dependent Compton Form Factors (helicity-CFFs), $\mathcal{H}_{a b}, \mathcal{E}_{a b}, \tilde{\mathcal{H}}_{a b}, \tilde{\mathcal{E}}_{a b}$, denoted generically as $\mathcal{F}_{a b}$, where $a$ is the incoming virtual photon helicity and $b$ the outgoing real photon helicity:

$$
\mathcal{F}_{a b}\left(Q^{2}, x_{B}, t\right) \in\left\{\mathcal{H}_{a b}, \mathcal{E}_{a b}, \tilde{\mathcal{H}}_{a b}, \tilde{\mathcal{E}}_{a b}\right\}
$$

where $Q^{2}$ and $x_{B}$ were defined in sections 1.1 and 1.2 , and $t$ is the momentum transfer to the proton $t=\Delta^{2}=\left(p-p^{\prime}\right)^{2}$. Parity conservation requires that

$$
\mathcal{F}_{--}=\mathcal{F}_{++}, \mathcal{F}_{0-}=\mathcal{F}_{0+}, \text { and } \mathcal{F}_{-+}=\mathcal{F}_{+-}
$$

so that there are only 12 , rather than 24 , independent helicity-CFFs which we choose to be $\mathcal{F}_{++} \in\left\{\mathcal{H}_{++}, \mathcal{E}_{++}, \tilde{\mathcal{H}}_{++} \tilde{\mathcal{E}}_{++}\right\}, \mathcal{F}_{0+} \in\left\{\mathcal{H}_{0+}, \mathcal{E}_{0+}, \tilde{\mathcal{H}}_{0+}, \tilde{\mathcal{E}}_{0+}\right\}$, and $\mathcal{F}_{+-} \in\left\{\mathcal{H}_{+-}, \mathcal{E}_{+-}, \tilde{\mathcal{H}}_{+-}, \tilde{\mathcal{E}}_{+-}\right\}$.

The Virtual Compton Scattering (VCS) cross section is proportional to

$$
\left|\mathcal{T}_{V C S}(s, \lambda)\right|^{2}=\frac{1}{Q^{2}} \sum_{a, a^{\prime}} \mathcal{L}_{a a^{\prime}}(\lambda, \phi) \mathcal{W}_{a a^{\prime}}(s),
$$

where $\lambda$ is the incident electron helicity, $s$ is the initial nucleon spin, and $\phi$ is the angle between the leptonic and hadronic planes (figure 1.5). $\mathcal{L}_{a a^{\prime}}$ are the well-known squared leptonic helicity amplitudes [17], and $\mathcal{W}_{a a^{\prime}}$ is the hadronic tensor. The sum over the finalstate photon helicity has been included in $\mathcal{L}_{a a^{\prime}}$ and the sum over the final-state nucleon spin 
is included in $\mathcal{W}_{a a^{\prime}}(s)$. The contraction of the lepton tensor and hadronic tensor involves summing over the virtual photon helicities, $a, a^{\prime}$.

The hadronic tensor comes from the hadronic vertex, summing the hadronic helicity amplitudes $\mathcal{T}_{a b}$ over the final-state photon helicity, $b$ :

$$
\mathcal{W}_{a a^{\prime}}=\sum_{b \in+,-} \mathcal{T}_{a b}^{(\text {had })} \mathcal{T}_{a^{\prime} b}^{(\text {had)* }}
$$

The helicity-CFFs appear as coefficients in the expansion of the hadronic helicity amplitudes in spinor notation:

$$
\mathcal{T}_{a b}^{(h a d)}=\mathcal{V}_{a b}-b \mathcal{A}_{a b}
$$

where $\mathcal{V}_{a b}$ and $\mathcal{A}_{a b}$ are vector and axial-vector functions parameterized by the helicityCFFs. The definitions of $\mathcal{A}_{a b}$ and $\mathcal{V}_{a b}$ are given in [17.

In calculating the experimentally measurable cross section, the Bethe-Heitler (see Section 1.3.7) process must be included since it is indistinguishable from VCS, as shown in figure 1.7. The experimentally measurable cross section is

$$
d^{5} \sigma \equiv \frac{d^{5} \sigma}{d Q^{2} d x_{B} d t d \phi_{\gamma \gamma} d \phi_{e}}\left(Q^{2}, x_{B}, t, \phi_{\gamma \gamma}\right)=\frac{\alpha^{3} x_{B} y^{2}}{16 \pi^{2} Q^{4} \sqrt{1+\epsilon^{2}}}\left|\frac{\mathcal{T}}{e^{3}}\right|^{2}
$$

where $\mathcal{T}=\mathcal{T}_{B H}+\mathcal{T}_{V C S}$. Here $\alpha$ is the strong constant, $y$ is the lepton energy loss $y=\frac{p \cdot q}{p \cdot k}$, $\epsilon=\frac{2 x_{B} M}{Q}$ a term accounting for target mass effects, $e$ the elementary charge, $\phi_{\gamma \gamma}$ is the angle between the leptonic and hadronic planes (Figure 1.5), and $\phi_{e}$ is the relative angle between $\phi_{\gamma \gamma}$ and the nucleon polarization vector (see figure 1 of [17] for more details on $\phi_{e}$, sometimes written as $\varphi$ ).

Because the target in this experiment is unpolarized, we can integrate over $\phi_{e}$ to get

$$
d^{4} \sigma \equiv \int d^{5} \sigma d \phi_{e}=\frac{\alpha^{3} x_{B} y^{2}}{8 \pi Q^{4} \sqrt{1+\epsilon^{2}}} \frac{1}{e^{6}}\left[\left|\mathcal{T}_{V C S}\right|^{2}+\left|\mathcal{T}_{B H}\right|^{2}+\mathcal{I}_{V C S-B H}\right]
$$

The $\left|\mathcal{T}_{V C S}\right|^{2}$ contribution will contain bi-linear combinations of the helicity-CFFs, while the interference contribution, $\mathcal{I}_{V C S-B H}$, is composed of linear combinations in the helicityCFFs. The Bethe-Heitler term, $\left|\mathcal{T}_{B H}\right|^{2}$, is determined by known quantities, and its contribution can be subtracted off from the experimentally measured cross sections. These terms will be discussed in more detail below, and in Chapter 6 and in Appendix A.

The experiment discussed in this thesis measured Deeply Virtual Compton Scattering (DVCS), i.e. VCS in the high $Q^{2}$ regime. The experiment utilized a polarized electron beam and an unpolarized target, allowing access to combinations of helicity-CFFs (rather than individual helicity-CFFs). Combinations of the helicity-CFFs will be referred to in this thesis as CFFCs. The measurements are part of a broader program designed to isolate the individual CFFs in the high $Q^{2}$ regime. 


\subsubsection{DVCS, the Handbag Diagram, and GPDs}

The DVCS reaction on the proton ${ }^{1}$ is a subset of DIS, where $e p \rightarrow e^{\prime} p^{\prime} \gamma$. In the Bjorken limit (Section 1.2.1), the incoming electron scatters off a single quark (via a virtual photon), the quark emits a real photon, and then recombines with the outgoing proton.

The process depicted in Figure 1.4 (right) involves the initial quark absorbing a virtual photon (with 4-momentum $q$ ), followed by the emission of a real photon (with 4-momentum $\left.q^{\prime}\right)$ and the reinsertion of a quark. The diagram can be evaluated with a loop-momentum integral. By setting $\bar{q}=\left(q+q^{\prime}\right) / 2$ and $\bar{p}=\left(p+p^{\prime}\right) / 2$, where $p$ and $p^{\prime}$ are the initial and final 4 -momenta of the proton, the evaluation reduces to a one dimensional integral over $x$. The relevant longitudinal momenta for the quark become $P^{+}=(x+\xi) \bar{p}$ and $P^{-}=(x-\xi) \bar{p}$.
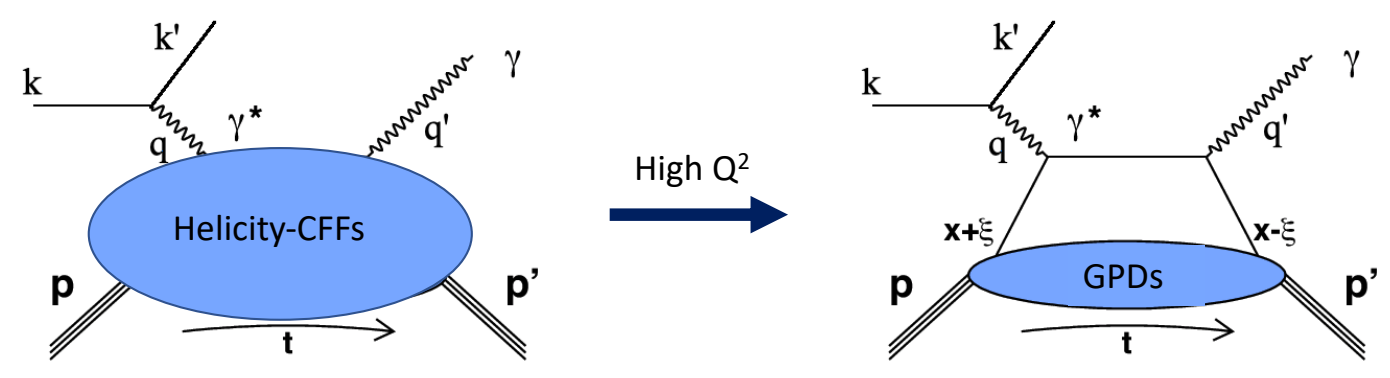

Figure 1.4: Virtual Compton Scattering (left) and the Deeply Virtual Compton Scattering handbag diagram (right). The definitions for variables in this figure can be found in the paragraph above.

Note that the squared momentum transfer to the proton is $t$, where $t=\Delta^{2}=\left(p-p^{\prime}\right)^{2}$, and $-2 \xi$ is the overall longitudinal momentum transfer to the quark. In the previous paragraph, the new variable, $\xi$, is an experimentally accessible parameter called the skewness. In the limit that $|t|<<Q^{2}$, we have that

$$
\xi=\frac{x_{B}\left(1+\frac{t}{2 Q^{2}}\right)}{2-x_{B}+x_{B}\left(\frac{t}{Q^{2}}\right)} \approx \frac{x_{B}}{2-x_{B}}
$$

\footnotetext{
${ }^{1}$ DVCS on the neutron is also an area of interest, but the experiment documented in this thesis only includes DVCS on the proton.
} 


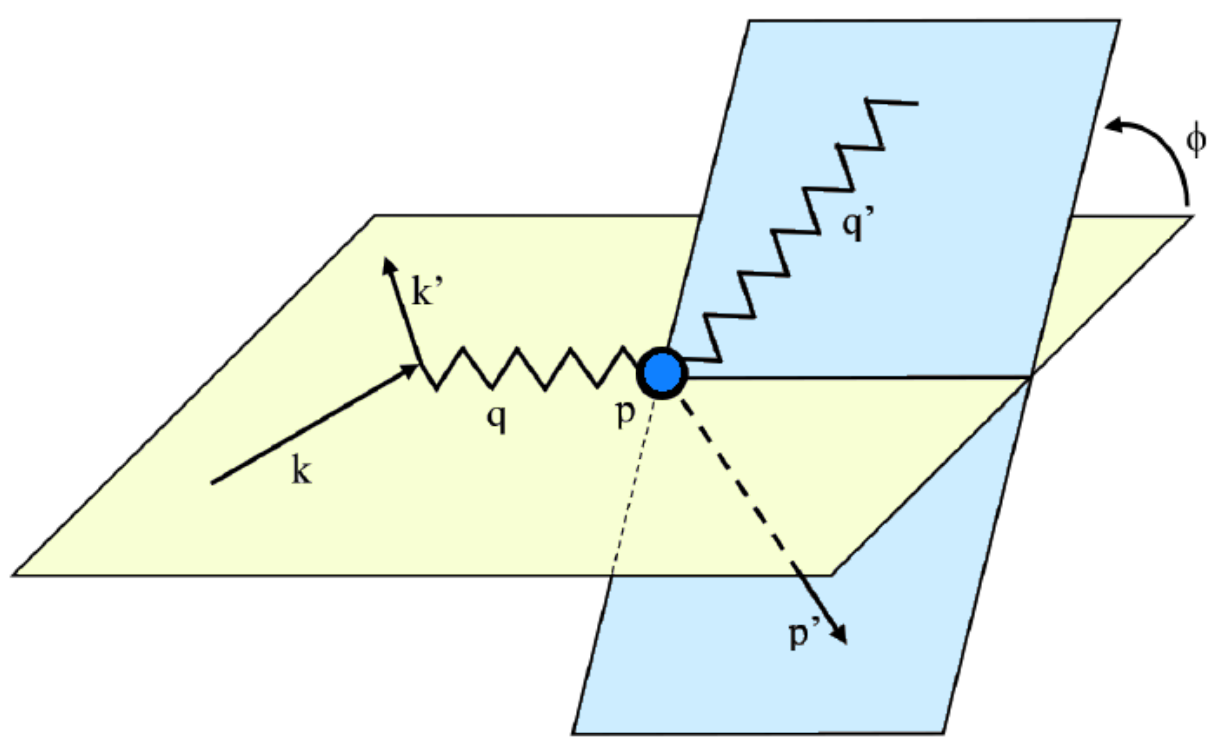

Figure 1.5: The angle $\phi$, expressed as $\phi_{\gamma \gamma}$ in this document, as it's defined between the leptonic and hadronic planes.

We define $\phi_{\gamma \gamma}$ as the angle between the leptonic plane and the hadronic plane, as shown in Figure 1.5. Mathematically, $\phi_{\gamma \gamma}$ follows the Trento convention:

$$
\begin{aligned}
& \cos \phi_{\gamma \gamma}=\frac{\vec{q} \times \vec{k}}{|\vec{q} \times \vec{k}|} \cdot \frac{\vec{q} \times \vec{p}^{\prime}}{\left|\vec{q} \times \vec{p}^{\prime}\right|} \\
& \sin \phi_{\gamma \gamma}=\frac{\left(\vec{k} \times \vec{p}^{\prime}\right) \cdot \vec{q}}{|\vec{q} \times \vec{k}|\left|\vec{q} \times \vec{p}^{\prime}\right|}
\end{aligned}
$$

\subsubsection{Twist}

The VCS amplitude (equation 1.15) can be expressed as an operator product expansion of the product of quark electromagnetic currents, where the "twist" is defined as the dimension minus the spin of the operator. For DVCS, the leading order term in the expansion is twist2. Higher order terms are suppressed by increasing powers of $\frac{1}{Q}$. Therefore, a twist-3 term is suppressed by $\frac{1}{Q}$, twist- 4 by $\frac{1}{Q^{2}}$, etc. In the limit that $Q^{2} \rightarrow \infty$, only the leading order, twist-2, term would remain. Experimentally, however, only a $Q^{2}$ of a few $\mathrm{GeV}$ is used, and higher twist-3+ may need to be considered to accurately parameterize the experimental cross section and extract information about GPDs.

The DVCS cross section has been computed in the twist-3 approximation in [17], which was used for the analysis documented in this thesis. 


\subsubsection{GPDs and Proton Structure}

GPDs describing quark dynamics parameterize nucleon structure. The twist-2 quark GPDs, for a quark of flavor $f$, are $H_{f}, \tilde{H}_{f}, E_{f}, \tilde{E}_{f}$, and depend on $x, \xi$, and $t$. These four GPDs are chiral-even, meaning the struck and reabsorbed quarks have the same helicity. Note that flavor separation is not a subject of this thesis, so the flavor subscript $f$ will often be dropped.

Twist-3 quark GPDs also exist, but their contributions to the DVCS cross section are expected to be small in the high $Q^{2}$ region relevant to this thesis. Additionally, there also exist chiral-odd, or transversity, quark GPDs $\left(H_{q T}, \tilde{H}_{q T}, E_{q T}, \tilde{E}_{q T}\right)$ for situations where the struck quark changes helicity. The DVCS channel is not sensitive to these chiral-odd GPDs, so they will not be discussed or referenced for the remainder of this thesis. Similarly, GPDs exist that describe gluon dynamics. However, in the valence quark kinematic regime of the E12-06-114 experiment, we expect our results to be insensitive to the gluon GPDs. Therefore these will not be discussed or referenced in this document, either. From this point forward in this document, the term GPDs will refer only to the four chiral-even, twist-2 quark GPDs $H, \tilde{H}, E, \tilde{E}$.

Despite not being sensitive to struck/reabsorbed quark helicity changes, some of the four GPDs of interest for DVCS experiments are sensitive to nucleon helicity changes. The $E$-GPDs are, and the $H$-GPDs are not. Tilded GPDs are sensitive to the struck quark helicity (and are called "polarized" GPDs), while the un-tilded GPDs do not, and are called "unpolarized". Table 1.1 shows the various experimental combinations that allow for access to these GPDs through DVCS on the proton. GPDs listed in red indicate that these are kinematically suppressed, and conditions for DVCS on the neutron are not listed.

\begin{tabular}{|c|c|c|}
\hline \multicolumn{3}{|c|}{ Accessing GPDs through DVCS } \\
\hline Proton Polarization & Polarized Beam & Unpolarized Beam \\
\hline Transverse & & $H, E$ \\
Longitudinal & & $H, \tilde{H}$ \\
Unpolarized & $H, \tilde{H}, E$ & \\
\hline
\end{tabular}

Table 1.1: A table describing how to access GPDs through DVCS on the proton. A GPD listed in red would be kinematically suppressed in such an experiment.

The end goal of accurately modeling GPDs over all kinematic regimes demands a great experimental effort. The measurable cross sections for reactions such as DVCS and DVMP (deeply virtual meson production) can be used to extract GPDs for many combinations of $t, x$, and $Q^{2}$.

For reference, the E12-06-114 experiment, whose results are discussed in this document, used a polarized beam and an unpolarized proton target, meaning the kinematically dominant GPD we access is $H$. 


\subsubsection{Accessing GPDs via DVCS and Factorization}

Understanding GPDs is an important step towards understanding the strong force interaction in kinematic regimes where perturbative QCD cannot be applied. At short distances, and high energy scales, the strong coupling constant $\alpha_{s}<<1$ and the processes are called "hard". However, as we move to lower energies, and distances close to the size of a proton, $\alpha_{s}$ becomes large (>1) and the process is called "soft", because higher order perturbations are no longer suppressed. We rely on a principle called factorization to separate these two parts of the DVCS reaction. If factorization is valid, GPDs extracted are universal for the proton. This means that regardless of experimental setup, the GPDs measured for the proton will be the same.

At leading twist, factorization has been proven to hold for the DVCS reaction [21] [22]. Figure 1.6 depicts the factorization of the hard and soft parts at leading twist, in what is called a "handbag" diagram. In the limits where the handbag diagram approximates the DVCS reaction $\left(Q^{2}>>M^{2}, \frac{|t|}{Q^{2}}<<1\right.$, and $W$ large for fixed $\left.x_{B}\right)$, GPDs at leading twist can be indirectly accessed from experimental observables (for example the cross section and its asymmetries) via helicity-CFF measurements.

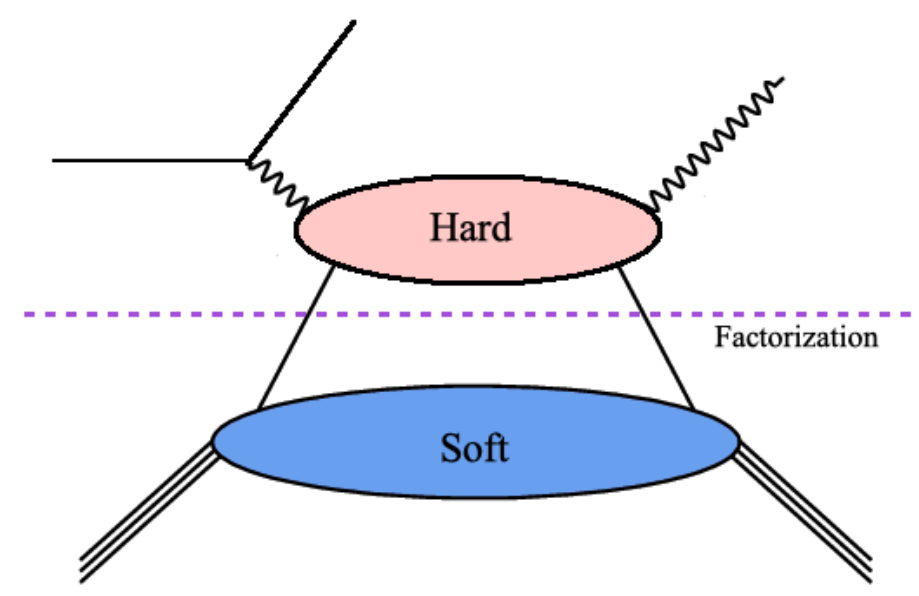

Figure 1.6: The DVCS handbag diagram.

The validity of the handbag diagram, and thus confirmation of the leading twist approximation, can be tested using DVCS observables. A goal of the E12-06-114 experiment is to determine whether or not twist-2 contributions dominate the DVCS amplitude, and the relevant experimental results can be found in section 6.4 .

\subsubsection{Properties of GPDs}

Before discussing how GPDs are experimentally accessed, it is first useful to consider some important properties of GPDs that can help in understanding their theoretical usefulness, and help in measuring them experimentally. 
In general, GPDs depend on $Q^{2}, x, t$, and $\xi$. As was the case with PDFs, a scaling relationship in $Q^{2}$ holds for twist-2 and twist-3 GPDs, and therefore they only depend on $x, t$, and $\xi$ [16]. The dependence of GPDs on many variables means that they contain much important information regarding the structure of nucleons, as compared to the lower dimensional FFs ( $Q^{2}$ dependence only) and PDFs ( $x$ dependence only). This is referred to as the "curse of dimensionality" [9], as GPDs are very difficult to extract. Important properties exist that help overcome the curse of dimensionality, to extract the GPDs experimentally.

The time reversal property of the discussed $\operatorname{GPDs}(H, \tilde{H}, E, \tilde{E})$ is:

$$
H(x,-\xi, t)=H(x, \xi, t)
$$

This property reduces the effective range of $\xi$ from $-1<\xi<1$ to $0<\xi<1$. Two kinematic regimes can be formed by comparing $x$ and $\xi$, namely:

- DGLAP (Dokshitzer, Gribov, Lipatov, Altarelli, and Parisi) region: In this region, $|x|>\xi$. When $x<-\xi$, the nucleon emits an antiquark that is then reabsorbed. Similarly, when $x>\xi$, a quark is emitted and reabsorbed by the nucleon. In this region, GPDs describe the probability to find a quark that carries nucleon (longitudinal) momentum fraction $x+\xi$, that recombines with momentum fraction $x-\xi$. GPDs in this region evolve like the PDFs, according to the DGLAP equations.

- ERBL (Efremov, Radyushkin, Brodsky, Lepage) region: In this region, $-\xi<x<\xi$. Here, GPDs behave like a distribution amplitude, and represent the probability of finding a quark-antiquark pair emitted from the nucleon.

The following relationships exist that reduce GPDs down to FFs and PDFs.

- Making use of the Optical Theorem, we recover PDFs in the forward limit of $t=0$ and $\xi=0$ :

$$
\begin{aligned}
& H(x, 0,0)=\left\{\begin{array}{lc}
q(x), & x>0 \\
-\bar{q}(-x), & x<0
\end{array}\right. \\
& \tilde{H}(x, 0,0)= \begin{cases}\Delta q(x), & x>0 \\
\Delta-\bar{q}(-x), & x<0\end{cases}
\end{aligned}
$$

where $q(\bar{q})$, and $\Delta q(x)(\Delta \bar{q}(x))$ are the quark (antiquark), unpolarized and polarized $(\Delta)$ PDFs.

- FFs are obtained from the zeroth moment of corresponding GPD by integrating over $x$ (at constant $t$, and $\forall \xi$ )

$$
\int_{-1}^{1} H(x, \xi, t) d x=F_{1}(t)
$$




$$
\begin{aligned}
& \int_{-1}^{1} E(x, \xi, t) d x=F_{2}(t) \\
& \int_{-1}^{1} \tilde{H}(x, \xi, t) d x=G_{A}(t) \\
& \int_{-1}^{1} \tilde{E}(x, \xi, t) d x=G_{P}(t)
\end{aligned}
$$

where $F_{1}$ and $F_{2}$ are the Dirac and Pauli Form Factors (discussed in Section 1.1), and $G_{A}$ and $G_{P}$ are the axial and pseudo-scalar Form Factors (the weak interaction counterparts relating to nucleon spin and pseudo-scalar quark density respectively).

- Ji's sum rule allows us to find the total angular momentum $J$ of a flavor- $f$ quark:

$$
J_{f}=\frac{1}{2} \int_{-1}^{1} x\left[H_{f}(x, \xi, 0)+E_{f}(x, \xi, 0)\right] d x
$$

- The property of polynomiality states that if $n$ is even (odd), then the $n^{\text {th }}$ moment in $x$ of a GPD will be a polynomial in $\xi$ of order $n(n+1)$. This means that for GPD $H$ we have:

$$
\begin{aligned}
& \int_{-1}^{1} x^{n} H(x, \xi, t) d x=a_{0}+a_{2} \xi^{2}+a_{4} \xi^{4}+\ldots+a_{n} \xi^{n} \text { (n even) } \\
& \int_{-1}^{1} x^{n} H(x, \xi, t) d x=a_{0}+a_{2} \xi^{2}+a_{4} \xi^{4}+\ldots+a_{n+1} \xi^{n+1} \text { (n odd) }
\end{aligned}
$$

with the $a_{n}$ depending on $t$.

\subsubsection{DVCS and the Bethe-Heitler process}

The Bethe-Heitler (BH) process (Figure 1.7) is experimentally indistinguishable from DVCS. Both are $e p \rightarrow e^{\prime} p^{\prime} \gamma$ reactions, however unlike DVCS, the BH amplitude can be computed using pure $\mathrm{QED}$. The $\mathrm{BH}$ occurrs when the incoming or outgoing electron radiates a photon, and does not involve a struck quark as in the DVCS. Therefore, when we extract information about GPDs from an experimentally measured $e p \rightarrow e^{\prime} p^{\prime} \gamma$ (electron photoproduction) cross section, we need to consider the DVCS $\left(\left|\mathcal{T}_{D V C S}\right|^{2}\right)$ and Bethe-Heitler $\left(\left|\mathcal{T}_{B H}\right|^{2}\right)$ amplitude contributions, and additionally their interference $\left(\mathcal{I}_{D V C S-B H}\right)$. This cross section is expressed in Equation 1.33 as:

$$
\frac{d^{4} \sigma}{d Q^{2} d x_{B} d t d \phi_{\gamma \gamma}}=\frac{\alpha^{3} x_{B} y^{2}}{8 \pi Q^{4} \sqrt{1+\epsilon^{2}}} \frac{1}{e^{6}}\left[\left|\mathcal{T}_{D V C S}\right|^{2}+\left|\mathcal{T}_{B H}\right|^{2}+\mathcal{I}_{D V C S-B H}\right]
$$

In this expression of the cross section, $y=\frac{p \cdot q}{p \cdot k}=\frac{v}{E}$, and $\epsilon=2 x_{B} \frac{M}{Q}$. The interference term $\mathcal{I}=\mathcal{T}_{D V C S} \mathcal{T}_{B H}^{*}+\mathcal{T}_{D V C S}^{*} \mathcal{T}_{B H}$. 


\subsubsection{Parametrization of the Bethe-Heitler amplitude}

The Bethe-Heitler process is purely QED, and we can parametrize the BH amplitude term using elastic Form Factors (Section 1.1). Using a harmonic series expansion of the amplitude in $\phi$, for an unpolarized target we have:

$$
\left|\mathcal{T}_{B H}\right|^{2}=\frac{e^{6}}{y^{2} x_{B}\left[1+4 x_{B}^{2} M^{2} / Q^{2}\right]^{2} t \mathcal{P}_{1}\left(\phi_{\gamma \gamma}\right) \mathcal{P}_{2}\left(\phi_{\gamma \gamma}\right)}\left[c_{0}^{B H}+\sum_{n=1}^{2} c_{n}^{B H} \cos \left(n \phi_{\gamma \gamma}\right)\right]
$$

where

$$
\begin{gathered}
\mathcal{P}_{1}\left(\phi_{\gamma \gamma}\right)=-\frac{1}{y\left(1+\epsilon^{2}\right)}\left[J+2 K \cos \phi_{\gamma \gamma}\right] \\
\mathcal{P}_{2}\left(\phi_{\gamma \gamma}\right)=1+\frac{t}{Q^{2}}+\frac{1}{y\left(1+\epsilon^{2}\right)}\left[J+2 K \cos \phi_{\gamma \gamma}\right] \\
J=\left(1-y-\frac{y \epsilon^{2}}{2}\right)\left(t+\frac{t}{Q^{2}}\right)-\left(1-x_{B}\right)(2-y) \frac{t}{Q^{2}}
\end{gathered}
$$

The coefficients $c_{0}^{B H}$ and $c_{n}^{B H}$ are well-known functions that can all be expressed in terms of the Pauli and Dirac form factors $F_{1}$ and $F_{2}$. Therefore, this term of the cross section does not give us insight into GPDs.
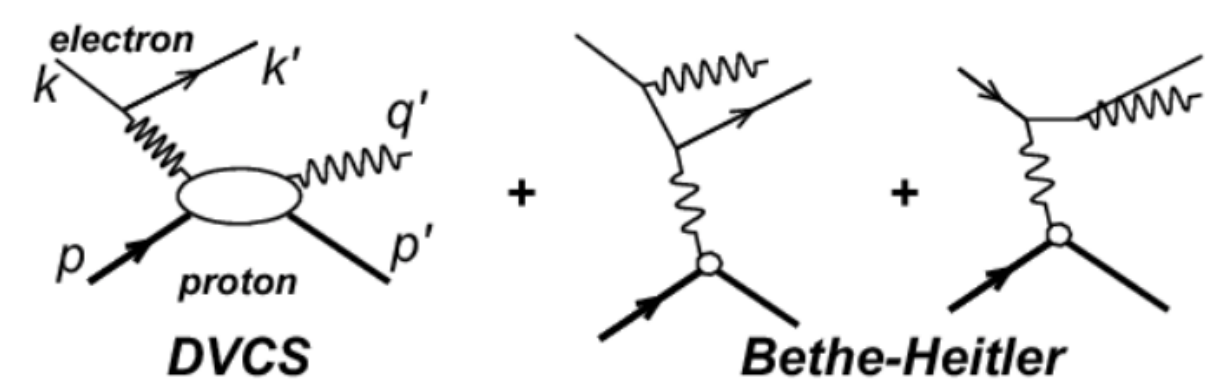

Figure 1.7: DVCS vs. the Bethe-Heitler process. Note that in the Bethe-Heitler, the final state photon arises from Bremsstrahlung radiation of either the incoming or outgoing electron, and not a struck quark.

\subsubsection{CFFs and GPDs}

Unlike the Bethe-Heitler term, the remaining DVCS amplitude and the interference terms $d o$ contain useful information regarding GPDs. Unfortunately this information is not directly accessible in the way Form Factors parameterize the BH term. Instead, we gain insight into GPDs indirectly through their relationship with Compton Form Factors (CFFs), which do directly parameterize the remaining terms, in a very similar way to FFs in the BH component. 
The kinematic variables $\xi$ and $t$ are both measurable experimentally, however, $x$ is not directly measurable in the DVCS reaction. Because of this, GPDs, which depend on all three variables, are not directly accessible from the DVCS cross section. We instead can measure CFFs, which are integrals of combinations of GPDs over $x$, and therefore only depend on the measurable $\xi$ and $t$.

There are four relevant twist-2 $\operatorname{CFFs} \mathcal{F} \in(\mathcal{H}, \tilde{\mathcal{H}}, \mathcal{E}, \tilde{\mathcal{E}})$ relating to the twist-2 GPDs, each having a real and imaginary component. Similarly, there are twist-3 CFFs $\mathcal{F}$ eff $\epsilon$ $\left(\mathcal{H}^{e f f}, \tilde{\mathcal{H}}^{e f f}, \mathcal{E}^{e f f}, \tilde{\mathcal{E}}^{e f f}\right)$ relating to twist-3 GPDs, and gluon transversity CFFs $\mathcal{F}_{T} \in$ $\left(\mathcal{H}_{T}, \tilde{\mathcal{H}}_{T}, \mathcal{E}_{T}, \tilde{\mathcal{E}}_{T}\right)$ which relate to the gluon transversity GPDs. Note that in relation to the helicity-CFFs defined in section 1.3.1, we have the following:

$$
\begin{aligned}
& \mathcal{F}_{++} \approx \mathcal{F} \\
& \mathcal{F}_{0+} \approx \frac{\sqrt{2} \tilde{K}}{\sqrt{1+\epsilon^{2}} Q\left(2-x_{B}+\frac{x_{B} t}{Q^{2}}\right)} \mathcal{F}^{e f f} \\
& \mathcal{F}_{-+} \approx \frac{\tilde{K}^{2}}{2 M^{2}\left(2-x_{B}+\frac{x_{B} t}{Q^{2}}\right)^{2}} \mathcal{F}_{T}
\end{aligned}
$$

where $\tilde{K}$ is defined according to:

$$
\tilde{K}=\frac{1}{Q} \sqrt{\left(1-x_{B}\right) x_{B}+\frac{\epsilon^{2}}{4}} \sqrt{\left(t_{\min }-t\right)\left(t-t_{\max }\right)}
$$

with

$$
\begin{aligned}
& t_{\text {min }}=-Q^{2} \frac{2\left(1-x_{B}\right)\left(1-\sqrt{1+\epsilon^{2}}\right)+\epsilon^{2}}{4 x_{B}\left(1-x_{B}\right)+\epsilon^{2}} \\
& t_{\text {max }}=-Q^{2} \frac{2\left(1-x_{B}\right)\left(1+\sqrt{1+\epsilon^{2}}\right)+\epsilon^{2}}{4 x_{B}\left(1-x_{B}\right)+\epsilon^{2}}
\end{aligned}
$$

To reiterate, in the kinematic regime relevant to this thesis, the contributions from the twist-3 quark and gluon transversity GPDs (and thus CFFs) are expected to be small. The relationship between these GPDs and corresponding CFFs can be found in [56].

The twist-2 quark CFFs are formed from combinations of the GPDs $(H, \tilde{H}, E, \tilde{E})$. The following equations define these CFFs:

$$
\begin{aligned}
& \operatorname{Re} \mathcal{H}(\xi, t)=\mathcal{P} \int_{0}^{1}[H(x, \xi, t)-H(-x, \xi, t)] C^{+}(x, \xi) d x \\
& \operatorname{Re} \mathcal{E}(\xi, t)=\mathcal{P} \int_{0}^{1}[E(x, \xi, t)-E(-x, \xi, t)] C^{+}(x, \xi) d x
\end{aligned}
$$




$$
\begin{aligned}
\operatorname{Re} \tilde{\mathcal{H}}(\xi, t) & =\mathcal{P} \int_{0}^{1}[\tilde{H}(x, \xi, t)-\tilde{H}(-x, \xi, t)] C^{-}(x, \xi) d x \\
\operatorname{Re} \tilde{\mathcal{E}}(\xi, t) & =\mathcal{P} \int_{0}^{1}[\tilde{E}(x, \xi, t)-\tilde{E}(-x, \xi, t)] C^{-}(x, \xi) d x \\
\operatorname{Im} \mathcal{H}(\xi, t) & =-\pi[H(\xi, \xi, t)-H(-\xi, \xi, t)] \\
\operatorname{Im} \mathcal{E}(\xi, t) & =-\pi[E(\xi, \xi, t)-E(-\xi, \xi, t)] \\
\operatorname{Im} \tilde{\mathcal{H}}(\xi, t) & =-\pi[\tilde{H}(\xi, \xi, t)-\tilde{H}(-\xi, \xi, t)] \\
\operatorname{Im} \tilde{\mathcal{E}}(\xi, t) & =-\pi[\tilde{E}(\xi, \xi, t)-\tilde{E}(-\xi, \xi, t)]
\end{aligned}
$$

where $\mathcal{P}$ is the principal part of the integral, and $C^{ \pm}$is:

$$
C^{ \pm}(x, \xi)=\frac{1}{x-\xi} \pm \frac{1}{x+\xi}
$$

CFFs are important to consider because the DVCS cross section actually contains integrals of GPDs over $x$, allowing us to easily express the cross section in terms of combinations of CFFs, and access them directly experimentally. GPDs appear in the cross section as integrals of the form:

$$
\int_{-1}^{1} \frac{H(x, \xi, t)}{x-\xi+i \epsilon} d x=\mathcal{P} \int_{-1}^{1} \frac{H(x, \xi, t)}{x-\xi} d x-i \pi H(\xi, \xi, t)
$$

which mimic the form of the CFFs' real and imaginary parts when the limits of integration are reduced from $[-1,1]$ to $[0,1]$. The parametrization of the DVCS cross section by CFFCs will be discussed more in the following sections.

\subsubsection{Parametrization of the DVCS amplitude}

The squared DVCS amplitude in Equation 1.33 can be expressed as a harmonic series expansion in $\phi_{\gamma \gamma}$ given by:

$$
\left|\mathcal{T}_{D V C S}\right|^{2}=\frac{e^{6}}{y^{2} Q^{2}}\left[c_{0}^{D V C S}+\sum_{n=1}^{n=2}\left(c_{n}^{D V C S} \cos \left(n \phi_{\gamma \gamma}\right)+\lambda s_{n}^{D V C S} \sin \left(n \phi_{\gamma \gamma}\right)\right)\right]
$$

Here $\lambda$ represents the electron beam helicity, and the coefficients $c_{i}^{D V C S}$ and $s_{i}^{D V C S}$ are given respectively in real and imaginary bilinear CFFCs 2 ,

\footnotetext{
${ }^{2}$ The coefficients $c_{n}$ and $s_{n}$ are discussed more in Appendix A
} 


\subsubsection{Parametrization of the DVCS-BH interference term}

The DVCS squared amplitude term only allows us to extract information about the modulus of the CFFs, instead of their real and imaginary components individually. The DVCS-BH interference term of the cross section is important for affording us access to this information. If we again express this term in a harmonic series expansion, we have:

$$
\mathcal{I}=\frac{ \pm e^{6}}{y^{3} x_{B} t \mathcal{P}_{1}\left(\phi_{\gamma \gamma}\right) \mathcal{P}_{2}\left(\phi_{\gamma \gamma}\right)}\left[c_{0}^{I}+\sum_{n=1}^{3} c_{n}^{I} \cos \left(n \phi_{\gamma \gamma}\right)+\lambda s_{n}^{I} \sin \left(n \phi_{\gamma \gamma}\right)\right]
$$

where the coefficients $c_{n}^{I}$ and $s_{n}^{I}$ contain respectively real and imaginary parts of the linear CFFCs 3 ,

\subsubsection{Accessing GPDs in the E12-06-114 Experiment}

In the E12-06-114 experiment, we used an unpolarized target and a polarized beam. In this case, the method used to extract CFFCs, and separate their real Re $\mathrm{CFFC}_{\mathrm{C}}$ and imaginary $\mathrm{Im}_{\mathrm{CFFC}}$ parts, considers the sum and difference of the measured helicity-dependent $e p \rightarrow e^{\prime} p^{\prime} \gamma$ cross sections $\overrightarrow{d \sigma}$ and $\overleftarrow{d \sigma}$. As a reminder, the CFFC notation refers to helicity-dependent $\mathrm{CFF}$ combinations. In the case of the unpolarized cross section, only the $c_{n}$ coefficients contribute, whereas in the polarized case, only the $s_{n}$ coefficients will contribute.

$$
\begin{gathered}
\frac{1}{2}\left(\overrightarrow{d^{4} \sigma}+\overleftrightarrow{d^{4} \sigma}\right) \propto \frac{1}{2}\left(d^{4} \sigma_{\text {unpol }}\right)=\left|\mathcal{T}_{B H}\right|^{2}+\operatorname{Re}_{\mathrm{CFF}}\left[\left|\mathcal{T}_{D V C S}\right|^{2}\right]+\operatorname{Re}_{\mathrm{CFF}}[\mathcal{I}] \\
\frac{1}{2}\left(\overrightarrow{d^{4} \sigma}-\overleftarrow{d^{4} \sigma}\right)=\frac{1}{2}\left(\Delta^{4} \sigma_{\text {pol }}\right) \propto \operatorname{Im}_{\mathrm{CFF}}\left[\left|\mathcal{T}_{D V C S}\right|^{2}\right]+\operatorname{Im}_{\mathrm{CFF}}[\mathcal{I}]
\end{gathered}
$$

Equation 1.57 represents the unpolarized cross section, and equation 1.58 is the polarized one. These cross sections give access to $\operatorname{Re}[\mathcal{H}](\operatorname{Re}[\mathcal{E}])$ and $\operatorname{Im}[\mathcal{H}](\operatorname{Im}[\mathcal{E}])$ when DVCS is performed on the proton (neutron).

\subsubsection{Other access to GPDs}

Extracting CFFs and GPDs from the polarized and unpolarized $e p \rightarrow e^{\prime} p^{\prime} \gamma$ cross sections (Section 1.3.12 is not the only means to study GPDs (although it is the only method utilized in the analysis documented in this thesis). The measurement of a DVMP (Deeply Virtual Meson Production) cross section, such as $e p \rightarrow e^{\prime} p^{\prime} \pi_{0}$, also gives access to GPDs.

Another very important method of extracting CFFs is to measure various cross section asymmetries on proton and neutron targets.

- The beam-charge asymmetry $A_{C}$

... gives access to $\operatorname{Re}[\mathcal{H}, \tilde{\mathcal{H}}, \mathcal{E}]$ and $\operatorname{Im}[\mathcal{H}, \tilde{\mathcal{H}}, \mathcal{E}]$.

\footnotetext{
${ }^{3}$ The coefficients $c_{n}$ and $s_{n}$ are discussed more in Appendix A
} 
- The beam-spin asymmetry $A_{L U}$ (longitudinally polarized beam, unpolarized target) ... gives access to $\operatorname{Im}[\mathcal{H}, \tilde{\mathcal{H}}, \mathcal{E}]$.

- The longitudinal target-spin asymmetry $A_{U L}$ (unpolarized beam, longitudinally polarized target)

... gives access to $\operatorname{Im}[\mathcal{H}]$.

- The transverse target-spin asymmetry $A_{U T}$ (unpolarized beam, transversely polarized target)

... gives access to $\operatorname{Im}[\mathcal{E}, \tilde{\mathcal{E}}, \mathcal{H}, \tilde{\mathcal{H}}]$ with no kinematic suppression of $\mathcal{E}$.

- The double-spin asymmetries $A_{L L}$ and $A_{L T}$ (both beam and target polarized) ... give access to $\operatorname{Re}[\mathcal{H}, \tilde{\mathcal{H}}]$ and $\operatorname{Re}[\mathcal{H}, \tilde{\mathcal{H}}, \mathcal{E}, \tilde{\mathcal{E}}]$ respectively.

A detailed description of the different asymmetries can be found in [?]. Many of the available worldwide GPD data has resulted from these asymmetry measurements. While asymmetry results have less experimental error, the extraction and physical interpretation of the CFFCs is not straightforward.

\subsection{Worldwide experimental status}

A dedicated worldwide effort exists to measure DVCS observables. Although DVCS is the simplest reaction that can be studied to access GPDs, extracting this information is still extremely difficult, mostly because the curse of dimensionality requires these experiments to be performed with many different beam energies, detector configurations, etc., to cover a large phase space in $x_{B}, Q^{2}$, and $t$ over which we hope to model GPDs from data. Figure 1.8 depicts the kinematic regimes of the current experimental efforts to measure DVCS observables. 


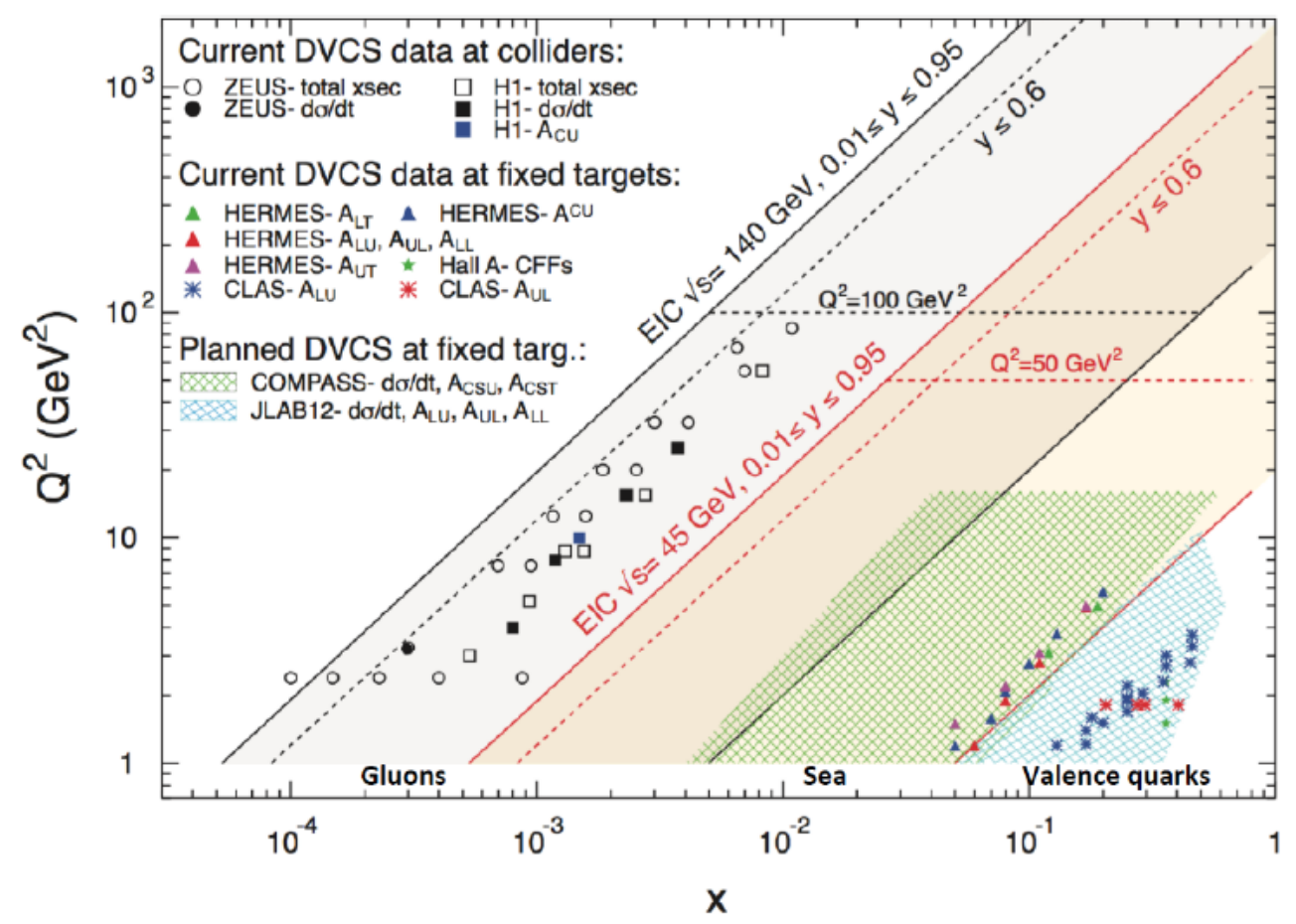

Figure 1.8: The kinematic regimes of worldwide DVCS effort. Figure from [46]. The grey and tan shaded regions show the $x, Q^{2}$ reach of an electron-ion collider for different center-of-mass energies.

This long-term program to extract GPD data points at many different kinematic settings requires a large experimental effort. The majority of this effort exists across three facilities: at DESY's HERA (Hadron Elektron Ring Anlage) accelerator, at CERN's LHC (Large Hadron Collider), and at JLab's CEBAF.

\subsubsection{DESY Experiments}

DESY is a national research center dedicated to the investigation of the structure of matter through particle accelerator experiments. While DESY runs a multitude of accelerator facilities, the relevant DVCS experiments took place at the HERA (Hadron Elektron Ring Anlage) facility.

\subsubsection{H1 and ZEUS}

The H1 and ZEUS detectors were used to study an electron/positron beam collided with a proton beam. These experiments used the beam charge asymmetry method (Section 1.3.13) in the $10^{-4}<x_{B}<10^{-2}$ region of gluon and sea quark dominance. Additionally, these experiments measured total DVCS cross sections, and were able to eliminate Bethe-Heitler contributions with very high beam energies. The relevant references for the H1 and ZEUS DVCS experiments can be found in [25] [26] [27] [28] [29] and [30] 


\subsubsection{HERMES}

The HERMES experiment was a fixed target experiment. Both the target (proton) and beam (electron and positron) could be polarized, allowing the collaboration to perform a nearly complete set of asymmetry measurements to access CFFs $\left(A_{C}, A_{L T}, A_{L U}, A_{U T}\right)$. This experiment covered the $0.04<x_{B}<0.2$ kinematic range. The relevant references for the HERMES DVCS experiments can be found in [31] [32] 33] [34] 35] [36] [37] and [38].

\subsubsection{CERN Experiment}

The COMPASS experiment at CERN was an SPS experiment taking place from 2016-2017 [39]. This experiment took data in the $10^{-3}<x_{B}<10^{-2}$ region, which connects the fixed target and collider experiment regimes. The collaboration measured charge and helicitydependent cross sections, and preliminary results have been recently presented [40].

\subsubsection{JLab Experiments}

Jefferson Lab in Newport News, Virginia, USA houses CEBAF (Continuous Electron Beam Accelerator Facility), and four experimental halls (A, B, C, D).

\subsubsection{CLAS}

Hall B at JLab houses the CLAS (CEBAF Large Acceptance Spectrometer) detector, that spans a large acceptance and solid angle at the expense of having lower luminosity. The E01113 experiment in 2005 measured unpolarized and helicity dependent DVCS cross sections, and the beam-spin asymmetry in the $0.1<x_{B}<0.6$ region. The results of this placed important constraints on $H_{q}$, using a polarized beam and unpolarized hydrogen target.

Later in 2009 the E05-114 experiment measured beam-spin, target-spin, and double-spin asymmetries for a polarized beam on a longitudinally polarized $\mathrm{NH}_{3}$ target. The relevant references for the CLAS DVCS experiments can be found in [41] [42] [43] [44] and [45].

\subsubsection{Hall A}

The Hall A experiments at JLab are high precision, high luminosity, but at the expense of small acceptance. The E00-110 experiment ran in 2004, and measured unpolarized and helicity-dependent DVCS cross sections, using a polarized beam and unpolarized $\mathrm{LH}_{2}$ target. This experiment was important because its fixed $x_{B}=0.36$ data (Figure 1.9p provided a scaling test and demonstrated factorization and leading twist dominance (the validity of the handbag diagram) held in a lower $Q^{2}$ region $\left(1.5 \mathrm{GeV}^{2}<Q^{2}<2.3 \mathrm{GeV}^{2}\right)$. 


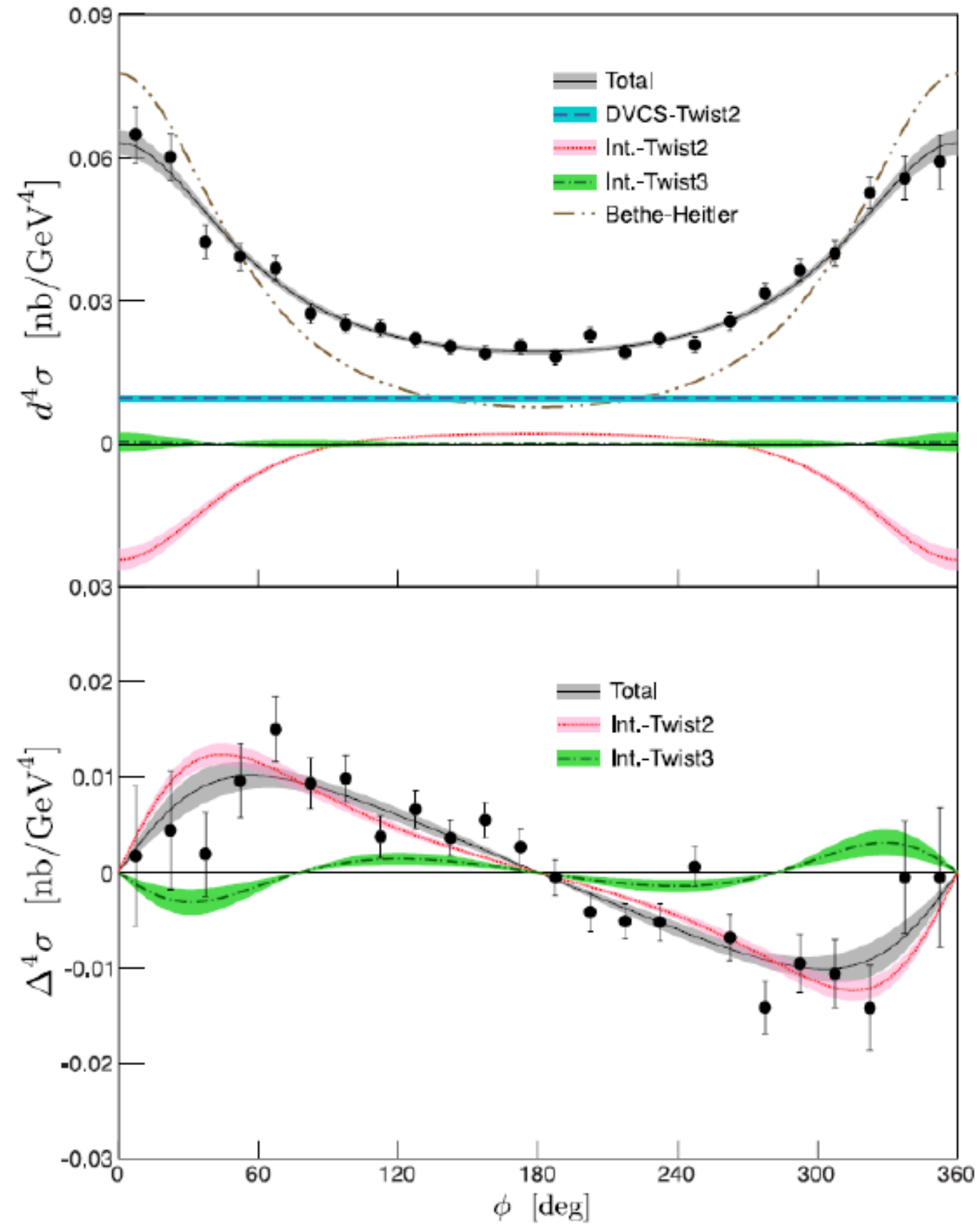

Figure 1.9: The results of the E00-110 experiment [49], showing unpolarized (top) and helicity-dependent (bottom) cross section measurements for $x_{B}=0.36, Q^{2}=2.3 \mathrm{GeV}^{2}$, and $-t=0.32 \mathrm{GeV}^{2}$. The colored bands are fitted combinations of Compton Form Factors that parameterize the experimentally extracted cross section. The DVCS and Int. terms appearing in the legend refer to which term of the cross section the CFF combination was extracted from, either the DVCS amplitude, or the interference term. The twist-3 contribution in each case is small. 
Also in 2004, the E03-106 experiment studied DVCS on the neutron, using a liquid deuterium target, to study $E_{q}$. In 2010, another experiment E08-025 performed DVCS on the neutron, and the E07-007 experiment performed DVCS on the proton.

\subsubsection{Fits to CFF $\mathcal{H}$}

As part of the dedicated GPD effort, one group is attempting to fit the $\mathrm{CFF} \mathcal{H}$ [47].

\subsection{The E12-06-114 DVCS Experiment}

The results of the E12-06-114 experiment are the subject of this thesis. The experiment ran in 2014 and 2016 as a high-energy extension of the previous Hall A experiments. Similarly, a polarized electron beam and unpolarized $\mathrm{LH}_{2}$ target were used to extract absolute polarized and unpolarized DVCS cross sections in a range of $\left(x_{B}, Q^{2}, t\right)$. Figure 1.10 summarizes the kinematic regimes covered by E12-06-114, the first experiment to study GPDs in these regions. Because the experiment was performed in Hall A, the high luminosity affords the capability to measure DVCS cross sections with very high precision.

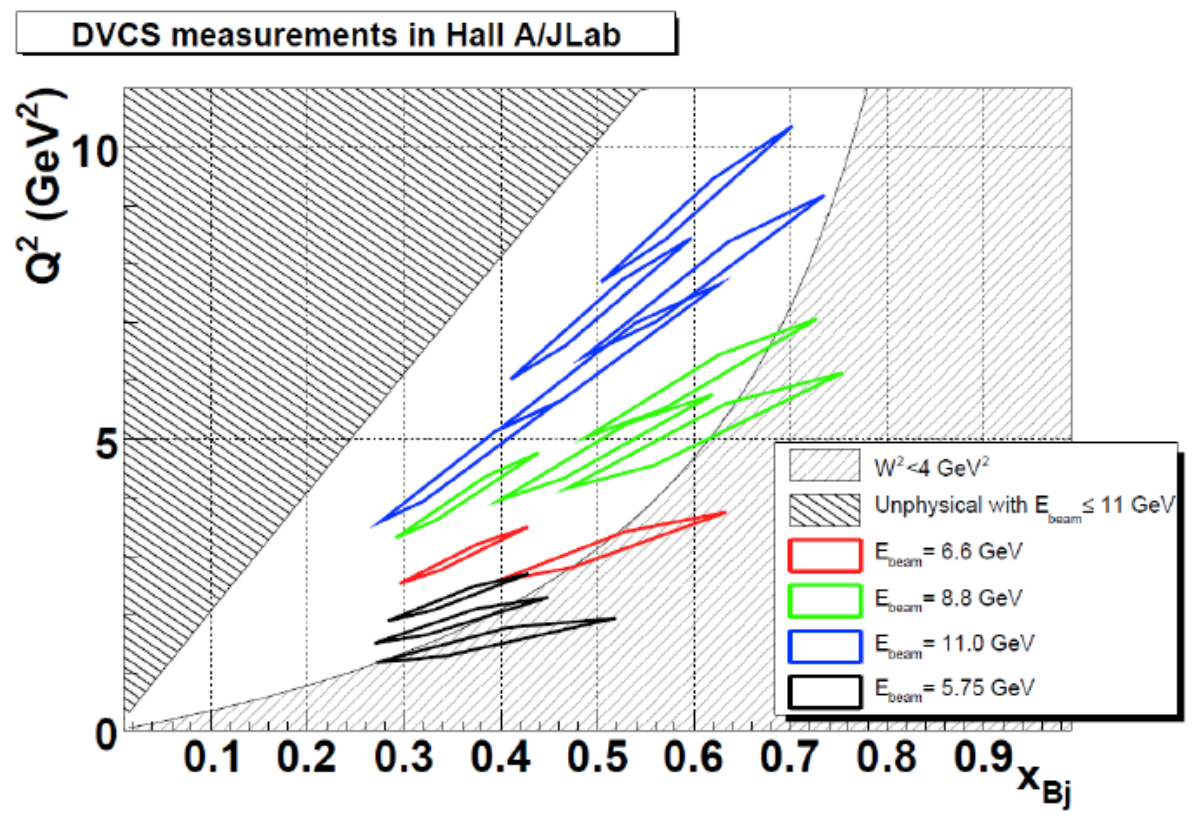

Figure 1.10: The kinematic region spanned by the proposed E12-06-114 DVCS experiment. Note that the exact beam energy values used in the experiment vary from those outlined in this figure, extracted from the proposal [48].

Again like its 2004 and 2010 counterparts, the E12-06-114 experiment will perform a scaling test, now at larger $Q^{2}$, and investigate leading twist dominance. Additionally, we 
will separate the real and imaginary CFFCs contributions to the DVCS amplitude in this leading twist approximation.

Table 1.2 shows the kinematics which had been planned for the E12-06-114 experiment. In this document, the DVCS results are only given for the 481, 482, 483, and 484 settings. The results for the remainder of the kinematics can be found in the thesis of Frederic Georges 63. or Hashir Rashad (thesis pending).

\begin{tabular}{|c|c|c|c|c|c|}
\hline Setting & $x_{B}$ & $Q^{2}\left(\mathrm{GeV}^{2}\right)$ & $\begin{array}{c}E_{\text {beam }} \\
(\mathrm{GeV})\end{array}$ & $t_{\text {min }}\left(\mathrm{GeV}^{2}\right)$ & $t_{\text {max }}\left(\mathrm{GeV}^{2}\right)$ \\
\hline 361 & 0.36 & 3.200 & 6.663 & -0.163 & -0.69 \\
362 & 0.36 & 3.600 & 8.517 & -0.165 & -0.54 \\
363 & 0.36 & 4.470 & 10.617 & -0.167 & -0.54 \\
\hline 481 & 0.48 & 2.700 & 4.483 & -0.321 & -0.58 \\
482 & 0.48 & 4.365 & 8.843 & -0.344 & -0.72 \\
483 & 0.48 & 5.334 & 8.843 & -0.351 & -0.71 \\
484 & 0.48 & 6.900 & 11.023 & -0.359 & -0.96 \\
\hline 601 & 0.60 & 5.541 & 8.517 & -0.661 & -1.47 \\
602 & 0.60 & 6.100 & 8.517 & -0.671 & -1.24 \\
603 & 0.60 & 8.400 & 10.617 & -0.700 & -1.41 \\
604 & 0.60 & 9.000 & 10.617 & -0.706 & -1.28 \\
\hline
\end{tabular}

Table 1.2: Summary of the 11 planned kinematic settings for E12-06-114. The three fixed values of $x_{B}$ with varying $Q^{2}$ allow us to separate the real and imaginary contributions to the DVCS amplitude.

\subsection{Future Experiments}

The study of the many GPDs is a large undertaking, and requires a vast effort to study the entire set in all kinematic ranges. Because of JLab's recent beam energy upgrade, many past experiments can be re-performed with higher $Q^{2}$. For example, the planned CLAS12 suite of experiments are the extension to the CLAS experiments performed in Hall B. Included in this, E12-06-119 [51] covers similar kinematic regimes as E12-06-114, making the former a large acceptance, low luminosity companion to the latter. Another companion experiment, C12-15-004 [53], if approved will complement neutron data from E05-114. Finally, E12-11003 [52] will provide larger kinematic coverage of neutron data.

In Hall C, the planned E12-13-010 experiment [50] will make polarized and unpolarized DVCS cross section measurements on the proton, just as E12-06-114 has done. However, the Hall $\mathrm{C}$ instrumentation allows for an extended kinematic range (up to a $Q^{2}$ of $10 \mathrm{GeV}^{2}$ ) compared to Hall A. Data from the same kinematic regimes taken in E12-06-114 can now be measured at different beam energies, and the extension to new regimes will provide more insight on the validity of leading twist dominance at these settings. 
The future Electron Ion Collider (EIC) will afford a great opportunity for many nuclear particle physics experiments. The collider will feature electron and proton/ion collisions for center of mass energy range of $20-140 \mathrm{GeV}$. The transition regime between sea and valence quarks will be accessible, and a transversely polarized proton beam will make the study of GPD E possible. The EIC will be constructed at Brookhaven National Lab. 


\section{Chapter 2}

\section{Jefferson Lab and Hall A Instrumentation}

Jefferson Lab, in Newport News, Virginia, was founded in 1984 with the mission to explore the atomic nucleus and its constituents. The lab's continuous electron beam accelerator facility (CEBAF, figure 2.1) provided beam to up to three original experimental halls, with a construction started started for a fourth in 2009. The E12-06-114 DVCS experiment was performed in Hall A, which is dedicated to high luminosity, high precision scattering experiments. While the DVCS cross section has been measured at Jefferson Lab previously, a recent $12 \mathrm{GeV}$ upgrade to the accelerator afforded the study of kinematic regions at larger $x_{b}$ and $Q^{2}$ than previously possible. 


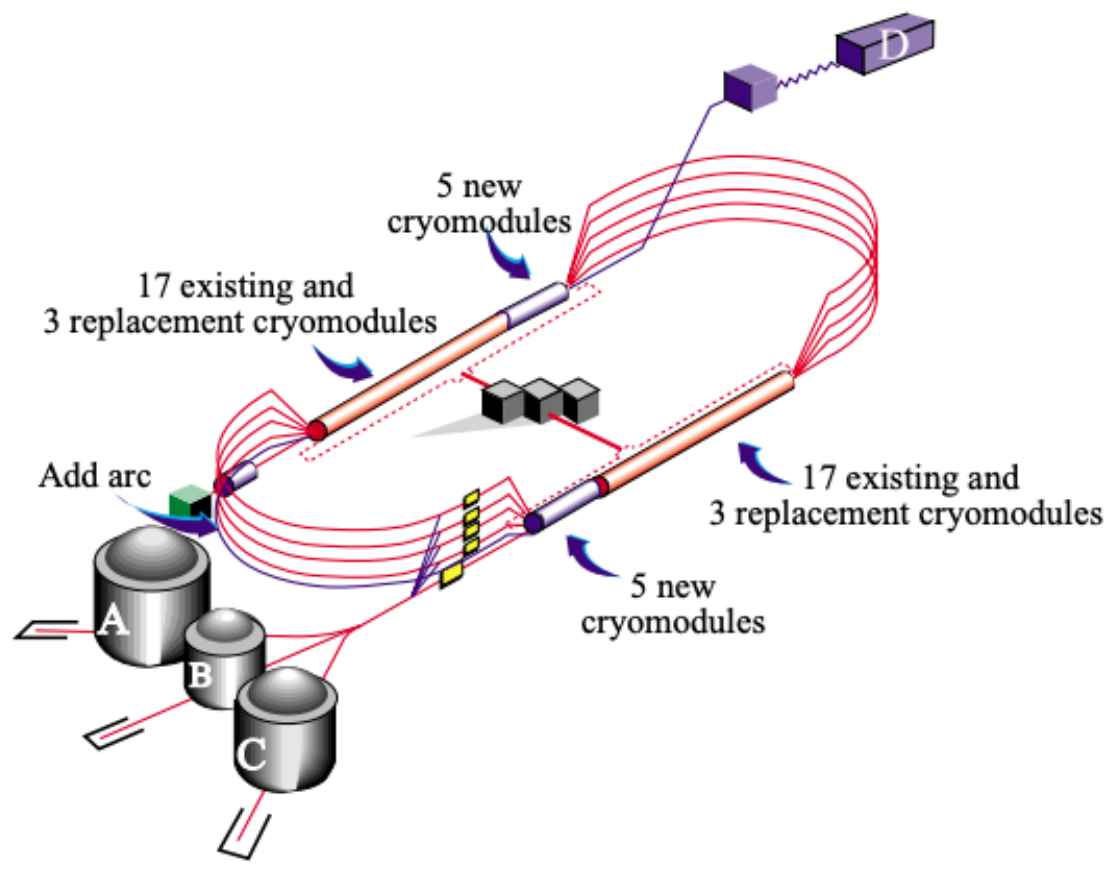

Figure 2.1: A figure depicting the electron accelerator facility and its upgrade at Jefferson lab. Figure from [54]

This chapter details the relevant instrumentation housed at Jefferson Lab [55]. The experimental setup and detector efficiencies will be discussed later in Chapter 3 .

\subsection{CEBAF}

The continuous electron beam accelerator facility (CEBAF) both produces electrons (at the injector) and accelerates them to the experimental halls.

\subsubsection{Injector}

Polarized electrons that reach the halls originate at the injector, where a laser $(780 \mathrm{~nm}, 1497$ $\mathrm{MHz}$ gain-switched) illuminates a photocathode (lattice-strained gallium arsenide (GaAs) crystal) in vacuum to strip electrons. Through optical pumping, electrons in the valence band of the crystal are pushed into the conduction band, with polarization determined by the illuminating laser. A layer of cesium fluoride (CsF) on the crystal surface lowers the potential barrier and increases the likelihood of the polarized electrons to escape the conduction band and enter the vacuum. These electrons are then accelerated by the linacs to the halls. More information about the CEBAF injector can be accessed through the groups wiki page: https://wiki.jlab.org/ciswiki/index.php/Main_Page. 


\subsubsection{Accelerator and $12 \mathrm{GeV}$ Upgrade}

In 2009, plans began to upgrade the electron beam energy capabilities from $6 \mathrm{GeV}$ to 12 $\mathrm{GeV}$. An additional 5 cryomodules were built and installed per linac (previously 20), and an additional recirculating magnet arc was added.

Once polarized electrons are produced at the injector, they enter the accelerator. The beam track is made up of two linacs, north and south, connected by recirculating magnets that bend electrons in an arc connecting the linacs. The two linacs, composed of 25 cryomodules each, that accelerate an electron by $1.1 \mathrm{GeV}$ for each pass. This results in a maximum beam energy reaching $12 \mathrm{GeV}$ after five and a half passes to hall $\mathrm{D}$, and $11 \mathrm{GeV}$ after five passes to halls A, B, and C. The beam current to each individual hall is controlled with RF separators.

\subsection{Hall A Instrumentation}

Hall A is one of four experimental halls at Jefferson Lab. It is dedicated to running high luminosity, high precision scattering experiments. The E12-06-114 experiment used many of the resident Hall A detectors and instrumentation (shown in a view-from-above schematic in Figure 2.2, with the addition of a photon calorimeter used to detect the scattered photon in the DVCS reaction (discussed in Section 3.2.

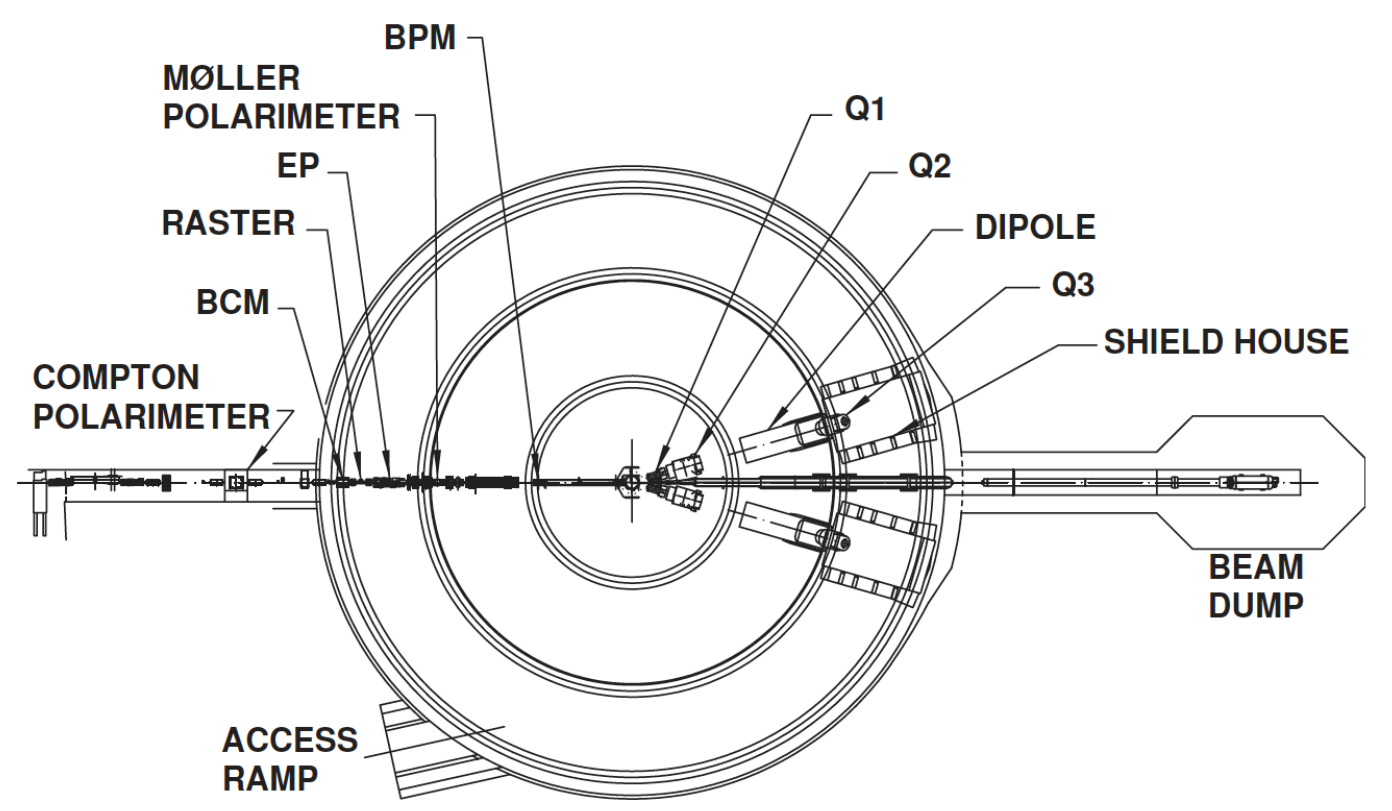

Figure 2.2: A schematic view of beamline components in Hall A.

Extensive details about the Hall A detectors discussed in the sections below can be found 
in 55 .

\subsubsection{Beamline}

The beamline in Hall A has specialized detectors to monitor beam polarization, position, and current.

\subsubsection{Polarimeters}

There are two types of polarimeters in Hall A that monitor the beam polarization, called the Moller and the Compton, that measure the beam polarization through their respective scattering reactions. Beam polarization measurements are necessary for extracting polarized DVCS cross sections.

\subsection{Moller Polarimeter}

The first, the Moller Polarimeter (Figure 2.3), cannot be run simultaneously with production running, and requires dedicated time. The Moller utilizes a supermendur (a combination of Cobalt, Iron, and Vandium) target, polarized using Helmholtz coils, with a QQQD magnet array that bends scattered beam and recoil target electrons to lead glass shower detectors. The asymmetry for beam electrons scattered off the polarized target electrons can be measured to find the overall beam polarization with estimated 1-2\% uncertainty.
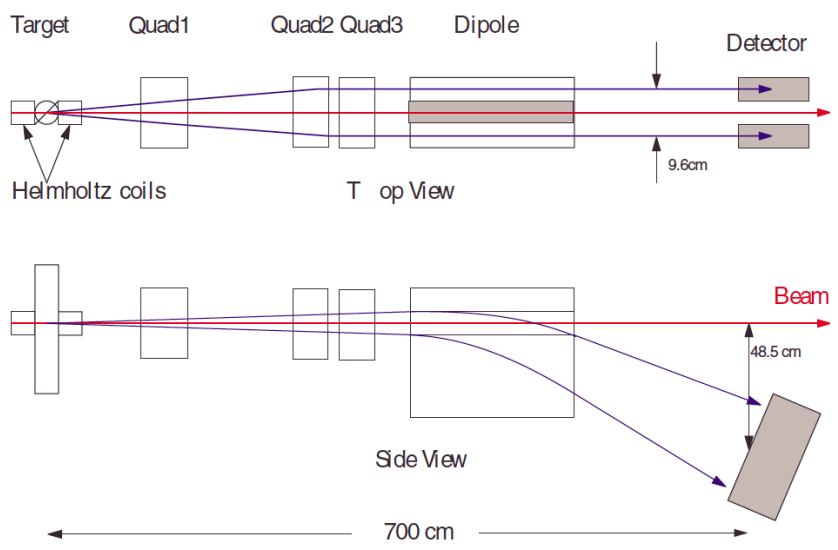

Figure 2.3: A figure depicting the Moller Polarimeter in Hall A at Jefferson Lab.

\subsection{Compton Polarimeter}

The second, the Compton Polarimeter (Figure 2.4), can run simultaneously with production running. The Compton chicane sits in the tunnel upstream of the hall and Moller polarimeter. It uses a $250 \mathrm{~mW}, 1064 \mathrm{~nm}$, laser intensified in a Fabry-Perot cavity, placed in the beamline to scatter polarized photons off beam electrons. The recoil photon is detected in a $\mathrm{PbWO}_{4}$ 
shower detector downstream. The asymmetry of spin aligned vs. spin anti-aligned scattering events is measured to give the overall polarization of the beam with an uncertainty of $1 \%$. Because it is a parasitic detector, the Compton is a good monitor of beam polarization fluctuations over the course of a run period.

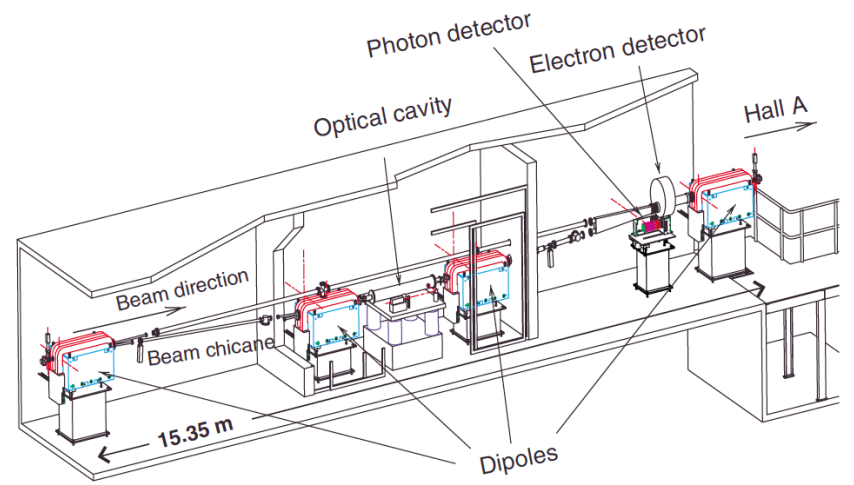

Figure 2.4: A figure depicting the Compton Polarimeter in Hall A at Jefferson Lab.

\subsubsection{Beam Energy Monitors}

The beam energy can be measured in two independent ways, both of which are invasive. The first method is the Arc method, involving the $34.3^{\circ}$ bend of the electron beam in each of the 8 dipoles in either arc of the accelerator. When the beam is tuned to dispersive mode, the momentum of the beam is found using the relationship between the magnetic field integral and its bend (with $k$ a known constant), where $\theta$ is the arc section in radians (see section 4.4 of [5.5]):

$$
p=k \frac{\int \vec{B} \cdot \overrightarrow{d l}}{\theta}
$$

Wire scanners measure the actual bend of the beam, and a 9th reference magnet is used to measure the field of each dipole.

The second method for beam energy measurements is called the eP method, but it not operational after the $12 \mathrm{GeV}$ upgrade. A polyethylene $\left(\mathrm{CH}_{2}\right)$ target sits $17 \mathrm{~m}$ upstream of the target, and uses incident electrons in elastic e-P scattering. The scattered electron and recoil proton angles, $\theta_{e}$ and $\theta_{p}$ respectively, are measured with a stand-alone detector system, and the electron beam energy is found according to

$$
E \approx M_{p} \frac{\cos \left(\theta_{e}\right)+\sin \left(\theta_{e}\right) / \tan \left(\theta_{p}\right)-1}{1-\cos \left(\theta_{p}\right)}
$$

where $M_{p}$ is the mass of the proton. 


\subsubsection{Beam Position Monitors}

The position of the beam is monitored with two Beam Position Monitors (BPMs) each consisting of four antennae placed around the beam. When beam is being delivered to the hall, a current is induced in each antenna. Comparing the signals in each of the four antenna allows the beam position to be deduced. The two BPMs are placed at $7.524 \mathrm{~m}$ and $1.286 \mathrm{~m}$ upstream of the target.

\subsubsection{Beam Current Monitors}

The current of the beam is monitored with RF cavities (which varying approximate gains) and an Unser monitor. The Unser is used a reference when comparing signals seen from the RF cavities during BCM calibration, to measure the gains and offsets of each cavity. The RF cavities are stainless steel cylindrical waveguides tuned to the beam frequency $(1.497 \mathrm{GHz})$. As beam is delivered to the hall, it produces a magnetic field that is magnified in each $\mathrm{RF}$ cavitiy. This field induces a voltage output that is proportional to the beam current. The BCM sits $25 \mathrm{~m}$ upstream of the target.

\subsubsection{Target}

The Hall A target chamber contains multiple targets mounted on a ladder, to be moved into the beamline, for different run type purposes. For the E12-06-114 experiment, a $15 \mathrm{~cm}$ long aluminum cylinder (63.5 $\mathrm{mm}$ in diameter, with $4 \mathrm{~mm}$ thick cell walls) filled with $\mathrm{LH}_{2}$ was used for DVCS on the proton. The mounted targets used could have included a $\mathrm{D}_{2}$ target, for DVCS on the neutron for example, but this was not used in the experiment discussed. The liquid hydrogen is circulated and cooled throughout the run, to avoid boiling. Pressure, density, and temperature of the cryotarget are constantly monitored throughout running.

In addition to the $\mathrm{LH}_{2}$ loop, optics targets and empty targets are used for special runs. The optics target used for Spring 2016 running contained 5, 1mm thick carbon foils spaced $3.5 \mathrm{~cm}$ apart. For Fall 2016 running, a similar optics target was used, however with 9 foils instead of 7 . An empty target is in every way the same as the $\mathrm{LH}_{2}$ target, but empty. This can be used to study the effects of the cell walls.

\subsubsection{High Resolution Spectrometers}

Hall A at Jefferson Lab houses two high resolution spectrometers (HRS), left and right, consisting of two quadrupoles, followed by a dipole, and another quadrupole (QQDQ), and a large detector package. Typically, the left HRS (or arm) is used to detect leptons, and the right arm detects hadrons. These spectrometers are used to find a particle's position and momentum, as it scatters from the target. There is however a trade off between high resolution and large acceptance; while each HRS offers high resolution in these kinematic measurements, the acceptance is rather small (Table 2.1). 


\begin{tabular}{|c|c|c|}
\hline Parameter & Acceptance & Resolution \\
\hline$\theta$ & $\pm 60 \mathrm{mrad}$ & $1 \mathrm{mrad}$ \\
\hline$\phi$ & $\pm 30 \mathrm{mrad}$ & $0.5 \mathrm{mrad}$ \\
\hline$\frac{\delta p}{p}$ & $\pm 4.5 \%$ & $1 \times 10^{-4} \frac{\mathrm{GeV}}{c}$ \\
\hline
\end{tabular}

Table 2.1: Design standards for an HRS

\subsubsection{Design Standards}

Table 2.1 shows acceptance and resolutions for the HRS. From the center of the physical HRS opening, $\phi(\theta)$ represents the horizontal (vertical) angular acceptance of scattered particles from the target. The momentum acceptance parameter is called $\delta p=p-p_{0}$, with $p$ a particles momentum, and $p_{0}$ the central momentum setting which can be set from 0.3 to $4.0 \frac{\mathrm{GeV}}{\mathrm{c}}$. The DVCS experiment used only the left arm, but the concurrently running GMP [59] experiment used the right arm. Figure 2.5 depicts the beamline of Hall A leading to the left and right HRSs.

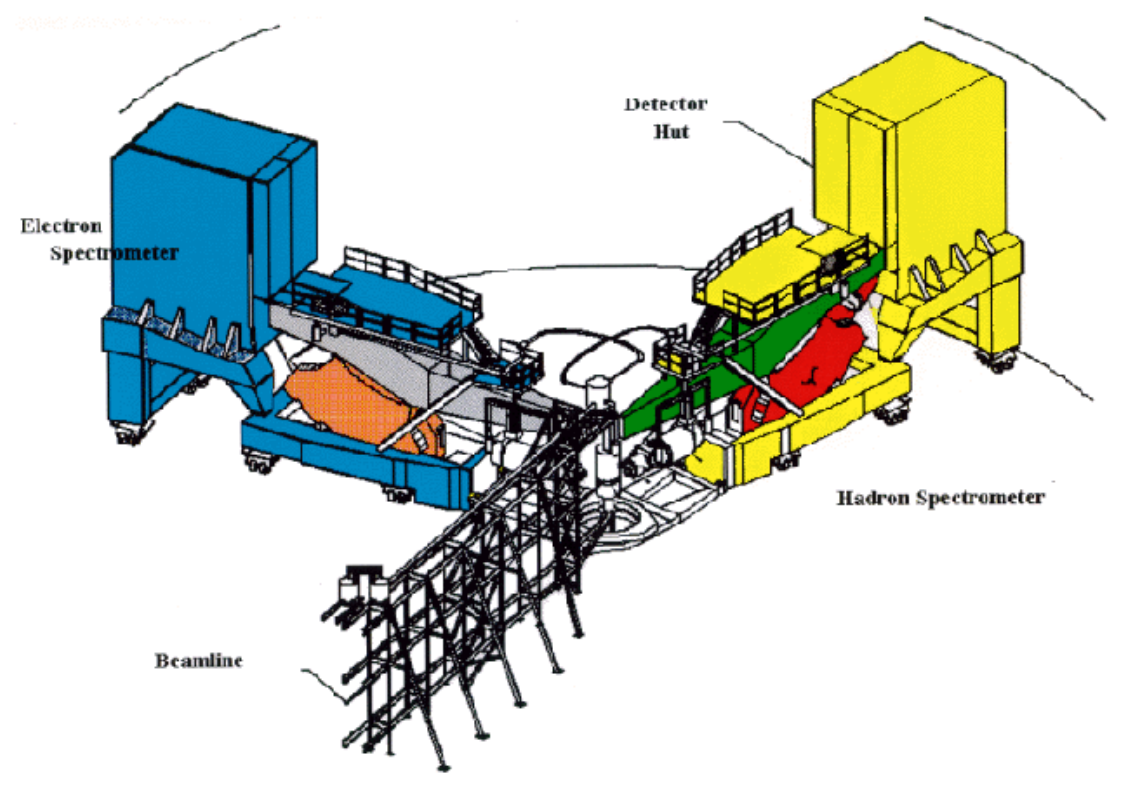

Figure 2.5: A figure depicting the left and right high resolution spectrometers in Hall A at Jefferson Lab.

\subsubsection{Detector package}

Each of the spectrometers is responsible for identifying a scattered particle's type and trajectory, as well as triggering the data acquisition software to record events. Figure 2.6 shows 
the L-HRS detector package. Electrons first travel through two Vertical Drift Chamber (VDC) planes, pass through to S0 and S1 scintillators, through a gas cherenkov, through the S2M scintillator, and then finally an array of electromagnetic shower detectors used to reject pions.

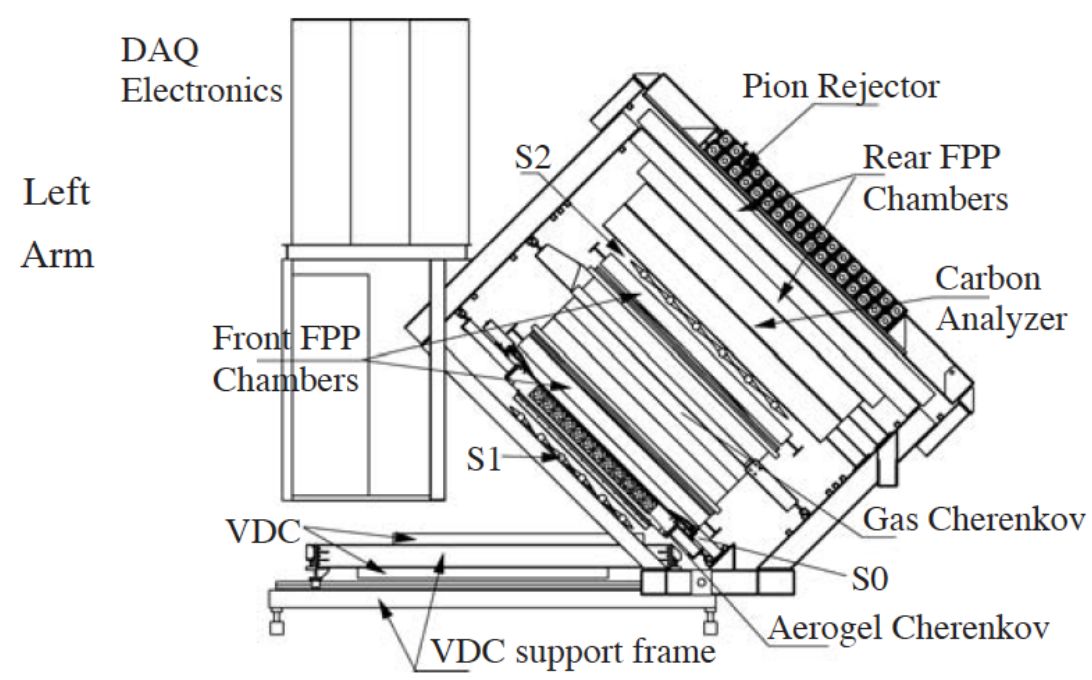

Figure 2.6: A schematic of the detector package for the left HRS. Note that the S2 scintillator plane is referred to in this document as the $\mathrm{S} 2 \mathrm{M}$, the scintillator plane sitting two meters downstream of the S1 plane.

\subsubsection{Particle Identification}

A gas Cherenkov detector, filled with $\mathrm{CO}_{2}$ (index of refraction $n=1.00041$ ) is used to identify electrons. Because of their low mass, electrons with momenta down to $0.017 \frac{\mathrm{GeV}}{c}$ in the detector produce Cherenkov radiation, while pions require a momentum $p_{\pi} \geq 4.8^{c} \frac{\mathrm{GeV}}{c}$. The maximum central momentum $p_{0}$ of the HRS is only $4 \mathrm{GeV}$, implying that the presence of radiation in the gas Cherenkov signals an electron has been detected.

It is possible that a heavier particle can sometimes produce a signal in the Cherenkov due to knock-out electrons. A two-layer array lead glass shower detector (referred to as the pion rejector, Figure 2.7] sits at the back of the detector package. This detector is used to reject heavier particles such as pions that may have triggered a signal in the Cherenkov. The combination of gas Cherenkov and shower detector is used to suppress pion contamination from the leptonic arm of DIS and DVCS data sets. 


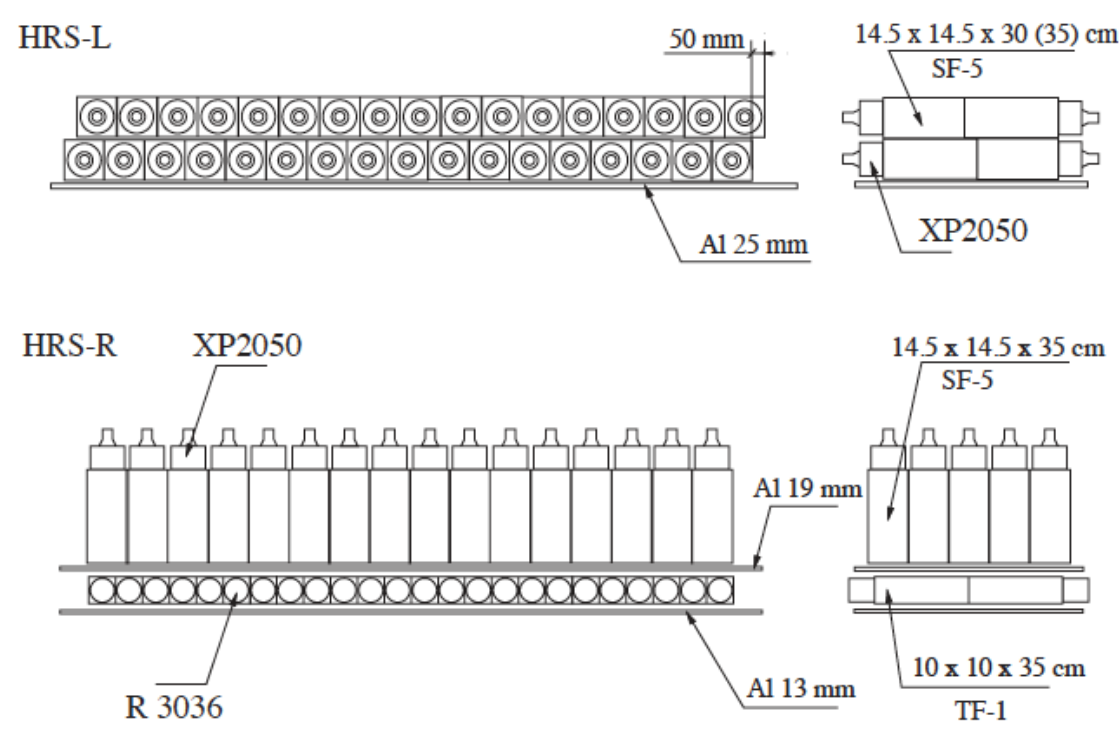

Figure 2.7: A schematic of the pion rejector.

\subsubsection{Particle tracking}

After a scattered particle is transported through the QQDQ magnet array, its trajectory is tracked in vertical drift chambers (VDCs). There are two VDCs, each of which is composed of two planes (oriented at $90^{\circ}$ with respect to each other) of 184 wires arranged array (Figure 2.8). Each plane lies parallel to the floor of the hall, so a particle with $p_{0}$ should intersect the planes at $45^{\circ}$ with respect to the normal of the VDC plane.

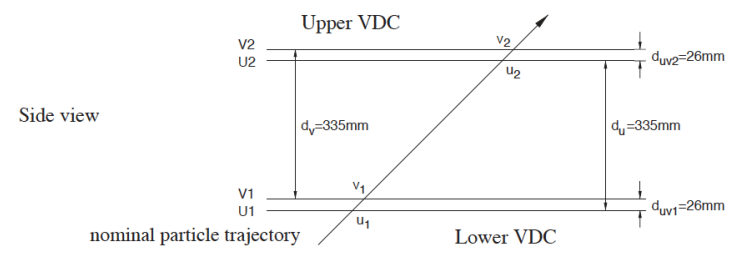

(a) A side view of VDC planes

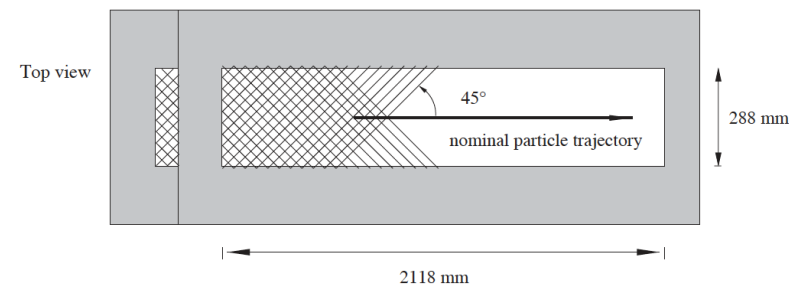

(b) A top view of the VDC planes.

Figure 2.8: Figures showing the side (left) and top (right) views of the vertical drift chambers.

Each chamber is filled with argon and ethane gas in a 50/50 ratio. As particles pass through the drift chambers, the gas is ionized, creating electrons that then "drift" in an electric field, to wires the shortest time of flight distance away (Figure 2.9). This creates a sequence of wire hits for a passing particle. The time of flight associated with each electron wire hit, and the drift velocity, allow for particle path reconstruction. 


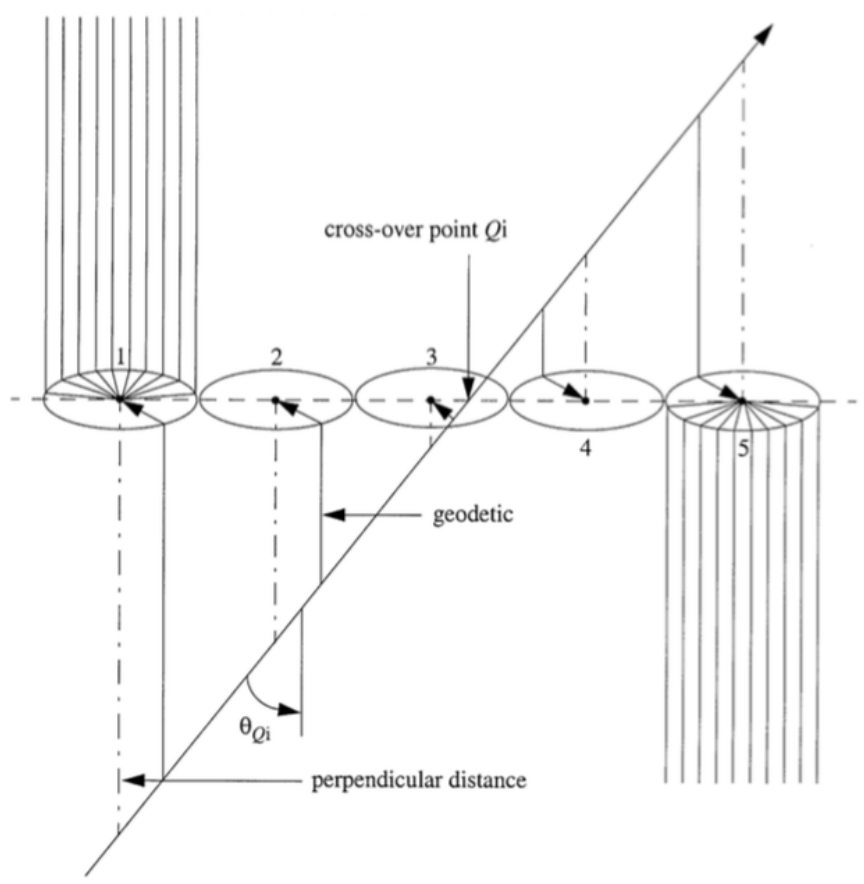

Figure 2.9: A figure depicting the result of a particle passing through the VDC.

\subsubsection{Scintillators}

Three scintillator planes (S0, S1, and S2M) are installed in each HRS to trigger the DAQ readout. Each plane consists of 6 scintillator paddles, each with a PMT on either end. A signal seen in the two PMTs of a paddle signals to the HRS electronics that a particle has passed into the HRS. Both the S0 and S2M planes were used in triggering events used in Spring and Fall 2016 running. Note that S2M and S2 may be used interchangeably when discussing the L-HRS scintillator planes. The "M" after S2 refers to the detector sitting two meters downstream of the S1 plane. This document uses the "M" convention, while other documents may not. 


\section{Chapter 3}

\section{Setup for E12-06-114 Experiment}

The DVCS experimental setup includes the use of standard Hall A equipment, such as the left HRS and the $\mathrm{LH}_{2}$ cryotarget. Both the Moller and Compton polarimeters were used to study the beam polarization for Spring and Fall 2016 running, in order to extract the electron-helicity dependent DVCS cross sections. In addition to these detectors, a $16 \times 13$ element $\mathrm{PbF}_{2}$ calorimeter was placed in the hall to detect the outgoing photon.

\subsection{Kinematic Settings}

The 11 kinematic points to be completed in the E12-06-114 experiment (Table 3.1) were chosen for three values of $x_{B}: 0.36,0.48$, and 0.60 , each with multiple fixed values of $Q^{2}$. The following table lists $x_{B}, Q^{2}, E_{\text {beam }}$ the electron beam energy, $\theta_{H R S}$ the angular position of the HRS, $p_{H R S}$ the central momentum setting of the HRS, and $\theta_{C a l}$ the angular position of the calorimeter in the hall.

Table 3.1: Description of actual Kinematic Settings used for the E12-06-114 experiment.

\begin{tabular}{|c|c|c|c|c|c|c|}
\hline Setting & $x_{B}$ & $Q^{2}\left(\mathrm{GeV}^{2}\right)$ & $E_{\text {beam }}(\mathrm{GeV})$ & $\theta_{H R S}\left({ }^{\circ}\right)$ & $p_{H R S}(\mathrm{GeV})$ & $\theta_{\text {Cal }}\left({ }^{\circ}\right)$ \\
\hline 361 & 0.36 & 3.200 & 7.361 & 22.830 & 2.71 & 10.592 \\
362 & 0.36 & 3.600 & 8.218 & 20.985 & 3.187 & 11.635 \\
363 & 0.36 & 4.470 & 10.619 & 18.675 & 3.998 & 10.618 \\
\hline 481 & 0.48 & 2.700 & 4.477 & 37.138 & 1.485 & 15.198 \\
482 & 0.48 & 4.365 & 8.823 & 20.243 & 3.996 & 15.184 \\
483 & 0.48 & 5.334 & 8.821 & 26.269 & 2.920 & 11.728 \\
484 & 0.48 & 6.900 & 10.972 & 24.923 & 3.360 & 10.069 \\
\hline 601 & 0.60 & 5.541 & 8.518 & 24.564 & 3.594 & 15.892 \\
602 & 0.60 & 6.100 & 8.517 & 27.823 & 3.098 & 14.050 \\
603 & 0.60 & 8.400 & 10.619 & 29.003 & 3.154 & 11.014 \\
604 & 0.60 & 9.000 & 10.617 & 33.039 & 2.621 & 9.633 \\
\hline
\end{tabular}


The experiment was allocated a total of 88 PAC days to be divided among the different kinematic settings as follows in table 3.2. This was the first experiment to run following the $12 \mathrm{GeV}$ upgrade, however, and the accelerator could not always produce reliable beam at the high energies. Overall, only about 50\% of our total statistics were achieved, and two kinematics were completely abandoned.

Table 3.2: PAC allocation of beam time for kinematic settings

\begin{tabular}{|c|c|c|}
\hline Setting & PAC Days & \% Complete \\
\hline 361 & 3 & 100 \\
362 & 2 & 100 \\
363 & 1 & 100 \\
\hline 481 & 5 & 100 \\
482 & 4 & 56.6 \\
483 & 4 & 76.4 \\
484 & 7 & 53.0 \\
601 & 13 & 100 \\
602 & 16 & 0 \\
603 & 13 & 100 \\
604 & 20 & 0 \\
\hline
\end{tabular}

\subsection{Calorimeter}

The outgoing photon in the DVCS reaction (see expected energies in table 5.1 is detected in a $16 \times 13$ array of $\mathrm{PbF}_{2}$ blocks (Figure 3.1) that sit downstream of the target. Each block is wrapped in Tyvek and then Tedlar to help prevent the shower transmission into an adjacent block. Lead fluoride (a Cherenkov medium) was chosen in anticipation of a large hadronic background. Because of the Cherenkov material's short light pulse, pileup effects would be minimal.

Each block of the array is $3 \times 3 \times 18.6 \mathrm{~cm}^{3}$, encompassing 20 radiation lengths, and has optically coupled to the end a fast response PMT. The Molière radius of $\mathrm{PbF}_{2}$ is 2.2 $\mathrm{cm}$, and showers are contained in the incident block plus the 8 surrounding blocks. For a centrally-struck block (as opposed to the edge), 90\% of the total energy is contained in the central block. 


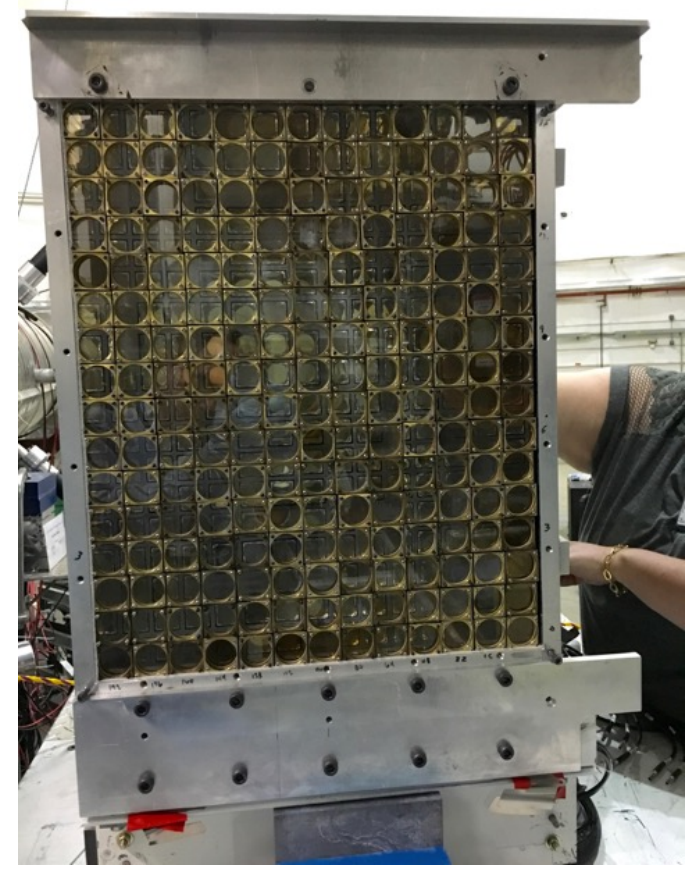

Figure 3.1: A picture showing the photon calorimeter used in the DVCS experiment.

The calorimeter must be regularly calibrated, as the energy resolution of the calorimeter degrades over time due to radiation damage 5.3.3. Several shielding measures were taken to help reduce the back and damage to the calorimeter. First, the calorimeter was installed in a light-tight box. Second, extra beam pipe shielding was added to protect against multiple scattering from the target chamber or beam pipe. Finally, two plastic plates were installed in front of the calorimeter to reduce background from low energy $\gamma \mathrm{s}$.

\subsection{Data Acquisition and Triggers}

Data acquisition (DAQ) in the experimental halls is run using CODA software (CEBAF online data acquisition). Detector signals are sent to analog-to-digital converters (ADCs), time-to-digital convertors (TDCs), and scalers are assembled in VME crates, whose outputs are controlled by the readout controllers (ROCs) located in each crate. Logic combinations can be made of the many ROC signals coming from the many detectors in the hall. These logic signals are then used to form different triggers for recording data. The Trigger Supervisor (TS) is responsible for forming the triggers, and will only accept a new trigger when all ROCs are available.

For S0, each PMT signal is input to a discriminator whose output is fed to a logic module. That module outputs the S0 logic signal whenever a coincidence between the left and right PMTs occurs for one of the 6 paddles in the plane. Similar electronics are used for the $\mathrm{S} 1$ and S2M scintillator planes to produce the S1 and S2M logic signals. Signals from the 
Cherenkov PMTs are summed in groups and the Cherenkov logic signal is generated when any of the summed signals surpasses a preset level, corresponding to the minimum threshold for electrons.

There are a number of different signal combinations that trigger the recording of an event 3.3. The most important triggers are the DIS trigger and the DVCS trigger (level-1 and level-2). The DIS trigger is defined as the coincidence Cer \& (S2M or S0). The level-1 DVCS trigger is defined as a coincidence of the Cer \& S2M.

The level-1 DVCS trigger initiates a readout of each block of the photon calorimeter's flash-ADC (fADC). The signal in each block of the calorimeter is integrated over the 128 ns sampling window of its fADC. These signals are summed for every possible $2 \times 2$ block combination, and compared to a preset threshold. The photon logic signal is generated when the sum is above the set threshold for any block combination. A level-2 DVCS trigger fires when both the level-1 DVCS and photon logic signals are present, and the data from the HRS and calorimeter are recorded by the ROCs by the level- 2 trigger.

Table 3.3: The combination triggers used for data acquisition.

\begin{tabular}{|c|c|}
\hline Trigger & $\begin{array}{l}\text { Event } \\
\text { Type }\end{array}$ \\
\hline $\begin{array}{l}\text { S0 \& } \\
\text { Cer }\end{array}$ & $\begin{array}{c}\text { DIS } \\
\text { (Kin-48 } \\
\text { settings } \\
\text { only) }\end{array}$ \\
\hline $\begin{array}{c}\text { S2M \& } \\
\text { Cer }\end{array}$ & DIS \\
\hline $\begin{array}{c}\text { S2M \& } \\
\text { Cer }\end{array}$ & $\begin{array}{c}\text { DVCS } \\
\text { (level-1) }\end{array}$ \\
\hline S2M \& & DVCS \\
\hline $\begin{array}{c}\text { Cer \& } \\
\text { photon } \\
\text { in } \\
\text { calorime- } \\
\text { ter }\end{array}$ & (level-2) \\
\hline
\end{tabular}

The following figure 3.2 shows an example of the different coincidence triggers and the event types they trigger for Kinematic 481. The coincidence triggers are used to trigger the recording of events, but event recording still includes a record of the signals from individual detectors. Note that the S1 detector was not used during the E12-06-114 experiment.

Different coincidence triggers are assigned prescale values to reduce deadtime effects. Deadtime occurs when a ROC is busy recording data from a detector, and is therefore not available to record new incoming events. Prescaling the S2M \& Cer coincidence trigger by 2, for example, would cause the ROC to only record every other S2M \& Cer event. For high event rates, this technique helps reduce deadtime. 


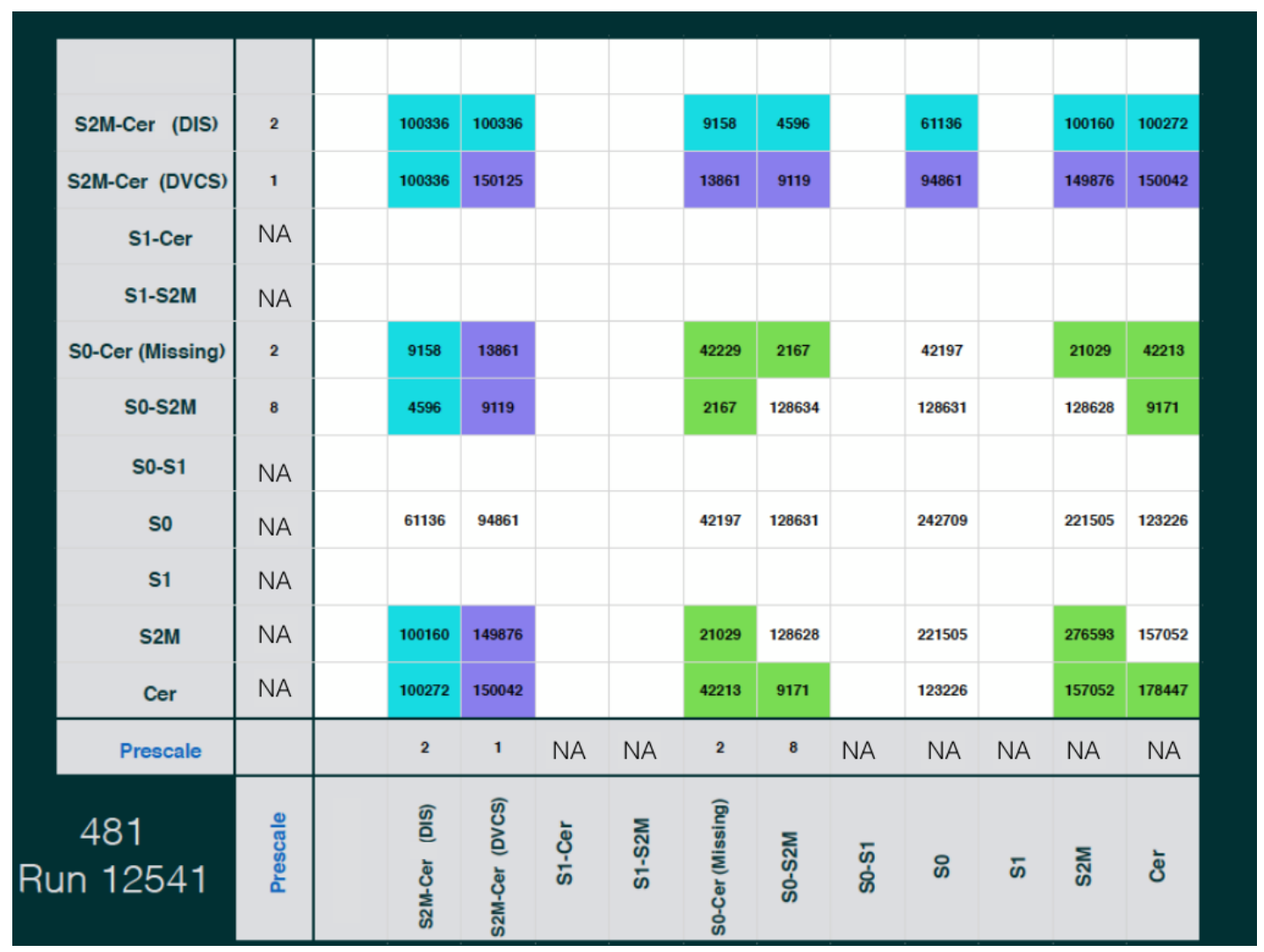

Figure 3.2: An example of the number of events recorded for each trigger combination in approximately one hour of data taking. Note that the S1 was not used during the E12-06114 experiment, so no events appear for all S1 combinations and the prescale is listed as not available (NA). Because single S0, S2M, and Cher signals do not form triggers themselves, the prescale is noted as NA. The "missing" qualifier for the S0 \& Cer DIS combination will be discussed in section 3.3 .1 below.

\subsubsection{S0/S2M Timing and Missing Events}

Using S2M to trigger DIS and DVCS events is merely to signal that a particle is seen in the HRS. It therefore doesn't matter if S0 or S2M is used to signal a DIS or DVCS event. However, it was discovered after data taking was finished, that the presence of an S0 signal interfered with event tagging. Specifically, the S0 signal would sometimes arrive at the electronics before the signal from S2M. These events then get tagged as only S0\&Cer, with no presence of S2M, and do not carry the expected DIS or DVCS event tag. Events that fall into this category are referred to as "Missing" events, as they are missed by the normal event selection cuts for DIS and DVCS, and need to be taken into account during analysis. This correction will be discussed in the next chapter in section 4.1.1.1. 


\subsection{HRS Magnetic Optics}

The L-HRS measures the dynamics of particles scattered from the target. Particle type and trajectory (momentum, position, scattering angle) are measured using the HRS detector package discussed in section 2.2.3.2. However, the detector package measures the particle's coordinates after it has passed through a QQDQ magnet array, instead of directly after scattering. In order to correlate particle coordinates measured in the HRS detector with particle trajectory immediately after scattering, effects of the magnetic optics on a scattered particle trajectory need to be understood.

To do this, the standard transport formalism (for small-acceptance spectrometers) for is used. We define the central momentum of the spectrometer to be the momentum a particle would need to pass through the center of the first and last quadruple, and the center dipole. The trajectory of a particle with central momentum $p_{c}$ is called the central ray. The trajectory of a particle is described using coordinates $\left(x, y, \theta, \phi, \delta_{p}\right)_{z}$, describing the position of the particle at point $z$ in the trajectory relative to the central ray. The coordinates $x$ $(y)$ and $\theta(\phi)$ correspond to the position and angle in the vertical (horizontal) plane, and $\delta$ describes the momentum difference $\frac{p-p_{c}}{p_{c}}$.

A $5 \times 5$ matrix $T$ defines the first order transport of a particle's coordinates between the target scattering vertex and the focal plane of the spectrometer, where the detector package is located (Equation 3.1).

$$
\left[\begin{array}{l}
x \\
y \\
\theta \\
\phi \\
\delta
\end{array}\right]_{f p}=[T]\left[\begin{array}{l}
x \\
y \\
\theta \\
\phi \\
\delta
\end{array}\right]_{t g}
$$

The matrix $T$ represents the first-order magnetic optics of the spectrometer. After $T$ is known for a given spectrometer setting, the detector package is used to measure the transport coordinates at the focal plane, and the coordinates at the target vertex can then be reconstructed. In the case specific to the Hall A L-HRS, the focal plane coordinates are reduced to $x_{f p}, y_{f p}, \theta_{f p}=\frac{d x}{d z} f p$, and $\phi_{f p}=\frac{d y}{d z f p}$, measured by the VDCs, and the target coordinates are reduced to $\delta, y_{t g}, \theta_{t g}$, and $\phi_{t g}$. The coordinate $x_{t g}$ is taken to be zero by requiring the beam position on target be within $250 \mu \mathrm{m}$ of the origin. Equation 3.1 can then be inverted to give the optics matrix described in figure 3.3 .

$$
\left[\begin{array}{l}
\delta \\
\theta \\
y \\
\phi
\end{array}\right]_{t g}=\left[\begin{array}{cccc}
\langle\delta \mid x\rangle & \langle\delta \mid \theta\rangle & 0 & 0 \\
\langle\theta \mid x\rangle & \langle\theta \mid \theta\rangle & 0 & 0 \\
0 & 0 & \langle y \mid y\rangle & \langle y \mid \phi\rangle \\
0 & 0 & \langle\phi \mid y\rangle & \langle\phi \mid \phi\rangle
\end{array}\right]\left[\begin{array}{l}
x \\
\theta \\
y \\
\phi
\end{array}\right]_{f p}
$$

Figure 3.3: The HRS Optics Matrix 

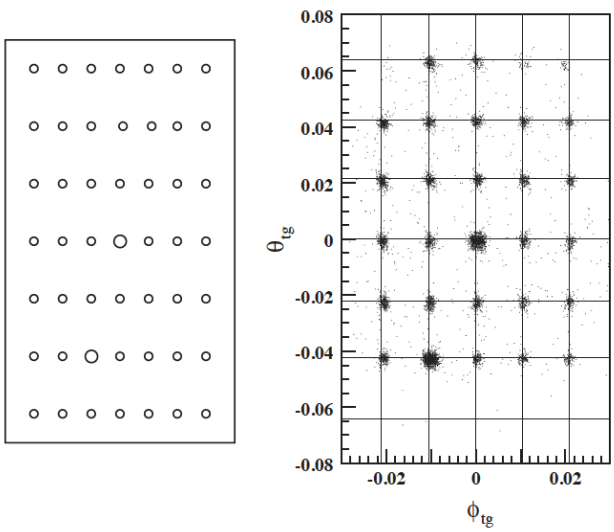

(a) Sieve plate reconstruction

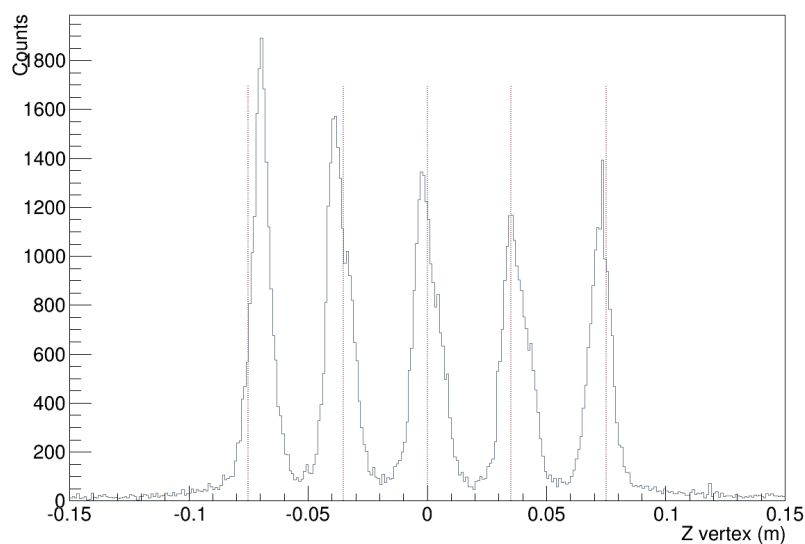

(b) Accumulated data using a multi-foil target. Dotted lines show where the foils are expected to be seen.

Figure 3.4: Figure (a) shows a depiction of the sieve plate used for optics calibration, and a reconstruction of data taken with the sieve in place. Figure (b) shows data reconstructing a multi-foil target.

The method of calibrating magnetic optics involves the use of a special optics target (typically 5 or 9 carbon foils spaced a known distance apart) and a lead sieve plate, placed at the spectrometer entrance. The sieve plate is a plate of lead with an array of holes of varying size to eliminate ambiguity of its orientation. Two runs are taken with the foil target: one with the sieve in place, and the other without. When the sieve is in place, scattered particles are only able to enter the spectrometer through the holes in the sieve. Particle paths are then reconstructed (Figure 3.4 a) (from focal plane coordinates back to target coordinates) to match the hole pattern of the sieve, for each of the carbon foils of the target, in order to optimize particle position and angle in the optics matrix. When the sieve is not used, the foil array should be reconstructed, with foils seen in the correct locations (Figure $3.4 \mathrm{~b}$ ). In general, the optics matrix should not depend on beam energy, particle scattering angle, or central HRS momentum setting, assuming each magnet in the QQDQ array operates at optimal field strength. Unfortunately, the first quadruple, Q1, was not operating correctly for both Spring and Fall running, and special treatment of the optics was required.

\subsubsection{Spring 2016 Optics}

Ideally, the optics matrix is determined for each kinematic setting, to correct for any imperfections in magnet field strength. In Spring 2016 running all four settings (481, 482, 483, and 484) had dedicated optics runs. Having these dedicated runs was especially important in Spring 2016, because it was known that the superconducting Q1 could not achieve high 
enough current for certain kinematic settings, which would affect the magnetic optics differently for each. Table 3.4 below lists how "detuned" the superconducting Q1 was for each Spring 2016 setting. Here detuned refers to what percentage of the ideal current the Q1 could achieve (100\% meaning the magnet could reach full current).

Table 3.4: Q1 status during Spring 2016 Running

\begin{tabular}{|c|c|c|c|c|}
\hline Setting & $\theta_{H R S}$ & $\begin{array}{c}p_{H R S} \\
(\mathrm{GeV})\end{array}$ & Q1 Used & Q1 Detuned \% \\
\hline 481 & $37.1^{\circ}$ & 1.5 & Superconducting & Not Detuned $(100 \%)$ \\
482 & $20.2^{\circ}$ & 4.0 & Superconducting & Detuned $(62 \%)$ \\
483 & $26.3^{\circ}$ & 2.9 & Superconducting & Detuned $(85 \%)$ \\
484 & $24.9^{\circ}$ & 3.4 & Superconducting & Detuned $(74 \%)$ \\
\hline
\end{tabular}

\subsubsection{Fall 2016 Optics}

Due to the problems with Q1 in the Spring, a conventional quadrupole replaced the superconducting Q1 for Fall 2016 running. Because the faulty Q1 was replaced, dedicated optics runs were only taken for 2 out of the 4 kinematic settings that ran during that time (although the optics matrix should be independent of kinematic setting). Two optics runs were taken for large $\left(25^{\circ}\right)$ and small $\left(18^{\circ}\right)$ L-HRS angles, and optics matrix optimization was performed by the GMP collaboration. One single optics matrix was then used for the entirety of Fall 2016 running. Unfortunately, it was discovered after data collection was finished that the new conventional Q1 was saturating (meaning the magnetic field strength was a saturation percentage less than expected for the applied current), giving poor reconstructions of optics target foils, and thus poor reconstruction of production data. Table 3.5 shows the status of Q1 during Fall 2016 running.

Table 3.5: Q1 status during Fall 2016 Running

\begin{tabular}{|c|c|c|c|c|}
\hline Setting & $\theta_{H R S}$ & $\begin{array}{c}p_{H R S} \\
(\mathrm{GeV})\end{array}$ & Q1 Used & Q1 Saturated \\
\hline 362 & $21.0^{\circ}$ & 3.2 & Conventional & Saturated $(0.8 \%)$ \\
363 & $18.7^{\circ}$ & 4.0 & Conventional & Saturated $(6.4 \%)$ \\
601 & $24.6^{\circ}$ & 3.6 & Conventional & Saturated $(3.0 \%)$ \\
603 & $29.0^{\circ}$ & 3.2 & Conventional & Saturated $(0.7 \%)$ \\
\hline
\end{tabular}

Because each kinematic setting sees a different magnet saturation, each setting requires a unique optics matrix. However, optics runs were not performed for each setting, and optics matrix calibration cannot be performed as it was for Spring 2016 running. To get around this set back, the COSY simulation tool was used to simulate transport of target coordinates to focal plane coordinates for a given Q1 saturation. Comparing data and simulation allowed for corrections to be made for each kinematic setting's optics matrix (Figure 3.5). 


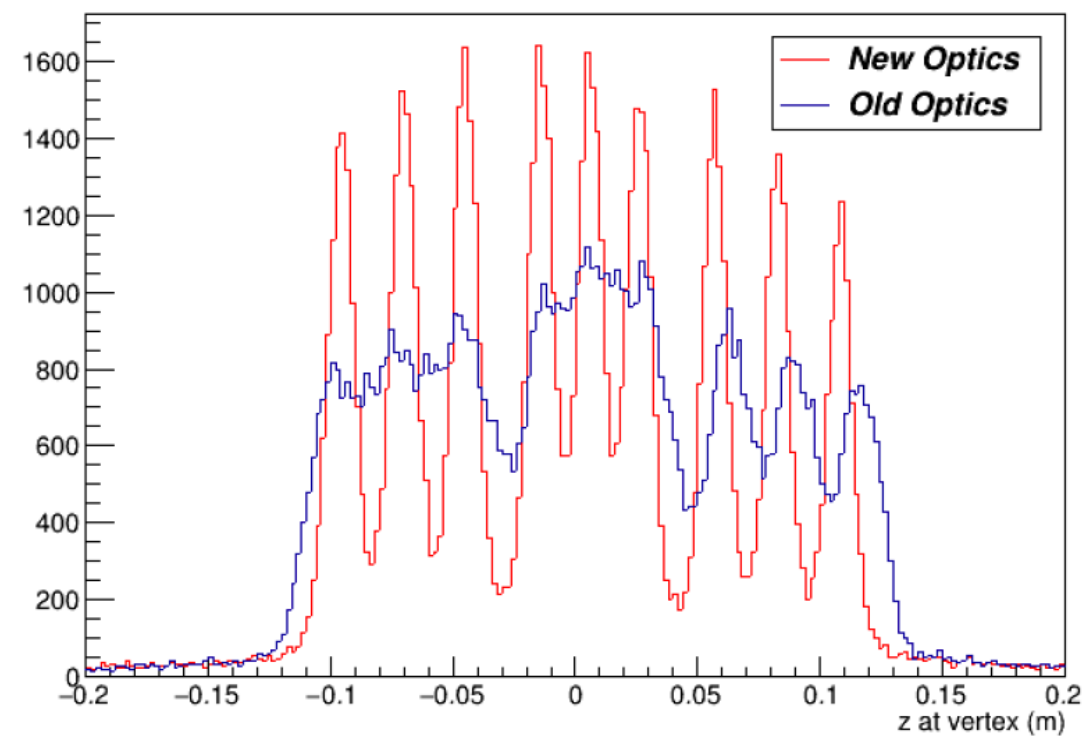

Figure 3.5: Plot from Bishnu Karki showing foil reconstruction before (blue) and after (red) optics matrix corrections for kinematic 363.

\subsubsection{Acceptance and R-Functions}

After an accurate optics matrix has been implemented in the Hall A analyzer software, data from the HRS focal plane can be transported to target coordinates, describing the trajectory and momentum of a particle as it scattered from the target. The originating position within the target, $z_{\text {vertex }}$ (in Hall coordinates [55]), along with the momentum and angles of scattering, completely describe the particle's trajectory before entering the HRS. These, however, are dependent on the optics matrix, and are merely assigned to a trajectory that appeared to have originated at those coordinates. For example, if a scattered particle scrapes along the collimator before entering the HRS, the optics matrix is unable to discern this scenario from one of a particle scattering along the beamline, but in a strange location (i.e. outside the target window). Because of this ambiguity, it may appear that the HRS is receiving events outside of the allowed acceptance region. Thus, cuts are made in the data to include only events from a "good", or well understood, region of acceptance, where the aforementioned events would be eliminated.

\subsubsection{R-Functions}

Cuts must be applied to select a set of events that pass cleanly through the HRS. The acceptance region of each HRS may seem straightforward from a design standard point of view, but making efficient cuts on this acceptance is more complicated than implementing 1 -D cuts on each of the four target variables $\left\{y_{t g}(\mathrm{~m}), \theta_{t g}, \phi_{t g}, \delta p\right\}$. Note that $\delta p$ here is the same as the variable $\delta$ in section 3.4 , and all coordinates are unit-less except for $y_{t g}$ which 
is measured in meters for the R-Function. This is because calculated values of variables such as $\phi_{t g}$ and $y_{t g}$ are correlated, and one variable being outside a proposed 1-D acceptance bound does not necessarily mean that event should be discarded. For instance, if $\phi_{t g}$ was large, meaning the particle scattered at a large vertical angle, it could still be accepted by the HRS, provided $y_{t g}$ was small. Cutting only on a single variable at once would eliminate a large fraction of good events. Figure 3.6 for example shows initial 1D cuts defined for a run with kinematic setting 483. Of an initial 192,242 good electrons, only 62,337 pass the combined cuts, meaning $67.6 \%$ of the data was lost from the acceptance cuts. 

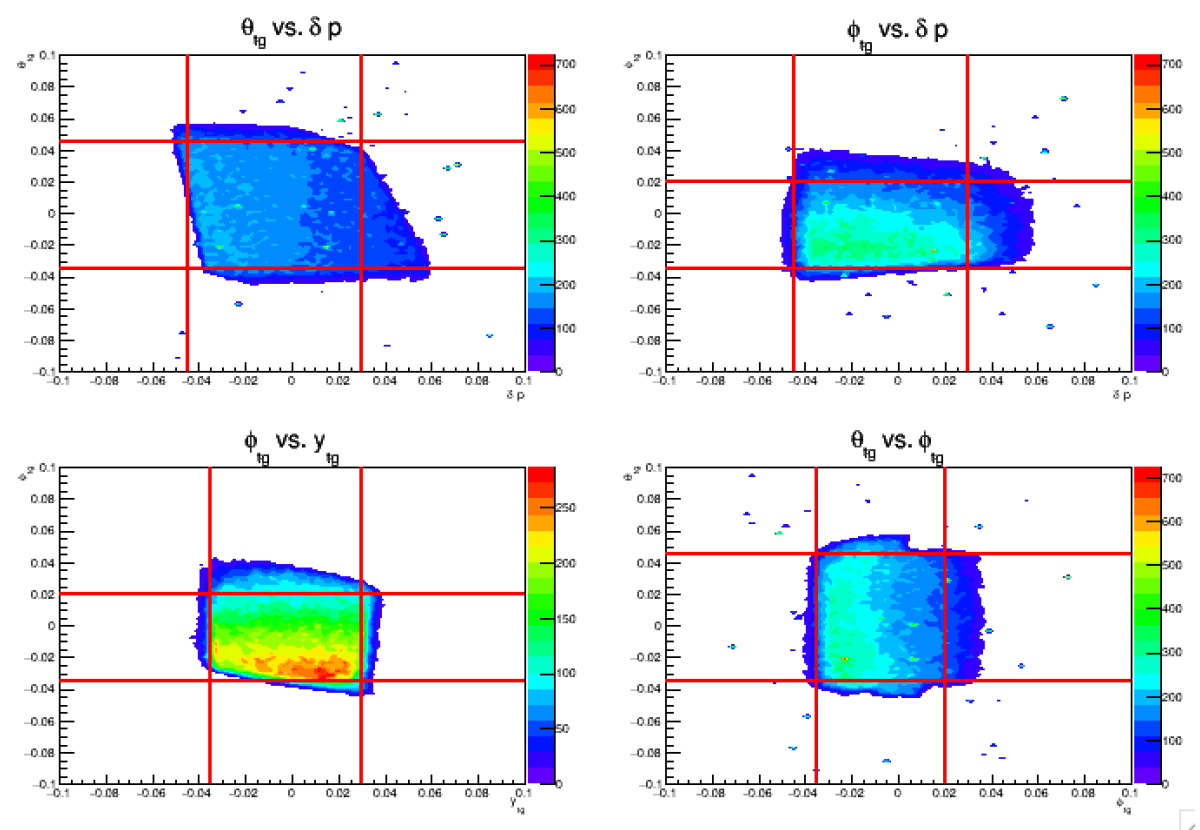

(a) before $1 \mathrm{D}$ cuts on all variables
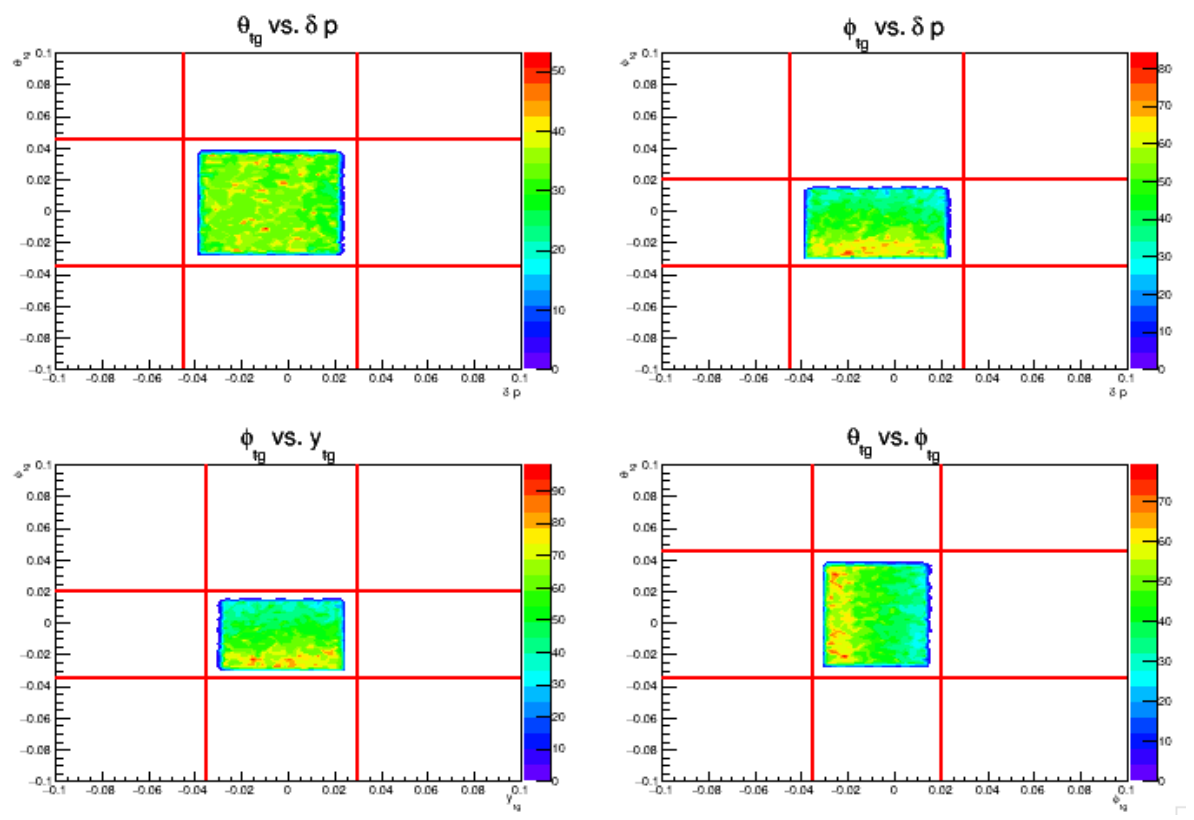

(b) after $1 \mathrm{D}$ cuts on all variables

Figure 3.6: Figures showing HRS data before and after 1D acceptance cuts (red) have been applied to the four target variables $\left\{y_{t g}(\mathrm{~m}), \theta_{t g}, \phi_{t g}, \delta p\right\}$.

Ideally, a 4 dimensional surface would be defined in the volume defined by coordinates $\left\{y_{t g}, \theta_{t g}, \phi_{t g}, \delta p\right\}$, where events inside the surface would pass through the HRS cleanly, and events outside would not. An R-Function defines this 4 dimensional surface, and for every event $i$ having reconstructed target coordinates $\left\{y_{i}, \theta_{i}, \phi_{i}, \delta p_{i}\right\}$, the corresponding R-Value is 
defined as the distance the point lies from the cut boundary according to:

$$
R_{i}= \pm \sqrt{\left(\frac{y_{i}-y_{c u t}}{D_{y}}\right)^{2}+\left(\frac{\theta_{i}-\theta_{c u t}}{D_{\theta}}\right)^{2}+\left(\frac{\phi_{i}-\phi_{c u t}}{D_{\phi}}\right)^{2}+\left(\frac{\delta p_{i}-\delta p_{c u t}}{D_{\delta p}}\right)^{2}}
$$

The parameters $\left\{D_{y}, D_{\theta}, D_{\phi}, D_{\delta p}\right\}$ define the metric and are discussed below. The point $\left\{y_{c u t}, \theta_{\text {cut }}, \phi_{\text {cut }}, \delta p_{\text {cut }}\right\}$ is the point on the cut surface that minimizes the distance $R_{i}$. Positive values of the leading \pm are chosen for points inside the cut surface, and negative values for those outside. With this definition, a single cut can be made on the value $R_{i}$ for every event. The cut on $R_{i}$ can be "loosened" or "tightened" to include more or fewer events from the 4 -D acceptance region.

Unfortunately, we cannot visualize 4-D objects to define this cut, so we instead define a series of $2 \mathrm{D}$ cuts in the planes shown in 3.7 .
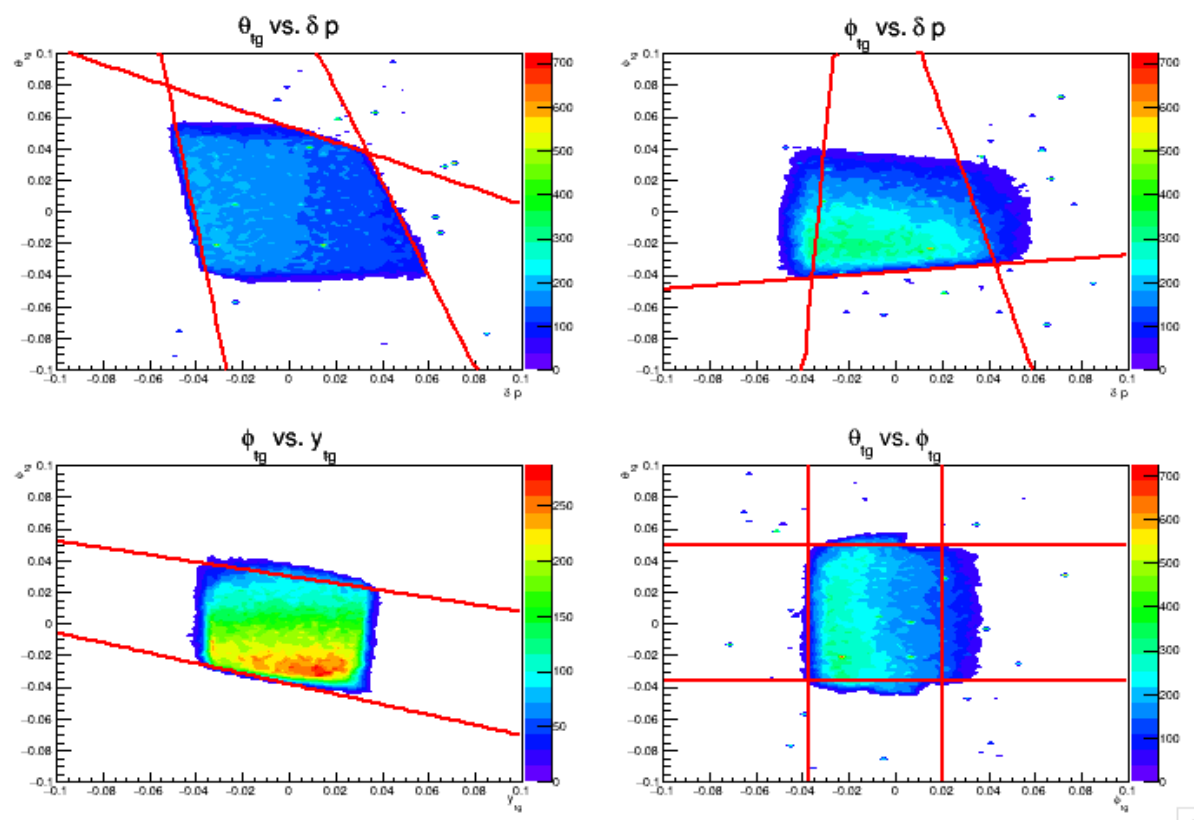

Figure 3.7: A figure showing the four 2D acceptance planes and the 12 cuts used to defined the R-Function for this kinematic setting.

As an example, the cut with the negative y-intercept defined in the $\phi_{t g}$ vs. $y_{t g}$ plane in figure 3.7 has the functional form:

$$
\phi_{t g}=-0.25\left(m^{-1}\right) y_{t g}-0.04
$$

The R-Value associated with this cut would be found using

$$
R_{i}= \pm \sqrt{\left(\frac{y_{i}-y_{c u t}}{D_{y}}\right)^{2}+\left(\frac{\phi_{i}-\phi_{c u t}}{D_{\phi}}\right)^{2}}
$$


Repeating this for all 12 cuts yields 12 values, the least of which is defined as the overall $\mathrm{R}$-Value for the event. If the R-Value is positive, the event lies inside all 12 cuts, and if the $\mathrm{R}$-Value is negative, the event lies outside at least one cut.

The metric parameters $\left\{D_{y}, D_{\theta}, D_{\phi}, D_{\delta p}\right\}$ are chosen as the distance in each variable that it takes for the number of events to drop by $50 \%$ (on the edge of acceptance with the steepest drop-off). Inspecting the planes in figure 3.7, these values are approximately found to be $\left\{D_{y}, D_{\theta}, D_{\phi}, D_{\delta p}\right\}=\{0.006 \mathrm{~m}, 0.005,0.005,0.008\}$. Normalizing the metric parameters to $D_{\theta}$ gives $\{1.2 \mathrm{~m}, 1,1,1.6\}$. However, the region in which this distance is found is poorly defined. Moreover, numerical experimentation found the R-Function procedure to have little sensitivity to the metric selection. To remain consistent with earlier work, assuming that $y_{t g}$ is in meters, the following metric is chosen:

$$
\left\{D_{y}, D_{\theta}, D_{\phi}, D_{\delta p}\right\}=\{1 \mathrm{~m}, 1,1,1\}
$$

During the writing of this thesis, an algebraic error in calculating the distance $R_{i}$ was found that underestimated the actual distance by up to $3 \%$. This is not expected to affect the extracted cross section results, as the process of selecting the cut on $R_{i}$ described in Section 4.1.1.5 should guarantee events passing the cut are in the good acceptance region.

Figure 3.8 shows the same Kin 483 run as figure 3.6 (b.), now with a positive R-Cut applied. Out of again an initial 192,242 good electrons, now 100,566 events pass the acceptance cut, only a $47.7 \%$ loss of statistics. Note that defining four cuts in every plane is not required to "close" the acceptance region (the $\phi, y$ plane for example only has 2 ). The cuts from the other planes would make those additional cuts redundant. 

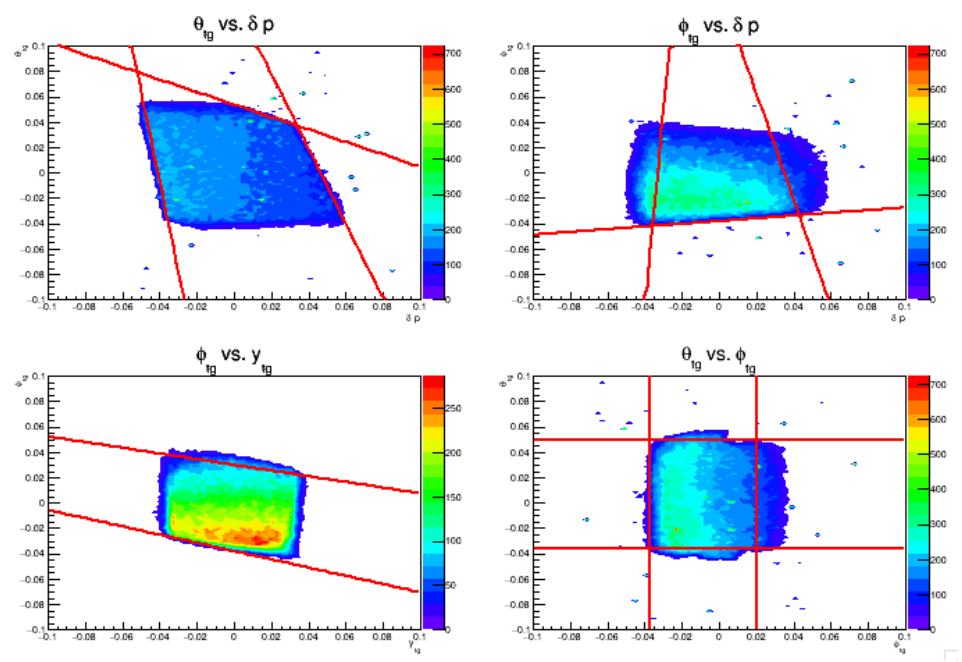

(a) before R-Function acceptance cut
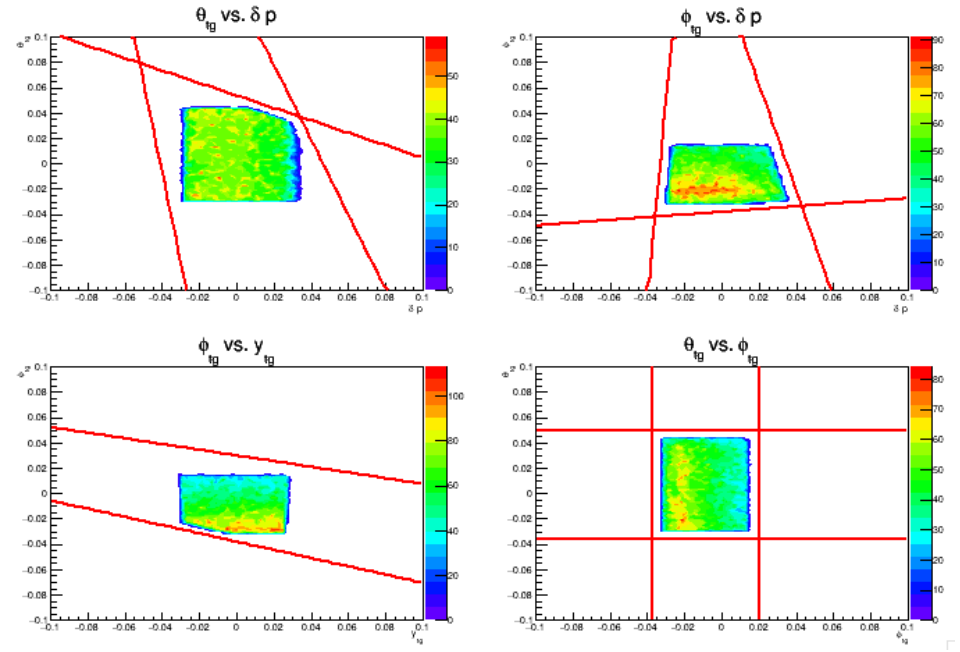

(b) after R-Function acceptance cut

Figure 3.8: Figures showing HRS data in target variables $\left\{y_{t g}(\mathrm{~m}), \theta_{t g}, \phi_{t g}, \delta p\right\}$ before and after an R-Cut with a positive value has been applied.

The R-Function (and the cuts used to define it) should not depend on the kinematic setting. However, because the Q1 quadrupole had a varying degree of functionality from setting to setting, a unique R-Function needed to be constructed for all of the Kin-48 settings, and one universal R-Function for the Fall 2016 settings (601, 603, 362, 363).

\subsection{Deciding on an ideal R-Cut}

It is important to note that the returned $\mathrm{R}$-value of an event is entirely dependent on the 2-D cuts used to define the R-function. For example, if the initial defining cuts were overly strict, many good events could be assigned negative R-Values. In this case, it would be advantageous to choose a negative R-Cut, that would still permit these events to pass the 
acceptance cut. Choosing an ideal R-Cut means maximizing statistics, while still maintaining a good acceptance region. The method used to find this ideal R-Cut, or R-Ideal, will be discussed in Section 4.1.1.5, 


\section{Chapter 4}

\section{The Experimental DIS Cross Section}

Deep inelastic scattering is a well studied reaction whose cross section has been measured for large ranges in $x_{B}$ and $Q^{2}$. The convenience of easily recording DIS events concurrently with DVCS running allows us to extract a DIS cross section from our data. A comparison to world data is used to confirm we understand our detectors and any of their associated inefficiencies.

\subsection{Extracting the DIS Cross Section}

The cross section of a certain scattering reaction is a quantity describing how likely that reaction is to occur. This quantity should be universal, in that it is independent of what lab, equipment, and techniques were used to measure it. A count rate extracted from our data then needs to be "normalized", a process by which all experimental dependencies are removed. For instance, the L-HRS in Hall A would measure a different DIS count rate than the CLAS detector in Hall B, because of the acceptance difference of the detectors. This is just one example of an experimental dependence that needs to be removed through normalization to extract a DIS cross section.

The normalized DIS cross section is given by:

$$
\frac{d^{2} \sigma}{d x d Q^{2}}=\frac{N_{D I S}}{\mathcal{L}} \cdot \frac{1}{\eta_{\text {exp }} \cdot \eta_{\text {virt }} \cdot \alpha \cdot \Gamma_{D I S}}
$$

Table 4.1 describes what each factor is, and whether it is found from the Monte Carlo simulation, experimental data, or theory predictions. Each parameter needs to be found for each of the different kinematic settings of the experiment. 


\begin{tabular}{|l|l|l|}
\hline Parameter & Method & Description \\
\hline$N_{D I S}$ & Data & Number of events surviving all of the analysis cuts \\
\hline $\mathcal{L}$ & Data & Integrated luminosity of the run \\
\hline$\eta_{\text {exp }}$ & Data & Accounts for inefficiencies and deadtimes of detectors \\
\hline$\eta_{v i r t}$ & Theory & Virtual radiative corrections factor \\
\hline$\alpha$ & MC & Real radiative corrections factor \\
\hline$\Gamma_{D I S}$ & MC & Phase space correction factor \\
\hline
\end{tabular}

Table 4.1: A table of important parameters used for extracting the DIS cross section.

\subsubsection{Analysis Cuts for $N_{D I S}$}

A single data run, typically one to two hours long, can yield 1,000,000 events. This data set includes both DVCS and DIS events, events from target cell windows, events where a pion triggered the Cherenkov detector, and many more scenarios. It's important when extracting the DIS cross section, to include only events that are DIS events. To guarantee this, many cuts are made to the initially large data set, and $N_{D I S}$ represents those that survive. The following sections detail the event selection cuts (trigger, particle tracking, particle identification, and acceptance cuts) used in our analysis.

\subsubsection{Trigger Cuts and Event Scaling}

Each data event recorded has a trigger pattern word associated with it, that notes what coincidence trigger caused the event. As discussed in Section 3.3, events with S2 \& Cer firing in coincidence are candidates for DIS. Additionally, S0 \& Cer events were also DIS candidates for all 48 kinematics, due to the timing of S0 and S2 signals discussed in Section 3.3.1. Once events are selected based on trigger, the prescale values are considered. For example, if the S2 \& Cer coincidence is prescaled by 2, then the HRS really saw twice as many events as were recorded with that trigger. These events then need to be scaled by 2 to account for the prescaling effects when finding $N_{a c c}$.

The treatment of the "Missing" DIS, S0 \& Cer, prescale was different. These events are caused by a signal in S0 reaching the trigger supervisor before the S2 signal, and the events are not tagged as S2 \& Cer, the standard DIS trigger. However, this can only happen when the S0 \& Cer coincidence is able to trigger an event. That is, if the S0 \& Cer prescale was set to 4 , only every fourth event with that coincidence would be recorded. Therefore, only every fourth event with that coincidence would even be able to "steal" from a normal S2 \& Cer standard DIS event, and these "Missing" DIS events are not scaled when added to the sum of $N_{a c c}$. 
$\delta p$

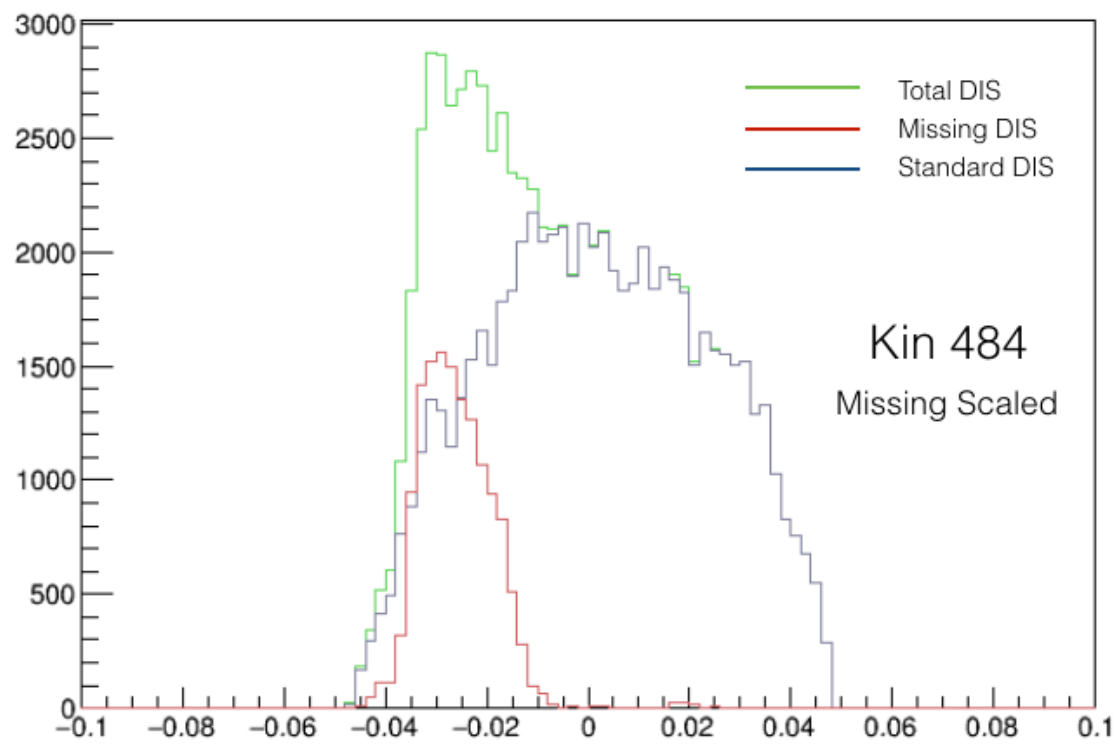

(a) $\delta p$ for Kin 484 , with missing events scaled

$\delta \mathrm{p}$

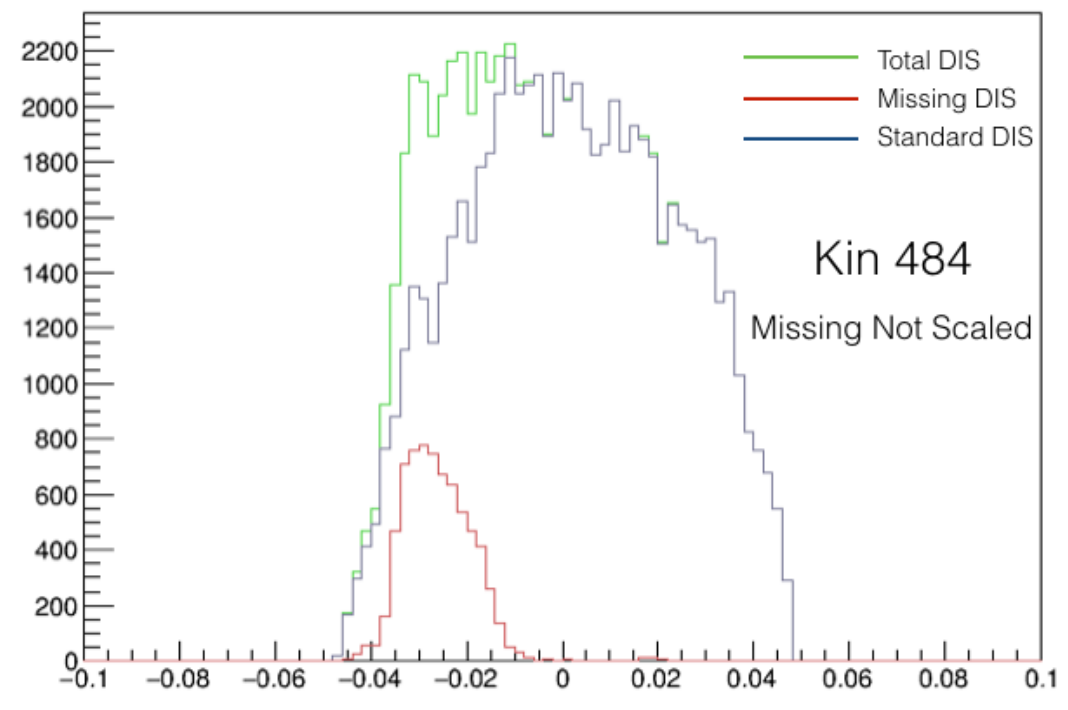

(b) $\delta p$ for Kin 484, without missing events scaled

Figure 4.1: Two figures showing the effects of scaling (a.) vs. not scaling (b.) "missing" DIS events by the S0 \& Cer prescale. For this kinematic setting, not scaling the events shows good agreement with simulation data (figure 4.2). 


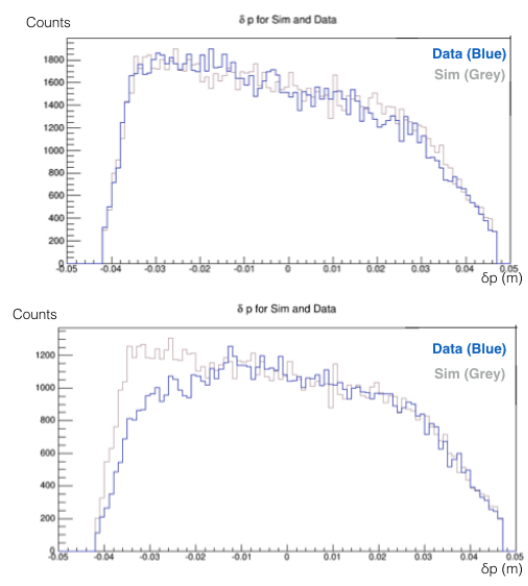

Figure 4.2: The $\delta \mathrm{p}$ distributions for the simulated data (grey) and experimental data (blue) for kinematic 484. The top figure shows the experimental data after including the missing events, without scaling by the S0 \& Cer prescale. The bottom figure shows the experimental without the missing events included.

To confirm that this is the correct treatment of prescales for the kinematic 48 settings, plots of $\delta p$ with different scaling of the S0 \& Cer prescale were compared for the same run. On the plots, "Missing not scaled" signifies that missing events were added to the total DIS with only a factor of one, while "Missing Scaled" means that missing events were added to the total DIS after being multiplied by the prescale value of the S0 \& Cer trigger for the specific run. It was found that for kinematics 482, 483, and 484, ignoring the prescale for "Missing" DIS events was the correct treatment. Figure 4.1 shows a sample run for kinematic 484, with and without scaling for the missing events. However, the opposite was true for kinematic 481, and scaling the missing events by the S0 \& Cer prescale was necessary for good agreement in the $\delta p$ plane. This comparison is shown in Figure 4.3. The cause of this discrepancy between the scaling outcome for kinematic 481 and 482, 483, and 484 is unknown. However, the discrepancy may be related to the actual prescale values used for the different settings. For example, kinematics 482, 483, and 484 had an S0 \& Cer prescale of 128 , while the S2M \& Cer (DIS) prescale was 2 or 4 . This is unlike kinematic 481, that had a prescale of 2 for both S0 \& Cer and S2M \& Cer (DIS).

However, the discrepancy may be related to the large prescale of 128 for the S0 \& Cer trigger and the DISfor the kin 482, 483, and 484 settings, and the smaller S0 \& Cer prescale of 2 for kinematic 481 . 


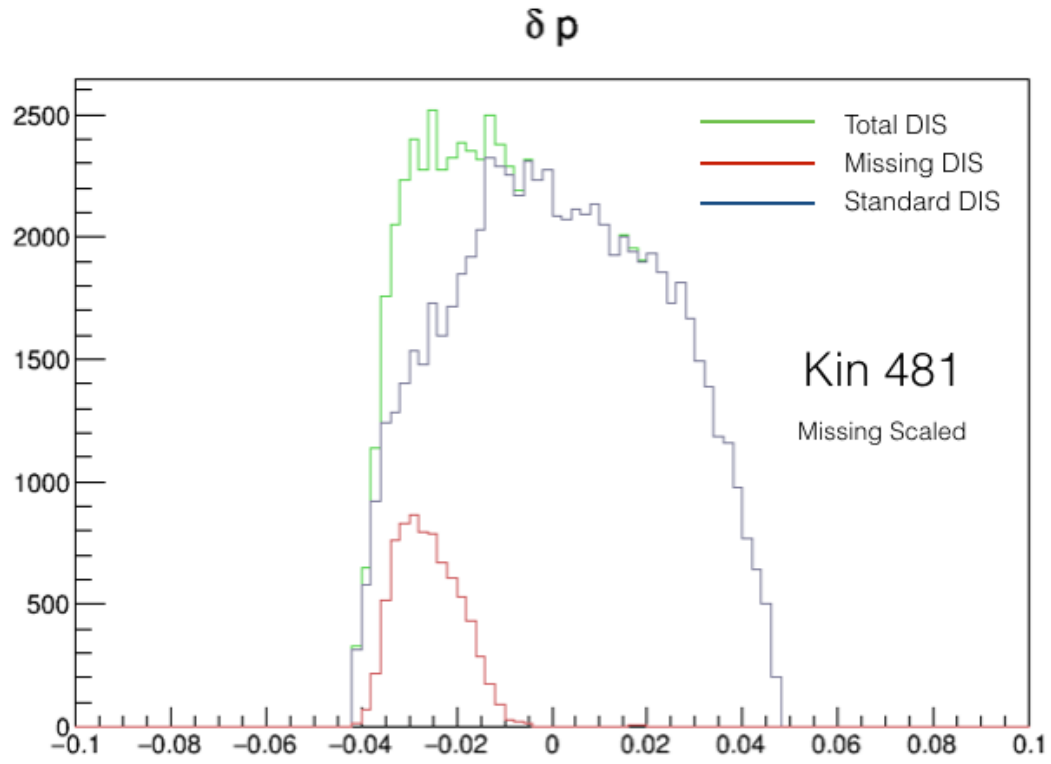

(a) $\delta p$ for Kin 481, with missing events scaled

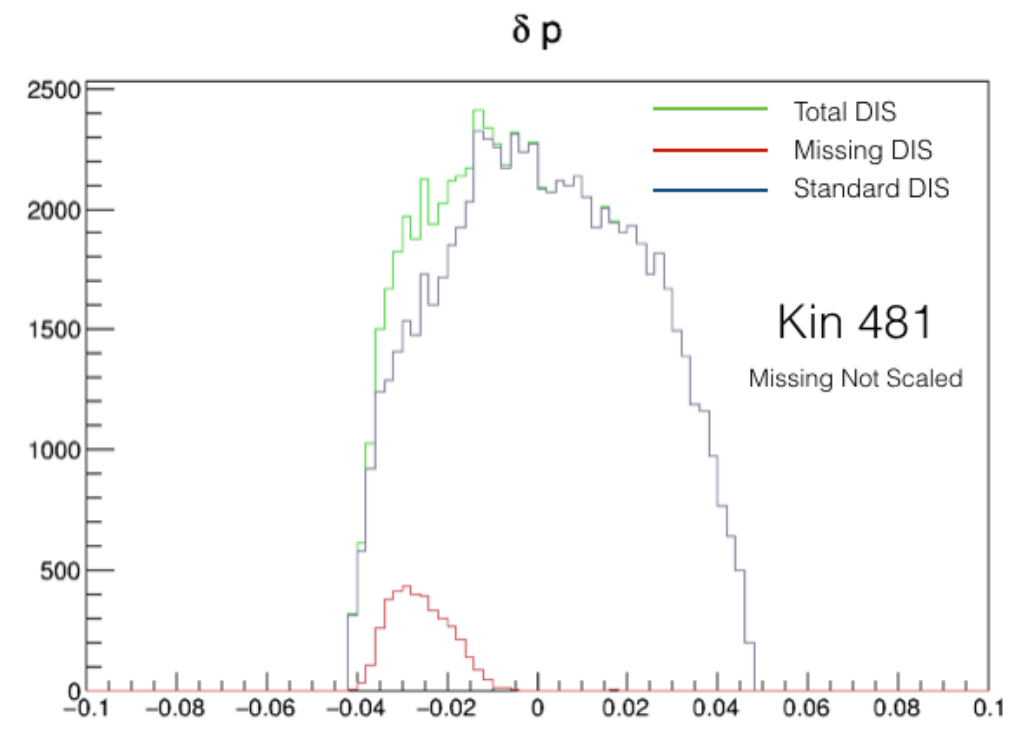

(b) $\delta p$ for Kin 481, without missing events scaled

Figure 4.3: Two figures showing the effects of scaling (a.) vs. not scaling (b.) "missing" DIS events by the S0 \& Cer prescale. For this kinematic setting, scaling the events does show good agreement with simulation data. 


\subsubsection{Target vertex}

The target used during DVCS production running, and therefore also the target used for DIS data, is a $15 \mathrm{~cm}$ long aluminum cylinder filled with $\mathrm{LH}_{2}$. We are not guaranteed that scattered particles originated from the $\mathrm{LH}_{2}$, as incoming electrons could have scattered off of the cell windows. A cut on the scattering vertex is made to eliminate these unwanted events. An identical, empty aluminum (dummy) target is used to study how much the cell windows contaminate the real data, and Figure 4.4 shows the reconstructed $\mathrm{z}$ vertex for a dummy run overlaid with production data. Table 4.2 lists the up and downstream vertex cuts used for each kinematic setting.

\section{'L.tr.vz' Dummy (12639) and Data (12518) for Kin 481}

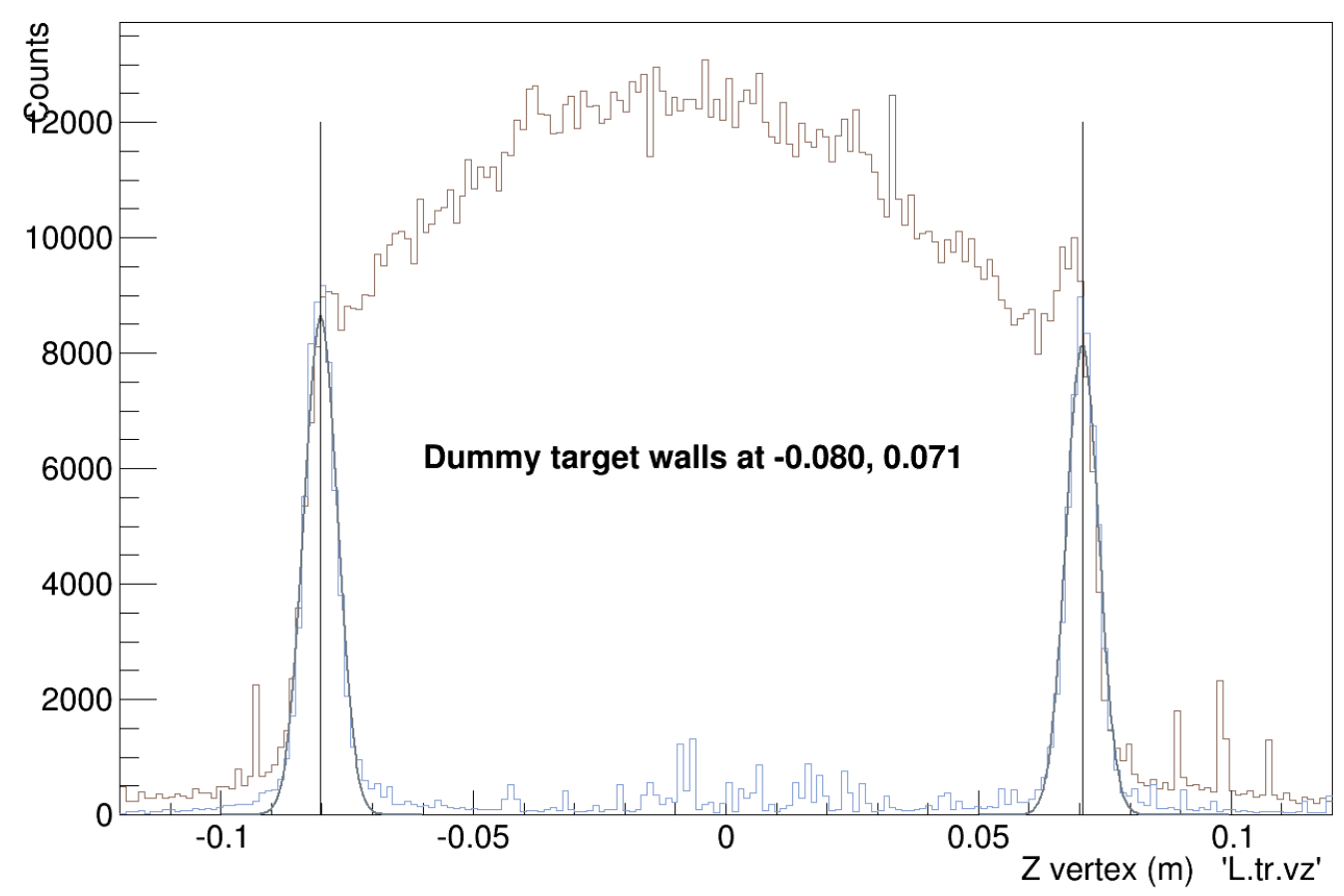

Figure 4.4: Production $\mathrm{LH}_{2}$ data overlaid with data from an empty dummy target, used to identify the contamination from target cell windows. Here the blue histogram shows the dummy target, and the brown shows the standard $\mathrm{LH}_{2}$ target. The black histograms estimate the location of the cell windows and their contamination on the experimental data. 


\begin{tabular}{|c|c|c|c|}
\hline \multicolumn{4}{|c|}{ Vertex Cuts } \\
\hline Setting & $z_{\text {up }}(\mathrm{cm})$ & $z_{\text {down }}(\mathrm{cm})$ & $\begin{array}{c}\text { Eff. Target } \\
\text { Length }(\mathrm{cm})\end{array}$ \\
\hline 361 & -5.75 & 7.25 & 13.00 \\
362 & -6.25 & 6.75 & 13.00 \\
363 & -5.50 & 7.50 & 13.00 \\
481 & -6.50 & 6.50 & 13.00 \\
482 & -6.25 & 6.75 & 13.00 \\
483 & -6.25 & 6.75 & 13.00 \\
484 & -6.00 & 7.00 & 13.00 \\
601 & -6.00 & 7.00 & 13.00 \\
603 & -6.25 & 6.75 & 13.00 \\
\hline
\end{tabular}

Table 4.2: A table of vertex cuts for each kinematic setting. The cuts differ for each kinematic setting because the target position changed.

\subsubsection{Particle identification}

The minimum momentum for particles to radiate energy in the gas Cherenkov detector is $17 \mathrm{MeV} / \mathrm{c}$ for electrons, and $4.8 \mathrm{GeV} / \mathrm{c}$ for pions. Using a signal in the gas Cherenkov as part of our trigger helps ensure we select on scattered electrons with just trigger cuts to the data. However, sometimes high momentum pions will produce signals in the Cherenkov due to knock-out electrons for example, and events will be recorded as if they were scattered electrons from the vertex. Therefore, we need to make additional cuts to remove this pion contamination, which involves cuts on both the Cherenkov signal, and both pion rejector $(\mathrm{PR})$ (section 2.2.3.3) layer ( $l 1$ and $l 2)$ signals. These are called particle identification, or PID, cuts. 


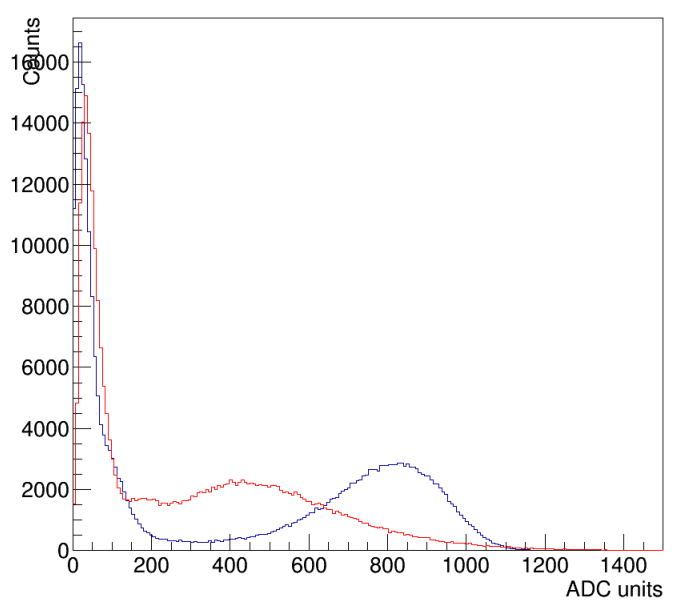

(a) PR layer signals before PID cuts

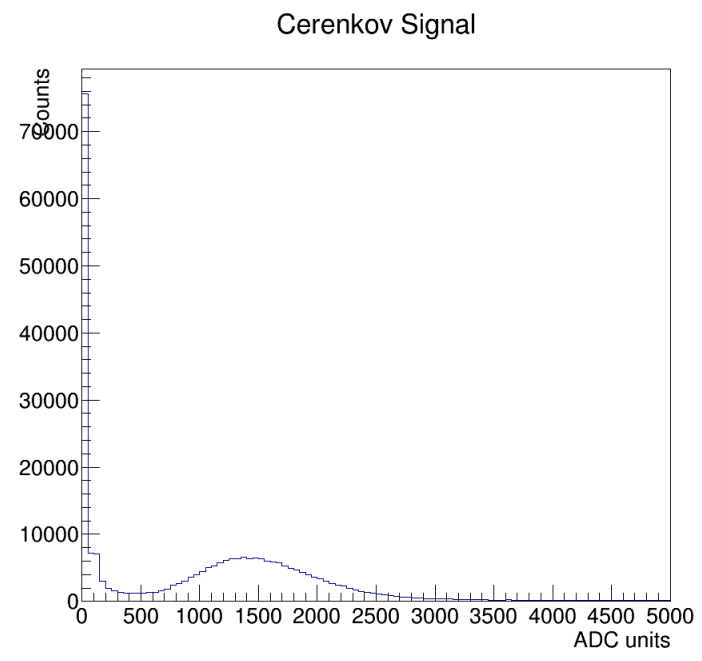

(b) Cherenkov signal before PID cuts

Figure 4.5: Figure (a.) shows the particle identification spectra for the pion rejector layer 1 (blue) and 2 (red), and figure (b.) shows the particle identification spectra for the Cherenkov detector. These spectra are from data before any cuts have been applied.

Figure 4.5 shows the Cherenkov and pion rejector signals before any PID cuts have been applied. Both layers of the PR array are shown, with smaller signals representing pion contamination. This is because electrons will deposit their full energy in the electromagnetic shower detector, and pions will not. Similarly, pions produce very low signals in the Cherenkov detector, and electrons produce the peak centered around 1300 ADC units.

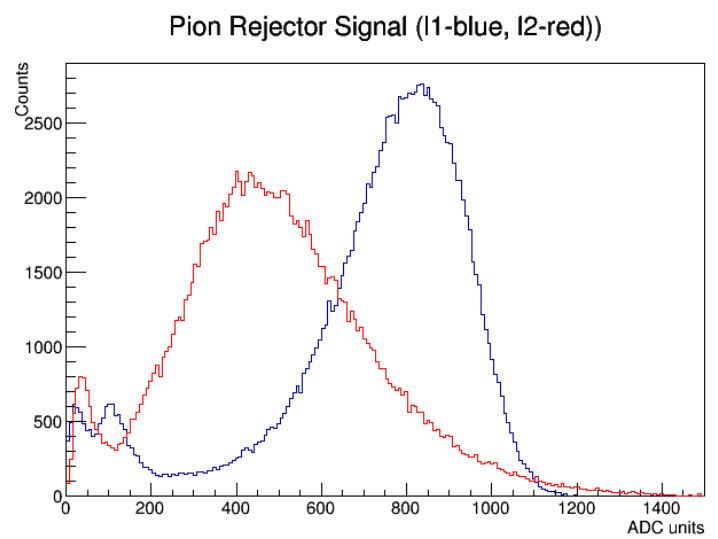

(a) PR layer signals with only Cherenkov cuts

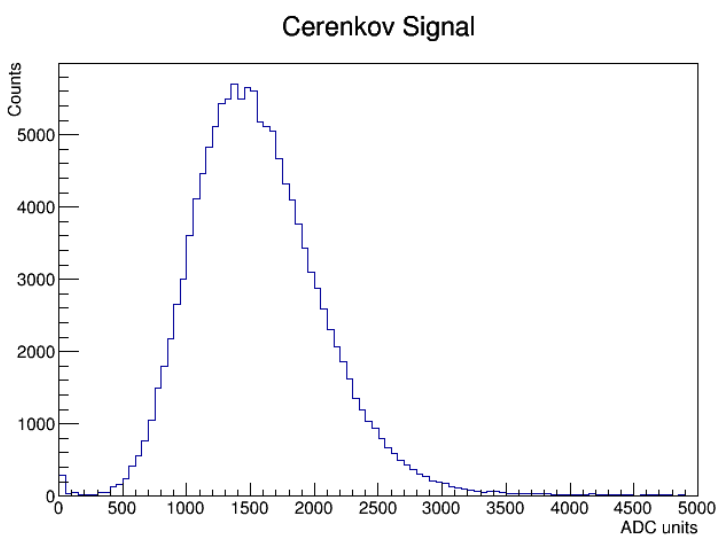

(b) Cherenkov signal with only $P R$ cuts

Figure 4.6: Figure (a.) shows the particle identification spectra for the pion rejector layers 1 and 2 (blue and red respectively) with only a Cherenkov signal cut, and figure (b.) shows the particle identification spectra for the Cherenkov detector with only a pion rejector cut. Using either cut alone is not sufficient to eliminate all pion contamination. 
Using only a cut determined from the Cherenkov spectrum, or only a cut from the PR spectrum, does not fully remove pion contamination. Figure 4.6 shows the PR layers with only a Cherenkov cut applied, and conversely, the Cherenkov spectrum with only a PR cut applied.

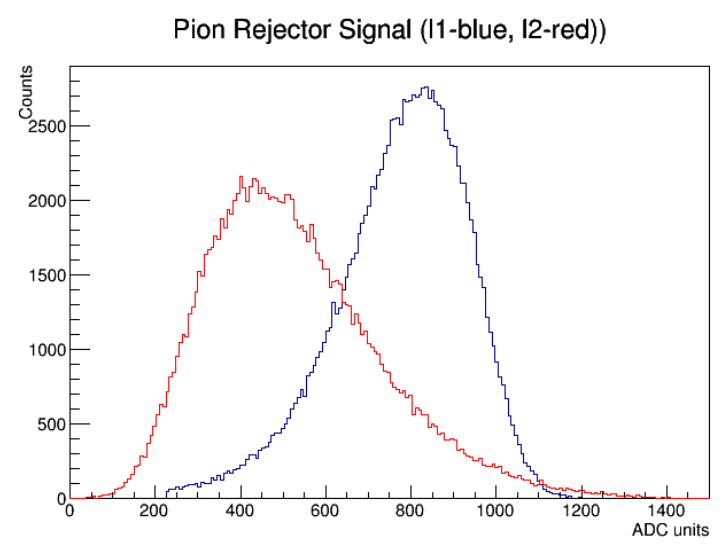

(a) PR layer signals with both PID cuts

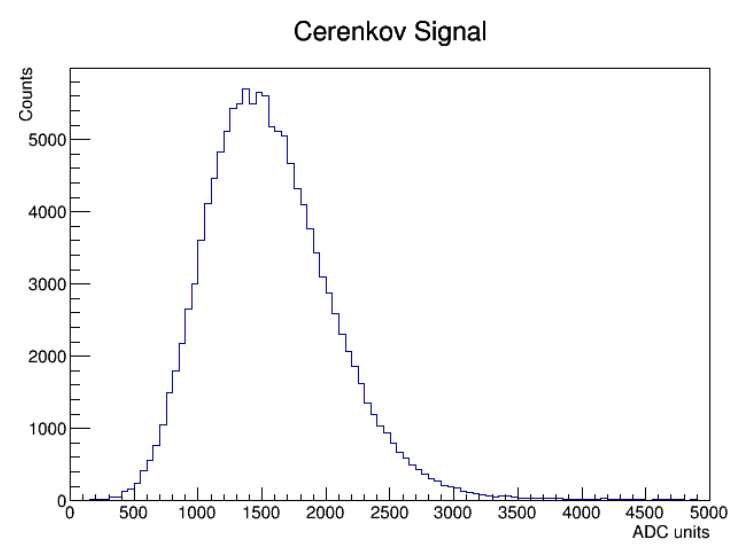

(b) Cherenkov signal with both PID cuts

Figure 4.7: Figure (a.) shows the particle identification spectra for the pion rejector layers 1 and 2 (blue and red respectively), and figure (b.) shows the particle identification spectra for the Cherenkov detector. Both pion rejector and Cherenkov cuts have been applied in both figures.

We instead use a combination of Cherenkov cuts (cer $\geq 100)$ and pion rejector cuts $\left(\operatorname{pr}_{l 1}\right.$ $+\operatorname{pr}_{l 2} \geq 600$ and $\operatorname{pr}_{l 1} \geq 200$ ). Figure 4.7 shows each spectrum after all PID cuts have been applied.

\subsubsection{Particle Tracking}

As electrons pass through the two VDCs, held in a constant electric field, the gas inside ionizes. The excess electrons from ionization will then travel to the sensing wires along a path of least time. As the electrons coalesce onto a sensing wire, a "cluster" is formed. In an ideal scenario, a single electron traveling through the two VDCs should produce a single cluster in each wire plane, allowing the particle trajectory to be reconstructed.

However, sometimes multiple clusters are seen in one or more wire planes, and multiple tracks can be reconstructed. If multiple clusters are seen in only one wire plane, only a single track will be reconstructed, and we do not reject the event. However, if more than one wire plane has multiple clusters, multiple particle trajectories (or multi-tracks) will be reconstructed. To avoid selecting the incorrect track, these multi-track events are discarded.

\subsubsection{Acceptance}

A cut is made on the data to accept only events included in an area of well-known HRS acceptance. The cut is performed via the R-Cut method (discussed previously in Section 3.4.3.1), 
which usually will be independent of kinematic setting. However, because the Q1 experienced varying degrees of dysfunction for Spring and Fall 2016 running, kinematic-specific R-Functions were used for all the Spring 2016 settings (481, 482, 483, 484), and one universal function for Fall 2016 (362, 363, 601, 603).

Once the R-Functions are determined for each setting, the R-Value of each event in a given run can be calculated. These R-Values will be positive or negative, depending on whether the events lie inside or outside of the acceptance region defined by the cuts. A value $\mathrm{R}$-Cut is chosen, so that events having an R-Value greater than or equal to the R-Cut will be accepted, and events with R-Value less than R-Cut will be discarded.

To determine the ideal R-Cut, R-Ideal, we extract a DIS cross section with some RCut. This is repeated for a large range in R-Cut (1000 data points between $-0.02,+0.02$ for example), and the value of the extracted cross section is observed. As the value of R-Cut increases, eventually we expect that all the accepted events are within a region of good acceptance, and the value of the extracted cross section should stabilize. This R-Cut is chosen to be R-Ideal, so that statistics are maximized while still maintaining a good acceptance region.

\section{Cross Section \% Diff vs. R-Cut}

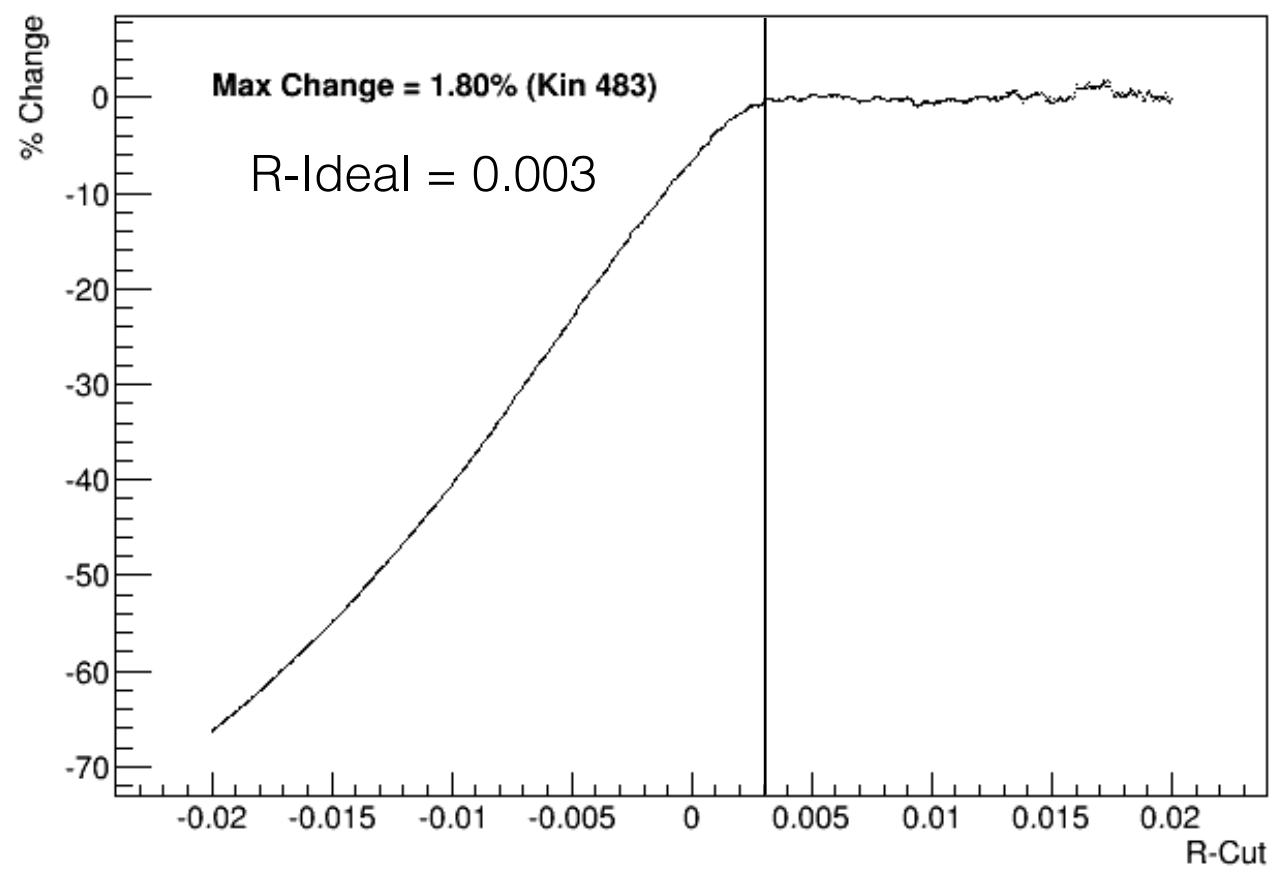

Figure 4.8: Extracted cross section (Kin 483) \% change (relative to the extracted value at R-Ideal $=0.003)$.

Figure 4.8 shows the extracted cross section (relative to the extracted value at R-Ideal $=$ 
0.003 (rad) for Kinematic 483) for a range in R-Cut values. At 0.003 radians, the extracted cross section stabilizes, with only a maximum fluctuation of $1.80 \%$ seen thereafter. This is repeated for all nine kinematic settings, with $\mathrm{R}$-Ideal being determined in this way. The maximum fluctuation of the extracted cross section seen above R-Ideal is then assigned as the systematic uncertainty associated with the R-Cut acceptance method. Table 4.3 displays the systematic uncertainty, R-Error, for each kinematic setting.

\begin{tabular}{|c|c|c|}
\hline \multicolumn{3}{|c|}{$\begin{array}{l}\text { R-Cut Systematic Uncertainty } \\
\text { R-Ideal }\end{array}$} \\
\hline Setting & R-Error & R-Ideal \\
\hline 361 & $1.02 \%$ & 0.003 \\
\hline 362 & $0.67 \%$ & 0.004 \\
\hline 363 & $1.15 \%$ & 0.004 \\
\hline 481 & $0.99 \%$ & 0.0045 \\
\hline 482 & $1.37 \%$ & 0.004 \\
\hline 483 & $0.96 \%$ & 0.003 \\
\hline 484 & $1.80 \%$ & -0.002 \\
\hline 601 & $2.23 \%$ & 0.004 \\
\hline 603 & $1.12 \%$ & 0.004 \\
\hline
\end{tabular}

Table 4.3: A table of R-Errors, the R-Cut uncertainty for each kinematic setting.

\subsubsection{Luminosity $\mathcal{L}$}

The integrated luminosity is effectively the number of incident particles, per cross-sectional area, the scattering target sees in a given run period. A higher luminosity corresponds to a larger number of incident particles on the target, and thus a higher chance for a scattering reaction to occur. Integrated luminosity can be found using:

$$
\mathcal{L}=N_{\text {incident }} \times\left(n_{\text {target }} \times l_{\text {target }}\right)
$$

Table 4.4 lists a description of the parameters used to find $\mathcal{L}$.

\begin{tabular}{|ll|}
\hline Parameter & Description \\
\hline$N_{\text {incident }}$ & Number of incident particles \\
\hline$n_{\text {target }}$ & Number density of target particles \\
\hline$l_{\text {target }}$ & Length of target \\
\hline
\end{tabular}

Table 4.4: Important parameters used for calculation of the integrated luminosity for a run. 
Experimentally, for a given run period, we only have access to the total charge $(Q$ in Coulombs), the temperature of the target $(19 \mathrm{~K})$, the target material $\left(\mathrm{LH}_{2}\right)$, the target length $(15 \mathrm{~cm})$, and the target pressure $(25 \mathrm{psi})$. We can use the pressure and temperature of the target to find the corresponding mass density of $\mathrm{LH}_{2}, \rho=0.073284 \frac{\mathrm{g}}{\mathrm{cm}^{3}}$.

To put these calculable quantities in the form above, we use:

$$
N_{\text {incident }}=\frac{Q}{e} \quad n_{\mathrm{LH}_{2}}=\frac{N_{A} \times \rho}{A_{H}}
$$

with $e=1.602 \cdot 10^{-19} \mathrm{C}$ the electron charge, $A_{H}=1.0079 \mathrm{~g} / \mathrm{mol}$ the atomic mass of hydrogen, $N_{A}=6.022 \cdot 10^{23} \mathrm{~mol}^{-1}$ Avogadro's number, and $\rho=0.07229 \mathrm{~g} / \mathrm{cm}^{3}$ the density of the $\mathrm{LH}_{2}$ target.

\subsubsection{Calculating Q}

There were 4 different beam current monitors (BCMs) active in Hall A during the experimental running, three downstream (with approximate prescales of 1, 3 and 10), and one upstream (prescale 1), of the target. The output voltage of each BCM goes through a voltage-to-frequency converter, and then into a scaler. The frequency of the BCM $\left(f_{B C M}\right)$ measured by the scalers is proportional to the current. The total counts seen by the end of a run will be proportional to the total charge sent to the target, $Q_{\text {total }}$. $Q_{\text {total }}$ was calculated for all four BCMs, using the same method for each.

In addition to a BCM scaler, Hall A has a clock scaler, whose constant frequency determines how many counts per second the clock scaler will record. It is important to note that the clock scaler will count regardless of beam conditions. So, the ratio of clock frequency to clock counts $\left(\frac{f_{\text {clock }}}{C_{\text {clock }}}\right)$ serves as a conversion factor to convert BCM counts $\left(C_{B C M}\right)$ to BCM frequency. This BCM frequency can then be compared to the Unser, to measure the precise gain $(G)$ and offset of the BCM. The current in each BCM is:

$$
I_{B C M}(t)=C_{B C M}(t) \times \frac{f_{\text {clock }}}{C_{\text {clock }}(t)} \times G+\text { Offset }
$$

From this equation we have:

$$
Q_{\text {total }}=Q\left(t_{\max }\right)=\int_{0}^{t_{\max }} I_{B C M}(t) \mathrm{d} t
$$

An example showing $Q(t)$ vs. $t$ for one BCM during a single run (Kin 481) is shown in Figure 4.9 . 


\section{d1 BCM Charge vs. Time(s)}

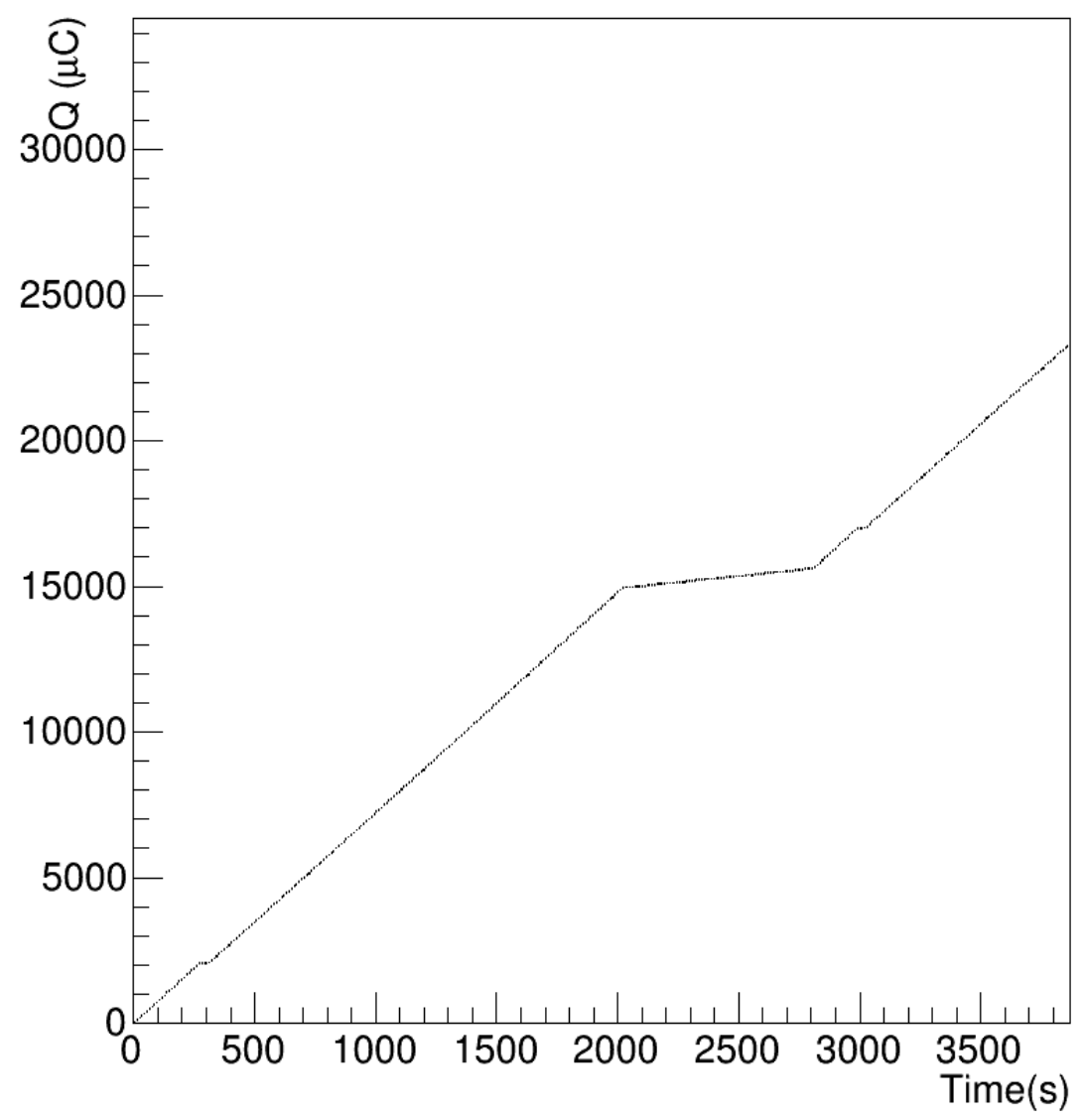

Figure 4.9: $Q(t)$ vs. $t$ found from BCM scaler d1 (downstream, prescale 1) counts during a single run.

Figure 4.10 shows the luminosity found for all runs of Kinematic 481. The value for each run depends on run length and beam current. 


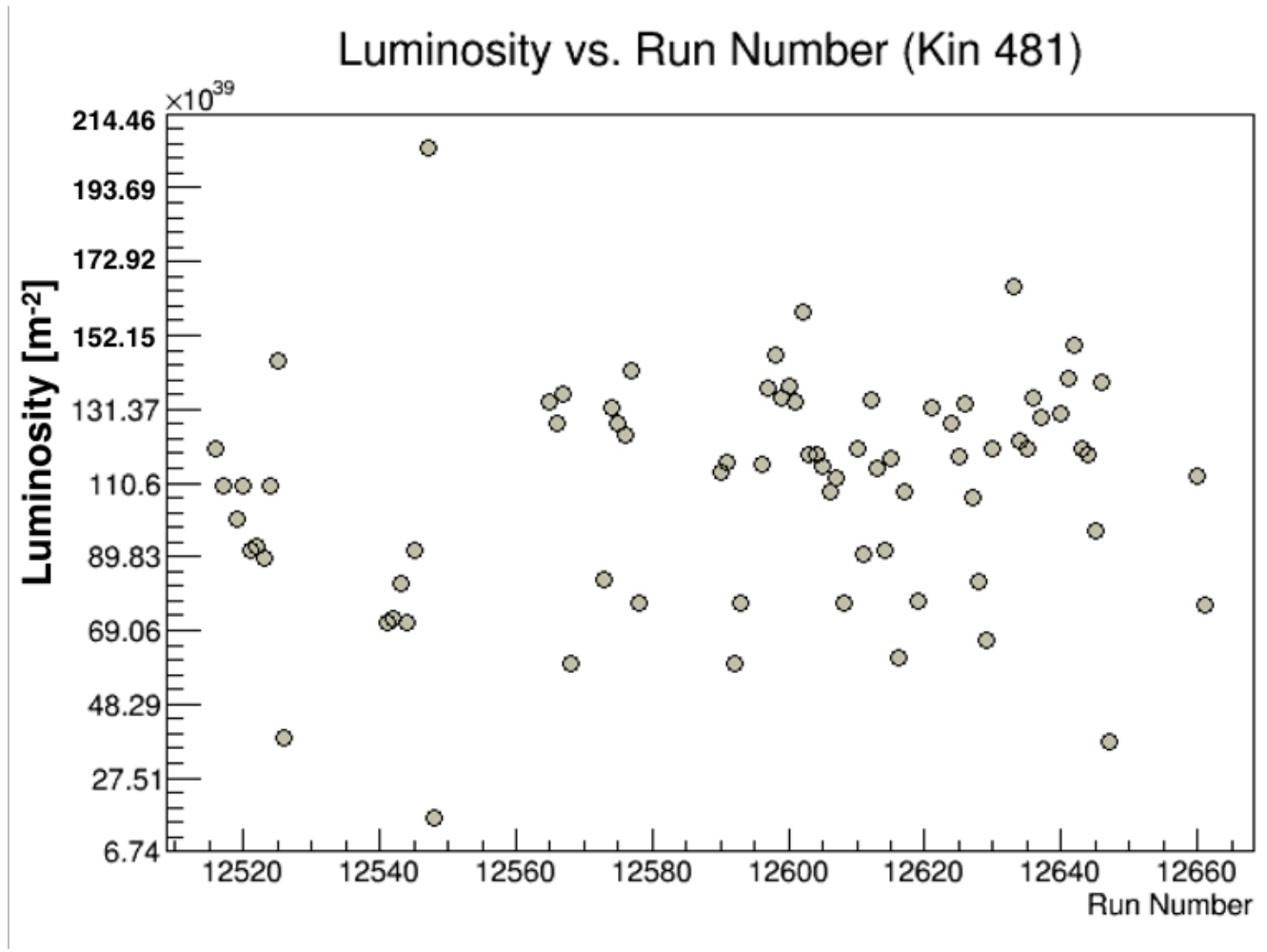

Figure 4.10: Luminosity values found for all runs of Kinematic 481. The scatter shown is related to how long the run lasted (typically anywhere between 30 minutes up to 2 hours).

\subsubsection{Efficiency and Deadtime correction $\eta_{\text {exp }}$}

The previous sections detailed all of the cuts used to reduce our data set to only DIS events. With all of these cuts, it is possible that good events were accidentally cut out (corresponding to efficencies $\eta_{\text {tracking }}$ and $\eta_{P I D}$ ), or missed completely by our detectors (corresponding to efficiencies $\eta_{C h e r}$ and $\eta_{S 2}$ ). The overall experimental efficiency correction factor is:

$$
\eta_{\text {exp }}=\eta_{D T} \cdot \eta_{P I D} \cdot \eta_{C h e r} \cdot \eta_{S 2} \cdot \eta_{T r a c k i n g}
$$

\subsubsection{Deadtime correction $\eta_{D T}$}

Scalers are used to find the deadtime of our detector system. When the DAQ is recording events, it cannot record any other events that may occur while the system is busy, resulting in a loss of accepted events. This is called deadtime, and it can be monitored with the use of two scalers: one that counts only when the DAQ is not busy (live scaler), and another that counts whether the DAQ is busy or not (raw scaler). So, the livetime of a given run can be found by comparing the resulting scaler counts:

$$
L T=\frac{\text { LiveScalerCounts }}{\text { RawScalerCounts }}
$$


The livetime is found and corrected for on a run by run basis. Figure 4.11 shows the livetime for each run of Kinematic 481. A deadtime of $1.5-2.3 \%$ is seen for most runs.

Livetime vs. Run Number (Kin 481)

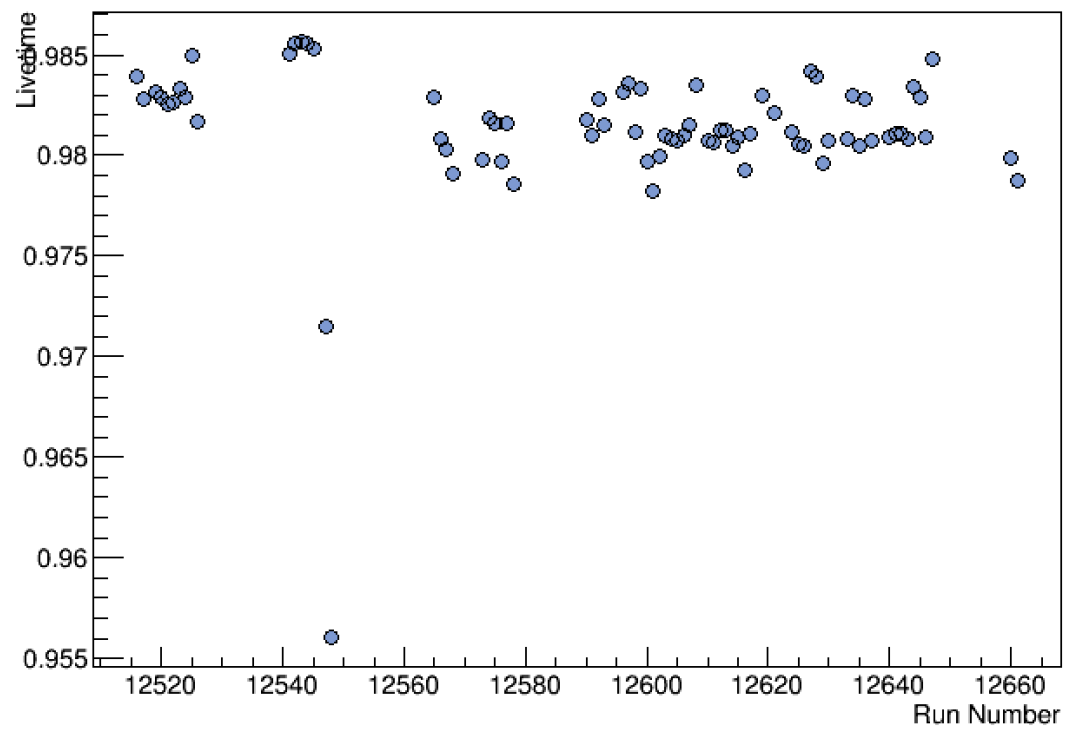

Figure 4.11: Livetime values found for all runs of Kinematic 481.

\subsubsection{2 $\eta_{\text {tracking }}$}

Section 4.1.1.4 discussed clusters and the functionality of the VDC chambers to provide tracking data of particles detected in the L-HRS. Ideally, only one track is reconstructed based on the timing of signals in sense-wires of the chambers (and ideally single-track events are correctly identified as being single-track). We begin by recognizing two types of "multicluster" events that can produce a single reconstructed track:

- $2 \mathrm{M} 2 \mathrm{~S}=2$ multi-clusters in the two planes of a VDC, and 2 single clusters in the two planes of the other VDC.

- $1 \mathrm{M} 3 \mathrm{~S}=1$ multi-cluster in a wire plane of either VDC, and 3 single clusters in the remaining planes.

There is a third case that is important to recognize but does not involve multi-clusters:

- $0 \mathrm{M} 4 \mathrm{~S}=$ a single cluster (and therefore zero multi-clusters) in each of the 4 wire planes.

The Hall A analyzer (v1.5) is known to have issues with reconstructing a track from a multi-cluster event. Some of the 2M2S, 1M3S, and 0M4S events are reconstructed to have multiple tracks, even though they never should. Additionally in the $2 \mathrm{M} 2 \mathrm{~S}$ case, due to 
the Hall A analyzer issues, a large portion of events having a single reconstructed track, show that track as being projected to miss the pion rejector completely (on the scale of whole meters), even though they are producing large signals in the pion rejector. All events showing multiple tracks are discarded, and all 2M2S events (regardless of whether or not it had a single reconstructed track) are discarded. We can define the number of events eliminated due to bad track reconstruction, that also pass particle identification cuts, in a given run as $N_{\text {Bad-tracking. }}$

If we assume all eliminated events should have had reliable single reconstructed tracks, and that events were only marked as bad due to Hall A analyzer software errors, then we must correct for the loss of good events. $N_{\text {Bad-tracking }}$ is compared to the total number of events passing the tracking cuts, and particle identification cuts, for a given run, $N_{\text {Good-tracking }}$. The tracking correction as it is applied as part of $\eta_{\exp }$ in the denominator of equation 4.1 is defined according to:

$$
\eta_{\text {Tracking }}=\frac{1}{1+\frac{N_{\text {Bad-tracking }}}{N_{\text {Good-tracking }}}}
$$

Like the livetime, the tracking efficiency correction is found and corrected for on a run by run basis. Figure 4.12 shows $\eta_{\text {Tracking }}$ for each run of Kinematic 481.

Tracking Efficiency vs. Run Number (Kin 481)

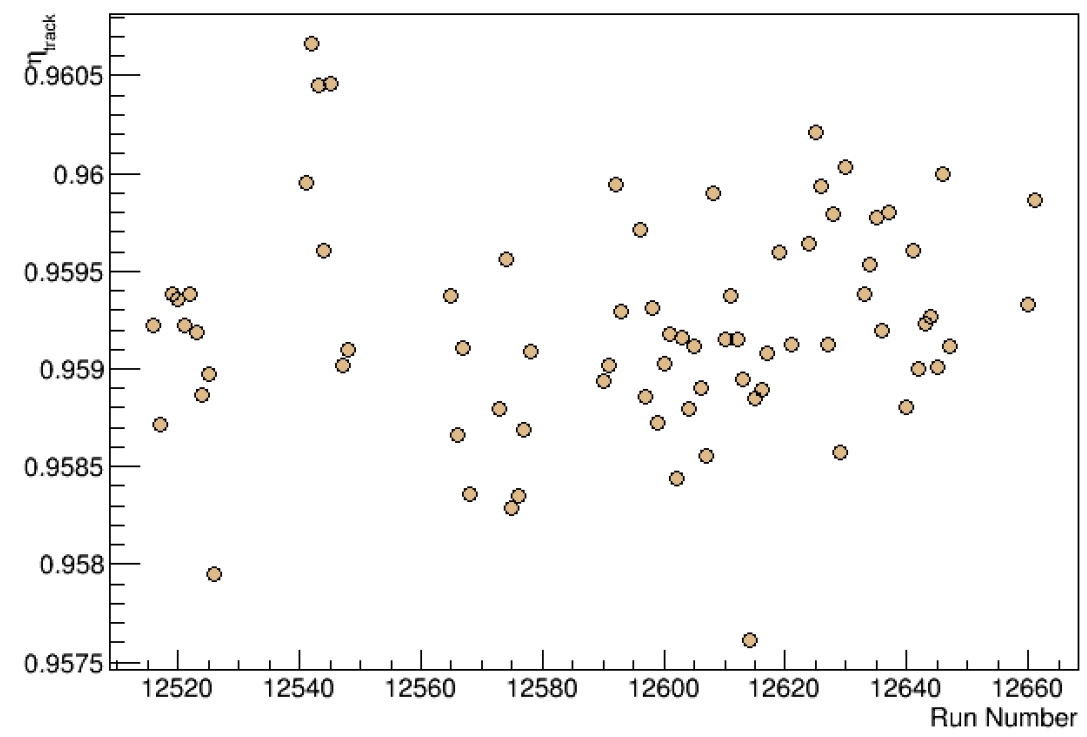

Figure 4.12: Tracking correction values found for all runs for Kinematic 481.

\subsubsection{3 $\eta_{\text {Cher }}$}

The efficiency of the gas Cherenkov trigger signal for Fall 2016 data is found using dedicated runs that do not require the Cherenkov detector in the trigger. It is found using the following expression: 
Where

$$
\text { GoodEvent }=\mathrm{S} 0 \mathrm{~S} 2 \mathrm{M} \cap \text { CherADC } \cap \mathrm{PR} \cap \mathrm{ST} \cap \text { Target } \cap \text { RFunc }
$$

With the following definitions:

- $\mathrm{S} 0 \mathrm{~S} 2 \mathrm{M}=\mathrm{A}$ cut that selects events where both S0 and S2M fired.

- CherTDC = A cut that selects events with good timing information from the TDC in the gas Cherenkov.

- $\mathrm{PR}=\mathrm{A}$ pion rejector cut that selects events identified as electrons using the pion rejector layers.

- CherADC = A cut that selects events identified as electrons using the gas Cherenkov's ADCs.

- $\mathrm{ST}=\mathrm{A}$ cut that selects single track events (at most one multi-cluster 1 in VDC planes $\mathrm{U} 1, \mathrm{U} 2, \mathrm{~V} 1, \mathrm{~V} 2$ ).

- Target $=\mathrm{A}$ cut that selects events within specified z-vertex target cut bounds, to guarantee events originated from the $\mathrm{LH}_{2}$ target.

- $\mathrm{RFunc}=\mathrm{A}$ cut that selects events within a well-understood region of L-HRS optics.

During Spring 2016 running, no dedicated efficiency runs were taken. Runs where the S0 \& S2M coincidence trigger was present were used (so the Cherenkov detector was excluded) to find the Cherenkov efficiency, but the runs had very low S0 \& S2M statistics. Still, the average value was $~ 99.7 \%$, which agrees with the Cherenkov efficiency results found with the dedicated runs in Fall 2016.

\subsubsection{4 $\eta_{S 2 M}$}

Because both the S0 and S2M scintillators can be used to signal a particle has passed through the HRS, a signal in either scintillator should indicate a signal seen in the other. To find the efficiency of the S2M scintillator, we need a run without S2M present in the trigger. Dedicated runs were taken in Fall 2016 that had no S2M trigger present. Even though S2M was not included in the trigger, it can and should still produce a signal for the DAQ to record, meaning all events triggered with S0 \& Cher for example, should have a signal present in S2M. For a given efficiency run, the number of events where S2M sees a particle, $N_{S 0 C h e r} \cap S 2 M$ can be compared to the set of all S0 \& Cher events, $N_{S 0 C h e r}$. The efficiency of the S2M scintillator is then:

\footnotetext{
${ }^{1}$ See section 4.1 .3 .2 for more details on multiclusters and tracking
} 


$$
\eta_{S 2 M}=\frac{N_{S 0 C h e r} \cap S 2 M}{N_{S 0 C h e r}}
$$

Unlike Fall 2016, Spring 2016 had no dedicated efficiency runs. Even though certain runs had the S0 \& Cher trigger present, all of these runs also had S2M in the trigger logic. However, calculations from both Fall and Spring (using the S0 \& Cer triggered events despite S2M being present in other triggers) saw similar $\eta_{S 2 M}$ values of $99.6 \%$.

\begin{tabular}{|c|c|c|c|c|c|}
\hline \multicolumn{6}{|c|}{ Approximate $\eta_{\exp }$ values for each Kinematic Setting } \\
\hline Setting & $\eta_{D T}(\%)$ & $\eta_{\text {tracking }}(\%)$ & $\eta_{\text {Cher }}(\%)$ & $\eta_{S 2 M}(\%)$ & $\eta_{\exp }(\%)$ \\
\hline 361 & 96.0 & 94.3 & 99.7 & 99.6 & 89.9 \\
362 & 96.5 & 94.0 & 99.7 & 99.6 & 90.1 \\
363 & 94.2 & 93.5 & 99.7 & 99.6 & 87.5 \\
481 & 98.3 & 95.9 & 99.7 & 99.6 & 93.6 \\
482 & 95.0 & 94.0 & 99.7 & 99.6 & 88.7 \\
483 & 97.2 & 94.6 & 99.7 & 99.6 & 91.3 \\
484 & 97.0 & 94.3 & 99.7 & 99.6 & 90.8 \\
601 & 97.6 & 93.8 & 99.7 & 99.6 & 90.9 \\
603 & 97.6 & 93.8 & 99.7 & 99.6 & 90.9 \\
\hline
\end{tabular}

Table 4.5: A table of experimental efficiencies for each kinematic setting.

\subsubsection{Monte Carlo Simulation}

A Monte Carlo simulation is used to find $\alpha$ and $\Gamma_{D I S}$ for each kinematic setting. An existing DVCS simulation was adapted for DIS running, and was used by my colleague Bishnu Karki in his DIS analysis. I, however, experienced slow run times using this method, so I developed a simplified DIS simulation entirely from scratch. Both simulations were used in the extraction of the DIS cross section and were found to be equivalent.

\subsubsection{Event Generator}

For a given kinematic setting, all events simulated begin with fixed beam energy $\left(E_{0}=E_{\text {beam }}\right.$ in Figure 4.13 below). A simulated event is first assigned a randomly generated scattering vertex $v_{z}, x_{B}$, and $Q^{2}$, chosen between $-7.5 \mathrm{~cm} \leq v_{z} \leq 7.5 \mathrm{~cm}, 0 \leq x_{B} \leq 1$, and $0 \leq$ $Q^{2} \leq 3 \cdot Q_{H R S}^{2}$, where $Q_{H R S}^{2}$ is the nominal HRS $Q^{2}$ from Table 3.1. Figure 4.13 shows the nomenclature of energy variables for each point in the simulation. 


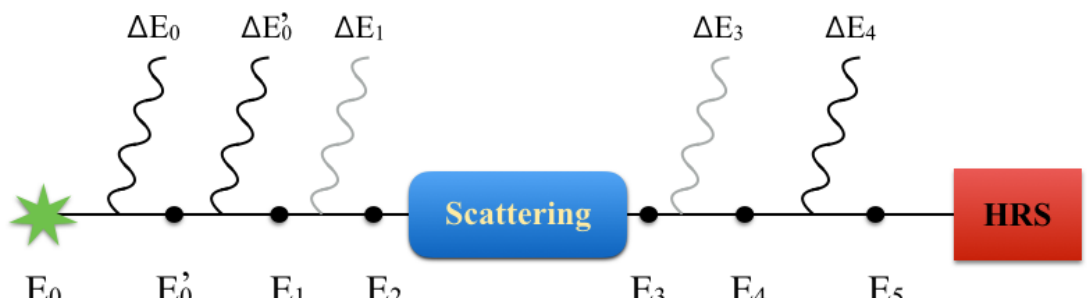

Figure 4.13: A graphic showing all energies and energy losses involved in the simulation. $\Delta E_{0}, \Delta E_{0}^{\prime}$, and $\Delta E_{4}$ are the real external radiative energy losses from traveling through the aluminum cell window and $\mathrm{LH}_{2}$ before scattering ( $\Delta E_{0}$ and $\Delta E_{0}^{\prime}$ respectively), and losses from traveling through the aluminum cell wall again after scattering $\left(\Delta E_{4}\right) . \Delta E_{1}$ and $\Delta E_{3}$ are the real internal radiative energy losses at the vertex before and after scattering.

From the initial $E_{0}$, external radiative losses from the aluminum cell window and $\mathrm{LH}_{2}$, $\Delta E_{0}$ and $\Delta E_{0}^{\prime}$ respectively, are found to yield $E_{1}$. Next, a real internal radiative loss at the vertex, $\Delta E_{1}$ is calculated, leaving $E_{2}$, the energy the electron has right before scattering. From knowing $x_{b}$ and $Q^{2}$ (and thus $\nu$ ), we can find $E_{3}$. Finally, the real internal radiative loss of the scattered electron, $\Delta E_{3}$ is found, along with the external radiative loss from exiting the aluminum cell wall, $\Delta E_{4}$, and $E_{5}$ is the energy that the HRS would see. Figure 4.14 shows these steps, and the following sections detail how the energy losses from radiative effects were calculated. Once $E_{5}$ has been found, all parameters can be inputted into the R-Functions developed for the kinematic setting, and R-Values are recorded. 


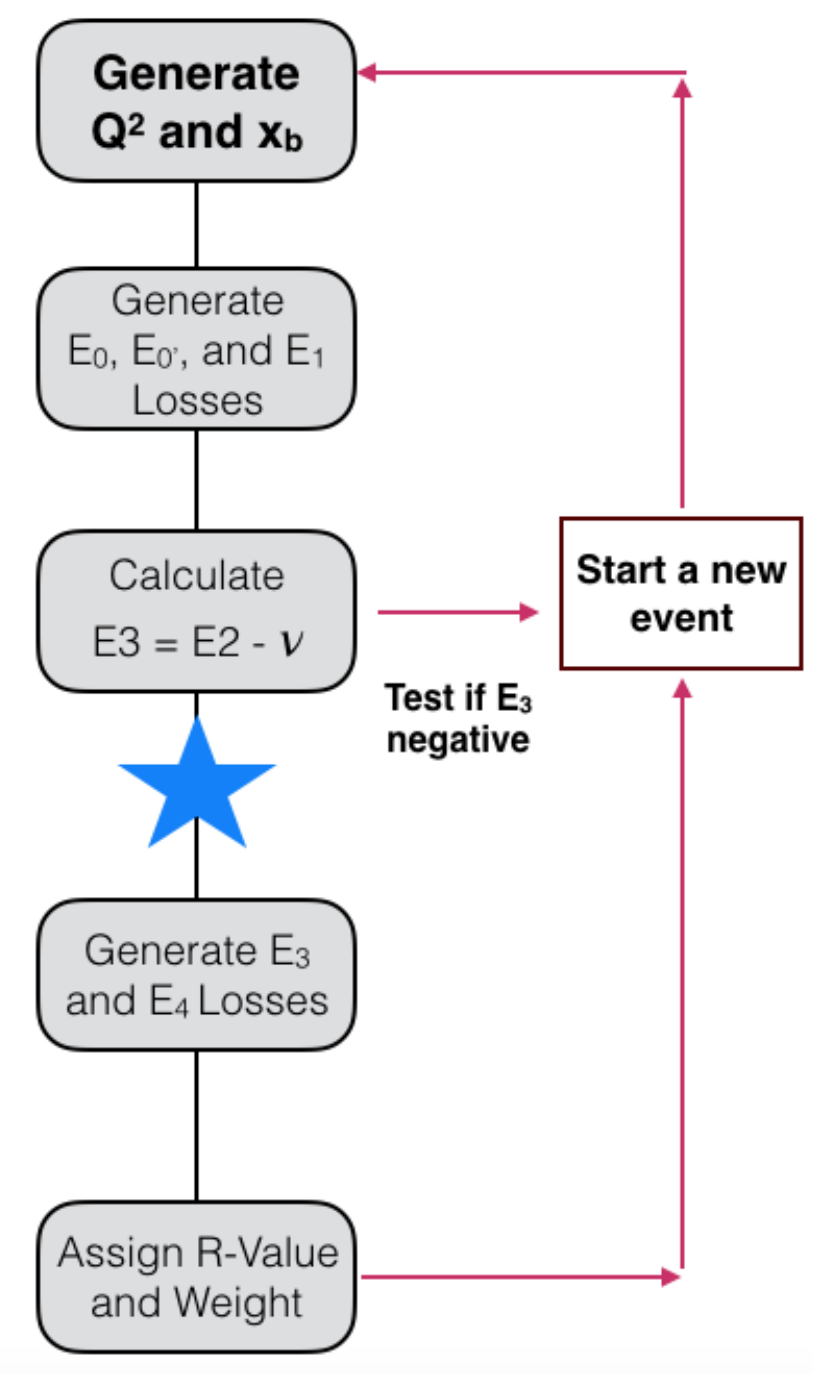

Figure 4.14: The steps of event generation for the simulation.

At this step, the simulation does not have the same distribution as DIS events, because $Q^{2}$ and $x_{B}$ were generated from flat distributions. Therefore, to make the simulation describe the DIS process, each event is assigned a "weight", which is the expected differential DIS cross section for the scattering kinematics $Q^{2}$ and $x_{B}$. These weights are found using a fit developed by Eric Christy and Peter Bosted [60] from a very large set of historical world data on the DIS cross section.

\subsubsection{External Radiative Energy Losses}

External radiative losses occur as electrons travel through material, in an effect known as Bremsstrahlung. For an incident energy $E, I_{e x t}$ is the distribution of the possible energy loss 
$\Delta E$ due to external radiative effects:

$$
I_{\text {ext }}\left(E, \Delta E, t_{m a t}\right)=\frac{b t_{m a t}}{\Delta E}\left[\frac{\Delta E}{E}\right]^{b t_{m a t}}
$$

with $b \approx \frac{3}{4}\left[69\right.$, and $t_{m a t}$ the number of radiation lengths of a given material. This distribution is generated using the relation

$$
\Delta E=E r^{1 / b t_{m a t}}
$$

Here, $b t_{\text {mat }}$ is the effective radiator thickness, and $r$ is generated randomly between 0 and 1. This relationship can be used to find the energy loss for every event of the simulation, as the electron passes through first the aluminum target cell window, and next the $\mathrm{LH}_{2}$ of the target.

Beginning with $E_{0}$, energy losses occur as the incoming electron passes through the aluminum target cell window. The energy loss, $\Delta E_{0}$ can be found according to

$$
\Delta E_{0}=E_{0} r^{1 / b t_{m a t}}
$$

where $t_{\text {mat }}$ is the length of the aluminum cell window in radiation lengths. The electron energy after this loss is then $E_{0}^{\prime}=E_{0}-\Delta E_{0}$. Note that the same expression is used for finding the energy loss $\Delta E_{4}$ as the outgoing electron again passes through the aluminum cell wall, with $E_{4}$ replacing $E_{0}$.

After the electron has passed through the target cell window, it moves through a portion of the $\mathrm{LH}_{2}$ before scattering off a proton. The radiative loss is again simulated according to

$$
\Delta E_{0}^{\prime}=E_{0}^{\prime} r^{1 / b t_{m a t}}
$$

with $t_{\text {mat }}$ now the number of radiation lengths of the $\mathrm{LH}_{2}$ traversed by the electron (chosen randomly from the total $15 \mathrm{~cm}$ total target length) before scattering. Thus $E_{1}=E_{0}^{\prime}-\Delta E_{0}^{\prime}$.

\subsubsection{Internal Radiative Energy Losses}

After external radiative corrections have been applied to the simulation, radiative effects at the scattering vertex are considered (referred to as internal radiative effects) [68] [69] [70] [71. Figure 4.15 shows three possible scenarios:

- a). Vertex correction: Before scattering, the incident electron emits a photon that then gets reabsorbed by the scattered electron.

- b). Vacuum polarization: The virtual photon turns into an electron-positron pair.

- c). Internal Bremsstrahlung: The incident or outgoing electron emits a real photon. 

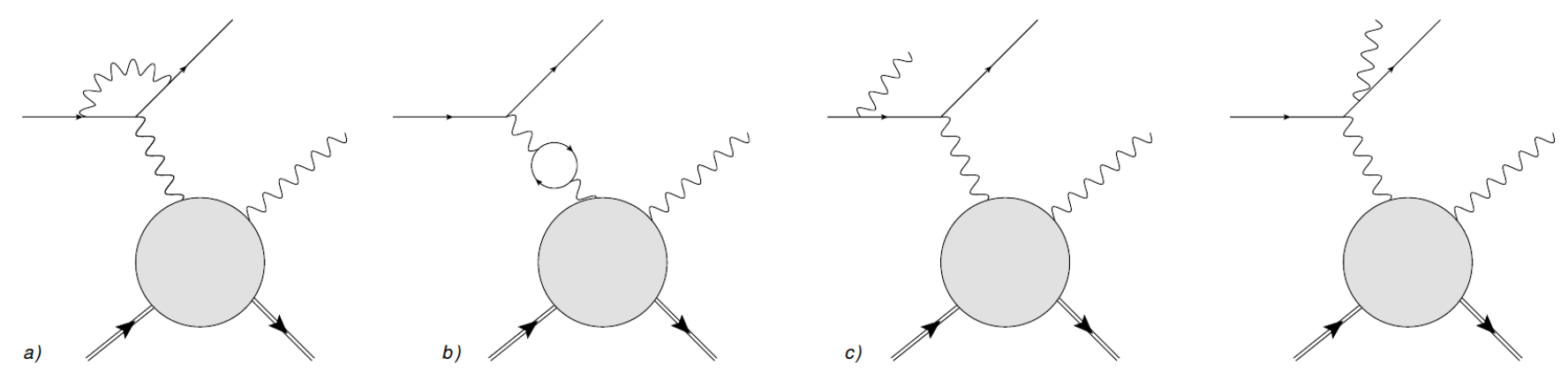

Figure 4.15: Diagrams showing possible internal radiative energy losses.

Using the calculations of Vanderhaeghen et al. [69], the modification of the Born cross section $d \sigma_{\text {Born }}$ from the three internal radiative corrections in figure 4.15) can be written in terms of quantities $\delta_{v}, \delta_{v a c}$, and $\delta_{R}$, for the vertex correction, vacuum polarization, and internal bremsstrahlung respectively. The resulting experimental cross section $d \sigma_{\exp }$ that includes radiative events with radiated photons of energy $\Delta E$ and $\Delta E^{\prime}$ can be written as:

$$
d \sigma_{e x p}\left(\Delta E, \Delta E^{\prime}\right)=d \sigma_{\text {born }} \frac{e^{\delta_{R}+\delta_{v e r}}}{\left(1-\delta_{\text {vac }}\right)^{2}}=d \sigma_{\text {Born }} \frac{e^{\delta_{v e r}}}{\left(1-\delta_{\text {vac }}\right)^{2}} e^{\delta_{R}^{(0)}}\left(\frac{\Delta E}{E}\right)^{\delta_{R}^{(1)}}\left(\frac{\Delta E^{\prime}}{E^{\prime}}\right)^{\delta_{R}^{(1)}}
$$

where:

$$
\begin{aligned}
& \delta_{R}^{(0)}=\frac{\alpha}{\pi}\left[S p\left(\cos ^{2} \frac{\theta}{2}\right)-\frac{\pi^{2}}{3}+\frac{1}{2} \ln ^{2}\left(\frac{Q^{2}}{m_{e}^{2}}\right)\right] \\
& \delta_{R}^{(1)}=\frac{\alpha}{\pi}\left[\ln \left(\frac{Q^{2}}{m_{e}^{2}}\right)-1\right] \\
& \delta_{\text {vac }}=\frac{2 \alpha}{3 \pi}\left[\ln \left(\frac{Q^{2}}{m_{e}^{2}}\right)-\frac{5}{3}\right] \\
& \delta_{\text {ver }}=\frac{\alpha}{\pi}\left[\frac{3}{2} \ln \left(\frac{Q^{2}}{m_{e}^{2}}\right)-2+\frac{\pi^{2}}{6}-\frac{1}{2} \ln ^{2}\left(\frac{Q^{2}}{m_{e}^{2}}\right)\right]
\end{aligned}
$$

with $\alpha$ the fine structure constant and $S p$ the Spence function.

The quantity $\left(\frac{\Delta E}{E}\right)^{\delta_{R}^{(1)}}$ terms represent the internal Bremsstrahlung case, before $(E)$ and after $\left(E^{\prime}\right)$ scattering. These can be interpreted as the fraction of incoming (outgoing) electrons that have lost an energy between 0 and $\Delta E\left(\Delta E^{\prime}\right)[69$.

Similar to the case of external Bremsstrahlung, the distribution of energy loss $(\Delta E)$ with initial energy $E$, and radiator thickness $\delta_{R}^{(1)}$ :

The internal radiative energy losses of the incoming and outgoing electrons, $\Delta E_{1}$ and $\Delta E_{3}$ in figure 4.14, are described by distributions $I_{\text {int }}(E, \Delta E)$ where 


$$
\int_{0}^{\Delta E} I_{i n t}(E, \Delta E)=\left(\frac{\Delta E}{E}\right)^{\delta_{R}^{(1)}}
$$

with

$$
\int_{0}^{E} I_{\text {int }}(E, \Delta E)=1
$$

therefore

$$
I_{\text {int }}(E, \Delta E)=\frac{\delta_{R}^{(1)}}{\Delta E}\left(\frac{\Delta E}{E}\right)^{\delta_{R}^{(1)}}
$$

Equation 4.15 can then be re-written in a more suitable form for the Monte Carlo:

$$
d \sigma_{\text {exp }}\left(\Delta E, \Delta E^{\prime}\right)=\int \Delta E_{1} \int \Delta E_{3} \frac{e^{\delta_{v e r}}}{\left(1-\delta_{v a c}\right)^{2}} e^{\delta_{R}^{(0)}} d \sigma_{\text {Born }} I_{\text {int }}\left(E_{1}, \Delta E_{1}\right) I_{\text {int }}\left(E_{3}, \Delta E_{3}\right)
$$

\subsubsection{4 $\eta_{\text {virt }}$}

The remainder of terms in equation 4.15, after the real internal Bremsstrahlung contributions are removed, leaves us with the correction to the Born cross section:

$$
\eta_{v i r t}=\frac{e^{\delta_{R}^{(0)}+\delta_{v e r}}}{\left(1-\frac{\delta_{v a c}}{2}\right)^{2}}
$$

Because the correction factor $\eta_{v i r t}$ is applied as an overall correction to the Born cross section, and each of the terms depends on $Q^{2}$, the nominal HRS setting $Q^{2}$ is used in the calculation.

Table 4.6 below shows the results of finding $\eta_{v i r t}$ (at nominal HRS kinematics) for each kinematic setting:

\begin{tabular}{|c|c|}
\hline \multicolumn{2}{|c|}{$\eta_{\text {virt }}$ Values } \\
\hline Setting & $\eta_{\text {virt }}$ \\
\hline 361 & 1.077 \\
362 & 1.078 \\
363 & 1.079 \\
481 & 1.076 \\
482 & 1.079 \\
483 & 1.080 \\
484 & 1.082 \\
601 & 1.080 \\
603 & 1.083 \\
\hline
\end{tabular}

Table 4.6: A table of $\eta_{\text {virt }}$ for each kinematic setting. 


\subsubsection{Non-physical Events}

Because $Q^{2}$ and $x_{b}$ are generated before any energies are found, we already have $\nu=\frac{Q^{2}}{2 M_{p} x_{b}}$. This is the same $\nu$ that represents the lab-frame energy loss in scattering, so in my simulation, $E_{3}$ is found using $E_{3}=E_{2}-\nu$. Because $E_{2}$ was found completely independently of $Q^{2}$ and $x_{b}$, it is possible that $E_{2}<\nu$ giving a negative value for $E_{3}$. Events that result in a negative $E_{3}$ are discarded for being non-physical.

\subsubsection{Radiative Effects on $N_{D I S}$}

Experimentally, we only have access to the electron beam energy ( $E_{0}$ in the simulation), and the scattered electron energy seen by the HRS ( $E_{4}$ in the simulation). We cannot see the radiative energy losses, and therefore do not have access to energies $E_{2}$ and $E_{3}$. This leads to an effect where the measured values of $x_{b}$ and $Q^{2}$ that the HRS sees for each scattering event are not necessarily the actual $x_{b}$ and $Q^{2}$.

For example, for kinematic 481, electrons have an initial beam energy of $4.483 \mathrm{GeV}$, and the central momentum setting of the HRS is $1.485 \mathrm{GeV}$. The range of $x_{b}$ and $Q^{2}$ from DIS, seen by the HRS (at angle $\theta_{H R S}=37.14^{\circ}$ ) is $0.42 \leq x_{b} \leq 0.54$ and $2.4 \leq Q^{2} \leq 3$. However, if an event were to lose a large amount of energy before scattering (from radiative effects), the HRS would measure a much larger $Q^{2}$ than was actually associated with the scattering. Similarly, if an event were to lose a large amount of energy after scattering, the HRS would measure a much smaller $Q^{2}$ than was actually associated with the scattering. It is also possible that radiative losses could push events out of acceptance. For instance, there are cases for which scattering events occur with $Q^{2} \approx Q_{H R S}^{2}$, but radiative effects after scattering would result in an energy too small to be seen by the HRS. 

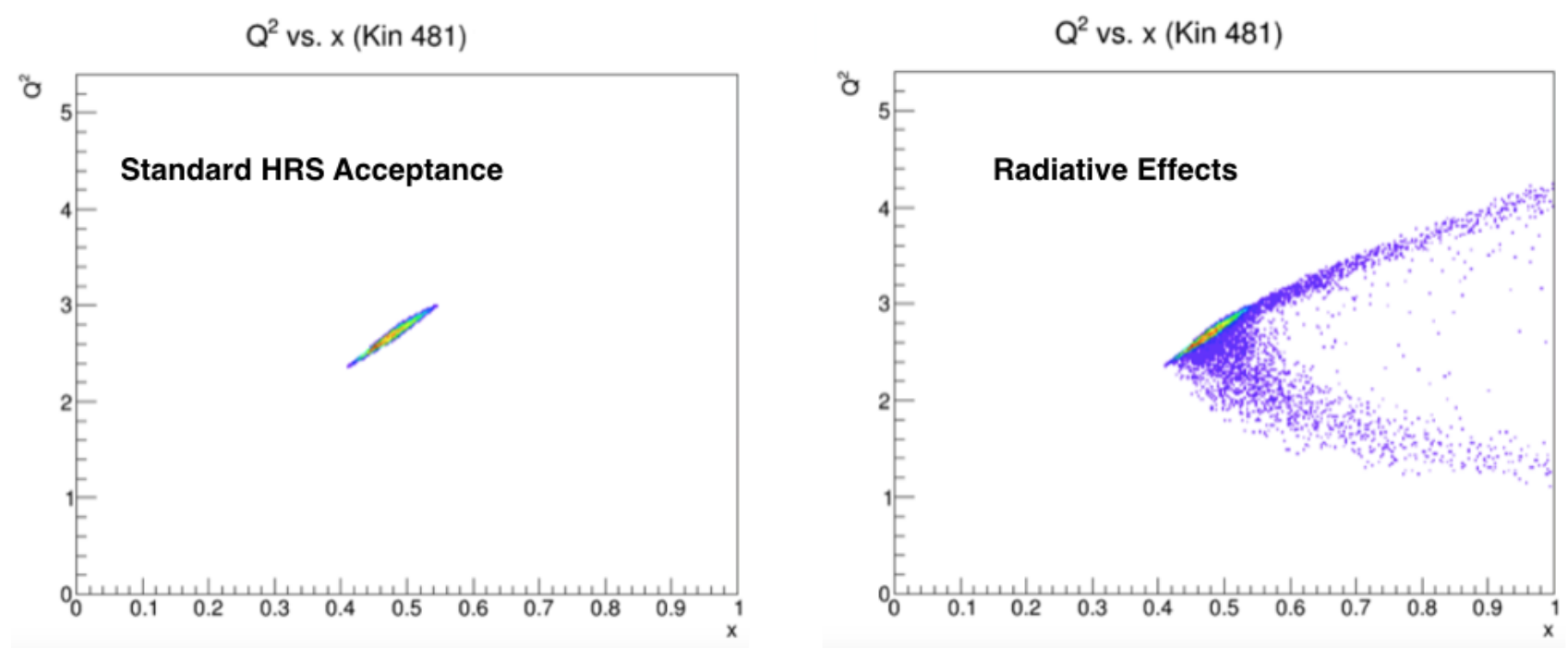

Figure 4.16: Effects of radiative energy losses on the acceptance of the HRS. The figure on the left shows the standard $Q^{2}$ vs. $x_{b}$ phase space of the HRS acceptance, the only values the HRS can measure. The figure on the right shows the larger $Q^{2}$ vs. $x_{b}$ range that can actually be accepted by the HRS due to radiative effects.

This effect is shown in Figure 4.16 above. The figure on the left shows the standard $Q^{2}$ vs. $x_{b}$ phase space of the HRS acceptance, the only values the HRS can measure. The figure on the right shows how a larger range of $x_{b}$ and $Q^{2}$ can actually be accepted, as these events in the bands would be measured to have $x_{b}$ and $Q^{2}$ values inside of the standard acceptance. When we extract an experimental cross section from our data, we need to apply corrections that account for these radiative effects. There are two separate corrections we need to apply: $\alpha$ and $\Gamma_{D I S}$.

\subsection{Radiative correction $\alpha$}

Ideally, if we are to extract a differential cross section $\frac{d^{2} \sigma}{d x d Q^{2}}(H R S)$, all events would have scattered with $x_{H R S}$ and $Q_{H R S}^{2}$. However, figure 4.16 (left) showed that a range of $x_{b}$ and $Q^{2}$ values can be accepted by the HRS. In fact this effect becomes even larger in the presence of radiative effects (figure 4.16 (right)), that can push events into acceptance with $x_{b}$ and $Q^{2}$ even farther away from the ideal $x_{H R S}$ and $Q_{H R S}^{2}$. The correction $\alpha$ is applied to the measured cross section (equation 4.1) to account for this effect.

\subsection{Phase space correction $\Gamma_{D I S}$}

The second correction, $\Gamma_{D I S}\left(\left(\right.\right.$ equation 4.1) ) accounts for the range in $x_{b}$ and $Q^{2}$ that recorded events are originating from, and is often referred to as the HRS phase space correction. It accounts for events that should have been included in our data set $N_{D I S}$ if the HRS acceptance were large enough to be able to detect them. The correction is necessary even 
without radiative effects, but the actual value will change as radiative effects begin pushing events into and out of acceptance. The following section details how both $\alpha$ and $\Gamma_{D I S}$ are found using simulated data.

\subsubsection{Calculating $\alpha$ and $\Gamma_{D I S}$}

The previous section detailed the motivations for needing correction factors $\alpha$ and $\Gamma_{D I S}$. In the following derivation, $\frac{d^{2} \sigma}{d x d Q^{2}}{ }_{\text {Kin })}$ is the cross section for an event with general scattering kinematics $x$ and $Q^{2}, \mathcal{L}$ is an integrated luminosity, and $\mathcal{C}$ is a term correcting for all experimental inefficiencies.

For each simulated event $i$ (with total simulated events being $N_{\text {gen }}$ ), all energies and energy losses are known $\left(E_{0}, \Delta E_{0}, E_{0}^{\prime}, \Delta E_{0}^{\prime}, E_{1}, \Delta E_{1}, E_{2}, E_{3}, \Delta E_{3}, E_{4}, \Delta E_{4}\right.$, and $E_{5}$ from figure 4.13), scattered parameters $x_{b, i}$ and $Q_{i}^{2}$, and the scattering cross section for each

individual event, $\left(\frac{d^{2} \sigma}{d x d Q^{2}}\right)$, are also known. All events in the simulation have a calculated R-Value $\left(r_{v a l}\right)$, to be used with a corresponding step function:

$$
\Theta\left(r_{\text {val }}-r_{c u t}\right)= \begin{cases}0 & r_{v a l} \leq r_{c u t} \\ 1 & r_{v a l} \geq r_{c u t}\end{cases}
$$

Experimentally, the total number of DIS events we expect to see in the HRS, when radiative effects are present, is given by the following expression:

$$
\begin{array}{r}
N_{D I S}=\eta_{\text {virt }} \mathcal{L} C \int_{-\frac{L}{2}}^{\frac{L}{2}} \frac{d z}{L} \int d\left(\Delta E_{0}\right) I_{\text {ext }}\left(E_{0}, \Delta E_{0}, t_{\text {alum }}\right) \int d\left(\Delta E_{0}^{\prime}\right) I_{\text {ext }}\left(E_{0}^{\prime}, \Delta E_{0}^{\prime}, t_{L H_{2}}\right) \\
\int d\left(\Delta E_{1}\right) I_{\text {int }}\left(E_{1}, \Delta E_{1}\right) \int d\left(\Delta E_{3}\right) I_{\text {int }}\left(E_{3}, \Delta E_{3}\right) \int d\left(\Delta E_{4}\right) I_{\text {ext }}\left(E_{4}, \Delta E_{4}, t_{\text {alum }}\right) \\
\int d x \int d Q^{2} \int \frac{d \phi}{2 \pi}\left[\Theta\left(r_{\text {val }}-r_{\text {cut }}\right)\left(\frac{d^{2} \sigma}{d x d Q^{2}}\right)_{(\text {Born })}\left(E_{2}, x, Q^{2}\right)\right]
\end{array}
$$

where

$$
\begin{aligned}
& E_{0}^{\prime}=E_{0}-\Delta E_{0} \\
& E_{1}=E_{0}^{\prime}-\Delta E_{0}^{\prime} \\
& E_{2}=E_{1}-\Delta E_{1} \\
& E_{3}=E_{3}\left(E_{2}, x, Q^{2}\right) \\
& E_{4}=E_{3}-\Delta E_{3} \\
& E_{5}=E_{4}-\Delta E_{4}
\end{aligned}
$$

The integral is performed as a Monte Carlo simulation, sampling the integration volume by picking the $\Delta E$ according to the appropriate probability distribution $I_{i n t}$ or $I_{\text {ext }}$ as defined 
in sections 4.1.4.3 and 4.1.4.2. The integral is approximated by the integration volume $\Delta x \Delta Q^{2}$, multiplied by the average value of the integrand, sampled at $N_{\text {gen }}$ points:

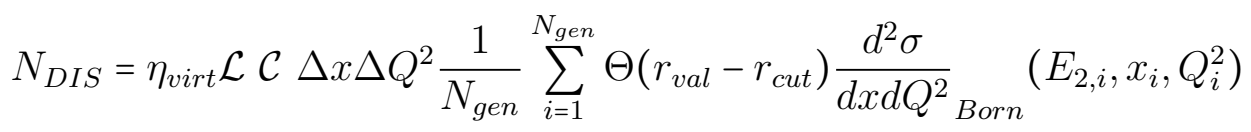

Here we can define the correct acceptance term as

$$
\Gamma_{D I S}=\Delta x \Delta Q^{2} \frac{N_{a c c}}{N_{g e n}}
$$

where $N_{a c c}$ is the number of accepted events passing all data cuts. Equation 4.33 can be rewritten as

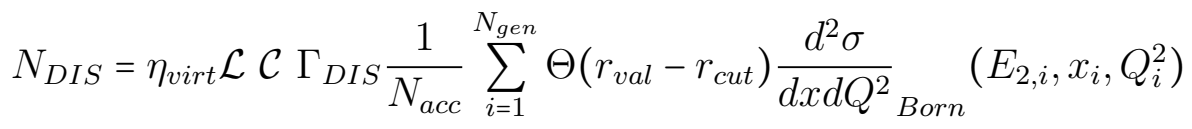

The normalized DIS cross section we wish to extract, from equation 4.1, $\frac{d^{2} \sigma}{d x d Q^{2}} H R S$, is the cross section for the nominal HRS kinematics. Because it is constant, we can factor this term outside of the sum:

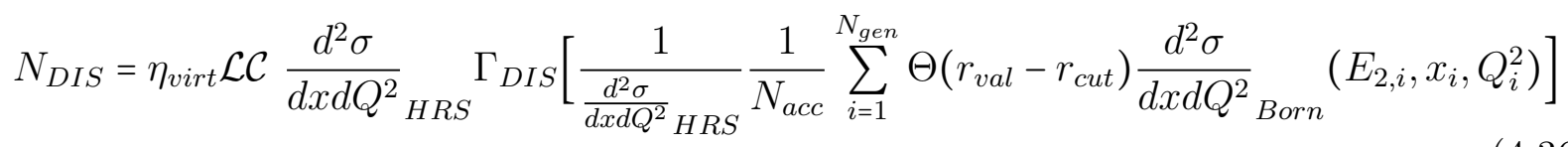

This enables us to define the variable $\alpha$ as:

$$
\alpha=\frac{1}{\frac{d^{2} \sigma}{d x d Q^{2}}(\text { HRS })} \frac{1}{N_{a c c}} \sum_{i=1}^{N_{\text {gen }}} \Theta\left(r_{\text {val }}-r_{c u t}\right) \frac{d^{2} \sigma}{d x d Q^{2}}{ }_{B o r n}\left(E_{2, i}, x_{i}, Q_{i}^{2}\right)
$$

So Equation 4.36 can be written to match the form of equation 4.1 .

$$
N_{D I S}=\mathcal{L} C \frac{d^{2} \sigma}{d x d Q^{2}} \Gamma_{D I S S)} \alpha
$$

In this formulation, the DIS parameterization of Christy and Bosted [60] is used with the Monte Carlo data to find $\alpha$ and $\Gamma_{D I S}$ for every kinematic setting. These are the corrections that account for events which have moved into or out of the HRS acceptance due to radiative effects. Table 4.7 below contains the results of calculating these terms. 


\begin{tabular}{|c|c|c|}
\hline \multicolumn{3}{|c|}{ Important DIS Cross Section Parameters } \\
\hline Setting & $\alpha$ & $\Gamma_{D I S}\left[\mathrm{GeV}^{2}\right]$ \\
\hline 361 & 0.948 & $0.503 \times 10^{-3}$ \\
362 & 0.867 & $0.726 \times 10^{-3}$ \\
363 & 0.862 & $1.143 \times 10^{-3}$ \\
481 & 0.946 & $0.116 \times 10^{-3}$ \\
482 & 1.226 & $0.508 \times 10^{-3}$ \\
483 & 1.057 & $0.422 \times 10^{-3}$ \\
484 & 1.133 & $0.443 \times 10^{-3}$ \\
601 & 0.914 & $1.405 \times 10^{-3}$ \\
603 & 0.920 & $0.904 \times 10^{-3}$ \\
\hline
\end{tabular}

Table 4.7: Results of calculating $\alpha$ and $\Gamma_{D I S}$ for all kinematic settings

\subsection{Results}

The DIS cross section was extracted for every run of each kinematic setting. The average extracted cross section is listed in Table 4.8. The experimental result is compared to the reference cross section, found using the Christy/Bosted fit [60]. The measured results for all kinematics besides 481 and 361 are unexpectedly low compared to the reference cross section, which is expected to have an error of $2 \%$ 60. In this dissertation, the DVCS cross section was extracted for the Kin-48 settings. The reason for the large discrepancy in measured vs. reference cross section for these settings is not known. It is therefore not understood if the extracted DVCS cross sections would be effected. This is visited in section 6.3.2.

\begin{tabular}{|c|c|c|c|}
\hline \multicolumn{4}{|c|}{ DIS Cross Sections (Reference and Extracted) } \\
\hline Setting & $\frac{d^{2} \sigma}{d x d Q^{2}} \operatorname{Ref}\left[\mathrm{GeV}^{-2}\right]$ & $\frac{d^{2} \sigma}{d x d Q^{2}} \operatorname{Exp}\left[\mathrm{GeV}^{-2}\right]$ & Percent Difference \\
\hline 361 & $27.98 \times 10^{-6}$ & $28.69 \times 10^{-6}$ & $+1 \%$ \\
362 & $20.45 \times 10^{-6}$ & $19.43 \times 10^{-6}$ & $-6 \%$ \\
363 & $13.18 \times 10^{-6}$ & $12.39 \times 10^{-6}$ & $-6 \%$ \\
481 & $19.54 \times 10^{-6}$ & $19.54 \times 10^{-6}$ & $0 \%$ \\
482 & $7.61 \times 10^{-6}$ & $7.15 \times 10^{-6}$ & $-6 \%$ \\
483 & $4.57 \times 10^{-6}$ & $4.16 \times 10^{-6}$ & $-9 \%$ \\
484 & $2.53 \times 10^{-6}$ & $2.30 \times 10^{-6}$ & $-9 \%$ \\
601 & $2.05 \times 10^{-6}$ & $1.93 \times 10^{-6}$ & $-6 \%$ \\
603 & $0.70 \times 10^{-6}$ & $0.69 \times 10^{-6}$ & $-3 \%$ \\
\hline
\end{tabular}

Table 4.8: A table of extracted DIS cross section results. 


\subsubsection{Fall 2014}

The DIS cross section extracted from the Fall 2014 data is shown in Figure 4.17. The black dashed line represents the reference cross section for this kinematic setting (around 28 on the vertical axis). The pink dotted line (around 28.2 on the vertical axis) represents the average extracted cross section from all runs. The pink band represents the standard deviation associated with the average. The error bars are statistical only for each run.

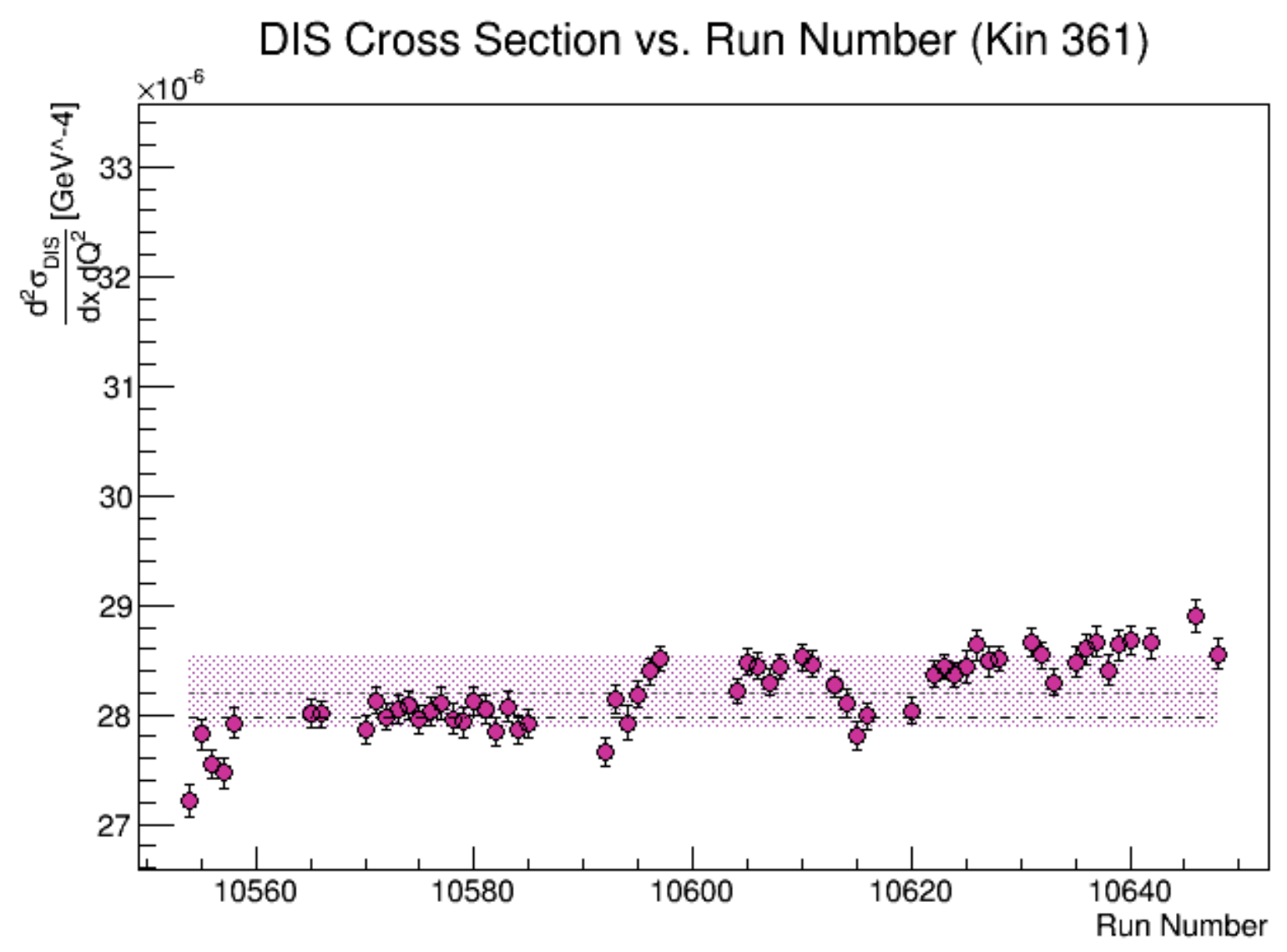

Figure 4.17: Results of extracting the DIS cross section for Kin 361.

\subsubsection{Spring 2016}

The DIS cross section extracted from the Spring 2016 data is shown in Figure 4.18. The horizontal black dot-dashed line represents the reference cross section for each kinematic setting. The horizontal grey dashed line represents the average extracted cross section from all runs for each kinematic. The grey bands represent the standard deviation associated with the average in each region. The vertical dotted lines represent regions in which the different trigger prescales changed. The error bars are statistical only for each run.

Note that the 482, 483, and 484 kinematic settings suffered from a detuned Q1 magnet, and the unknown effects of this may be related to the dramatic difference in extracted and 
reference cross section for these runs.
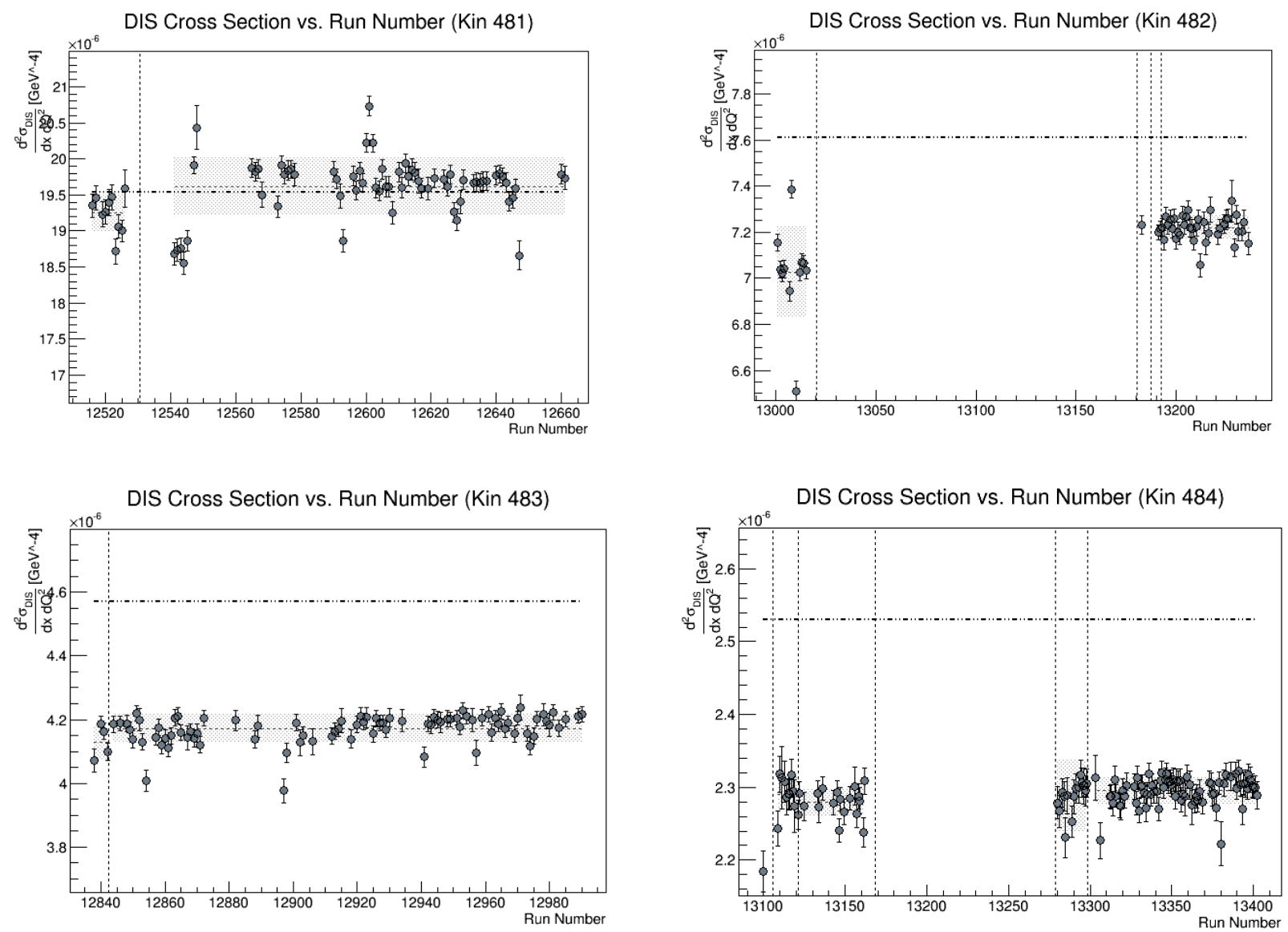

Figure 4.18: Results of extracting the DIS Cross Section for Spring 2016 Kinematics

\subsubsection{Fall 2016}

The DIS cross section extracted from the Fall 2016 data is shown in Figure 4.19. The black dot-dashed line represents the reference cross section for each kinematic setting. The grey dashed line represents the average extracted cross section from all runs for each kinematic. The blue (yellow) bands represent the standard deviation associated with the average in each region. The vertical dotted lines represent regions in which the different trigger prescales changed. The error bars are statistical only for each run.

Note that the replacement Q1 magnet from Spring 2016 suffered from an unexpected saturation, the effects of which could be related to the discrepancy between the measured and reference cross sections for these kinematics. 

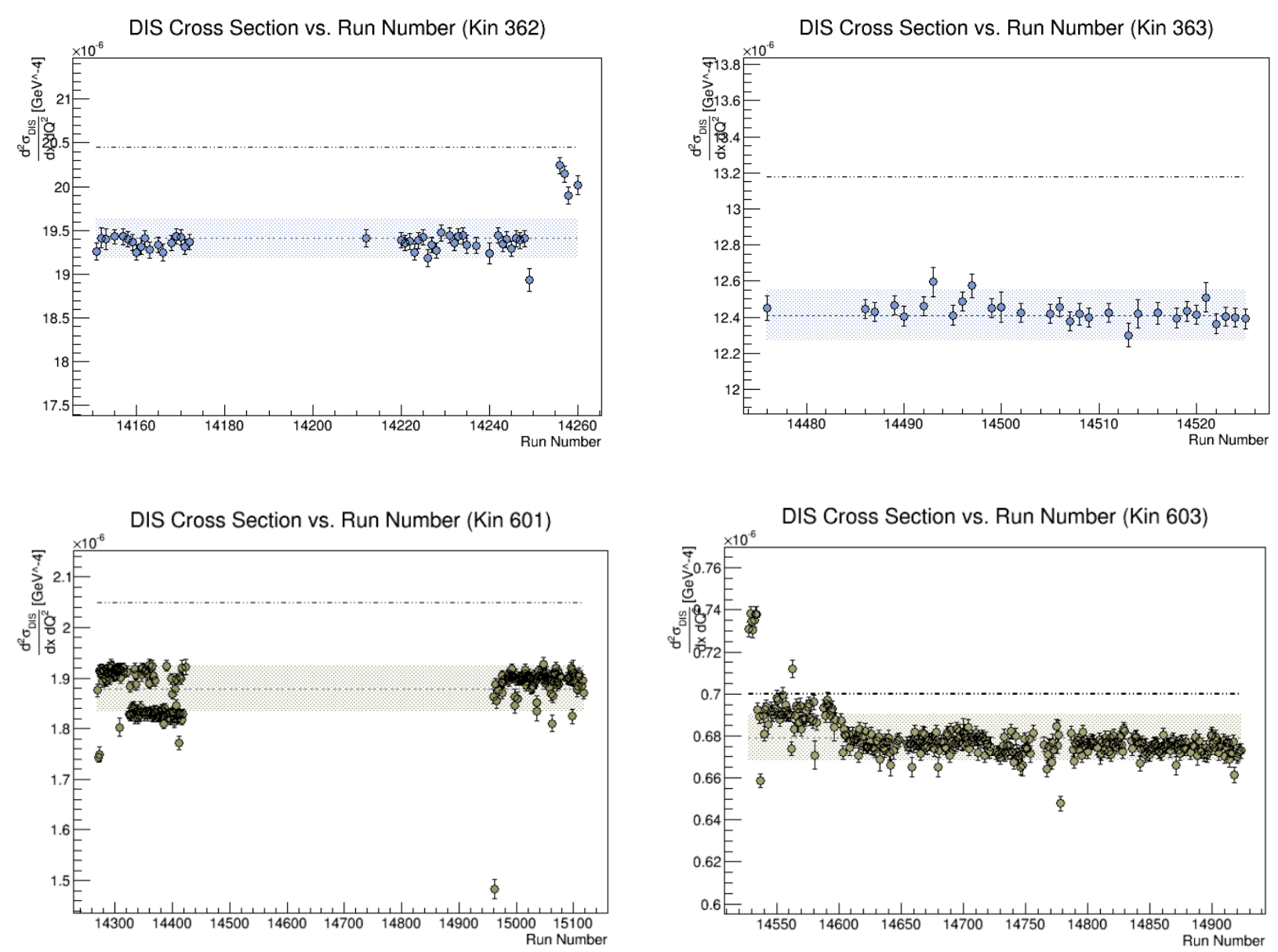

Figure 4.19: Results of extracting the DIS Cross Section for Fall 2016 Kinematics. The outlying events in Kin 362 were not found to correlate with higher current or lower tracking efficiency. The outlying set of runs in Kin 601 correlated with selecting the DIS S2M \& Cer trigger instead of the level1-DVCS S2M \& Cer trigger, whose runs did not show this discrepancy. The group of runs for Kin 603 whose measured cross section is approximately $2 \%$ larger than the rest of runs, have lower tracking efficiencies but no change in current compared to the other runs. These features were not further investigated for the sake of a timely completion of my degree. 


\section{Chapter 5}

\section{The DVCS Data Analysis}

\subsection{The Analog Ring Samplers (ARS)}

The calorimeter sits in close proximity to the beam pipe, and the scattering target. Also, E-12-06-114 is a high luminosity experiment. Because of these, high event rates are expected to be seen in the calorimeter, and pile-up of event signals is expected to be a problem. In anticipation of this, the ARS device was designed and incorporated into the DAQ, to help distinguish piled-up events in the calorimeter.

The signal from each block of the calorimeter is sampled by an ARS at a $1 \mathrm{GHz}$ rate. Within an ARS are 128 capacitors forming a circular ring buffer with each capacitor storing $1 \mathrm{~ns}$ of integrated charge. Therefore, after $128 \mathrm{~ns}$, the first capacitor will begin overwriting, and at any given time there will be a history of $128 \mathrm{~ns}$ worth of data stored in the ARS.

When the Trigger Supervisor requires a calorimeter readout to the DAQ, the ARS recording is temporarily stopped, and the data stored in the capacitor array is digitized and recorded. This process takes a long $128 \mu \mathrm{s}$, but piled up photons within the $128 \mathrm{~ns}$ of data of the ARS can be distinguished within 4 ns.

\subsubsection{Trigger system}

Because of the large deadtime associated with the ARS readout, the trigger system is designed to perform this step only if a certain threshold is reached in neighboring calorimeter blocks.

In addition to each block's signal being sampled by the ARS, a copy of the signal is also sent to an ADC which integrates the signal over the last $60 \mathrm{~ns}$. When the trigger supervisor sees a coincidence between the S2 scintillator and Cherenkov detectors in the HRS, the ARS receives the STOP signal. Instead of immediately demanding the lengthy ARS readout, the ADC signals are summed for all possible $2 \times 2$ neighboring block combinations. If any of these sums are above a pre-determined threshold, then the ARS receives a VALID signal and the $128 \mu$ s digitizing and read out process begins. 


\subsection{Waveform Analysis}

To reconstruct the dynamics of a photon hitting the calorimeter, we start by finding the timing and amplitude of the signal seen in the ARS for each block. We first assume that each pulse (signal) is a scaled version of the same shape, that appears above some baseline. The assumed shape is called the reference shape. Fitting the reference shape to the data entails scaling the reference shape's amplitude, and offsetting the pulse to the correct arrival time. The reference pulse of each block is determined during the elastic calibration of the calorimeter; it is the averaged pulse from each event of the run, and is normalized so the integrated pulse signal is one.

\subsubsection{Baseline Determination}

Determining a baseline is the first step for deciding if there is a pulse to fit. We start by finding an $80 \mathrm{~ns}$ time window $\left[i_{\text {start }}, i_{\text {start }}+79\right]$ (reduced from $128 \mathrm{~ns}$ to reduce computation time and eliminate most accidental pulses) in which the signal is flat. We vary a baseline $b$ to fit the signal $\left\{x_{i}\right\}$ by minimizing the $\chi^{2}$ :

$$
\chi^{2}=\sum_{i=i_{\text {start }}}^{i_{\text {start }}+79}\left(x_{i}-b\right)^{2}
$$

The $\chi^{2}$ minimization yields the best value of $b$ :

$$
b=\frac{1}{79} \sum_{i_{\text {start }}}^{i_{\text {start }}+79} x_{i}
$$

where $i$ represents a 1 ns sample and $i_{\text {start }}$ is chosen wisely for each separate block. Once the baseline value $b$ is established for the 80 ns window, we look in a smaller $40 \mathrm{~ns}$ window to test for a pulse. The 40 ns time window $\left[j_{\text {start }}, j_{\text {start }}+39\right]$ is centered about when we expect a pulse to arrive, taken from the time of the reference pulse. We calculate $\chi_{\text {test }}^{2}$ for the new time window:

$$
\chi_{\text {test }}^{2}=\sum_{j=j_{\text {start }}}^{j_{\text {start }}+39}\left(x_{j}-b\right)^{2}
$$

The calculated $\chi_{\text {test }}^{2}$ value is then compared to a threshold value $\chi_{0}^{2}$. If $\chi_{\text {test }}^{2}>\chi_{0}^{2}$ then a pulse is present in the channel and will be fit.

\subsubsection{Single-pulse fit}

Fitting a single-pulse event is similar to fitting the baseline, only now we need the reference pulse signal in each bin, $h_{i-t}$, the reference pulse scaling parameter $a$, and a timing bin offset $t$. The bin offset $t$ accounts for the time offset between the actual ARS signal and the reference pulse, and hence the $i-t$ subscript on $h$. For a given 80 ns time window of data 
$\left\{x_{i}\right\}$, we have a baseline $b$ and a pulse with amplitude $a$. To find $a$ and $b$, we again minimize $\chi^{2}(t)$ :

$$
\chi^{2}(t)=\sum_{i=i_{\text {start }}}^{i_{\text {start }}+80}\left(x_{i}-a h_{i-t}-b\right)^{2}
$$

The partial derivatives in $a$ and $b$ yield a set of linear equations, solvable for $a$ and $b$ for any given arrival time. Next, $\chi^{2}(t)$ is found for every $t$ in the window [-20, 25] ns. The optimal $t$ is the choice that minimizes $\chi^{2}$, and is the $t$ used when comparing $\chi^{2}(t)$ to a threshold $\chi_{1}^{2}$. Similar to a baseline fit test, if $\chi^{2}(t)>\chi_{1}^{2}$, then a one pulse fit is not satisfactory, and a fit with two pulses is made. An example of a single fitted pulse is shown in figure 5.1 .

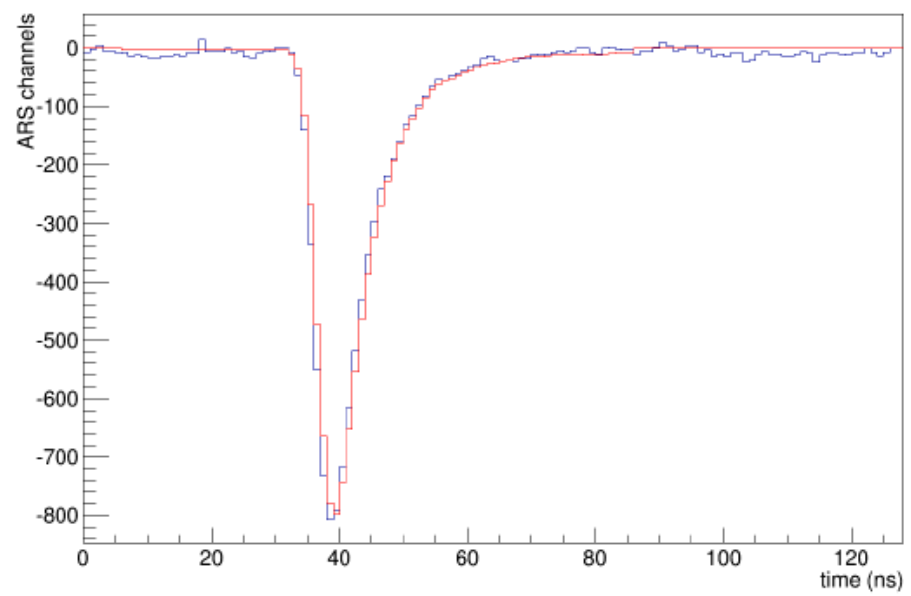

Figure 5.1: An example of a single fitted pulse over the baseline. Figure extracted from 63

\subsubsection{Two-pulse fit}

If an event fails a single-pulse fit test, we attempt a two-pulse fit. Now, we minimize $\chi^{2}$ for two pulse arrival times $t_{1}, t_{2}$, two pulse amplitudes $a_{1}, a_{2}$, and a flat baseline $b$ :

$$
\chi^{2}\left(t_{1}, t_{2}\right)=\sum_{i=i_{\text {start }}}^{i_{\text {start }}+80}\left(x_{i}-a_{1} h_{i-t_{1}}-a_{2} h_{i-t_{2}}-b\right)^{2}
$$

Following the process used for a single-pulse fit, we take partial derivatives with respect to $a_{1}, a_{2}$, and $b$ for a solvable set of linear equations. The values of $t_{1}$ and $t_{2}$ are varied to give multiple resulting $\chi^{2}\left(t_{1}, t_{2}\right)$. The $\left(t_{1}, t_{2}\right)$ combination that minimizes $\chi^{2}$ is the set of arrival times for the two pulses. And example of two fitted pulses in shown in figure 5.2. Pulses are resolvable with a minimum separation of $4 \mathrm{~ns}$. 


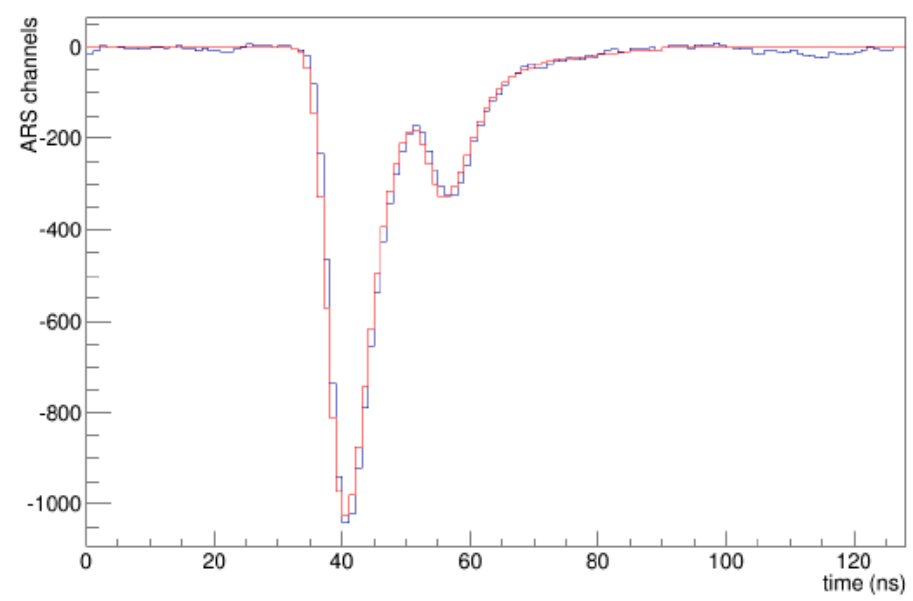

Figure 5.2: An example of two fitted pulses over the baseline. Figure extracted from [63]

Once each block's signal has been fitted for zero, one, or two pulses, the next step is to determine the energy of each pulse, and eventually cluster blocks together to reconstruct photon dynamics.

\subsection{Clustering}

After the waveform analysis is complete, we need to reconstruct the energy, time, and position for photons seen in the calorimeter. The first step is to identify which blocks are relevant for the analysis. Including all of the blocks will overestimate the photon energy due to noise, and only considering a single block will underestimate the photon energy, as some of the energy of an event will be in neighboring blocks.

\subsubsection{Assigning Blocks to Clusters}

The clustering algorithm begins by considering all 2x2 block combinations, and calculates the total energy contained in each set (if a block had two fitted pulses, the larger energy pulse is used). If the total energy of a set of 4 blocks is above the preset trigger threshold, all four blocks are kept for analysis. Besides the blocks on the edge of the calorimeter, which are only part of two $2 \mathrm{x} 2$ groups, each block is part of four $2 \mathrm{x} 2$ groups. Only a single group needs to satisfy the threshold condition once for the block to be kept. 


\begin{tabular}{|c|c|c|}
\hline \multicolumn{2}{|c|}{ Calorimeter Trigger Threshold } \\
\hline Setting & Trigger Threshold $(\mathrm{GeV})$ & Expected photon energy $(\mathrm{GeV})$ \\
\hline 361 & 3.0 & 4.6 \\
362 & 3.8 & 5.2 \\
363 & 4.8 & 6.5 \\
481 & 2.0 & 2.8 \\
482 & 3.0 & 4.6 \\
483 & 4.0 & 5.7 \\
484 & 5.8 & 7.4 \\
601 & 2.8 & 4.5 \\
603 & 5.0 & 7.0 \\
\hline
\end{tabular}

Table 5.1: A table of neighboring 2x2 block array energy thresholds for events in the calorimeter.

After each block group has been tested for energy requirements (table 5.1), the coincidence timing is considered. Events need to occur within a $[-3,+3]$ ns window relative to the HRS to be a potential DVCS photon. If the timing of a block is outside of this window, at $+13 \mathrm{~ns}$ for example, it does not pass the timing test and is thrown out (even though the remaining 3 blocks from the original $2 \times 2$ group may not be). If a block had two pulses fit, the pulse with better timing is considered for this test.

The next step is to cluster blocks together. Figure 5.3 a.) shows an example of a simple event with only one photon hitting the calorimeter. Here, four $2 \times 2$ block combinations passed the energy threshold condition. The four groups have one central block (59), and the event results in only a single cluster consisting of 6 blocks, designated numbers $42,43,44,58,59$, $60,74,75,76$. 

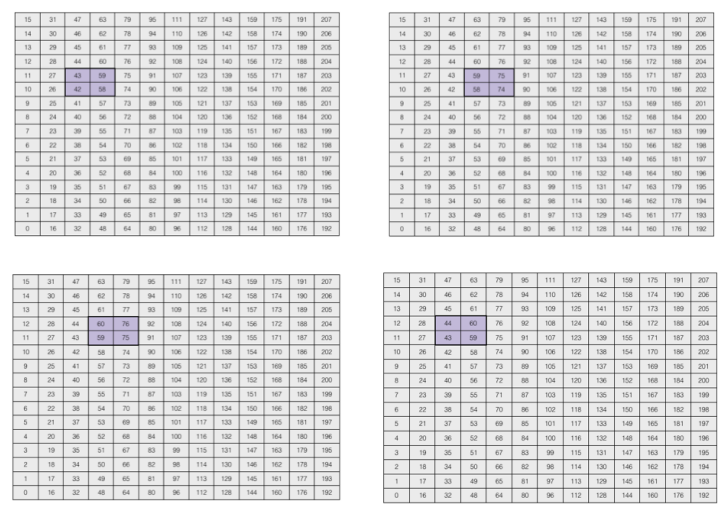

(a) The four $2 \times 2$ block groups meeting the threshold condition (labels represent block numbers). The groups are $\{42,43,58,59\}$, $\{58,59,74,75\},\{59,60,75,76\}$, and $\{43$, $44,59,60\}$.

\begin{tabular}{|l|l|l|l|l|l|l|l|l|l|l|l|l|}
\hline 15 & 31 & 47 & 63 & 79 & 95 & 111 & 127 & 143 & 159 & 175 & 191 & 207 \\
\hline 14 & 30 & 46 & 62 & 78 & 94 & 110 & 126 & 142 & 158 & 174 & 190 & 206 \\
\hline 13 & 29 & 45 & 61 & 77 & 93 & 109 & 125 & 141 & 157 & 173 & 189 & 205 \\
\hline 12 & 28 & 44 & 60 & 76 & 92 & 108 & 124 & 140 & 156 & 172 & 188 & 204 \\
\hline 11 & 27 & 43 & 59 & 75 & 91 & 107 & 123 & 139 & 155 & 171 & 187 & 203 \\
\hline 10 & 26 & 42 & 58 & 74 & 90 & 106 & 122 & 138 & 154 & 170 & 186 & 202 \\
\hline 9 & 25 & 41 & 57 & 73 & 89 & 105 & 121 & 137 & 153 & 169 & 185 & 201 \\
\hline 8 & 24 & 40 & 56 & 72 & 88 & 104 & 120 & 136 & 152 & 168 & 184 & 200 \\
\hline 7 & 23 & 39 & 55 & 71 & 87 & 103 & 119 & 135 & 151 & 167 & 183 & 199 \\
\hline 6 & 22 & 38 & 54 & 70 & 86 & 102 & 118 & 134 & 150 & 166 & 182 & 198 \\
\hline 5 & 21 & 37 & 53 & 69 & 85 & 101 & 117 & 133 & 149 & 165 & 181 & 197 \\
\hline 4 & 20 & 36 & 52 & 68 & 84 & 100 & 116 & 132 & 148 & 164 & 180 & 196 \\
\hline 3 & 19 & 35 & 51 & 67 & 83 & 99 & 115 & 131 & 147 & 163 & 179 & 195 \\
\hline 2 & 18 & 34 & 50 & 66 & 82 & 98 & 114 & 130 & 146 & 162 & 178 & 194 \\
\hline 1 & 17 & 33 & 49 & 65 & 81 & 97 & 113 & 129 & 145 & 161 & 177 & 193 \\
\hline 0 & 16 & 32 & 48 & 64 & 80 & 96 & 112 & 128 & 144 & 160 & 176 & 192 \\
\hline
\end{tabular}

(b) The resulting single-cluster event consisting of only 9 blocks, 42, 43, 44, 58, 59, 60, $74,75,76$.

Figure 5.3: An example of the clustering algorithm for a single photon event.

Events where two photons hit the calorimeter have more complex clustering, because the 2x2 block groups need to be clustered correctly. This may seem straightforward for an event where photons hit the calorimeter on opposite edges, but when the incident blocks are nearby, the situation is more complicated. Again, once all blocks passing energy and timing conditions have been identified (Figure 5.4 (1)), they are sorted into clusters. Each of the blocks is tagged with the energy it contained for the event, and the "maximal block" (the block with the largest signal, assumed to be the block where the photon actually struck) are identified (Figure $5.4(2)$ ). Next, the blocks surrounding each of the maxima get assigned to the block they surround. If a block is a neighbor to more than one maximum block, then the higher-energy block takes priority (Figure 5.4(3)). Finally, neighbors to those blocks get assigned to an original block in the same way, and the process is repeated until all accepted blocks are assigned to a cluster. 

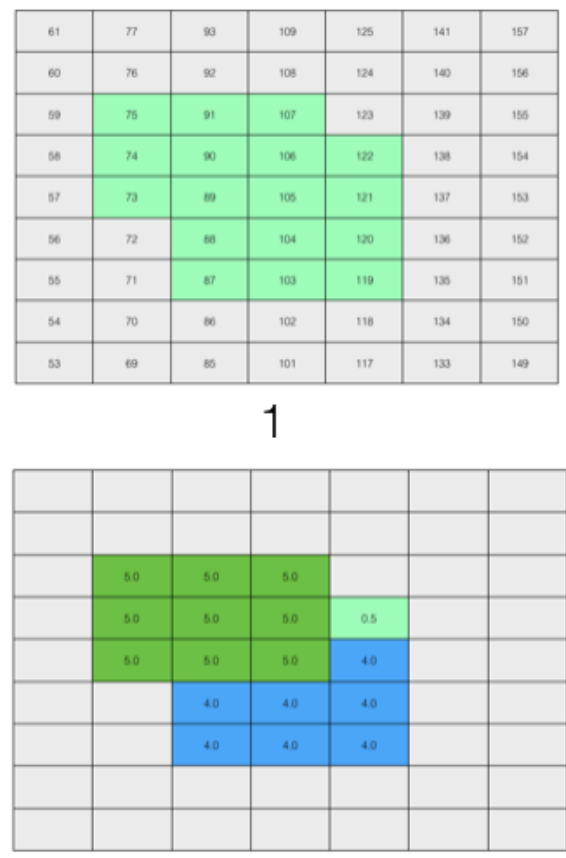

3

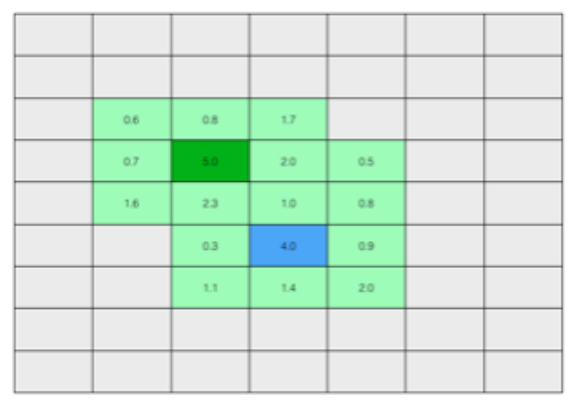

2

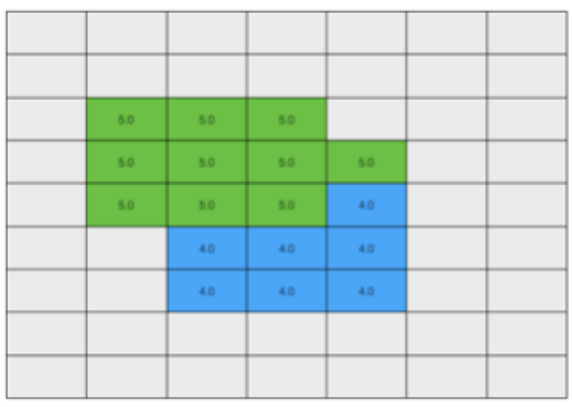

4

Figure 5.4: An example of the clustering algorithm for an event with two incident photons on nearby blocks. Image 1 shows the initial blocks passing the $2 \mathrm{x} 2$ neighbor energy test. Image 2 shows the identified blocks with local maximum energies. Image 3 shows the first step of the cluster assignment, with the higher energy block taking priority for absorbing neighbors into its cluster. Image 4 shows the final step of the clustering algorithm, with all blocks assigned to a cluster.

\subsubsection{Photon Reconstruction}

Once block clusters have been identified for each event, the photon energy, time, and position are reconstructed. For a given photon, the corresponding cluster involves $n$ blocks. Each of the blocks has deposited energy $E_{i}$ (the total photon energy $E=\sum_{i} E_{i}$ ), and position $\left(x_{i}\right.$, $\left.y_{i}\right)$.

The photon event gets assigned a position in the calorimeter by weighting the cluster blocks' positions according to the energy fractions they carry. Similarly, the time of the event is assigned by weighting the ARS times by the individual block energies. Corrections are applied to these positions that account for the block depth; that is, the shower does not always form at the block surface. Additionally, the extended target length does not guarantee that events originate at the origin, so a vertex correction is applied to produce the photon momentum vector. 


\subsubsection{Calorimeter Calibration}

In order to extract energy data from fitted wave pulses, each block of the calorimeter needs to be calibrated. There are three methods we used to do the calibration: cosmic calibration, elastic scattering calibration, and $\pi^{0}$ calibration. The first method, cosmic calibration, with the beam off, uses cosmic muons (that deposit energy of $\sim 35 \mathrm{MeV}$ ) vertically hitting the calorimeter to provide the same energy in each block. Then, the high voltage of each block's PMT is adjusted so that the resulting signal seen by each will be roughly the same. This gives a starting point for high voltage choices, and makes sure everything is wired correctly before moving to elastic and $\pi^{0}$ calibrations.

\subsubsection{Elastic Calibration}

Each block of the calorimeter was calibrated using elastic scattering ( $\left.e p \rightarrow e^{\prime} p^{\prime}\right)$. The scattered proton was detected in the HRS, and the scattered electron was seen by the calorimeter. Because the HRS allows us to measure the momentum of the scattered proton, we know the expected momentum of the electron, which can be compared to the actual signals seen from each block. For a single event we have:

$$
E=\sum_{i=0}^{207} C_{i} A_{i}
$$

Where $E$ is the expected energy of the electron $\left(E=E_{\text {beam }}+M_{p}-E_{p}\right.$ with $E_{p}$ the scattered proton energy), $C_{i}$ is the calibration coefficient of block $i$, and $A_{i}$ is the amplitude of the pulse seen in block $i$. For an elastic scattering run of $N$ total events, the $\chi^{2}$ is minimized to find $C_{i}$ :

$$
\chi^{2}=\sum_{n=1}^{N}\left(E_{n}-\sum_{i=0}^{207} C_{i} A_{i, n}\right)^{2}
$$

This process is reliable, however it takes roughly an entire day of beamtime, so cannot be performed very regularly. Another, faster, calibration process was used in addition to elastic calibration, called $\pi^{0}$ calibration. Because it could be performed every few days, it gives a better picture of gain fluctuations in the calorimeter as a function of time (as the calorimeter experiences radiation damage). The energy resolution of approximately $3 \%$ was measured from this method.

\subsubsection{2 $\quad \pi^{0}$ Calibration}

The $\pi^{0}$ calibration method is performed concurrently with normal DVCS running, meaning dedicated beam time is not required and the calibration can be performed nearly every day. The calibration method uses the $e p \rightarrow e^{\prime} p^{\prime} \pi^{0}$ reaction to study the reconstructed $\pi^{0}$ invariant mass (figure 5.5). The procedure follows that outlined in [72, and is used to apply corrections to the calibration coefficient for each block found from the results of an initial elastic calibration. 


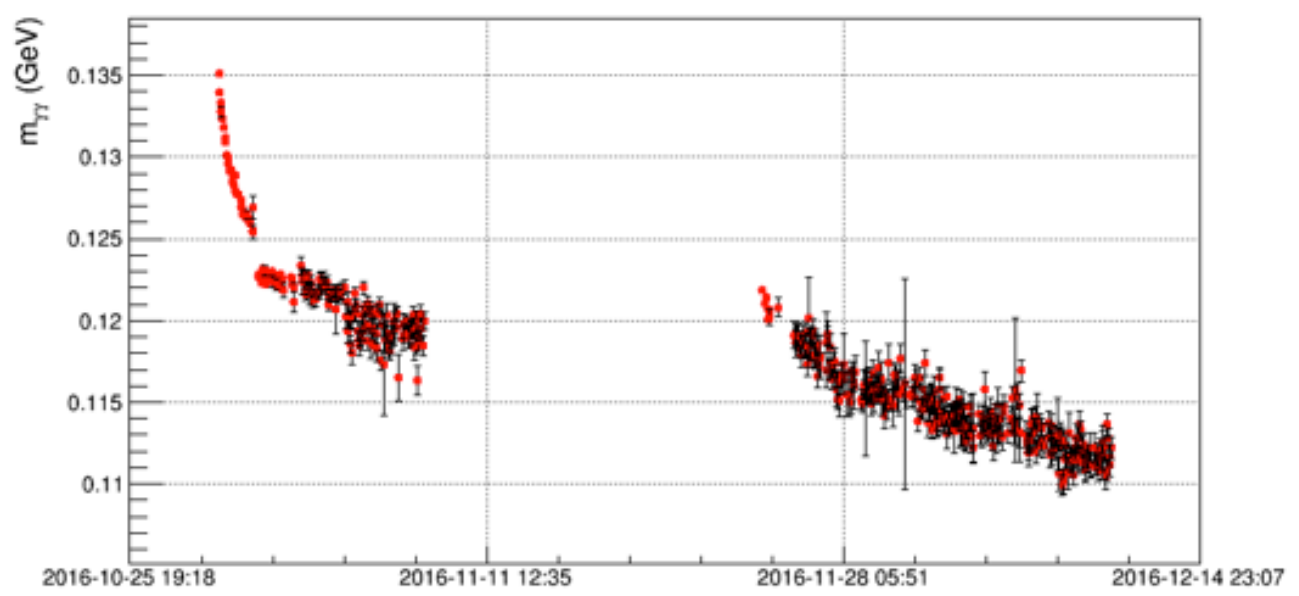

Figure 5.5: Reconstructed $\pi^{0}$ invariant mass over the duration of the Fall 2016 run period. Without calibrations of the calorimeter, the apparent $\pi^{0}$ invariant mass decreases over time due to radiation damage (darkening) in the calorimeter. Image from Dr. C. Munoz Camacho. 


\subsection{Analysis Cuts}

The analysis cuts are divided into two sections: leptonic arm and photon arm cuts. Once all of these cuts are applied, the final data set is compiled for the kinematic setting, and a missing mass cut is applied.

\subsubsection{Leptonic Arm Cuts}

The analysis cuts for the leptonic arm are the same cuts used to determine $N_{D I S}$ in Chapter 4. These include particle identification (PID), tracking, R-Function acceptance, and target cuts.

\subsubsection{Real Photon Cuts}

The exclusivity of the DVCS reaction depends on the missing mass cut that will be discussed later in Section 5.4.3. Cuts are applied to the scattered photon's position and energy (Table 5.2 in the calorimeter. Random coincidences (background) between the HRS and the calorimeter are subtracted from the data, as well as $\pi^{0}$ contamination.

\begin{tabular}{|c|c|c|}
\hline \multicolumn{3}{|c|}{ DVCS Photon Minimum Energies } \\
\hline Setting & Photon Energy Cut $(\mathrm{GeV})$ & Expected photon energy $(\mathrm{GeV})$ \\
\hline 361 & 3.0 & 4.6 \\
362 & 4.27 & 5.2 \\
363 & 4.8 & 6.5 \\
481 & 2.0 & 2.8 \\
482 & 3.0 & 4.6 \\
483 & 4.0 & 5.7 \\
484 & 5.8 & 7.4 \\
601 & 2.8 & 4.5 \\
603 & 5.0 & 7.0 \\
\hline
\end{tabular}

Table 5.2: The minimum reconstructed energy the photon must have to be included as a DVCS event, compared with the expected photon energy.

\subsubsection{Accidentals Subtraction}

True DVCS events have a scattered photon in the calorimeter within a $[-3,+3]$ ns coincidence timing window with the HRS. However, in this time window, photons can hit the calorimeter that were not produced as a result of a DVCS event. We can look outside of the true coincidence window to estimate the number of accidental events that contaminate our data. The number of accidental events should be independent of the chosen timing window, so accidentals seen in $[-21,-15] \mathrm{ns}$ or $[5,11] \mathrm{ns}$ should be the same as in $[-3,+3] \mathrm{ns}$. However, choosing any random 6 ns window will not work for the accidental subtraction, because the 
beam is delivered in $250 \mathrm{MHz}$ packets. This means an electron packet is delivered to the hall every $4 \mathrm{~ns}$, so a distribution of coincidence times for photons in the calorimeter (Figure 5.6) will have peaks at $-8 \mathrm{~ns},-4 \mathrm{~ns}, 0 \mathrm{~ns},+4 \mathrm{~ns}$, etc.. We need to choose a $6 \mathrm{~ns}$ window that is centered at one of these "peak" times, in order to accurately find accidentals.

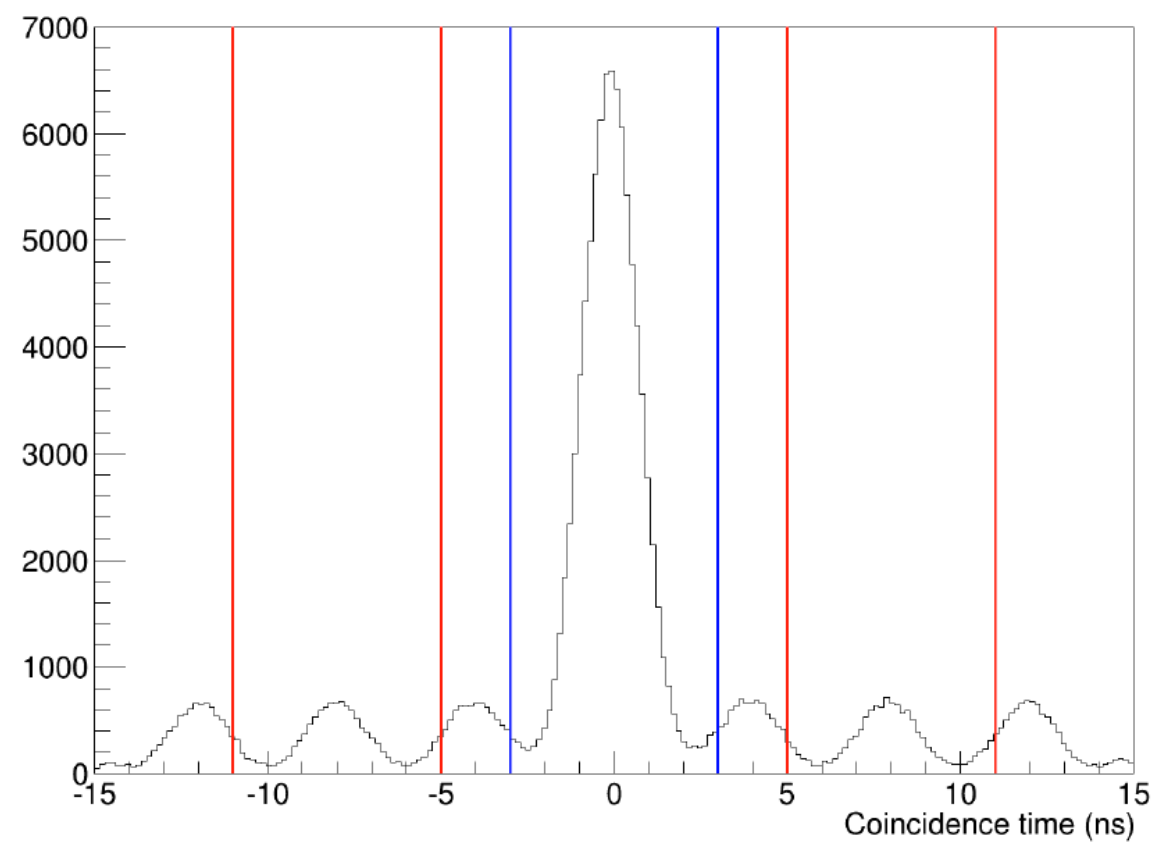

Figure 5.6: Number of events vs. coincidence time with the HRS showing the 4 ns beam packet structure.

\subsubsection{2 $\pi^{0}$ Substraction}

Events with two photons detected in the calorimeter can sometimes be the result of a $\pi^{0}$ that has decayed. While a $\pi^{0}$ will decay into two photons $99 \%$ of the time, it is possible that only one of these resulting photons will be detected in the calorimeter. In this scenario, when the detected photon passes all analysis cuts, we see a single photon in the calorimeter that appears to be a DVCS event. This set of events is called the $\pi^{0}$ contamination and is determined using a Monte Carlo simulation in combination with real $\pi^{0}$ data.

To begin, we consider all evident $\pi^{0}$ events detected in the real data. These events have two photons seen in the calorimeter that both hit non-edge blocks and pass energy threshold requirements. Additionally, we require that the event has a reconstructed mass consistent with a $\pi^{0}$. For each such event, we start our Monte Carlo simulation having the possible $\pi^{0}$ energies and momenta as a result of the $e-p$ scattering for that kinematic setting. For each of these $\pi^{0}$ events from the data, we simulate 5000 decays according to the following:

- The two photons each have energy $\frac{m_{\pi^{0}}}{2}$ in the $\pi^{0}$ center of mass (COM) frame.

- Still in the COM frame, the photons are back-to-back and have randomized direction. 
- Both photons are boosted back to the lab frame according to the $\pi^{0}$ 's momentum.

- The photons are projected to the calorimeter to determine if they are detected or not.

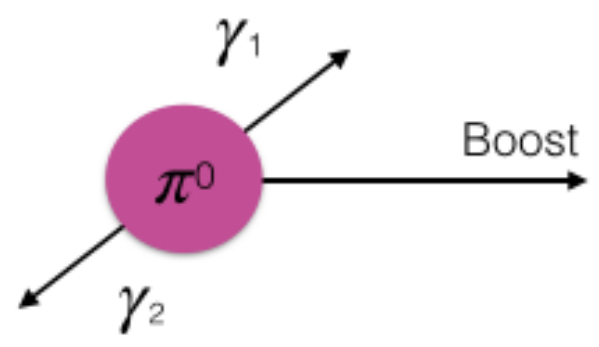

(a) $\pi^{0}$ decay for photons not perpendicular to the boost direction.

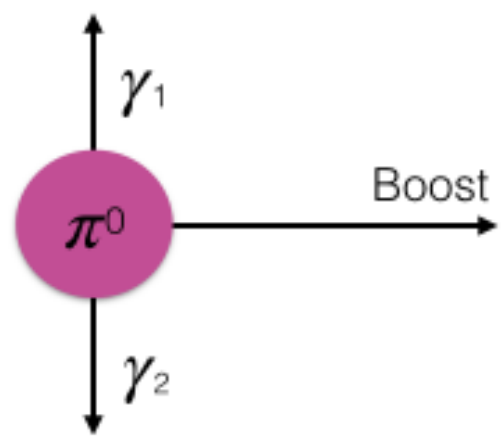

(c) $\pi^{0}$ decay for photons perpendicular to the boost direction.

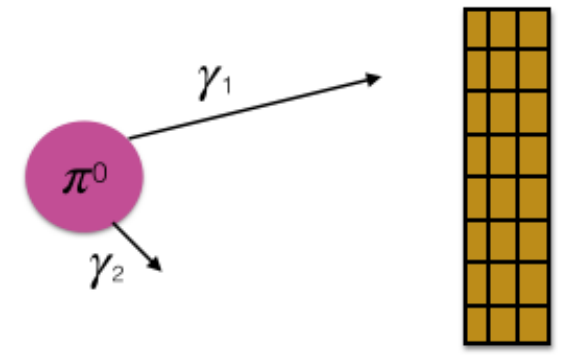

(b) Photons projected to the calorimeter for $\pi^{0}$ decay in case (a).

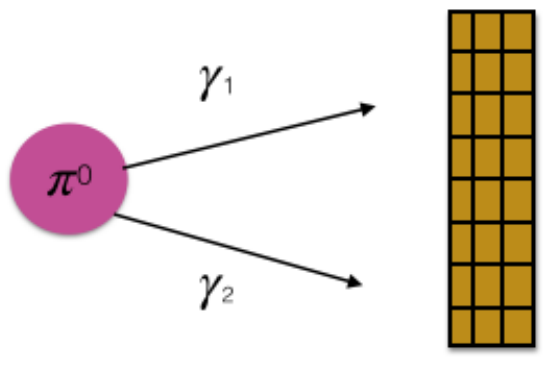

(d) Photons projected to the calorimeter for $\pi^{0}$ decay in case $(\mathrm{c})$.

Figure 5.7: $\pi^{0}$ decay in the case that photons are (c) and aren't (a) perpendicular to the boost direction.

In each of the 5000 decays, zero, one, or two of the photons will be detected in the calorimeter (and $n_{0}+n_{1}+n_{2}=5000$ ). In the case in which a single photon (above an energy threshold) is detected, we treat the event like it was a DVCS event and calculate all relevant experimental parameters (missing mass, etc.). Because the simulated single-photon events are generated from $\pi^{0}$ in real data, where both photons were detected, we need to weight the $\pi^{0}$ contamination events by $\frac{1}{5000} \frac{5000}{n_{2}}=\frac{1}{n_{2}}$ before subtracting them from DVCS data. Here, the fraction $\frac{1}{n_{2}}$ represents the probability that the given $\pi^{0}$ channel will result in a decay where a single photon is detectable in the calorimeter - thus representing the $\pi^{0}$ contamination at that event's invariant missing mass.

The effectiveness of this subtraction method was studied using a Geant4 simulation that generates $\pi^{0}$ 's. The above steps are repeated using the simulated data where two photons 
are detected in the calorimeter, to find the single photon contamination of this data set. However, because this was simulated data, we actually have a record of $\pi^{0}$ events where only a single photon hit the calorimeter. The ratio of the two is compared over the whole calorimeter to ensure an efficient subtraction method. The method described in this section was found to be efficient (ratio close to 1) with the exception of the calorimeter corners, motivating the decision to use octagonal cuts on photon position defined in Section 5.4.2.3.

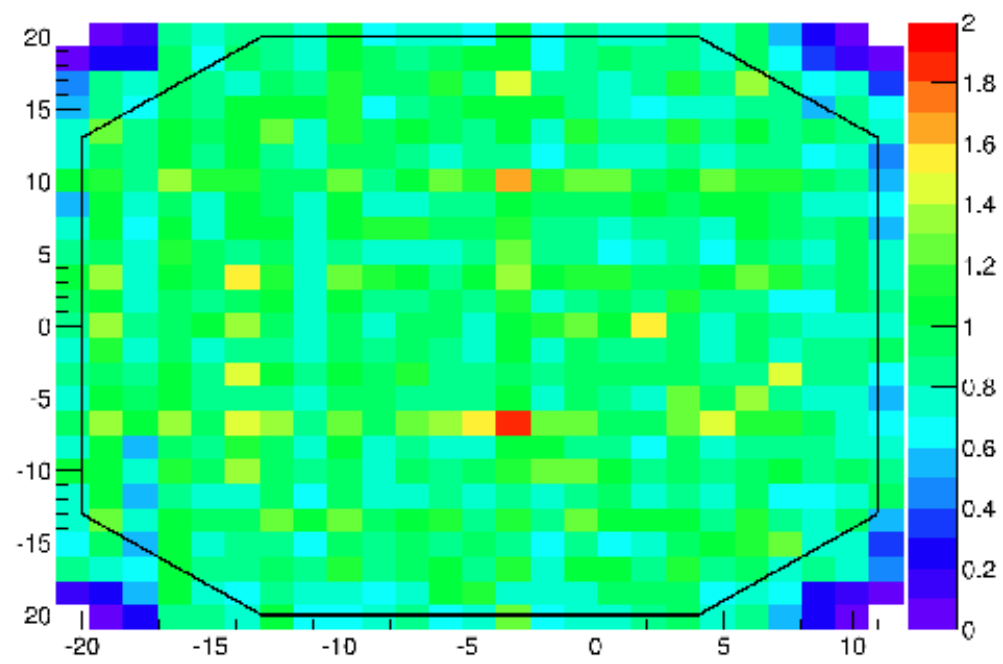

Figure 5.8: Plot from Frédéric Georges 61]. The efficiency of the $\pi^{0}$ subtraction across the calorimeter. The ratio correctly close to 1 supports the method of $\pi^{0}$ subtraction outlined in this section. The method fails to subtract the $\pi^{0}$ contamination on the corners of the calorimeter, so an octagonal cut is used for photon position. The over-subtracted regions were found to be statistical.

\subsubsection{Photon Position Cuts}

For reasons outlined in Section 5.4.2.2, cuts are applied to the corners of the calorimeter. In addition to this, cuts are applied to the edges of the calorimeter, as the photon shower cannot be contained by neighboring blocks and instead leaks out the sides. The cuts used are defined in the equations below: 


$$
\begin{aligned}
& x \leq 11 \mathrm{~cm}(7.5 \mathrm{~cm} \text { for Kin 484) } \\
& x \geq-20 \mathrm{~cm} \\
& y \leq 20 \mathrm{~cm} \\
& y \geq-20 \mathrm{~cm} \\
& y \leq x+33 \mathrm{~cm} \\
& y \leq-x+24 \mathrm{~cm} \\
& y \geq-x-33 \mathrm{~cm} \\
& y \geq x-24 \mathrm{~cm}
\end{aligned}
$$

\subsubsection{Missing Mass Cut}

The exclusivity of the DVCS reaction depends on a proton being the only detected particle in the final state. Because we don't detect this proton directly in the experimental setup, we instead perform a cut on the missing mass squared of the data, after all other analysis cuts have been applied.

The DVCS missing mass squared is defined as $M_{X}^{2}=\left(e+p-e^{\prime}-\gamma\right)^{2}$. Here, $X$ represents the final state particles, $e$ and $p$ the four vectors of the initial state electron and proton, and $e^{\prime}$ and $\gamma$ the four vectors of the final state electron and photon. For the DVCS reaction, $X$ should be a single recoil proton, and $M_{X}^{2}=M_{p}^{2} \approx 0.88 \mathrm{GeV}^{2}$. Because of resolution effects in the calorimeter, and energy uncertainties from calibration, the squared missing mass distribution is not a sharp peak. 


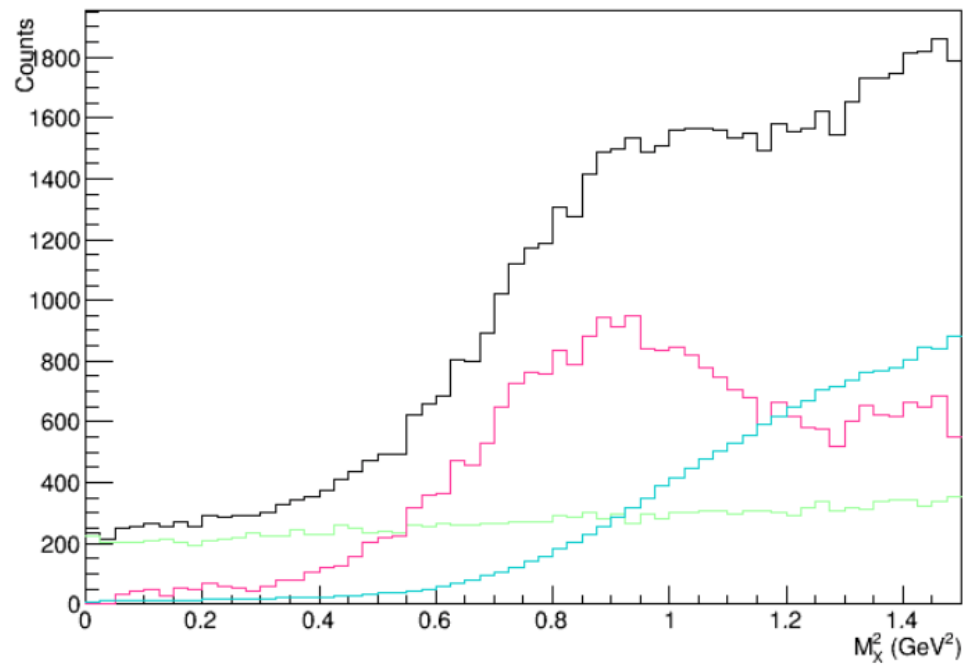

Figure 5.9: The squared missing mass distribution for kinematic 481 after all other analysis cuts have been applied. The black histogram shows accidental experimental data before any background subtraction. The green histogram shows the background events (Section 5.4.2.1), and the blue histogram is the $\pi^{0}$-contamination (Section 5.4.2.2). The pink histogram is the $M_{X}^{2}$ distribution after background-contamination and $\pi^{0}$-subtraction.

Figure 5.9 shows the missing mass squared distribution for Kin 481. A good choice of cuts on $M_{X}^{2}$ is used to ensure we have a proton (and only a proton) in the final state. The choice of cuts will be discussed more in Section 6.3.

\subsubsection{SIDIS and Resonance Contamination}

Sections 5.4.2.2 and 5.4.2.1 discussed $\pi^{0}$-contamination and background-contamination of experimental data. However, more contamination exists that is primarily removed via the missing mass cut.

\subsection{SIDIS}

Semi-Inclusive DIS (SIDIS) events that can contaminate our data of the form $e p \rightarrow e^{\prime} p^{\prime} X$. Because we don't directly detect $p^{\prime}$, SIDIS events can appear to be DVCS events, but have extra particles in the final state that we don't see. We consider the worst case scenario: the lowest possible missing mass squared for the SIDIS reaction $e p \rightarrow e^{\prime} p^{\prime} \gamma X$. A $\pi$ in the final state yields a $M_{X}^{2}$ (which includes $p^{\prime}$ ) of $\approx 1.15 \mathrm{GeV}^{2}$. While this is much larger than $M_{p}^{2} \approx 0.88 \mathrm{GeV}^{2}$, the resolution effects that broaden the $M_{X}^{2}$ distribution for DVCS will also broaden the SIDIS distribution. This means that even with a good choice of $M_{X}^{2}$ cuts, some SIDIS contamination may remain. This will be discussed more in Section 6.3. 


\subsection{Resonances}

Again because we do not detect the recoil proton directly, any event with $e p \rightarrow e^{\prime} \gamma X$ can contaminate our data. This includes reactions with a resonance in the final state. Like SIDIS, we consider the lowest possible $M_{X}^{2}$ resulting from contamination. The reaction ep $\rightarrow e^{\prime} \gamma \Delta(1232)$ gives a peak at $M_{X}^{2}=M_{\Delta(1232)}^{2} \approx 1.5 \mathrm{GeV}^{2}$. Resonance contributions are expected to present an even smaller contribution because of their larger $M_{X}^{2}$ values, and small cross section at our kinematic settings [62], so they will not be considered.

\subsection{Beam Helicity}

For polarized DVCS cross sections, the longitudinal beam polarization must be known. Both the Moller and Compton polarimeters measure beam polarization, with the Compton being parasitic and the Moller measureing at intervals during run time. Figure 5.10 shows the results of calculating the beam polarization for Spring 2016 using data from the Compton, with Moller measurements added for reference. The discrepancy in beam polarization measurements has not yet been understood by the Compton and Moller teams. 


\section{Beam Polarization vs Run Number}

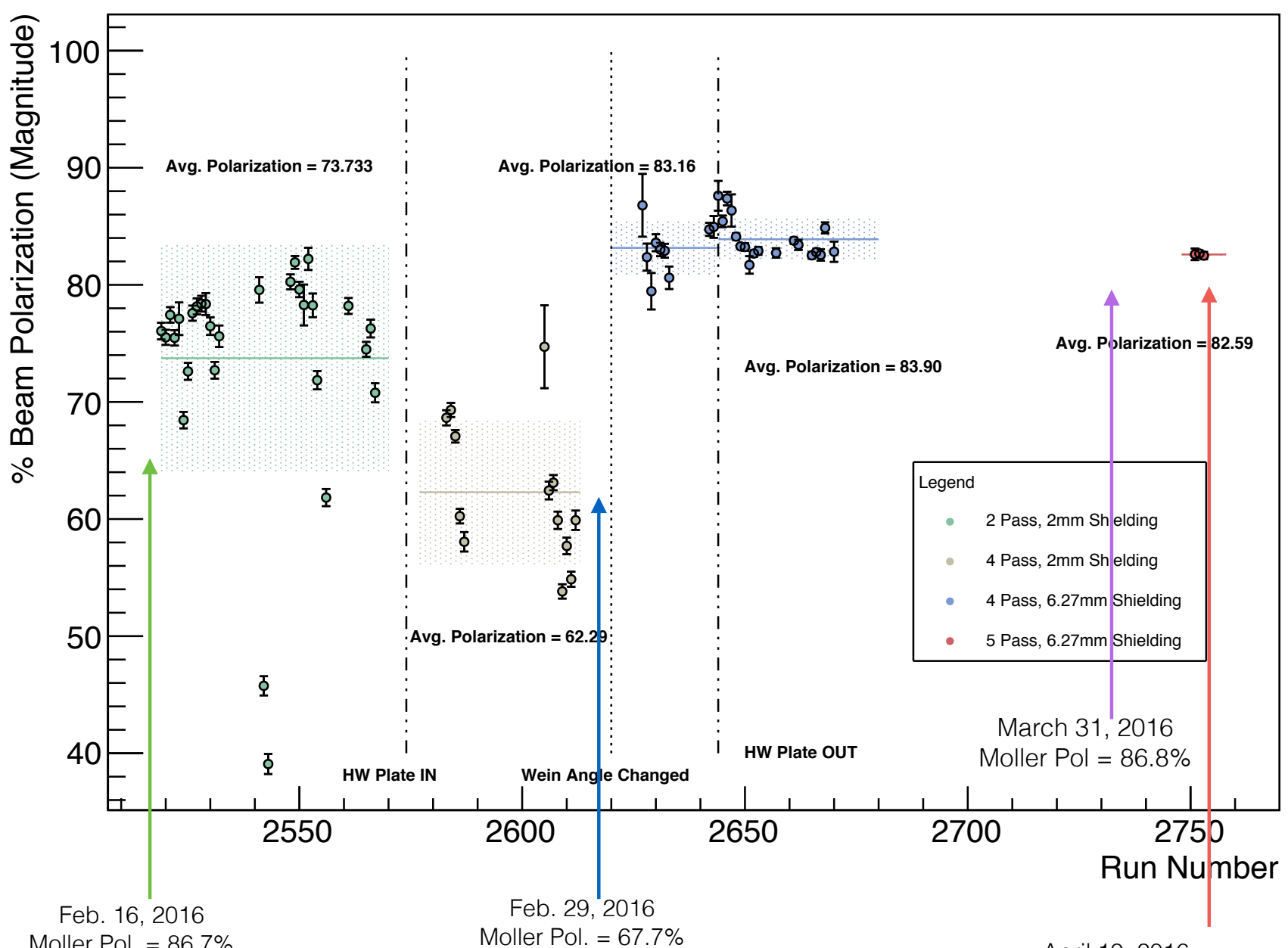

Moller Pol. $=86.7 \%$

$\longrightarrow$ Change wein angle, new Pol $=86.9 \%$

Moller Pol. $=88.1 \%$

Figure 5.10: Beam polarization measurements as calculated for the Compton for Spring 2016, with Moller measurements. The four colored arrows show when the Moller measured beam polarization, and what value was measured.

The beam polarization measurements made by the Moller were used for the DVCS analysis. 


\subsection{Geant4 Simulation}

When we extracted a DIS cross section from the data, we needed to know the phase space of the reaction. Similar to the case of DIS, we use simulation to calculate this factor for DVCS for each kinematic setting. Because of the many parameters, detector dependancies, and radiative effects, this cannot be done analytically and must be computed using simulation. This section will describe the Geant4 simulation used to find the phase space for each kinematic setting.

\subsubsection{Geometry}

The geometry of the Geant4 simulation is designed to mimic the real experimental setup, including all distances and materials (Figure 5.11). Included in the geometry is the beam pipe, all physical shielding used in the hall, the target chamber, and the calorimeter itself.

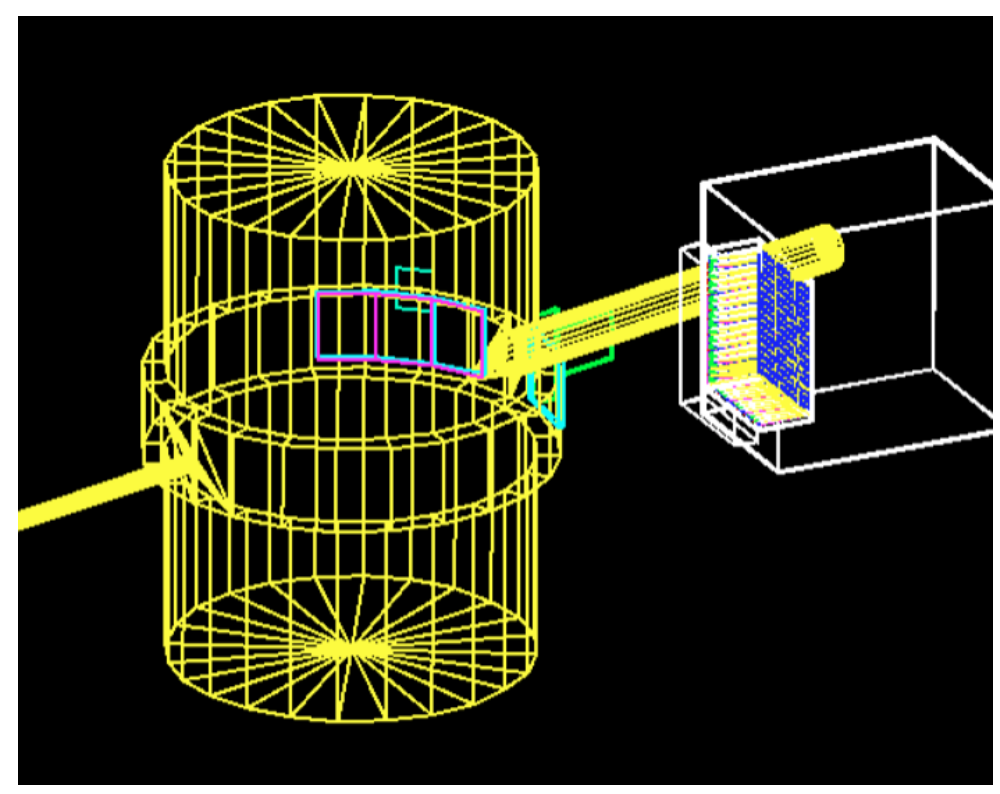

Figure 5.11: The geometry of the DVCS Geant4 simulation.

The target chamber includes the $\mathrm{LH}_{2}$ scattering target, the kapton window opening to the HRS entrance (the HRS itself is not simulated, but the R-Function can still be used to determine an event's acceptance), and the aluminum window to the calorimeter. The aluminum window opens to the calorimeter, placed the correct distance and angle for each kinematic setting. Each of the $\mathrm{PbF}_{2}$ blocks is correctly positioned within the calorimeter, and the showers of each block are handled by Geant4. 


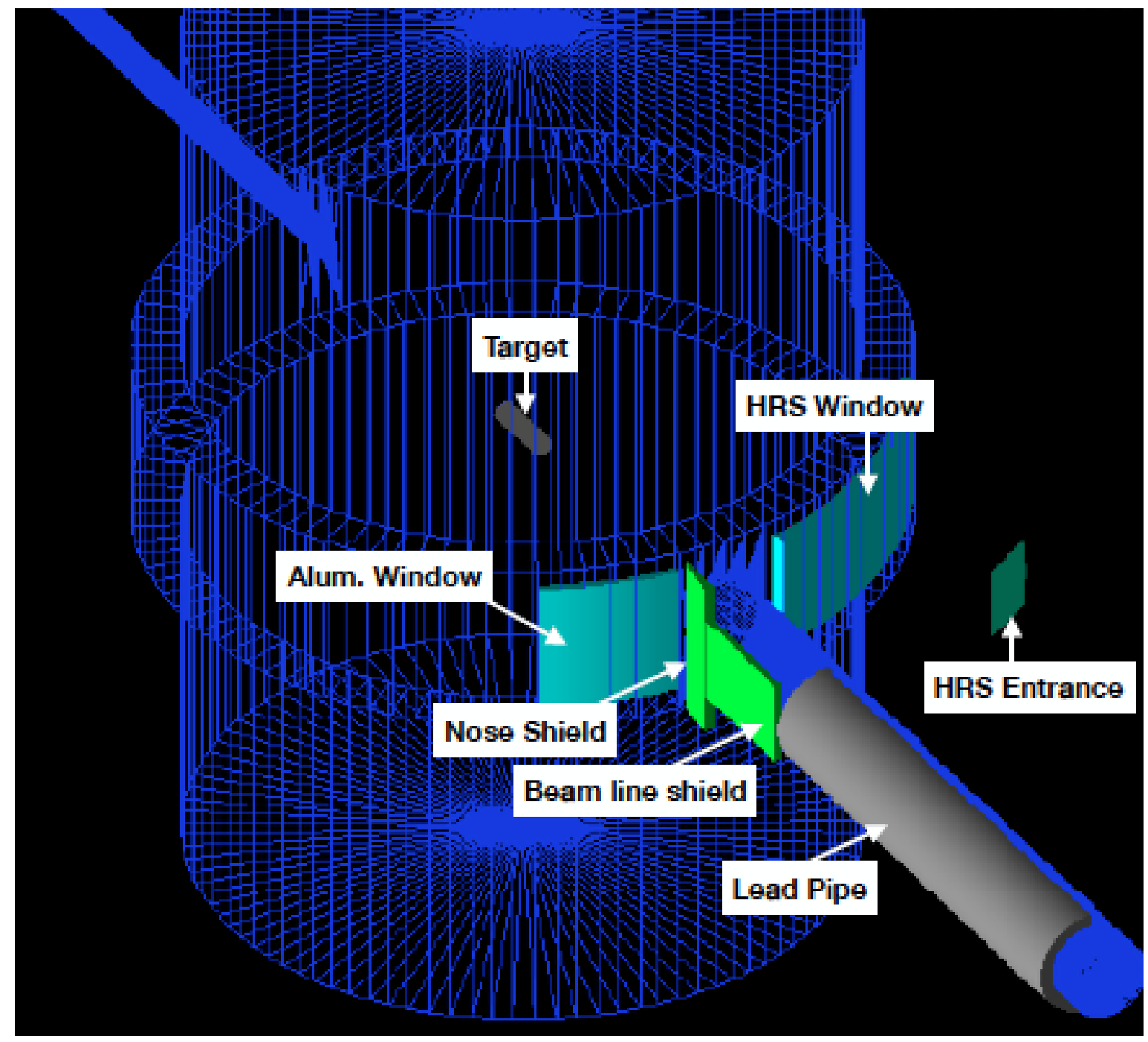

Figure 5.12: The simulated geometry of the DVCS scattering chamber. The beam travels from the top left of the page to the bottom right.

\subsubsection{Event Generator}

The DVCS reaction can be decomposed into a leptonic reaction $\left(e \rightarrow e^{\prime} \gamma^{*}\right)$ and a hadronic reaction $\left(\gamma^{*} p \rightarrow \gamma p^{\prime}\right)$. The simulation first considers the leptonic reaction, generating events in a very similar way to that described in Section 4.1.4.1, beginning by generating a scattering vertex $v_{z}$, along with the kinematic variables $x_{B}$, and $Q^{2}$.

The scattering vertex $v_{z}$ allows us to find the distance the electron traveled in the $\mathrm{LH}_{2}$, letting us calculate the electron energy after an external radiative correction, $E_{v}^{e x t}$, according to the method described in Section 4.1.4.2. In the DIS simulation, $x_{B}$ and $Q^{2}$ were generated randomly within the same bounds for each event; for DVCS it is advantageous for reducing simulation run time to restrict the bounds on $x_{B}$ and $Q^{2}$ according to $E_{v}^{e x t}$ and $\theta_{e}, p_{e}$ ranges 
nominal to HRS acceptance (the electron's horizontal scattering angle (spherical coordinates) and momentum):

$$
\begin{gathered}
4 p_{e}^{\text {min }} E_{v}^{e x t} \sin ^{2}\left(\frac{\theta_{e}^{\text {min }}}{2}\right)<Q^{2}<4 p_{e}^{\text {max }} E_{v}^{e x t} \sin ^{2}\left(\frac{\theta_{e}^{\max }}{2}\right) \\
x_{B}^{\text {min }}=\operatorname{Max}\left[\frac{Q^{2}}{2 M\left(E_{v}^{e x t}-p_{e}^{\text {min }}\right)}, 0.05\right]<x_{B}<\operatorname{Min}\left[\frac{Q^{2}}{2 M\left(E_{v}^{e x t}-p_{e}^{\text {max }}\right)}, 0.95\right]=x_{B}^{\text {max }}
\end{gathered}
$$

with $M$ the proton mass.

After $x_{B}$ and $Q^{2}$ are selected, the event must be tested to confirm it is physically possible. In the case that either $x_{B}^{\min }>x_{B}^{\max }$ or $p_{e}^{\min }>E_{v}^{e x t}$, the event is thrown out. If the event passes the test, the internal Bremsstrahlung is calculated according to section 4.1.4.3, and the electron energy after this step is labeled $E_{v}$. The kinematics for the electron after the $e \rightarrow e^{\prime} \gamma^{*}$ reaction are found according to:

$$
\begin{gathered}
p_{e}=E_{v}-\nu \\
\cos \theta_{e}=1-\frac{Q^{2}}{2 p_{e} E_{v}}
\end{gathered}
$$

where $\nu=\frac{Q^{2}}{2 M x_{B}}$. A second test that the event is physical must now be conducted, testing that $p_{e}>0$. Again, if this test fails, the event is discarded. The angle $\phi_{e}$ (spherical coordinates) is generated to cover the entire vertical range of the HRS entrance. Events are transported to this entrance after a second internal radiative correction is applied, where the R-Function can later be used in the analysis for leptonic arm cuts. 


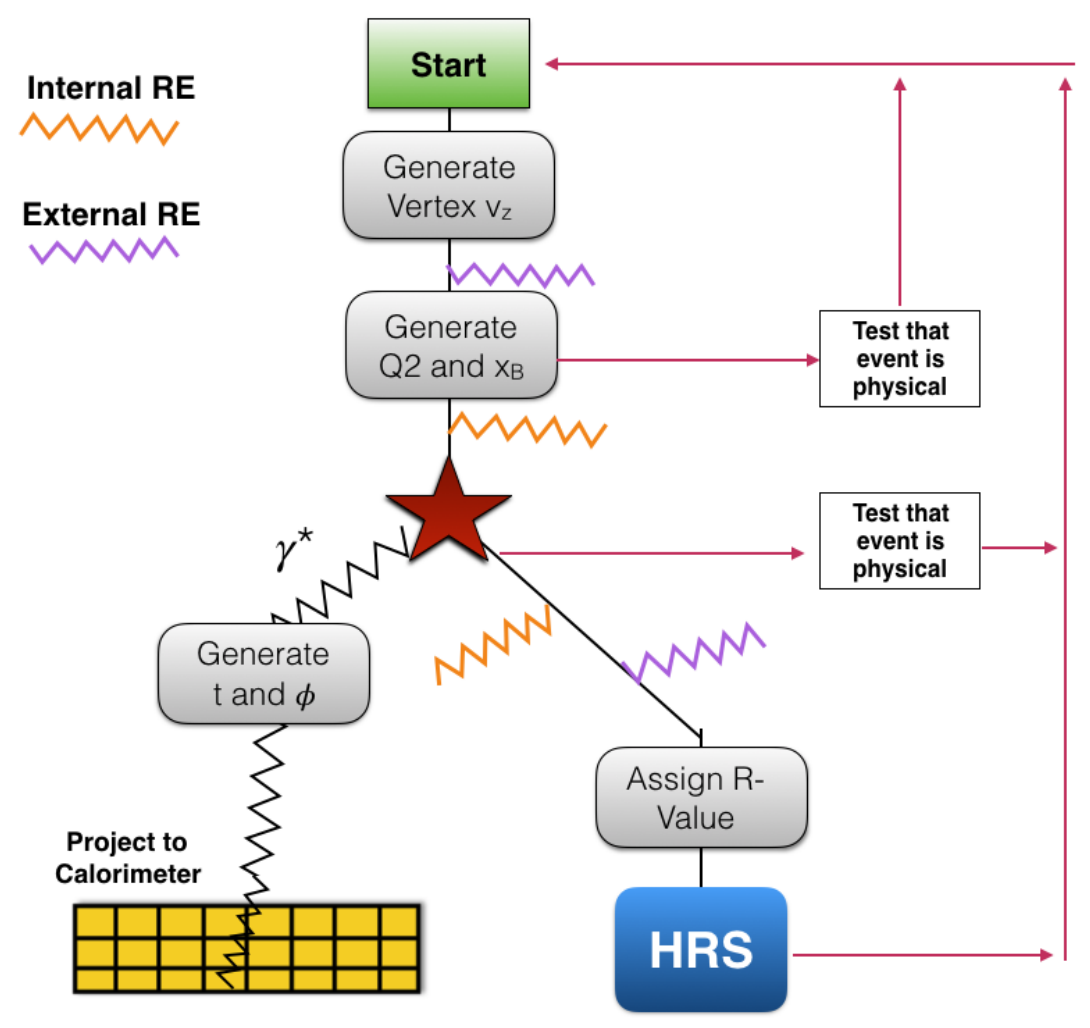

Figure 5.13: Geant4 simulation steps diagram.

The hadronic scattering $\gamma^{*} p \rightarrow p^{\prime} \gamma$ is computed next, first in the center of mass frame. The $\gamma^{*}$ momentum depends on $E_{v}$ and $p_{e}$, the electron energy before and after scattering. After calculating this quantity, the squared momentum transfer to the proton $t$ is generated between:

$$
t_{\text {min }}-2 \mathrm{GeV}^{2}<t<t_{\text {min }}
$$

with

$$
t_{\text {min }}\left(x_{B}, Q^{2}\right)=-Q^{2} \frac{2\left(1-x_{B}\right)\left(1-\sqrt{1+\epsilon^{2}}\right)+\epsilon^{2}}{4 x_{B}\left(1-x_{B}\right)+\epsilon^{2}}
$$

where an event with $t$ greater than $t_{\min }$ would be unphysical, and $\epsilon=2 x_{B} \frac{M}{Q}$, in order to cover the full acceptance space of the calorimeter (which varies between $0.4 \mathrm{GeV}^{2}$ and 1.2 $\mathrm{GeV}^{2}$ in $t$ ). The outgoing photon and proton momenta are calculated and boosted back to the lab frame. The angle between the leptonic and hadronic planes, $\phi_{\gamma \gamma}$ is then generated between $[0,2 \pi]$ and all particle momenta are rotated accordingly.

Finally, each event $i$ is assigned a phase space factor according to:

$$
\Delta \Phi_{i}=\Delta Q_{i}^{2} \Delta x_{B, i} \Delta t_{i} \Delta \phi_{\gamma \gamma, i} \Delta \phi_{e, i}
$$


Because the phase space differs for every event, the overall acceptance for generated events can be found according to

$$
\Delta \Phi=\frac{1}{N_{\text {gen }}} \sum_{i=0}^{N_{a c c}} \Delta \Phi_{i}
$$

where $N_{\text {gen }}$ is the total number of generated events, $N_{a c c}$ is the number of these events passing all analysis cuts, and $\Delta \Phi_{i}$ is the phase space factor of accepted event $i$. The overall phase space factor can also be found on an experimental bin-by-bin basis, which will be required for the DVCS cross section extraction.

\subsubsection{Simulation Smearing/Calibration}

The simulation plays an important role in determining the missing mass cut to be applied to experimental data, and the respective error it induces on the cross section measurement. Figure 5.9 showed the missing mass distribution for kinematic 481 after all analysis cuts were applied and background $+\pi^{0}$ contamination was removed. A cut on the DVCS missing mass will eliminate much of the contamination, but some true DVCS events will be lost from the radiative tail. It is important to know for a given missing mass cut, what fraction of experimental DVCS events we remove in this process. To find this, we use the simulated DVCS data from the Geant4 simulation, but first, this must be calibrated and take into account:

- Energy Resolution Differences: The energy resolution of the simulation is far better than experimental capability, manifesting as a much narrower missing mass peak. Calorimeter blocks in simulation do not experience radiation damage/darkening over time. Additionally, the simulation does not use Cherenkov photons to calculate deposited energy; the fluctuating number of Cherenkov photons experimentally is largely responsible for energy resolution. Taking these factors into account is referred to as the "simulation smearing".

- Underestimation of Photon Energy: The missing mass peak is not centered on $M_{p}$ for the simulation. Possibly this is because we ignore Cherenkov effects in simulation, but more likely the full shower is not contained in each block and energy leakage is not corrected for like it is experimentally with calibration procedures. Thus the simulation must be corrected for the calibration procedures used for the actual data.

The simulation smearing and calibration corrections are accomplished simultaneously, event by event, by generating a random number following a Gaussian distribution with mean $\mu$, and standard deviation $\frac{\sigma}{\sqrt{E}}$. Here $\mu$ is the calibration coefficient, $\sigma$ the smearing coefficient, and $E$ photon energy. The resolution is modeled as $\frac{\sigma}{\sqrt{E}}$ where $E$ is measured in $\mathrm{GeV}$ and the constant $\sigma$ determined by the fit to experimental data.

$$
\operatorname{rand}=\operatorname{Gaus}\left(\mu, \frac{\sigma}{\sqrt{E}}\right)
$$


We transform the photon energy according to:

$$
E \rightarrow \operatorname{rand} \times E
$$

The calorimeter blocks darken over time, so we find $\mu$ and $\sigma$ separately for each kinematic setting. Additionally, the blocks darken at different rates, so we decompose the calorimeter into 49 regions and find $\mu$ and $\sigma$ for each. We use the following local smearing/calibration procedure described in section 5.6.3.1.

\subsubsection{Local Smearing Procedure}

We start with the choice to decompose the calorimeter into 49 regions. The regions overlap by $50 \%$ in both $x_{c}$ and $y_{c}$ in accordance with the hexagonal spatial cuts on photon position given in Equations 5.8 through 5.14. Each has a height of $10 \mathrm{~cm}$ and a width of $7.5 \mathrm{~cm}$. The following steps are completed for all 49 regions individually to find the $\mu$ and $\sigma$ for each that result in the best fit of the missing mass distribution between the simulated and experimental data:

1. Choose $\mu \in[1.0,1.1]$ and $\sigma \in[0.05,0.5]$. These are chosen independently of each other, and each has 1000 steps. This means there are 1,000,000 possible combinations of $\mu$ and $\sigma$ that will be tested for each calorimeter region.

2. Generate a random number from a Gaussian distribution, $\operatorname{Gaus}\left(\mu, \frac{\sigma}{\sqrt{E}}\right)$, for a single event. Use this according to Equation 5.24. Repeat this step with the same $\mu$ and $\sigma$ for every event of the simulation.

3. Weight events by $\frac{B H \cdot p s f}{N_{g e n}}$, with $B H$ the Bethe-Heitler, $p s f$ the phase space factor, and $N_{\text {gen }}$ the number of events generated by the simulation.

4. Calculate the new missing mass squared for each event.

5. Find $\chi^{2}$ for the current $\mu, \sigma$ combination, in comparison with the experimental data according to:

$$
\chi^{2}(\mu, \sigma)=\frac{1}{N_{b i n}-2} \sum_{i=i_{\min }}^{i_{\max }}\left(\frac{N_{i}^{\text {data }}-N_{i}^{s i m}}{\sigma_{i}^{\text {data }}}\right)^{2}
$$

Here $N_{\text {bin }}$ is the total number of histogram bins being compared, $i_{\text {min }}$ and $i_{\text {max }}$ the range of bins, $N_{i}^{\text {data }}$ is the number of experimental events in missing mass bin $i, N_{i}^{s i m}$ the number of simulated events in missing mass bin $i$, and $\sigma_{i}^{\text {data }}$ the statistical uncertainty of bin $i$ of data. 


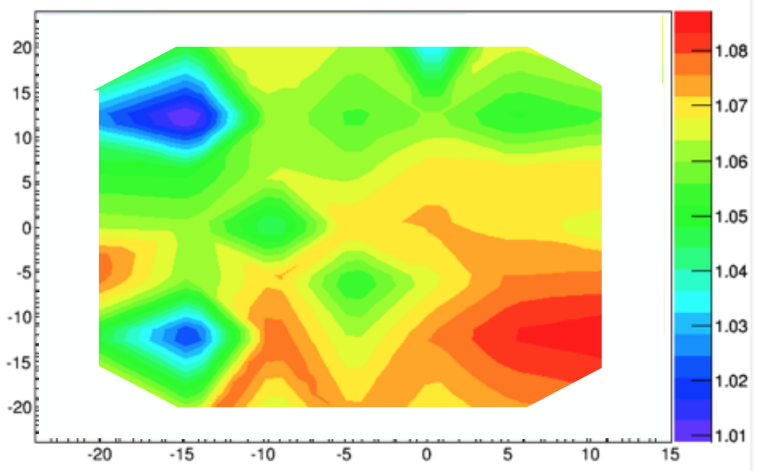

(a) $\mu$

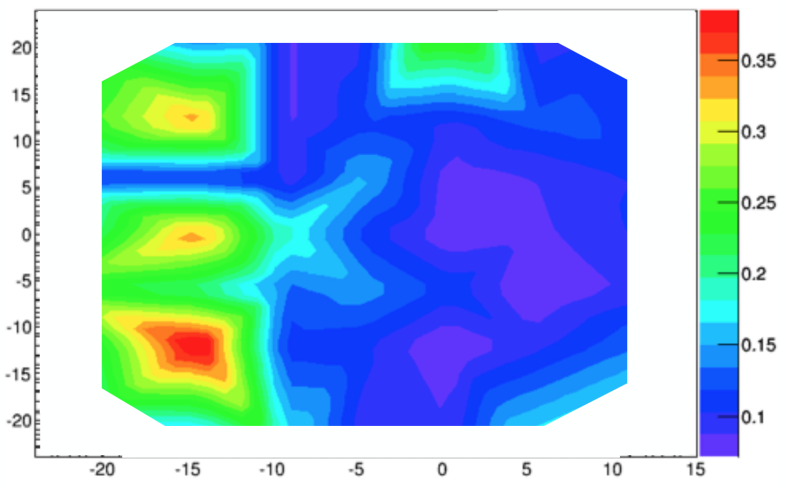

(b) $\sigma$

Figure 5.14: $\mu$ and $\sigma$ used to smear the simulated events interpolated from the overlapping 49 regions of the calorimeter kinematic setting 483.

This fitting procedure results in a $\chi^{2}$ for each of the 1,000,000 combinations of $\mu$ and $\sigma$. The minimal $\chi^{2}$ value corresponds to the $\mu$ and $\sigma$ that provide the best fit for the given region of the calorimeter. These values are saved and the procedure is repeated for all 49 regions.

After $\mu$ and $\sigma$ are found for all calorimeter regions, the simulation is smeared/calibrated for the whole calorimeter. This is accomplished event by event, using an interpolated $\mu$ and $\sigma$ according to the values found for the different regions. 


\section{Chapter 6}

\section{The DVCS cross section extraction}

Chapter 5 detailed the important analysis cuts and corrections that must be applied to the experimental data before extracting the DVCS cross section. This chapter discusses how the DVCS cross section is extracted from the data, and how the missing mass cut is chosen to minimize systematic uncertainty. All experimental cuts and corrections are assumed to have already been applied.

\subsection{Compton Form Factor Combination (CFFC) pa- rameterization of the DVCS Cross Section}

\subsubsection{Harmonic Expansion Coefficients}

Combining the equations from Sections 1.3.12, 1.3.10, 1.3.11, and 1.3.8, and subtracting the $\mathrm{BH}$ contribution, we have the following expressions of the unpolarized and polarized DVCS cross sections:

$$
\begin{aligned}
d^{5} \sigma_{\text {unpol }}= & \frac{1}{2}\left(\overrightarrow{d^{5} \sigma}+\overleftarrow{d^{5} \sigma}\right) \\
= & \frac{\alpha^{2} x_{B} y^{2}}{16 \pi^{2} Q^{2} \sqrt{1+\epsilon^{2}}}\left\{\left[c_{0}^{D V C S}+c_{1}^{D V C S} \cos \left(\phi_{\gamma \gamma}\right)+c_{2}^{D V C S} \cos \left(2 \phi_{\gamma \gamma}\right)\right]\right\}+ \\
& \frac{e^{6}}{y^{3} x_{B} t \mathcal{P}_{1}\left(\phi_{\gamma \gamma}\right) \mathcal{P}_{2}\left(\phi_{\gamma \gamma}\right)}\left\{\left[c_{0}^{I}+c_{1}^{I} \cos \left(\phi_{\gamma \gamma}\right)+c_{2}^{I} \cos \left(2 \phi_{\gamma \gamma}\right)+c_{3}^{I} \cos \left(3 \phi_{\gamma \gamma}\right)\right]\right\}( \\
\Delta^{5} \sigma_{\text {pol }}= & \frac{1}{2}\left(\overrightarrow{d^{5} \sigma}-\overleftarrow{d^{5} \sigma}\right) \\
= & \frac{\alpha^{2} x_{B} y^{2}}{16 \pi^{2} Q^{2} \sqrt{1+\epsilon^{2}}}\left\{\left[s_{1}^{D V C S} \sin \left(\phi_{\gamma \gamma}\right)+s_{2}^{D V C S} \sin \left(2 \phi_{\gamma \gamma}\right)\right]\right\}+ \\
& \frac{e^{6}}{y^{3} x_{B} t \mathcal{P}_{1}\left(\phi_{\gamma \gamma}\right) \mathcal{P}_{2}\left(\phi_{\gamma \gamma}\right)}\left\{\left[s_{1}^{I} \sin \left(\phi_{\gamma \gamma}\right)+s_{2}^{I} \sin \left(2 \phi_{\gamma \gamma}\right)+s_{3}^{I} \sin \left(3 \phi_{\gamma \gamma}\right)\right]\right\}
\end{aligned}
$$




\begin{tabular}{|l|c|c|c|c|}
\hline Combination & Twist & $\phi$ Dependence & Pol/Unpol & Used in Fit \\
\hline $\mathcal{C}^{D V C S}\left(\mathcal{F}_{++}, \mathcal{F}_{++}^{*} \mid \mathcal{F}_{+-}, \mathcal{F}_{+-}^{*}\right)$ & twist-2 & constant & unpol & $\checkmark$ \\
\hline $\mathcal{C}^{D V C S}\left(\mathcal{F}_{0+}, \mathcal{F}_{0+}^{*}\right)$ & twist-3 & constant & unpol & \\
\hline $\operatorname{Re} \mathcal{C}^{I, V}\left(\mathcal{F}_{++}\right)$ & "twist-3" & constant & unpol & \\
\hline $\operatorname{Re} \mathcal{C}^{I}\left(\mathcal{F}_{++}\right)$ & twist-2 & $\cos \phi_{\gamma \gamma}$ & unpol & $\checkmark$ \\
\hline $\operatorname{Re} \mathcal{C}^{D V C S}\left(\mathcal{F}_{0+} \mid \mathcal{F}_{++}^{*}, \mathcal{F}_{+-}^{*}\right)$ & twist-3 & $\cos \phi_{\gamma \gamma}$ & unpol & \\
\hline $\operatorname{Re} \mathcal{C}^{I, A}\left(\mathcal{F}_{++}\right)$ & "twist-3" & $\cos \phi_{\gamma \gamma}$ & unpol & \\
\hline $\operatorname{Re} \mathcal{C}^{I}\left(\mathcal{F}_{0+}\right)$ & twist-3 & $\cos 2 \phi_{\gamma \gamma}$ & unpol & $\checkmark$ \\
\hline $\operatorname{Re} \mathcal{C}^{D V C S}\left(\mathcal{F}_{+-}, \mathcal{F}_{+-}^{*}\right)$ & twist-3 & $\cos 2 \phi_{\gamma \gamma}$ & unpol & \\
\hline $\operatorname{Im} \mathcal{C}^{I}\left(\mathcal{F}_{++}\right)$ & twist-2 & $\sin \phi_{\gamma \gamma}$ & pol & $\checkmark$ \\
\hline $\operatorname{Im} \mathcal{C}^{D V C S}\left(\mathcal{F}_{0+} \mid \mathcal{F}_{++}^{*}, \mathcal{F}_{+-}^{*}\right)$ & twist-3 & $\sin \phi_{\gamma \gamma}$ & pol & \\
\hline $\operatorname{Im} \mathcal{C}^{I, V}\left(\mathcal{F}_{++}\right)$ & "twist-3" & $\sin \phi_{\gamma \gamma}$ & pol & \\
\hline $\operatorname{Im} \mathcal{C}^{I, A}\left(\mathcal{F}_{++}\right)$ & "twist-3" & $\sin \phi_{\gamma \gamma}$ & pol & \\
\hline $\operatorname{Im} \mathcal{C}^{I}\left(\mathcal{F}_{0+}\right)$ & twist-3 & $\sin 2 \phi_{\gamma \gamma}$ & pol & $\checkmark$ \\
\hline
\end{tabular}

Table 6.1: A table of the CFFCs that can be used to parametrize the DVCS cross section. The notation $\mathcal{F}_{a b}$ follows from the helicity CFFs defined in section 1.3.1, $\mathcal{C}$ means combination, and the superscript DVCS and I refer to CFFCs that contribute to the pure DVCS term and the BH-DVCS interference term, respectively. The Pol/Unpol column denotes if the CFFC is accessible from the unpolarized or polarized cross section. The superscripts $A$ and $V$ relate to the vector and axial vector functions from equation 1.17 . More details on the $A$ and $V$ terms can be found in [17].

The BH-DVCS interference term has a more complex $\phi$ dependence due to the factors $\mathcal{P}_{1}\left(\phi_{\gamma \gamma}\right)$ and $\mathcal{P}_{2}\left(\phi_{\gamma \gamma}\right)$ which arise from the electron propagator. These terms are given in Appendix A. For fixed bins in $t$, the $\phi_{\gamma \gamma}$ dependence of the data can be used to extract the harmonic coefficients $c_{n}^{D V C S}, s_{n}^{D V C S}, c_{n}^{I}$, and $s_{n}^{I}$.

\subsubsection{Choice of CFFCs}

The possible CFFCs that we can use to parameterize the DVCS cross section are given in table 6.1. The choice was made to use the CFFCs as noted in the table.

The quoted "twist-3" in the table means that the combination is twist-2, but kinematically suppressed like a twist-3. The first subscripted,+ 0 , or - represents the helicity state of the incoming virtual photon, and the second + or - represents the helicity of the outgoing real photon. Note that only one CFFC is chosen for each $\phi_{\gamma \gamma}$ dependence (color-coded) from the table 6.1. This is done so we can extract a single CFFC with that $\phi_{\gamma \gamma}$ dependence. Although we are really extracting the sum of all terms with that $\phi_{\gamma \gamma}$ dependence from the data, we treat it as the single term. To extract the combinations individually from the sum, we would need experimental data at the same $\xi$ and $t$, but $n$ different beam energies or $Q^{2}$. Future DVCS experiments are planned to take this data. Note that the $\phi_{\gamma \gamma}$ dependence introduced from the propagators $\mathcal{P}_{1}$ and $\mathcal{P}_{2}$ in the interference term is not enough to be able 
to separate the terms [66] [67].

Although all of the CFFCs in table 6.1 contribute to the cross section, the $\phi_{\gamma \gamma}$ dependence can be accurately captured using only a single combination with each $\phi_{\gamma \gamma}$ dependence. Because we only fit one term with each $\phi_{\gamma \gamma}$ dependence, the extracted values for the CFFCs (section 6.4) are not exact, and would have different values if all the terms were included in the fit. However, the choice was made to keep the most dominant term with each $\phi_{\gamma \gamma}$ dependence, and twist-3 contributions are expected to be small, so the real values of the chosen CFFCs are expected to be close to those extracted with this analysis. A goal of future DVCS experiments will be to extract all CFFC terms from the measured cross section. Appendix A contains the definitions for these CFFC terms.

With this choice of CFFCs, the $s_{n}^{D V C S}$ terms in Equation 6.2, and the $c_{1,2}^{D V C S}$ terms in Equation 6.1 do not contribute to the cross section and are thus taken to be zero. The unpolarized and polarized cross sections are then expressed as:

$$
\begin{gathered}
d^{5} \sigma_{\text {unpol }}=\frac{\alpha^{2} x_{B} y^{2}}{16 \pi^{2} Q^{2} \sqrt{1+\epsilon^{2}}} c_{0}^{D V C S}+\frac{e^{6}}{y^{3} x_{B} t \mathcal{P}_{1}\left(\phi_{\gamma \gamma}\right) \mathcal{P}_{2}\left(\phi_{\gamma \gamma}\right)}\left\{\left[c_{0}^{I}+c_{1}^{I} \cos \left(\phi_{\gamma \gamma}\right)+c_{2}^{I} \cos \left(2 \phi_{\gamma \gamma}\right)+c_{3}^{I} \cos \left(3 \phi_{\gamma \gamma}\right)\right]\right\} \\
\Delta^{5} \sigma_{\text {pol }}=\frac{e^{6}}{y^{3} x_{B} t \mathcal{P}_{1}\left(\phi_{\gamma \gamma}\right) \mathcal{P}_{2}\left(\phi_{\gamma \gamma}\right)}\left\{\left[s_{1}^{I} \sin \left(\phi_{\gamma \gamma}\right)+s_{2}^{I} \sin \left(2 \phi_{\gamma \gamma}\right)+s_{3}^{I} \sin \left(3 \phi_{\gamma \gamma}\right)\right]\right\}
\end{gathered}
$$

For this choice of CFFCs, the coefficients in Equations 6.3 and 6.4 are given as [17]:

$$
\begin{aligned}
& c_{0}^{D V C S}=C_{++, *++\mid+-, *+-}(0) \mathcal{C}^{D V C S}\left(\mathcal{F}_{++} \mathcal{F}_{++}^{*} \mid \mathcal{F}_{+-} \mathcal{F}_{+-}^{*}\right) \\
& c_{0}^{I}=C_{++}(0) \operatorname{ReC}^{I}\left(F_{++}\right)+C_{0+}(0) \operatorname{ReC}^{I}\left(F_{0+}\right) \\
& c_{1}^{I}=C_{++}(1) \operatorname{Re}^{I}\left(F_{++}\right)+C_{0+}(1) \operatorname{ReC}^{I}\left(F_{0+}\right) \\
& c_{2}^{I}=C_{++}(2) \operatorname{ReC}^{I}\left(F_{++}\right)+C_{0+}(2) \operatorname{ReC}^{I}\left(F_{0+}\right) \\
& c_{3}^{I}=C_{++}(3) \operatorname{ReC}^{I}\left(F_{++}\right)
\end{aligned}
$$

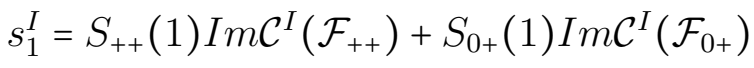




$$
s_{2}^{I}=S_{++}(2) \operatorname{Im} \mathcal{C}^{I}\left(\mathcal{F}_{++}\right)+S_{0+}(2) \operatorname{Im} \mathcal{C}^{I}\left(\mathcal{F}_{0+}\right)
$$

and according to [17]:

$$
s_{3}^{I}=0
$$

Here the functions $C_{++, *++\mid+, *+-}(0), C_{++}(n), C_{0+}(n), S_{++}(n)$, and $S_{0+}(n)$ depend on kinematic variables $E, x_{B}, Q^{2}, t, \phi_{\gamma \gamma}, \lambda(\mathrm{S}(\mathrm{n})$ only) and are given explicitly in Appendix A. It is important to note that with the expressions for the $c_{n}$ and $s_{n}$ above, the expressions for the cross sections given in Equations 6.3 and 6.4 can be written in the general form:

$$
\begin{gathered}
d^{5} \sigma_{\text {unpol }}=F_{1} \mathcal{C}^{D V C S}\left(\mathcal{F}_{++} \mathcal{F}_{++}^{*} \mid \mathcal{F}_{+-} \mathcal{F}_{+-}^{*}\right)+F_{2} \operatorname{Re} \mathcal{C}\left(\mathcal{F}_{++}\right)+F_{3} \operatorname{Re} \mathcal{C}\left(\mathcal{F}_{0+}\right) \\
\Delta^{5} \sigma_{\text {pol }}=F_{4} \operatorname{Im} \mathcal{C}\left(\mathcal{F}_{++}\right)+F_{5} \operatorname{Im} \mathcal{C}\left(\mathcal{F}_{0+}\right)
\end{gathered}
$$

where the terms $F_{n}$ encompass all kinematic prefactors that appear in front of the corresponding $\mathrm{CFFC}$. For this choice of CFFCs, the terms $F_{n}$ are given by the following:

$$
\begin{gathered}
F_{1}=\frac{\alpha^{2} x_{B} y^{2}}{8 \pi Q^{2} \sqrt{1+\epsilon^{2}}}\left[C_{++, *++\mid+, *+-}(0)\right] \\
F_{2}=\frac{e^{6}}{y^{3} x_{B} t \mathcal{P}_{1}\left(\phi_{\gamma \gamma}\right) \mathcal{P}_{2}\left(\phi_{\gamma \gamma}\right)}\left[C_{++}(0)+C_{++}(1) \cos \phi_{\gamma \gamma}+C_{++}(2) \cos 2 \phi_{\gamma \gamma}+C_{++}(3) \cos 3 \phi_{\gamma \gamma}\right] \\
F_{3}=\frac{e^{6}}{y^{3} x_{B} t \mathcal{P}_{1}\left(\phi_{\gamma \gamma}\right) \mathcal{P}_{2}\left(\phi_{\gamma \gamma}\right)}\left[C_{0+}(0)+C_{0+}(1) \cos \phi_{\gamma \gamma}+C_{0+}(2) \cos 2 \phi_{\gamma \gamma}\right] \\
F_{4}=\frac{e^{6}}{y^{3} x_{B} t \mathcal{P}_{1}\left(\phi_{\gamma \gamma}\right) \mathcal{P}_{2}\left(\phi_{\gamma \gamma}\right)}\left[S_{++}(1) \sin \phi_{\gamma \gamma}+S_{++}(2) \sin 2 \phi_{\gamma \gamma}\right] \\
F_{5}=\frac{e^{6}}{y^{3} x_{B} t \mathcal{P}_{1}\left(\phi_{\gamma \gamma}\right) \mathcal{P}_{2}\left(\phi_{\gamma \gamma}\right)}\left[S_{0+}(1) \sin \phi_{\gamma \gamma}+S_{0+}(2) \sin 2 \phi_{\gamma \gamma}\right]
\end{gathered}
$$

Finally, if we take:

$$
X_{1}\left(t, \xi, Q^{2}\right)=\mathcal{C}^{D V C S}\left(\mathcal{F}_{++} \mathcal{F}_{++}^{*} \mid \mathcal{F}_{+-} \mathcal{F}_{+-}^{*}\right)
$$




$$
\begin{aligned}
& X_{2}\left(t, \xi, Q^{2}\right)=\operatorname{Re}^{I}\left(\mathcal{F}_{++}\right) \\
& X_{3}\left(t, \xi, Q^{2}\right)=\operatorname{Re} \mathcal{C}^{I}\left(\mathcal{F}_{0+}\right) \\
& X_{4}\left(t, \xi, Q^{2}\right)=\operatorname{Im} \mathcal{C}^{I}\left(\mathcal{F}_{++}\right) \\
& X_{5}\left(t, \xi, Q^{2}\right)=\operatorname{Im} \mathcal{C}^{I}\left(\mathcal{F}_{0+}\right)
\end{aligned}
$$

we can write Equations 6.13 and 6.14 as:

$$
\begin{gathered}
d^{5} \sigma_{\text {unpol }}=\sum_{n=1}^{3} F_{n}\left(E, x_{B}, Q^{2}, t, \phi_{\gamma \gamma}\right) X_{n}\left(t, \xi, Q^{2}\right) \\
\Delta^{5} \sigma_{\text {pol }}=\sum_{n=4}^{5} F_{n}\left(E, x_{B}, Q^{2}, t, \phi_{\gamma \gamma}, \lambda\right) X_{n}\left(t, \xi, Q^{2}\right)
\end{gathered}
$$

We use a fitting procedure to extract $\mathcal{N}=5$ CFFCs, $X_{n}$, from experimental data.

\subsection{Principle of the Extraction}

A fitting procedure is used to extract CFFCs and their contributions to the DVCS cross section. These contributions are then used to reconstruct the measured experimental cross section. This procedure will be detailed in the following sections.

\subsubsection{Binning Formalism}

The measured cross section is extracted in 120 bins for each kinematic setting. The experimental events passing all analysis cuts are binned according to their $t-t_{\min }$ value ( 5 bins), and their $\phi_{\gamma \gamma}$ value (24) in each $t-t_{m i n}$-bin.

Because these are experimental data, these values of $t-t_{\min }$ and $\phi_{\gamma \gamma}$ are not the actual values the event had at the scattering vertex, but rather the reconstructed values from the detectors. These 120 ( $5 \times 24)$ reconstructed bins $\mathcal{R}$ are referred to as the reconstructed bins in this section. In this section, we will refer to the "vertex kinematics" as the true kinematics quantities, as opposed to the "reconstructed kinematics".

The vertex kinematics are those relevant to the cross section. To quantify this difference, we use a Monte Carlo simulation to access the kinematics of events at the scattering vertex, and their corresponding reconstructed kinematics. Using the Monte Carlo allows for the accounting of bin migration from vertex to reconstructed $t$ and $\phi_{\gamma \gamma}$ bins when extracting the DVCS cross sections. We choose 5 vertex bins $\mathcal{V}$ in $t-t_{\min }$. 


\subsubsection{Experimental Number of Events}

For a given reconstructed bin $r$, the experimental number of events $N_{r}^{\exp }$ in that bin, is related to that bin's average cross section $\left\langle\frac{d^{5} \sigma}{d x_{B} d Q^{2} d t d \phi_{\gamma \gamma} d \phi_{e}}\right\rangle_{r}$ by:

$$
N_{r}^{e x p}=\mathcal{L} \int_{x_{B}, Q^{2}, t, \phi_{\gamma \gamma}, \phi_{e}} \frac{d^{5} \sigma}{d x_{B} d Q^{2} d t d \phi_{\gamma \gamma} d \phi_{e}} d x_{B} d Q^{2} d t d \phi_{\gamma \gamma} d \phi_{e}=\mathcal{L}\left\langle\frac{d^{5} \sigma}{d x_{B} d Q^{2} d t d \phi_{\gamma \gamma} d \phi_{e}}\right\rangle_{r} \Gamma_{r}
$$

where $\mathcal{L}$ is the integrated luminosity and $\Gamma_{r}$ an experimental acceptance term for bin $r$. This equation is reminiscent of the equivalent DIS expression in Equation 4.1. Here, $\Gamma_{r}$ is found from the simulation for each event according to:

$$
\Gamma_{r}=\frac{1}{N_{M C}^{g e n}} \sum_{i=1}^{N_{M C, r}}\left(\Delta x_{B} \Delta Q^{2} \Delta t \Delta \phi_{\gamma \gamma} \Delta \phi_{e}\right)_{i}
$$

where $N_{M C}^{g e n}$ is the number of generated events in the simulation, $N_{M C, r}$ is the number of simulated events passing all cuts in bin $r$, and $\left(\Delta x_{B} \Delta Q^{2} \Delta t \Delta \phi_{\gamma \gamma} \Delta \phi_{e}\right)_{i}$ is the phase space factor for event $i$. The $\Delta$ notation signifies the range of values for each variable used in the Monte Carlo. Using equations 6.27 and 6.28 we can find the average cross section for each bin:

$$
\left\langle\frac{d^{5} \sigma}{d x_{B} d Q^{2} d t d \phi_{\gamma \gamma} d \phi_{e}}\right\rangle_{r}=\frac{N_{r}^{\exp }}{\mathcal{L} * \Gamma_{r}}
$$

We consider two sets of $N_{r}$ : $N_{r}=N_{r,+}+N_{r,-}$ used to extract the helicity-independent cross section, and $N_{r}=N_{r,+}-N_{r,-}$ used to extract the helicity-dependent cross section, where $N_{r,+}$ is the experimental number of events in bin $\mathrm{r}$ with a spin aligned electron beam, and $N_{r,-}$ the events with spin anti-aligned electron beam.

\subsubsection{Fitting the CFFCs}

The DVCS cross section $\frac{d^{5} \sigma}{d x_{B} d Q^{2} d t d \phi_{\gamma \gamma} d \phi_{e}}$ is parameterized by CFFCs. We can generalize the results shown in equations 6.25 and 6.26 for a choice of $\mathcal{N}=5$ CFFCs:

$$
\frac{d^{5} \sigma}{d x_{B} d Q^{2} d t d \phi_{\gamma \gamma} d \phi_{e}}=\sum_{n=1}^{\mathcal{N}} F_{n}\left(E, x_{B}, Q^{2}, t, \phi_{\gamma \gamma}, \lambda\right) X_{n}\left(t, \xi, Q^{2}\right)
$$

We assume the CFF combination $X_{n}$ is constant within each bin $r$, and we arrive at the following relationship between the number of DVCS events $N_{r}^{D V C S}$ and $\left\langle X_{n, r}\right\rangle$ :

$$
N_{r}^{D V C S}=\mathcal{L} \sum_{n=1}^{\mathcal{N}}\left\langle X_{n, r}\right\rangle \int_{\Phi} F_{n}\left(E, x_{B}, Q^{2}, t, \phi_{\gamma \gamma}, \lambda\right) d \Phi
$$




\subsubsection{Fitting Procedure}

To extract the CFFCs, we fit the number of simulated DVCS events $N_{r}^{M C}(\mathrm{Eq} 6.31)$ in each bin $r$ to the experimental number of events $N_{r}^{e x p}(\mathrm{Eq} 6.27)$ in the same reconstructed bin. However, we want to extract CFFCs at the vertex, so we need to modify equation 6.31. We define a matrix $\mathcal{K}$ whose elements $\mathcal{K}_{r v}$ represent the probability of an event in vertex bin $v$ to migrate to reconstructed bin $r$ :

$$
N_{r}^{M C}=\sum_{v=1}^{\mathcal{V}} \mathcal{K}_{r v} N_{v}^{M C}
$$

We modify equation 6.31 to account for the bin migration effects:

$$
N_{r}^{M C}=\mathcal{L} \sum_{v=1}^{\mathcal{V}} \sum_{n=1}^{\mathcal{N}}\left\langle X_{n, v}\right\rangle \int_{\Phi_{v}} \mathcal{K}_{r v} F_{n}\left(E, x_{B, v}, Q_{v}^{2}, t_{v}, \phi_{\gamma \gamma, v}\right) d \Phi_{v}
$$

Note that the $F_{n}$ are now evaluated using vertex kinematics, and the $X_{n}$ are extracted as a function of vertex kinematics.

\subsubsection{Bethe-Heitler Subtraction}

The unpolarized extracted cross section from Section 6.2.2 contains the Bethe-Heitler(BH) term, linear CFFCs from the DVCS-BH interference term (real coefficients), and bilinear CFFCs from the DVCS amplitude term, as described in Section 1.3.10, The polarized extracted cross section contains only the DVCS-BH interference term (imaginary coefficients).

Because the unpolarized cross section contains the BH term, we must subtract this before performing the fit to extract CFFCs. The BH contribution is calculated for every simulated event that passes all analysis cuts. Note that the BH for each event is calculated using vertex, and not the reconstructed, kinematics. After binning our simulated events into the same reconstructed bins as the experimental data, we subtract the total $\mathrm{BH}$ contribution from the experimental number of events in each bin:

$$
N_{r, B H s u b}^{e x p}=N_{r}-\sum_{i=1}^{N_{r}^{M C}} \frac{\mathcal{L} * B H\left(E, Q_{v}^{2}, x_{B, v}, t_{v}, \phi_{\gamma \gamma v}\right)}{\Gamma_{r}}
$$

It is assumed throughout this documentation of the fitting procedure that $N_{r}^{e x p}$ now represents the $\mathrm{BH}$-subtracted number of events for helicity-independent fit.

\subsubsection{Calculation of the matrix $\mathcal{K}$}

The matrix elements $\mathcal{K}_{r v}$ from equation 6.33 must be calculated before performing the fit. We can extend the definition of $\mathcal{K}$ to include the CFFCs:

$$
\mathcal{K}_{r v}^{n}=\int_{\Phi_{v}} K_{r v} F_{n}\left(E, x_{B, v}, Q_{v}^{2}, t_{v}, \phi_{\gamma \gamma v}\right) d \Phi_{v}
$$


where the elements $\mathcal{K}_{r v}^{n}$ are calculated from the Monte Carlo events that fall in the vertex kinematics bin $v$ and reconstructed kinematics bin $r$. Using our simulated data, we can calculate the elements of $\mathcal{K}_{r v}^{n}$ using:

$$
\mathcal{K}_{r v}^{n}=\sum_{i \in(v \cap r)} F_{n}\left(E,\left(x_{B, v}\right)_{i},\left(Q_{v}^{2}\right)_{i},\left(t_{v}\right)_{i}, \phi_{\gamma \gamma i}\right) \Gamma_{i}
$$

This allows us to rewrite equation 6.33 as:

$$
N_{r}^{M C}=\mathcal{L} \sum_{v=1}^{\mathcal{V}} \sum_{n=1}^{\mathcal{N}}\left\langle X_{n, v}\right\rangle \mathcal{K}_{r v}^{n}
$$

This relation can be unfolded to extract the CFFCs $X_{n}$ from the data binned using reconstructed kinematic quantities.

\subsubsection{CFFC Extraction}

To extract CFFCs, we find their values $\bar{X}_{n, v}$ that best fit $N_{r}^{M C}$ to $N_{r}^{\text {exp }}$ for all bins $\mathcal{R}$ simultaneously. To do this, we minimize the $\chi^{2}$ :

$$
\chi^{2}\left(\left\{\left\langle X_{n, v}\right\rangle\right\}\right)=\sum_{r=1}^{\mathcal{R}}\left(\frac{N_{r}^{e x p}-N_{r}^{M C}}{\sigma_{r}}\right)^{2}
$$

Where $\sigma_{r}$ is the statistical uncertainty associated with $N_{r}$. We minimize the $\chi_{d o f}^{2}$ according to:

$$
0=-\left.\frac{1}{2} \frac{\partial \chi^{2}}{\partial X_{n, v}}\right|_{\bar{X}_{n, v}}
$$

This leads to solving a matrix equation $A X=B$, with $A$ a matrix with dimensions $(\mathcal{N} \times \mathcal{V}) \times(\mathcal{N} \times \mathcal{V})$ and $B$ a column vector with $\mathcal{N} \times \mathcal{V}$ rows. The matrix coefficients are:

$$
\begin{aligned}
A_{v, v^{\prime}}^{n, n^{\prime}} & =\mathcal{L}^{2} \sum_{r=1}^{\mathcal{R}} \frac{\mathcal{K}_{r v}^{n} \mathcal{K}_{r v^{\prime}}^{n^{\prime}}}{\left(\sigma_{r}\right)^{2}} \\
B_{v}^{n} & =\mathcal{L} \sum_{r=1}^{\mathcal{R}} \frac{\mathcal{K}_{r v}^{n} N_{r}^{e x p}}{\left(\sigma_{r}\right)^{2}}
\end{aligned}
$$

The values $\bar{X}_{n, v}$ that yield the best fit are then given by:

$$
\bar{X}_{n v}=\sum_{v^{\prime}=1}^{\mathcal{V}} \sum_{n^{\prime}=1}^{N}\left(A^{-1}\right)_{v, v^{\prime}}^{n, n^{\prime}} B_{v^{\prime}}^{n^{\prime}}
$$




\subsubsection{Cross Section Reconstruction}

After we have extracted $\bar{X}_{n, v}$, we can reconstruct a fitted cross section, with vertex kinematics, averaged over each bin according to:

$$
\frac{d^{5} \sigma_{v}^{f i t}}{d Q^{2} d x_{B} d t d \phi d \phi_{e}}=\sum_{n=1}^{\mathcal{N}} F_{n}\left(E,\left\langle Q^{2}\right\rangle,\left\langle x_{B}\right\rangle,\langle t\rangle, \phi, \lambda\right)\left\langle X_{n, v}\right\rangle
$$

where $\left\langle x_{B}\right\rangle,\left\langle Q^{2}\right\rangle$, and $\langle t\rangle$ are the vertex kinematics bin averages, and $\phi_{\gamma \gamma}$ is the reconstructed kinematics $\phi_{\gamma \gamma}$ bin average. The $F_{n} * X_{n, v}$ combinations represent the contribution to the fitted cross section from each CFF combination $n$. Note that the BH contribution is also considered at this step for the fitted unpolarized cross section.

The cross section of the experimentally measured data is then related to equation 6.43 according to:

$$
\frac{d^{5} \sigma_{v}^{e x p}}{d Q^{2} d x_{B} d t d \phi_{\gamma \gamma} d \phi_{e}}=\frac{N_{r}^{e x p}}{N_{r}^{M C}} \frac{d^{5} \sigma_{v}^{f i t}}{d Q^{2} d x_{B} d t d \phi_{\gamma \gamma} d \phi_{e}}
$$

Note that the results in this document have already been integrated over $\phi_{e}$ :

$$
\frac{d^{4} \sigma_{v}^{f i t}}{d Q^{2} d x_{B} d t d \phi_{\gamma \gamma}}=\int_{\phi_{e}} \frac{d^{5} \sigma_{v}^{f i t}}{d Q^{2} d x_{B} d t d \phi_{\gamma \gamma} d \phi_{e}} d \phi_{e}
$$

\subsubsection{Choice of CFFCs $\mathcal{N}$}

The fitted and experimentally measured cross sections depend on the choice of CFFC parameterization used in the fit. The CFFCs chosen for the fit whose results are documented in this thesis have been shown to provide good fit results. It is worth noting that other CFFCs may also yield good fit results, and in fact this choice contributes to the overall systematic uncertainty. While this is not explored in this document, the analysis performed by Frederic Georges [63] with the same data implies we will have a $1 \%$ uncertainty for CFFC choice.

For fitting the polarized cross section, we take $\mathcal{N}=2$ combinations, and $\mathcal{N}=3$ for the unpolarized (see section 6.1).

\subsubsection{Results}

Table 6.2 lists the $\chi^{2}$ results of fitting the simulated events to the experimental number of events in each bin. The normalized $\chi^{2} / d o f$ are reasonably close to 1 , with better fit results arising for the polarized case.

Figure 6.1 shows an example of the unpolarized fitted number of events compared to the experimental number of events in each bin. The remainder of these results for the Kin 48 settings, for both helicity-dependent and independent fits, can be found in Appendix B. In general, as $\left|t-t_{\text {min }}\right|$ becomes large, limited calorimeter acceptance leads to fewer events to fit near $\phi_{\gamma \gamma}=0^{\circ}, 360^{\circ}$. Consequently, large statistical uncertainty is expected for cross sections extracted in these bins. 


\begin{tabular}{|c|c|c|}
\hline Kin Setting & $\chi_{\text {unpol }}^{2} /$ dof & $\chi_{\text {pol }}^{2} /$ dof \\
\hline 481 & 2.03 & 0.78 \\
482 & 1.35 & 0.94 \\
483 & 1.68 & 0.95 \\
484 & 1.39 & 1.02 \\
\hline
\end{tabular}

Table 6.2: The normalized $\chi^{2}$ for the $x_{B}=0.48$ kinematic settings. The degrees of freedom $d o f=\mathcal{R}-\mathcal{N} \mathcal{V}$. For the unpolarized cross section fit, dof $=120-3 \times 5=105$. For the polarized cross section fit, $d o f=120-2 \times 5=110$.
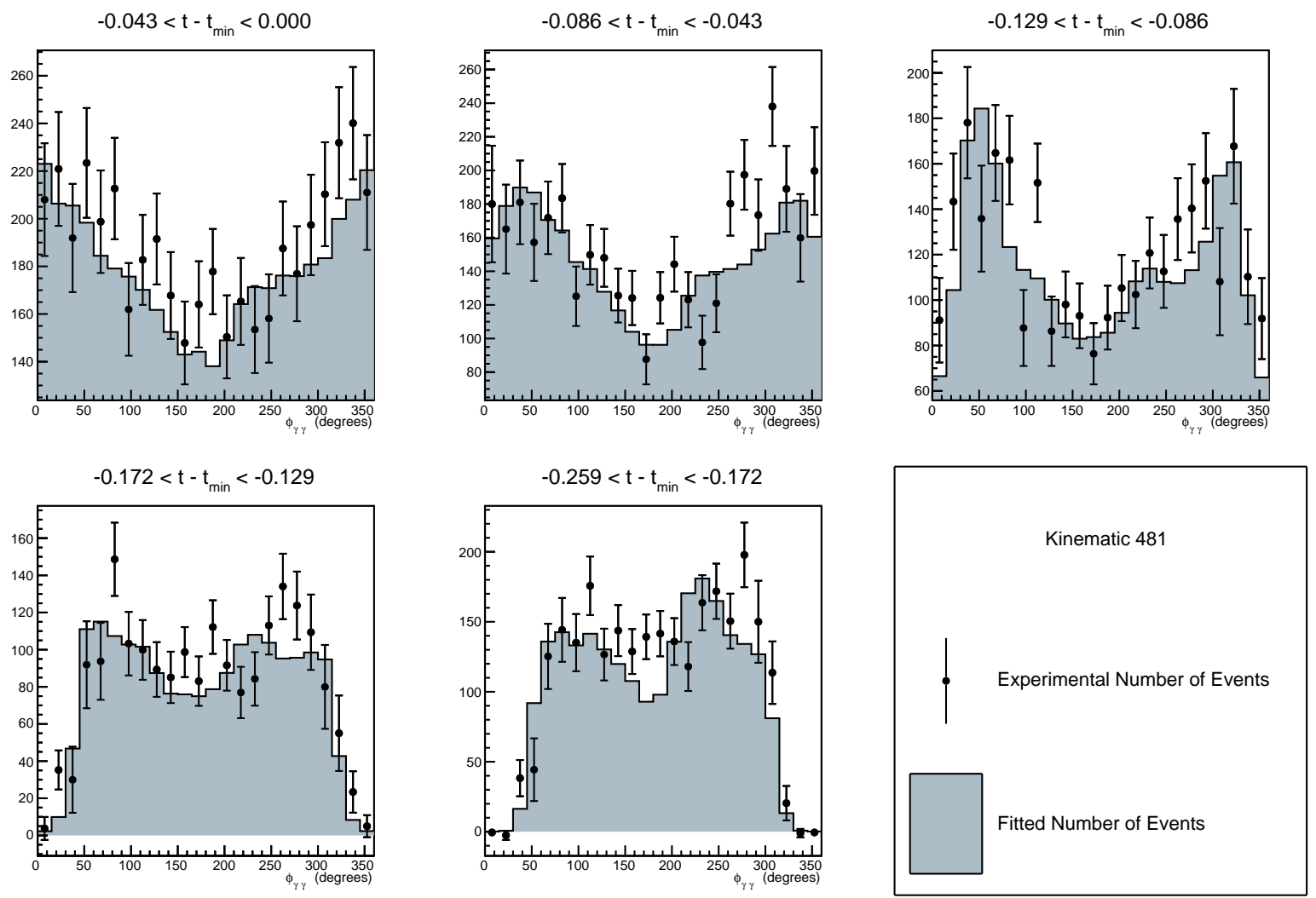

Figure 6.1: Unpolarized number of simulated events (grey filled histogram), plotted with the experimental number of events (black circles) after BH subtraction for kinematic setting 481. Error bars are statistical only.

Figure 6.2 shows an example of the extracted unpolarized cross sections in each bin. The remainder of these results for the Kin48 settings, for both helicity-dependent and independent extractions, can be found in Appendixrefap:CrossPlots. In these plots, the periwinkle line represents the Bethe-Heitler contribution to the cross section (unpolarized only), and the green, teal, and magenta bands represent the CFFC contributions (teal and purple for the helicity-dependent). Two models are shown for comparison in and blue. The grey 
band represents the fitted cross section, and the black dots represent the extracted cross section in each vertex kinematics bin.

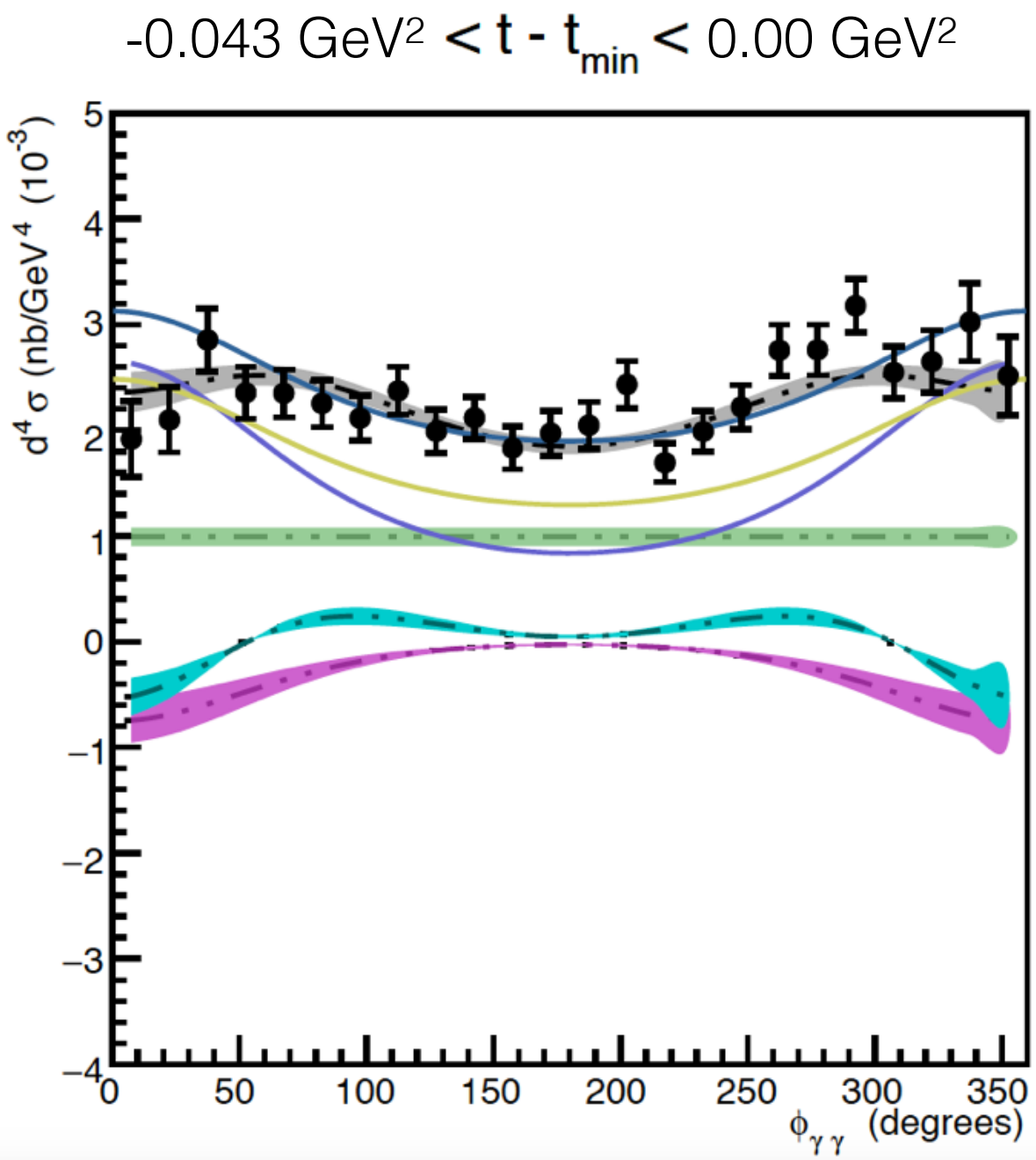

Figure 6.2: Unpolarized extracted cross section $\left(\times 10^{-3}\right)$ for a single $t$ bin of the kinematic setting 482, with the individual contributions from the $\mathrm{BH}$ and $\mathcal{N}=3 \mathrm{CFF}$ combinations $\left(X_{n, v} \times F_{n}\left(E,\left\langle Q^{2}\right\rangle,\left\langle x_{B}\right\rangle,\langle t\rangle, \phi_{\gamma \gamma}, \lambda\right)\right.$. The corresponding legend for this figure is in figure 6.3 below. 


\section{Kinematic 482}

\section{$\phi \quad$ Experimental Cross Section}

$-\cdots-\cdots$ Fitted Cross Section

\section{Bethe-Heitler

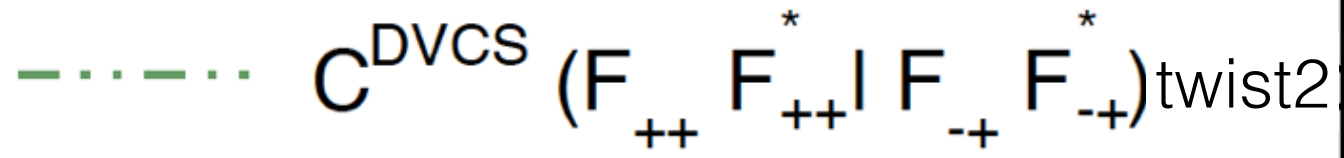 \\ -..-. $\operatorname{Re} C^{\prime}\left(\mathrm{F}_{++}\right)$twist2 \\ -..... Re $C^{\prime}\left(\mathrm{F}_{0_{+}}\right)$twist3 \\ KM10a Model}

\section{KM15 Model}

Figure 6.3: The legend corresponding to figure 6.2 .

The cross section is clearly distinguishable from the $\mathrm{BH}$ contribution, allowing us to draw conclusions about the CFFCs. At $\phi_{\gamma \gamma}$ close to $180^{\circ}$, the DVCS amplitude dominates, and as $\phi_{\gamma \gamma}$ approaches $0^{\circ}$ or $360^{\circ}$, the interference term becomes as dominant as the DVCS term. In general, the twist-3 contributions remain close to zero, while the twist-2 terms do not.

Figure 6.4 shows an example of the extracted polarized cross sections in each bin. The remainder of these results for the Kin48 settings, for both helicity-dependent and independent extractions, can be found in Appendixrefap:CrossPlots. This plot shows the twist-3 contribution as being small compared the to the twist-2. This also shows that our experimental cross section has good agreement with the KM15 model. 
$-0.124 \mathrm{GeV}^{2}<\mathrm{t}-\mathrm{t}_{\min }<-0.062 \mathrm{GeV}^{2}$

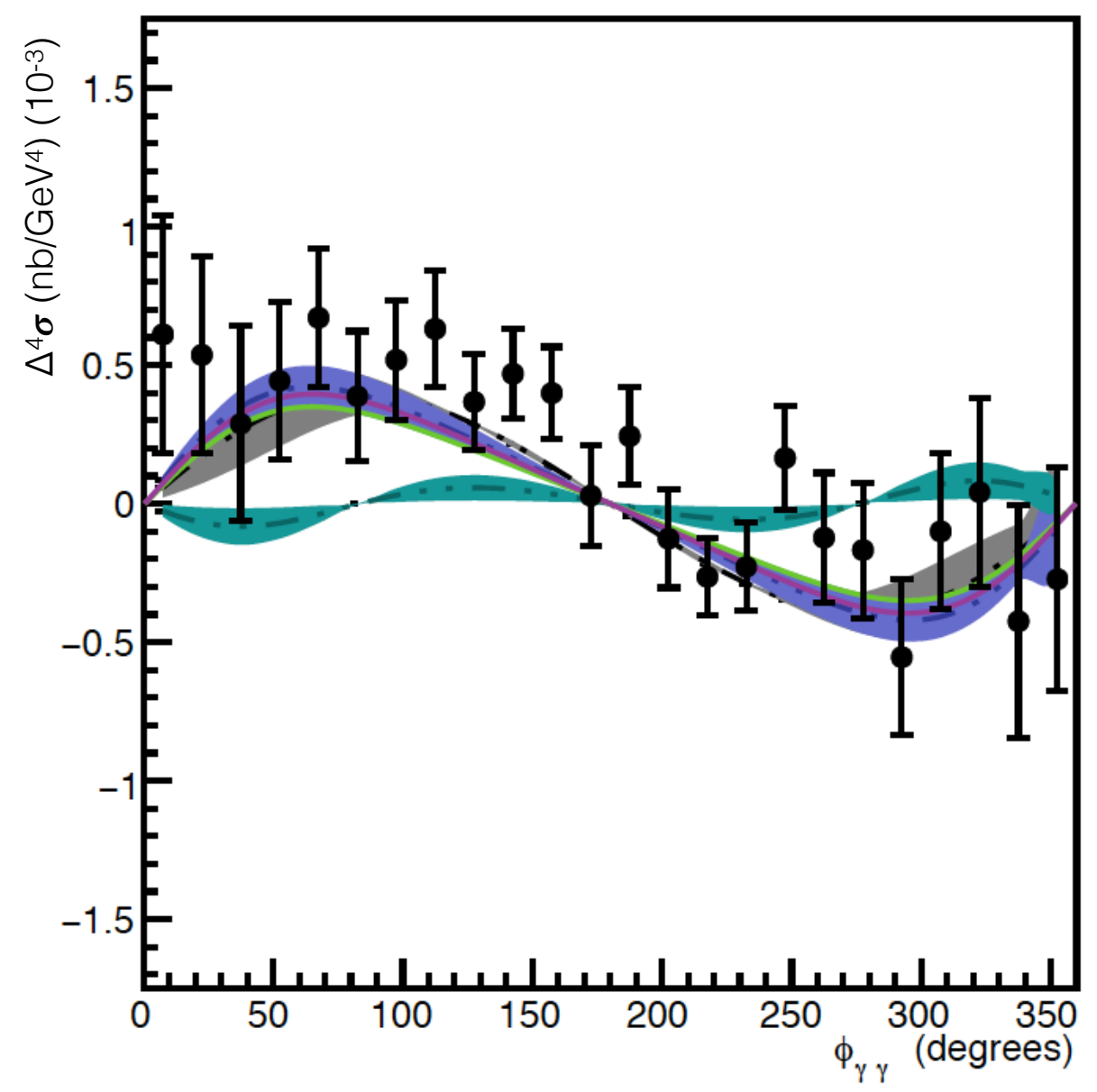

Figure 6.4: Polarized extracted cross section $\left(\times 10^{-3}\right)$ for a single $t$ bin of the kinematic setting 482, with the individual contributions from the $\mathrm{BH}$ and $\mathcal{N}=2 \mathrm{CFF}$ combinations $\left(X_{n, v} \times F_{n}\left(E,\left\langle Q^{2}\right\rangle,\left\langle x_{B}\right\rangle,\langle t\rangle, \phi_{\gamma \gamma}, \lambda\right)\right.$. The corresponding legend for this figure is in figure 6.5 below. 


\section{Kinematic 482}

$\uparrow \quad$ Experimental Cross Section

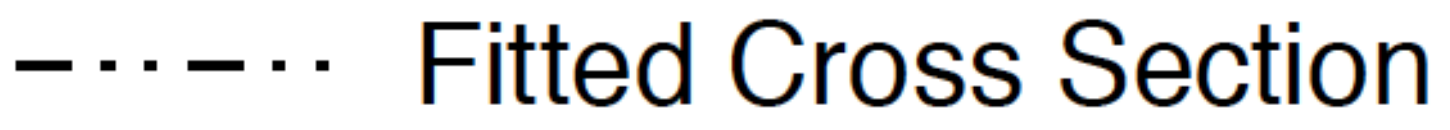

Im $C^{\prime}\left(F_{++}\right)$twist2

$\operatorname{Im} \mathrm{C}^{\prime}\left(\mathrm{F}_{0+}\right)$ twist3

KM10a Model

KM15 Model

Figure 6.5: The legend corresponding to figure 6.4 . 


\subsubsection{KM Models}

The comparison models referenced in the cross section results are the KM10a and KM15. These models are two global fits of the available DVCS data. The KM10a model does not include Hall A data. The KM15 models includes both Hall A and CLAS data up to and including the 2015 results. See references [75] and [76] for more details. Executables for obtaining the model data are found at http://calculon.phy.hr/gpd/.

In general, the unpolarized extracted cross sections show good agreement with the KM15 model. The kinematic setting 483 however shows good agreement with the KM10a model. For the extracted polarized cross sections, there is good agreement with both models.

\subsection{Missing Mass Cuts and Other Systematic Uncer- tainties}

The choice of missing mass cut introduces the largest uncertainty in the cross section extraction. As discussed previously in section 5.4.3.1, the SIDIS contamination in our data should begin at the theoretical $1.15 \mathrm{GeV}^{2}$ threshold in the missing mass distribution. However, because of photon energy and detector calibration uncertainties, this contamination begins below this limit. In addition to this, the simulation smearing procedure does not perfectly reproduce the gain and resolution of the physical calorimeter.

In figure 6.6 below, the missing mass distribution of smeared simulation is shown with the experimental data. Upper and lower missing mass cuts are chosen to include a region where the ratio of experimental to simulated events remains constant. 


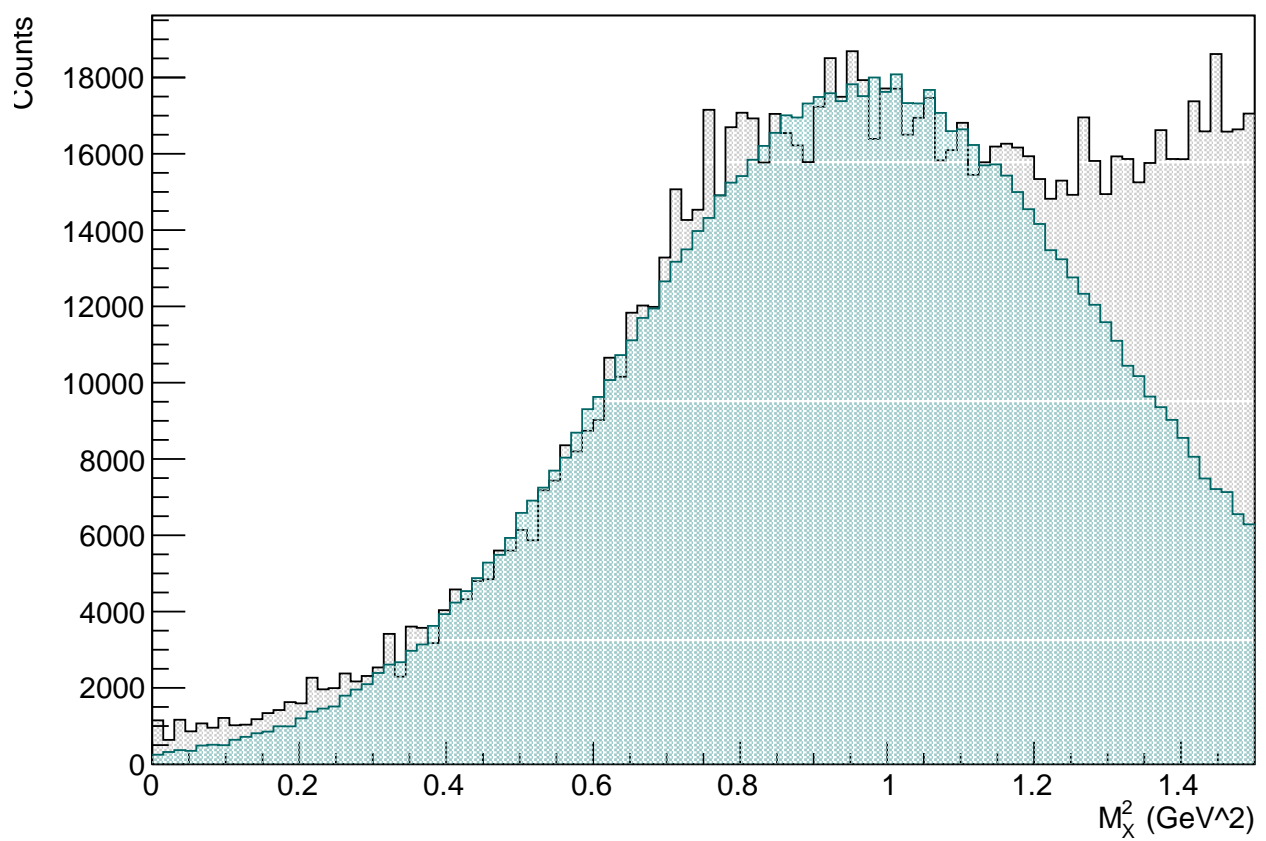

Figure 6.6: The smeared simulated events (teal) and the experimental number of events (grey) for kinematic setting 482. The chosen cuts for this kinematic setting are $0.45 \mathrm{GeV}^{2}$ and $1.05 \mathrm{GeV}^{2}$.

Table 6.3 below lists the upper and lower missing mass cuts determined for each kinematic setting.

\begin{tabular}{|c|c|c|}
\hline Kin Setting & Lower $M_{X}^{2}$ Cut $\left(\mathrm{GeV}^{2}\right)$ & Upper $M_{X}^{2}$ Cut $\left(\mathrm{GeV}^{2}\right)$ \\
\hline 481 & 0.45 & 1.05 \\
482 & 0.45 & 1.05 \\
483 & 0.3 & 1.00 \\
484 & 0.25 & 1.1 \\
\hline
\end{tabular}

Table 6.3: The selected missing mass cuts.

After the missing mass cuts are determined, the variation in the extracted cross section is measured in a given bin as the upper missing mass cut is varied from 0.8 to $1.5 \mathrm{GeV}^{2}$. Figure 6.7 shows an example of this procedure. The Monte Carlo is used to correct for DVCS events cut out by the cuts, but contamination of non-DVCS events affect the extracted cross section as the upper cut goes above $1.1 \mathrm{GeV}$. This plot provides verification that the cross section is stable around our chosen upper missing mass cut. The missing mass uncertainty introduced to the cross section is taken from its $\max / \min$ fluctuation around $\pm 0.06 \mathrm{GeV}^{2}$ of the chosen cut from table 6.3. 


\section{Missing Mass Uncertainty 482}

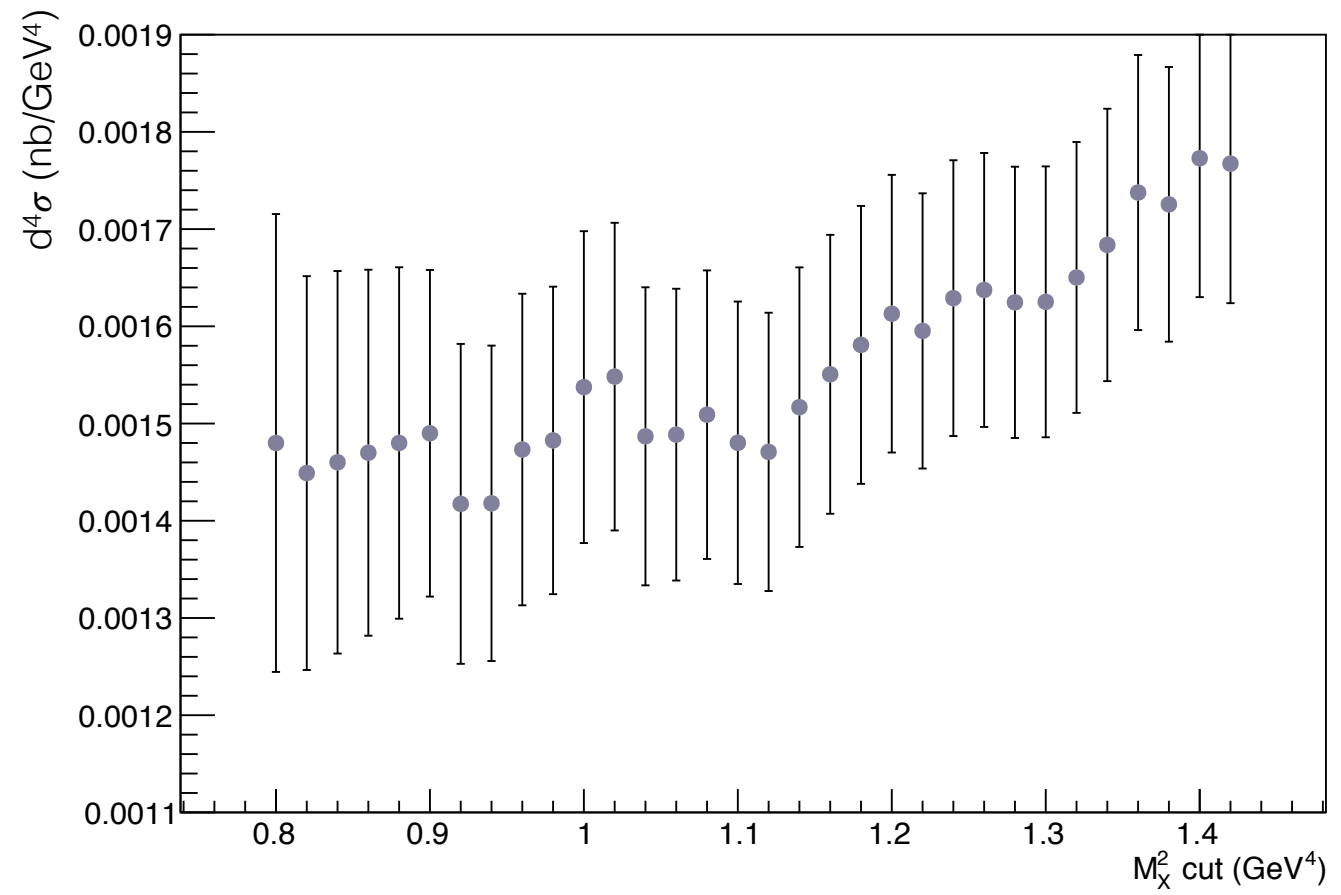

Figure 6.7: The change in unpolarized extracted cross section for a single bin as the upper missing mass cut is varied.

Figure 6.8 shows the results of performing the uncertainty calculation in each bin (in units of $\%$ difference from the nominal cross section extracted in that bin). The missing mass uncertainties for each bin are quoted in the tables of results (Appendix $\mathrm{D}$ for unpolarized and Appendix $\mathrm{E}$ for polarized). In general, for bins with good statistics, the measured uncertainty due to missing mass cuts is $2-6 \%$. 

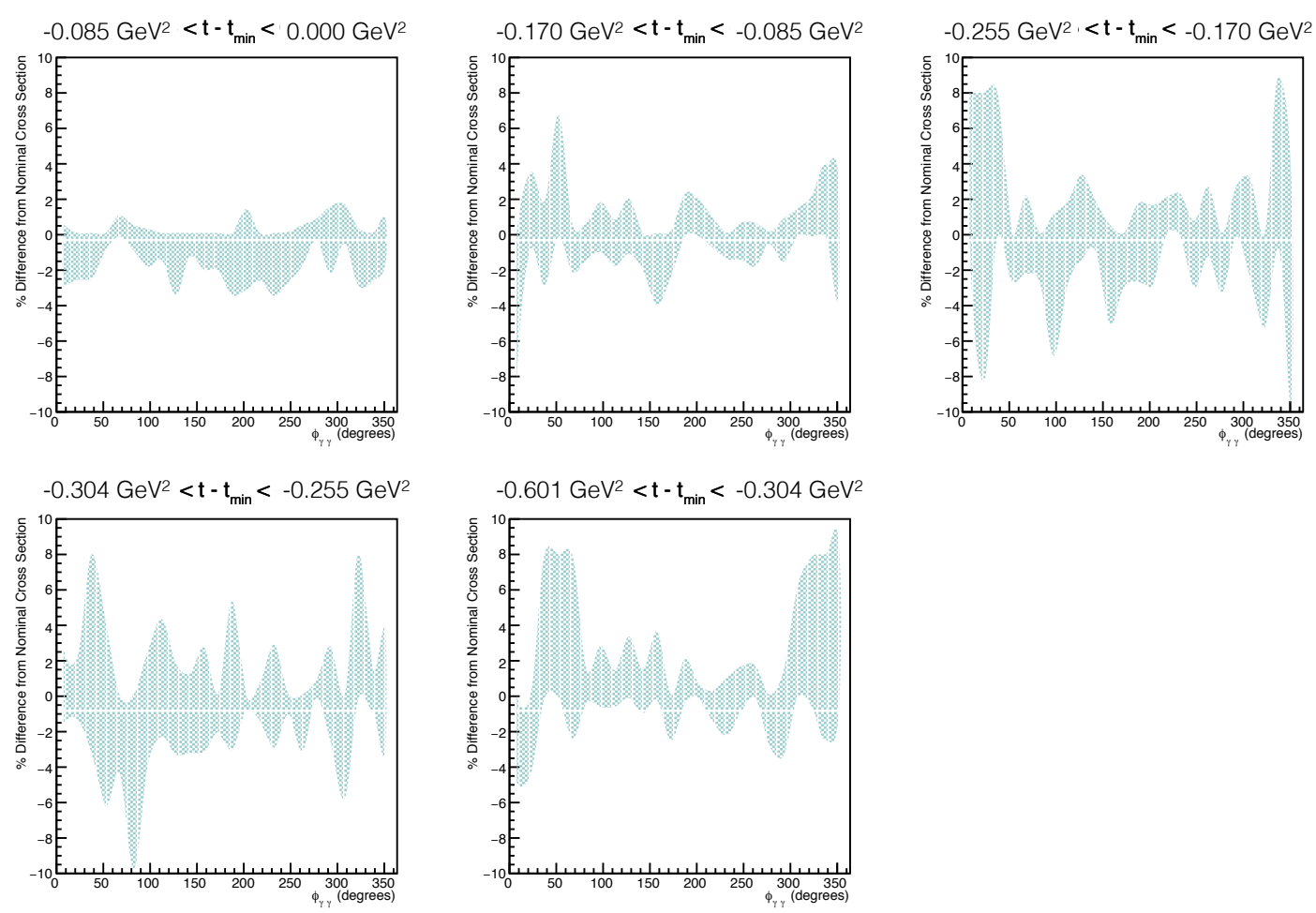

Figure 6.8: The \% change in unpolarized extracted cross section for all bins as the upper missing mass cut is varied by $\pm 0.06 \mathrm{GeV}^{2}$ for kinematic setting 484 .

\subsubsection{Systematic Uncertainties}

The systematic uncertainties incurred from analysis cuts, beam polarization, and radiative corrections are summarized in the table below.

\begin{tabular}{|c|c|}
\hline Systematic Uncertainty & Value (\%) \\
\hline HRS acceptance (R-error) & $0.67-2.23$ \\
HRS electron Identification & 0.5 \\
HRS multi-track correction & 0.5 \\
Luminosity and dead time & 1.6 \\
Virtual radiative corrections & 2.0 \\
CFF parametrization choice & 1.0 \\
Beam polarization & 1.00 \\
\hline \hline Total Unpolarized & $2.9-3.6$ \\
Total Polarized & $3.1-3.7$ \\
\hline
\end{tabular}

Table 6.4: Summary of systematic uncertainties. 


\subsubsection{Impact of DIS Results on DVCS}

As discussed in section 4.2, the DIS cross section extraction for the Kin-48 settings was as bad as 6-9\% below the expected values. Unfortunately, the reason this is the case is not well understood. As a consequence, it is not understood if the same problem would be present in DVCS data, and if so, how it could be corrected. It is possible that because of some analysis cuts, or HRS inefficiency, 6-9\% of the events were missed by data acquisition, and the DVCS number of events should be scaled to reflect this. However, because we do not understand the cause of the discrepancy in the DIS results, the kinematic distribution over which the DVCS number of events should be scaled is not known.

If we assume the chance of missing an event was independent of event kinematics, the same DVCS cross section fitting procedure can be performed by scaling the number of events in each bin by 106-109\% (and 100\%/no scaling for Kin-481). The $\chi^{2}$ from the resulting fit, are given in table 6.5, alongside the results from the original fit. According to the $\chi^{2}$ results with the DIS-motivated scaling, the quality of the cross section fitting is worse when the number of events are scaled in each bin.

\begin{tabular}{|c|c|c|c|c|}
\hline Kin Setting & $\chi_{\text {unpol }}^{2} / \operatorname{dof}$ & $\chi_{\text {pol }}^{2} /$ dof & $\chi_{\text {unpol }}^{2} /$ dof (scaled) & $\chi_{\text {pol }}^{2} /$ dof (scaled) \\
\hline 482 & 1.35 & 0.94 & 1.62 & 0.95 \\
483 & 1.68 & 0.95 & 2.44 & 0.97 \\
484 & 1.39 & 1.02 & 1.74 & 1.13 \\
\hline
\end{tabular}

Table 6.5: The normalized $\chi^{2}$ for the $x_{B}=0.48$ kinematic settings with the DIS-motivated scaling, compared to without.

The values of the extracted cross section in each bin are similar with and without the DIS-motivated scaling. The results with the DIS-motivated scaling and are shown for the unpolarized case in figures 6.10, 6.12, and 6.14 for reference. The same figures without scaling are shown for each kinematic in figures 6.9, 6.11, and 6.13. The values given in the tables of results in the appendices, are given without the scaling discussed in this section. The extracted CFF values with and without this scaling are discussed in the following section 6.4. 

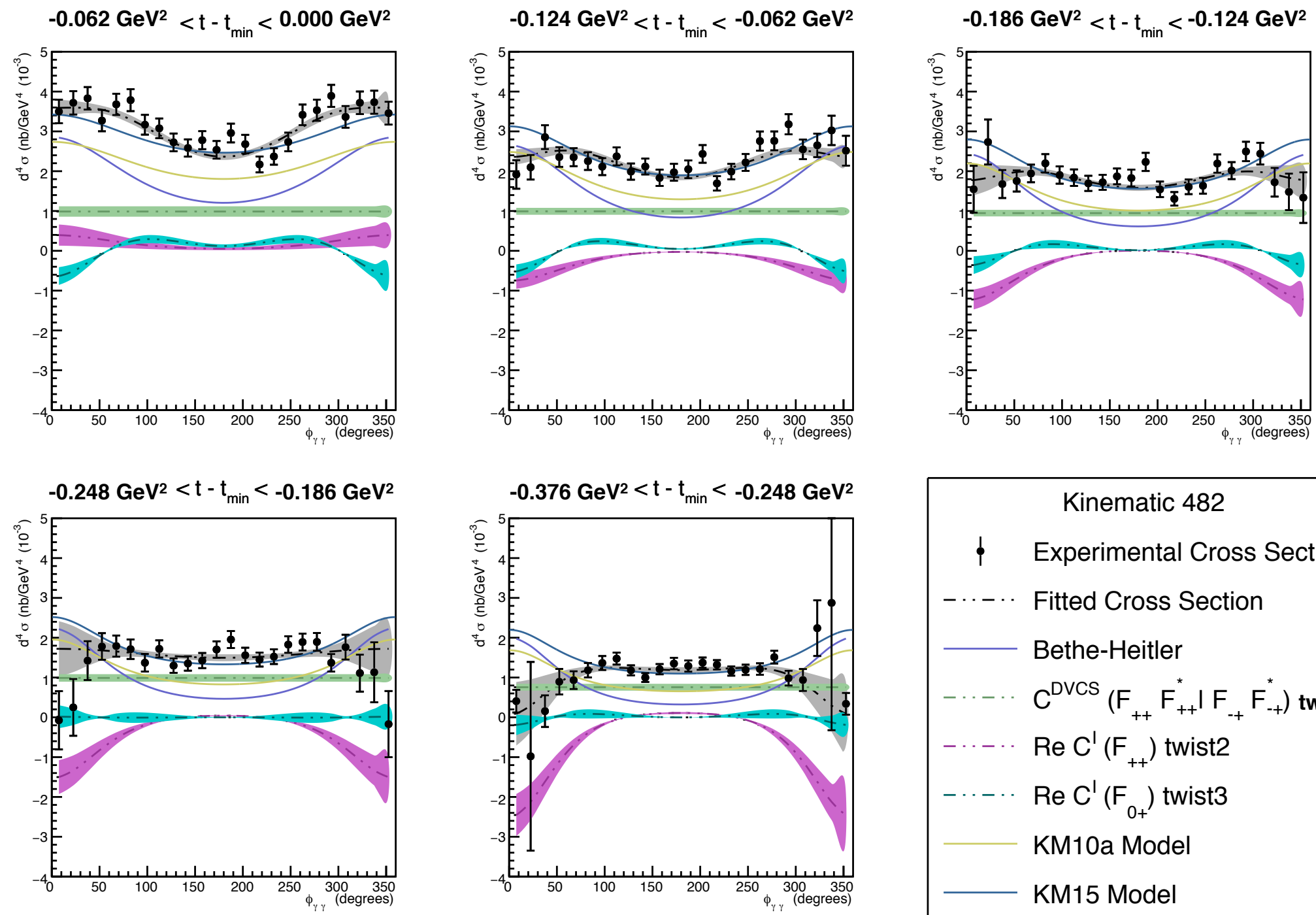

\begin{tabular}{|c|c|}
\hline & Kinematic 482 \\
\hline$\phi$ & Experimental Cross Section \\
\hline$-\cdots-\cdots$ & Fitted Cross Section \\
\hline & Bethe-Heitler \\
\hline$-\cdots-$ & $\mathrm{C}^{\text {DVCS }}\left(\mathrm{F}_{++} \mathrm{F}_{++}^{*} \mid \mathrm{F}_{-+} \mathrm{F}_{-+}^{*}\right)$ twist? \\
\hline$-\cdots-$ & $\operatorname{Re} C^{\prime}\left(F_{++}\right)$twist2 \\
\hline$-\cdots-\cdots$ & $\operatorname{Re} C^{\prime}\left(F_{0+}\right)$ twist3 \\
\hline & KM10a Model \\
\hline & KM15 Model \\
\hline
\end{tabular}

Figure 6.9: The Kin 482 unpolarized fit results without any DIS-motivated scaling. 

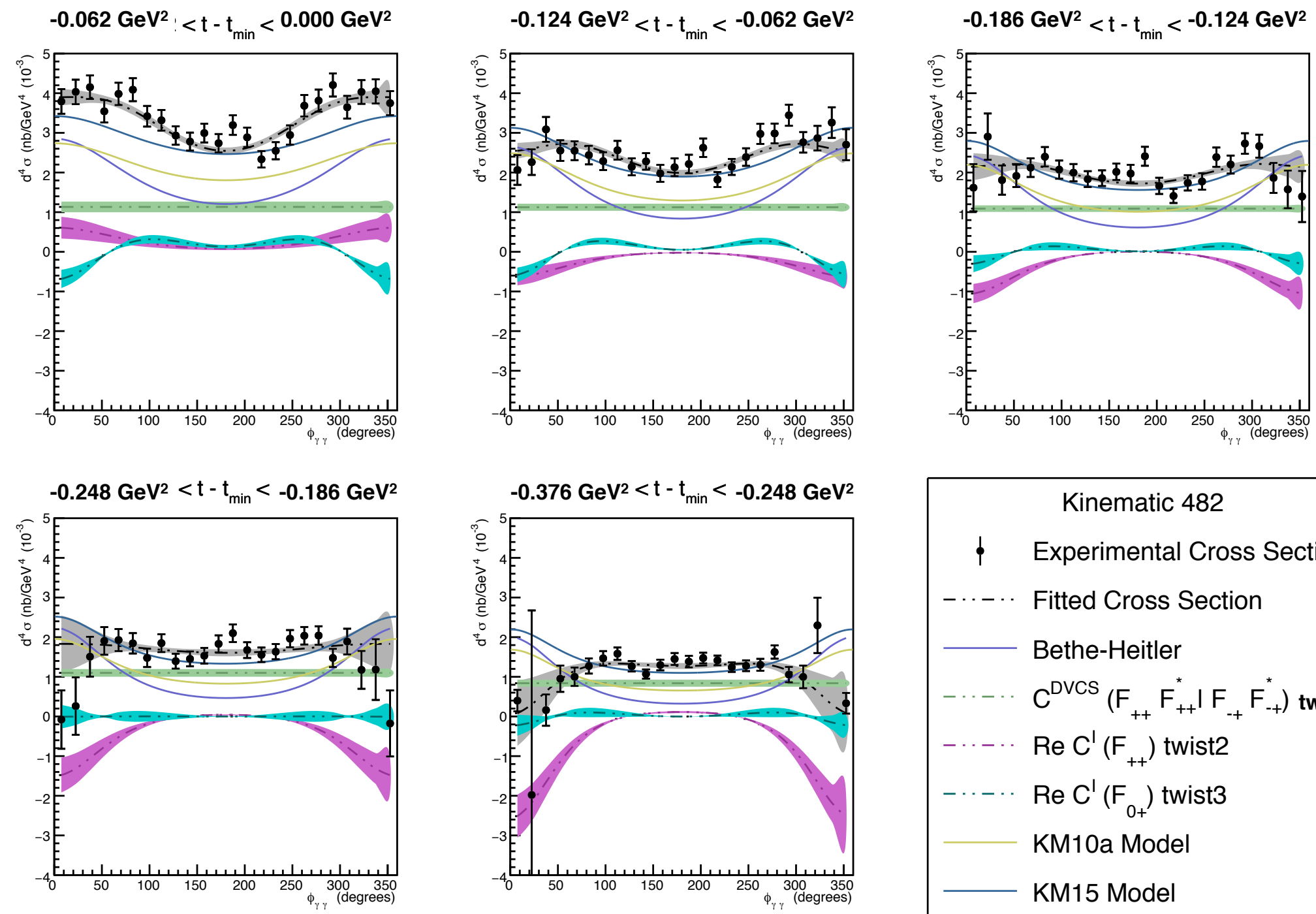

\begin{tabular}{|c|c|}
\hline & Kinematic 482 \\
\hline$\phi$ & Experimental Cross Section \\
\hline$-\cdots-$ & Fitted Cross Section \\
\hline & Bethe-Heitler \\
\hline$-\cdots-$ & $C^{\text {DVCS }}\left(F_{++} F_{++}^{*} \mid F_{-+} F_{-+}^{*}\right)$ twist2 \\
\hline$-\cdots-$ & $\operatorname{Re} C^{\prime}\left(F_{++}\right)$twist2 \\
\hline$-\cdots-$ & $\operatorname{Re} C^{\prime}\left(F_{0+}\right)$ twist3 \\
\hline & KM10a Model \\
\hline 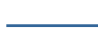 & KM15 Model \\
\hline
\end{tabular}

Figure 6.10: The Kin 482 unpolarized fit results with the $106 \%$ DIS-motivated scaling. See figure 6.9 above for the result without scaling. 

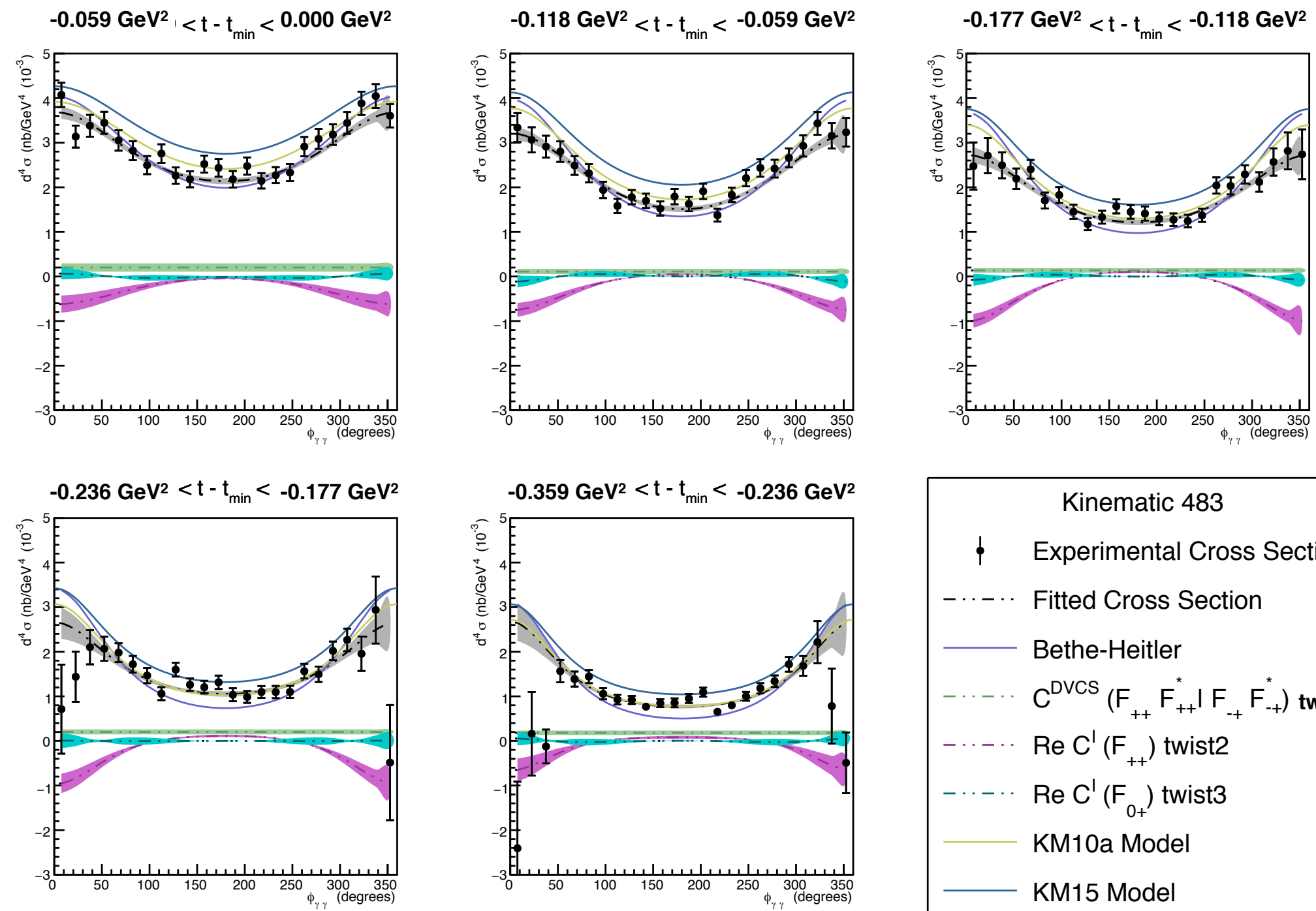

Kinematic 483

$\downarrow \quad$ Experimental Cross Section

-... F Fitted Cross Section

Bethe-Heitler $\mathrm{C}^{\mathrm{DVCS}}\left(\mathrm{F}_{++} \mathrm{F}_{++}^{*} \mathrm{I} \mathrm{F}_{-+} \mathrm{F}_{-+}^{*}\right)$ twist2

$-\cdots-\cdots \operatorname{Re} C^{\prime}\left(F_{++}\right)$twist2

- . - $\operatorname{Re} C^{\prime}\left(\mathrm{F}_{0+}\right)$ twist3

KM10a Model

KM15 Model

Figure 6.11: The Kin 483 unpolarized fit results without any DIS-motivated scaling. 

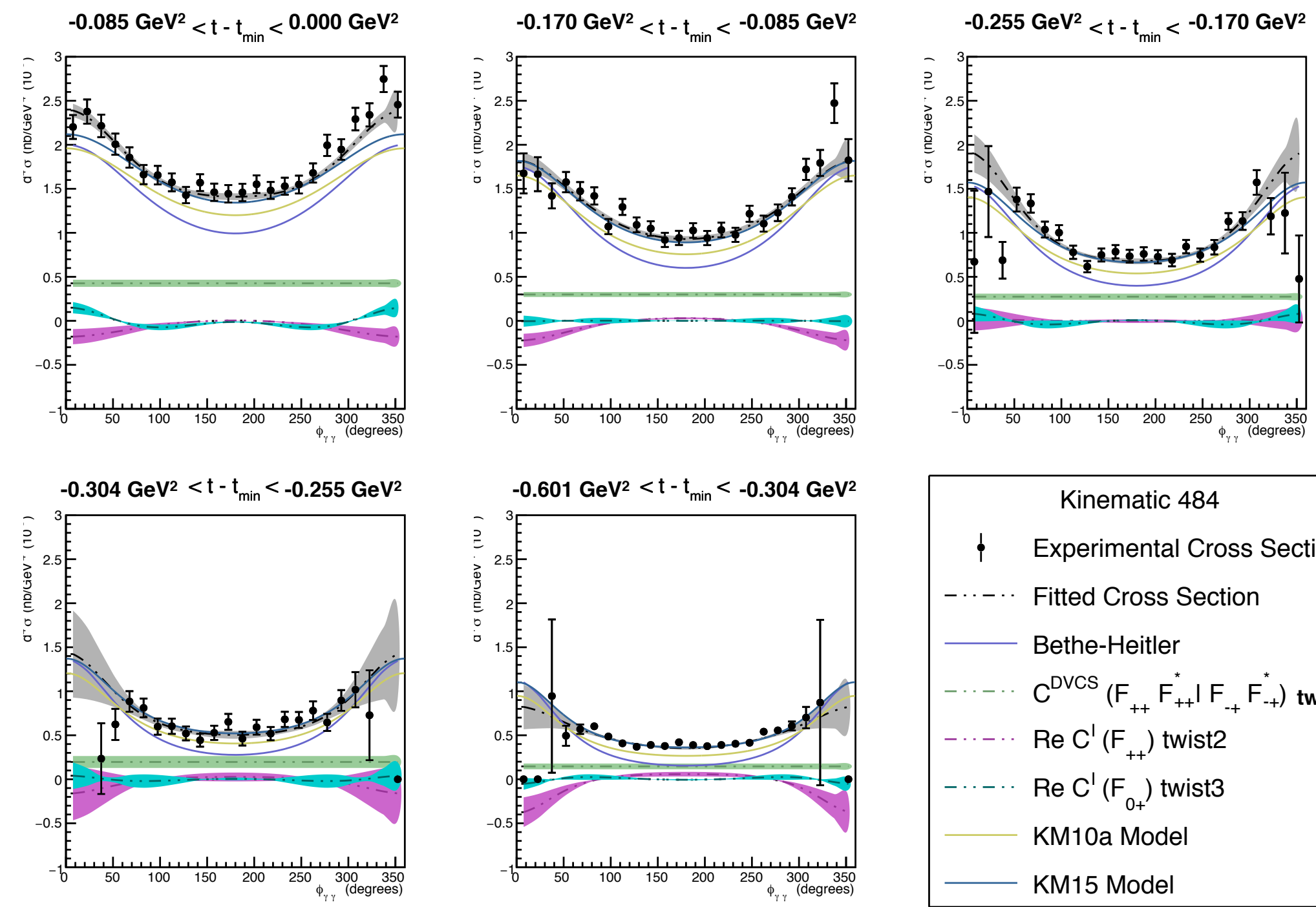

\begin{tabular}{|c|c|}
\hline & Kinematic 484 \\
\hline$\phi$ & Experimental Cross Section \\
\hline$-\cdots-$ & Fitted Cross Section \\
\hline & Bethe-Heitler \\
\hline & $C^{\text {DVCS }}\left(F_{++} F_{++}^{*} \mid F_{-+} F_{-+}^{*}\right)$ twist \\
\hline$-\cdots-$ & $\operatorname{Re} C^{\prime}\left(F_{++}\right)$twist2 \\
\hline$-\cdots-$ & $\operatorname{Re} C^{\prime}\left(F_{0+}\right)$ twist3 \\
\hline & KM10a Model \\
\hline & KM15 Model \\
\hline
\end{tabular}

Figure 6.13: The Kin 484 unpolarized fit results without any DIS-motivated scaling. 
independent of $Q^{2}$. Note that $\mathcal{F}_{0+}$ does have a $Q^{2}$ dependence according to equation 1.39 , but we expect this term to be nearly zero for all $Q^{2}$ if the handbag diagram dominates the cross section. Finally, the extracted $\mathcal{C}^{D V C S}\left(\mathcal{F}_{++} \mathcal{F}_{++}^{*} \mid \mathcal{F}_{+-} \mathcal{F}_{+-}^{*}\right)$ has contributions from both $\mathcal{F}_{++}$and $\mathcal{F}_{+-}$and its $Q^{2}$ dependence may not be straightforward to interpret.

If twist-4+ contributions were present in the data, but not in our parameterization, we would expect to see a $Q^{2}$ dependence in our results. The CFFCs, $X_{n}$ from the fitting procedure, described in section 6.2, were extracted in each of the five vertex $t$-bins for the $x_{B}=0.48$ kinematic settings. The $t$-bins were chosen so the bins for all $x_{B}=0.48$ results had similar values of $t$.

The resulting CFFCs for kinematics 481, 482, 483, and 484 are studied. The lighter set of data on each plot are the results after scaling the number of events in each bin by the DIS-motivated value $(100 \%, 106 \%, 106 \%$, and $109 \%$ for these kinematic settings in order). Note that the vertical axis in each plot is unit-less.

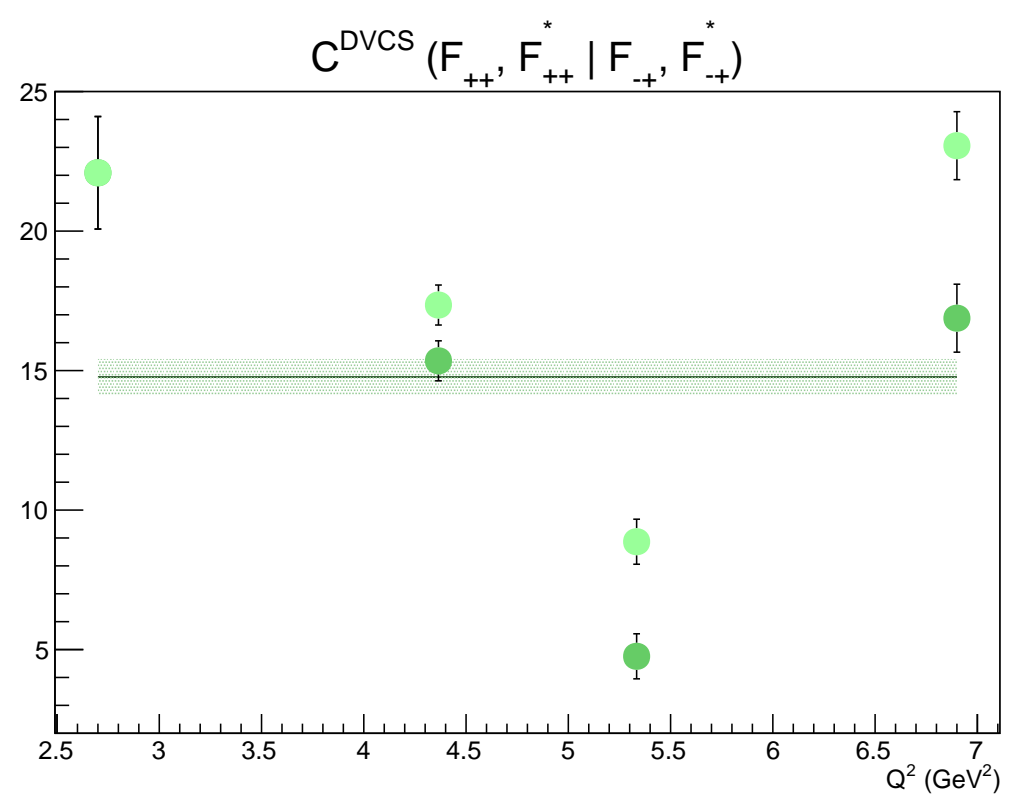

Figure 6.16: $Q^{2}$ dependence of the extracted twist-2 $\mathcal{C}^{D V C S}\left(\mathcal{F}_{++} \mathcal{F}_{++}^{*} \mid \mathcal{F}_{-+} \mathcal{F}_{-+}^{*}\right)$ term. Each data point has been averaged over the five vertex $t$-bins from the respective kinematic setting. The error bars are statistical. The horizontal line and corresponding uncertainty band represent the average of the 4 data points, and the propagated error associated with the average. The data points from left to right are from Kin 481, 482, 483 and 484.

Figures 6.16 and 6.15 show the $Q^{2}$ dependence of the twist-2 $\mathcal{C}^{D V C S}\left(\mathcal{F}_{++} \mathcal{F}_{++}^{*} \mid \mathcal{F}_{-+} \mathcal{F}_{-+}^{*}\right)$. The first figure shows the results in all 5 t-bins for all four Kin-48 settings, while the second figure shows the averaged value for each kinematic setting.

The result of the test for this term is inconclusive. The results presented in this figure may show an indication of $Q^{2}$ independence, but the results for the same $x_{B}=0.48$ data in Frederic Georges' analysis [63] show a distinct downward trend as $Q^{2}$ increases. However, 


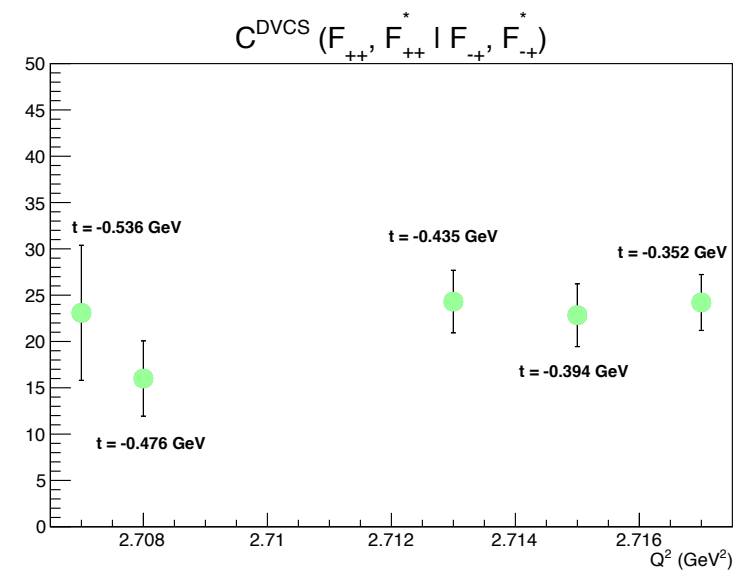

(a) Kin 481

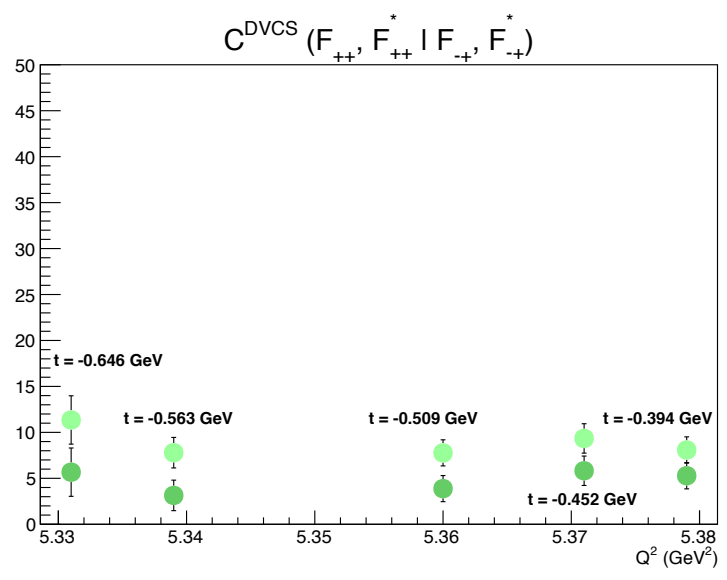

(c) Kin 483

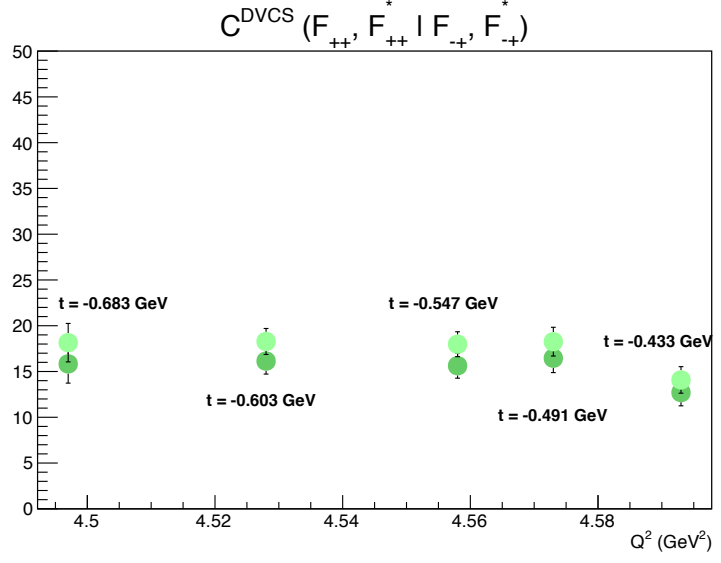

(b) Kin 482

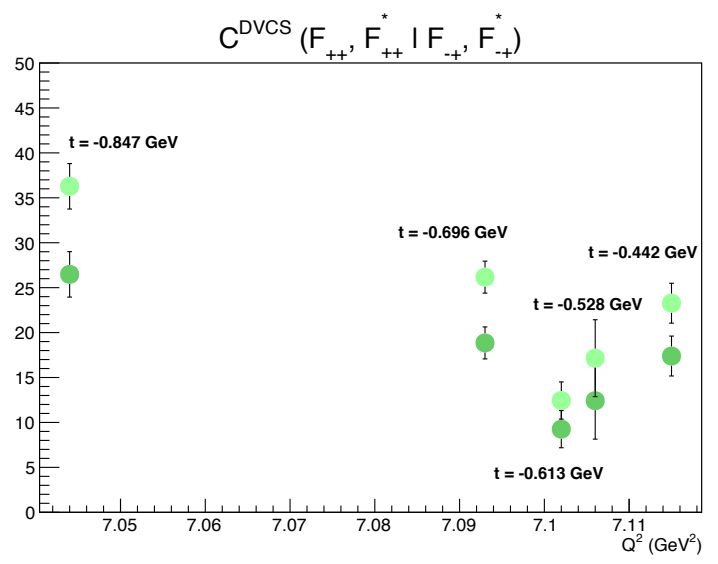

(d) Kin 484

Figure 6.15: The $\mathcal{C}^{D V C S}\left(\mathcal{F}_{++} \mathcal{F}_{++}^{*} \mid \mathcal{F}_{+-} \mathcal{F}_{+-}^{*}\right)$ CFFC extracted in all 5 t-bins for each kinematic setting, as a function of $Q^{2}$. The error bars are the resulting error from the fitting procedure. The dark (green) data points are the resulting values from fitting the data without any corrections related to the DIS cross section extraction results. The light (green) data points are the results after scaling the number of events in each bin by the DIS-motivated value $(100 \%, 106 \%, 106 \%$, and $109 \%$ for these kinematic settings in order). 
Frederic's results also do show an indication of $Q^{2}$ independence for this CFF in the $x_{B}=$ 0.36 and $x_{B}=0.60$ data. For comparison, $Q^{2}$ independence was observed for this term in the $x_{B}=0.36$ results seen in a past DVCS experiment in Hall A [65].

It is important to note that this CFFC also includes contributions from the gluon transversity CFFs $\mathcal{F}_{+-} \approx \mathcal{F}_{T}$. Consequently the interpretation of the scaling of the $\mathcal{C}^{D V C S}\left(\mathcal{F}_{++} \mathcal{F}_{++}^{*} \mid \mathcal{F}_{-+} \mathcal{F}_{-+}^{*}\right)$ term may not be straightforward.

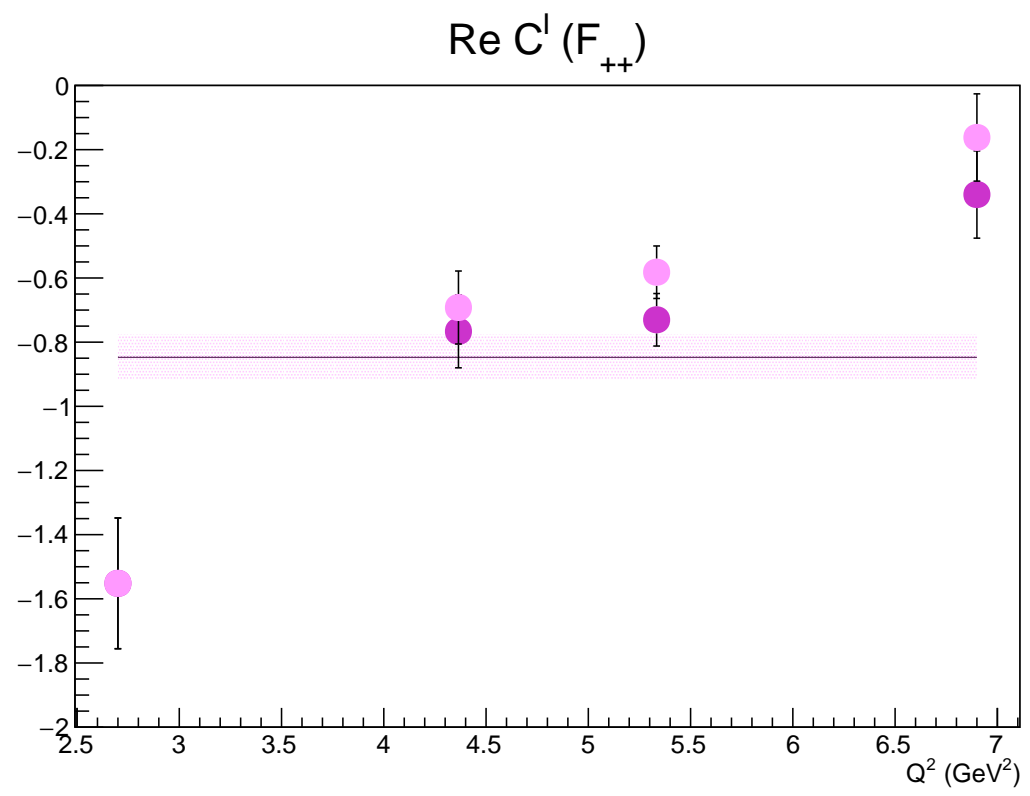

Figure 6.18: $Q^{2}$ dependence of the extracted twist-2 $\operatorname{Re} \mathcal{C}^{I}\left(\mathcal{F}_{++}\right)$CFFC. Each data point has been averaged over the five vertex $t$-bins from the respective kinematic setting. The error bars are statistical. The horizontal line and corresponding uncertainty band represent the average of the 4 data points, and the propagated error associated with the average. The data points from left to right are from Kin 481, 482, 483 and 484.

Figures 6.18 and 6.17 show the $Q^{2}$ dependence of the twist-2 $\operatorname{Re} \mathcal{C}^{I}\left(\mathcal{F}_{++}\right)$term. The first figure shows the results in all 5 t-bins for all four Kin- 48 settings, while the second figure shows the averaged value for each kinematic setting.

The result of the test for this term is also inconclusive. The results presented in this figure may show an indication of $Q^{2}$ independence. Data taken at $Q^{2}<2.5 \mathrm{GeV}^{2}$ or $Q^{2}>$ $7 \mathrm{GeV}^{2}$ could help determine if there is an upward trend as $Q^{2}$ increases. The results found by Frederic Georges [63] in his analysis of the same data show a more distinct upward trend, which is not apparent in the $x_{B}=0.36$ and $x_{B}=0.60$ kinematic settings. For comparison, $Q^{2}$ independence was observed in the $x_{B}=0.36$ scaling test seen in a past DVCS experiment in Hall A 65]. 


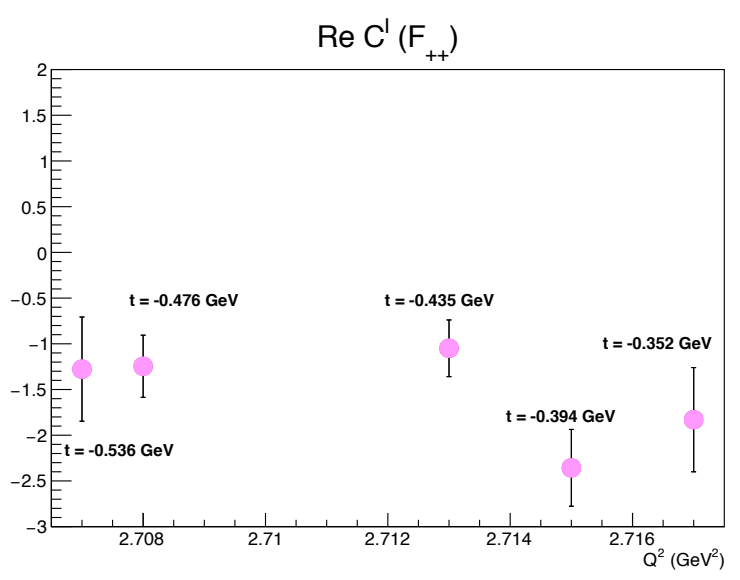

(a) Kin 481

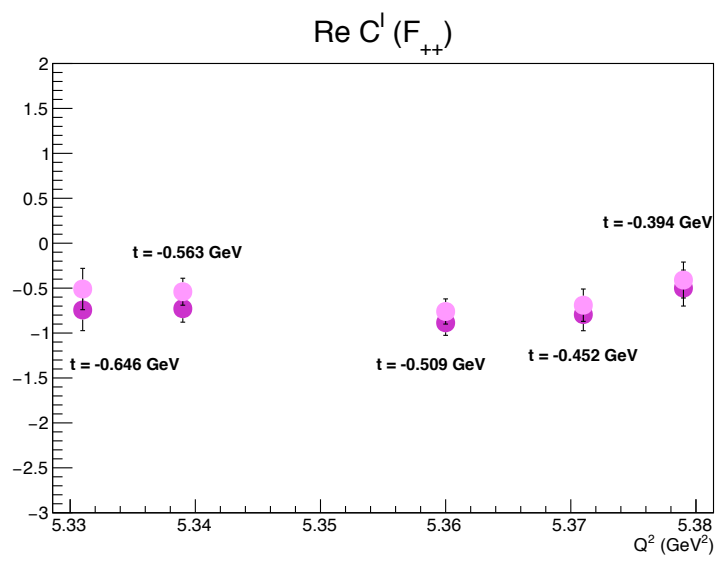

(c) Kin 483

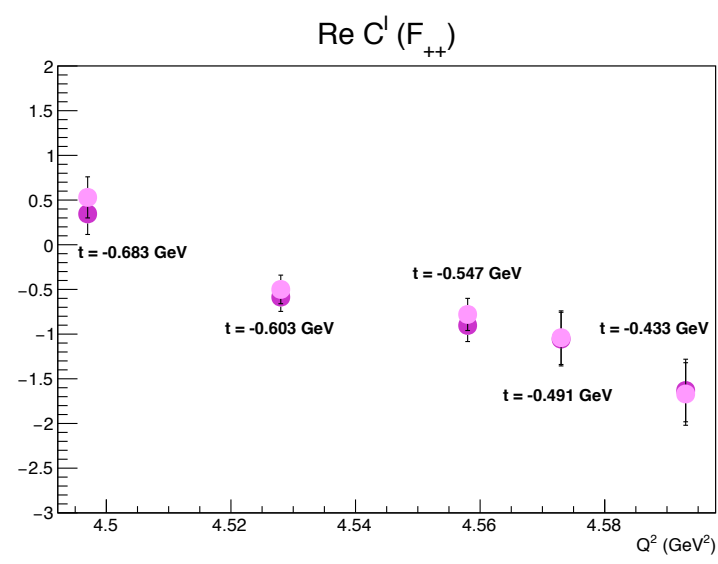

(b) Kin 482

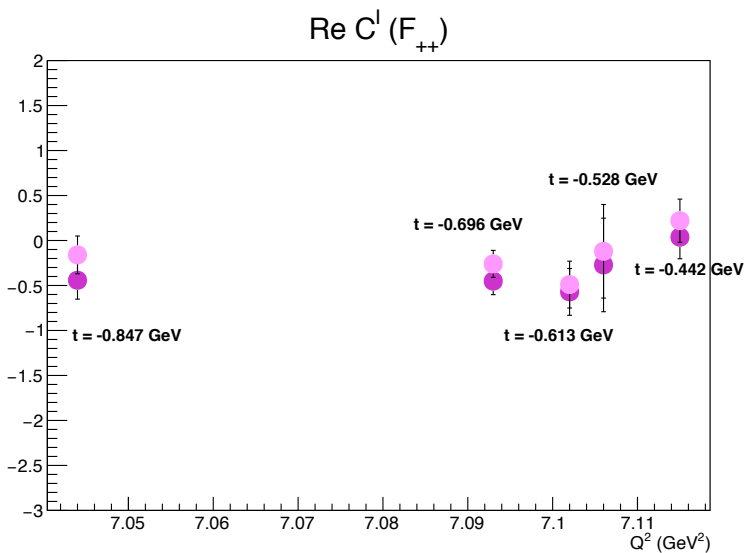

(d) Kin 484

Figure 6.17: The $\operatorname{Re} \mathcal{C}^{I}\left(\mathcal{F}_{++}\right)$CFFC extracted in all 5 t-bins for each kinematic setting, as a function of $Q^{2}$. The error bars are the resulting error from the fitting procedure. The dark (magenta) data points are the resulting values from fitting the data without any corrections related to the DIS cross section extraction results. The light (magenta) data points are the results after scaling the number of events in each bin by the DIS-motivated value (100\%, $106 \%, 106 \%$, and $109 \%$ for these kinematic settings in order). 


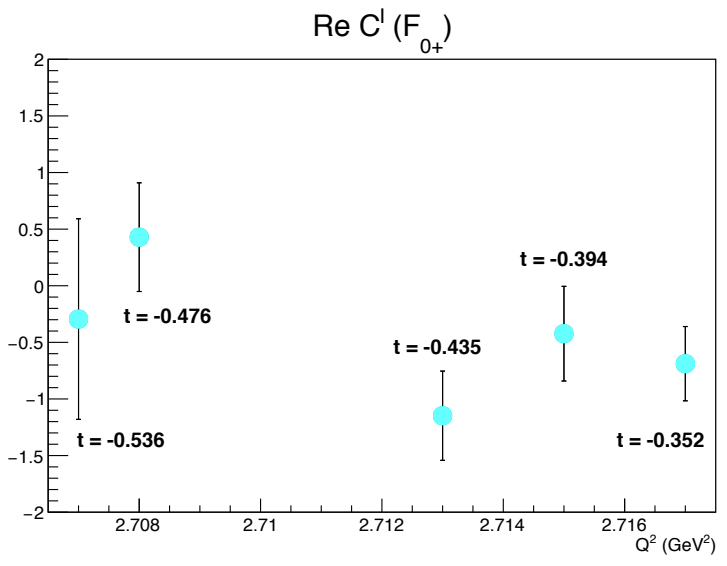

(a) Kin 481

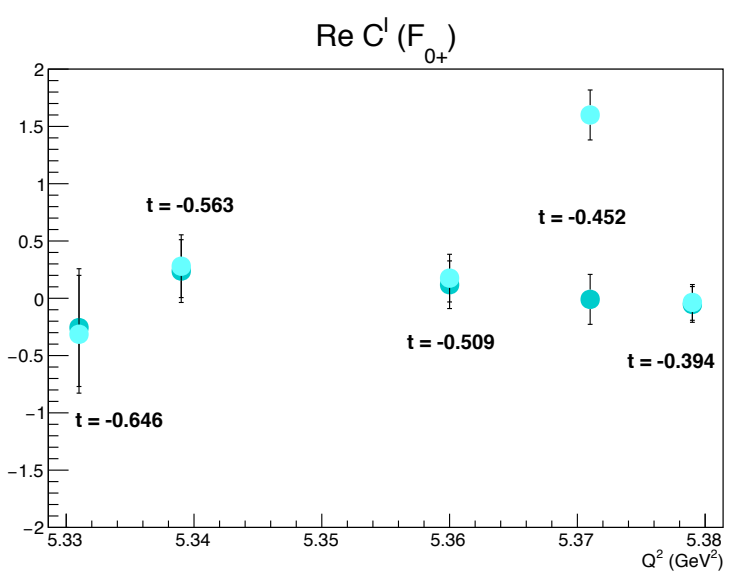

(c) Kin 483

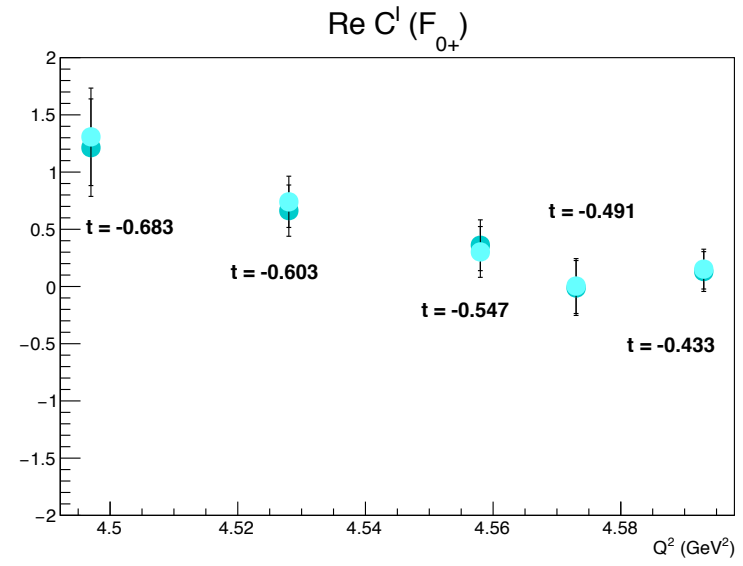

(b) Kin 482

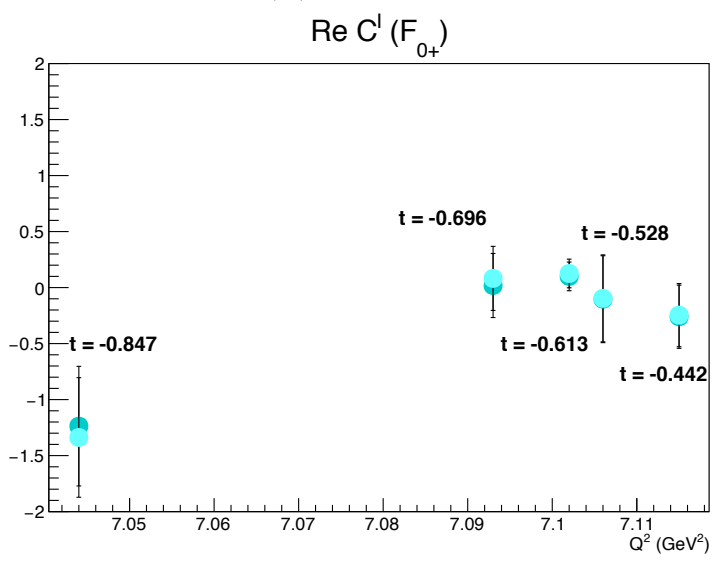

(d) Kin 484

Figure 6.19: The $\operatorname{Re} \mathcal{C}^{I}\left(\mathcal{F}_{0+}\right)$ CFFC extracted in all 5 t-bins for each kinematic setting, plotted vs. $Q^{2}$. The error bars are the resulting error from the fitting procedure. The dark (cyan) data points are the resulting values from fitting the data without any corrections related to the DIS cross section extraction results. The light (cyan) data points are the results after scaling the number of events in each bin by the DIS-motivated value (100\%, $106 \%, 106 \%$, and 109\% for these kinematic settings in order). 


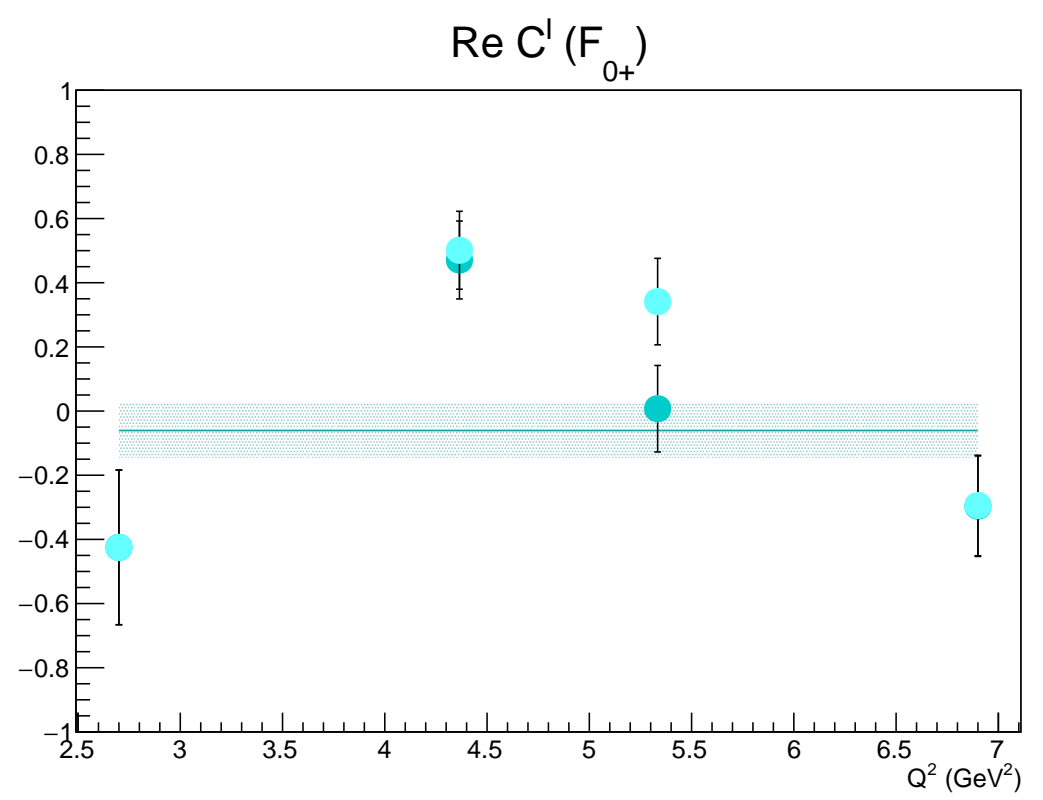

Figure 6.20: The extracted twist-3 Re $\mathcal{C}^{I}\left(\mathcal{F}_{0+}\right)$ CFFC. Each data point has been averaged over the five vertex $t$-bins from the respective kinematic setting. The error bars are statistical. The horizontal line and corresponding uncertainty band represent the average of the 4 data points, and the propagated error associated with the average. The data points from left to right are from Kin 481, 482, 483 and 484.

Figures 6.20 and 6.19 show the twist-3 $\operatorname{Re} \mathcal{C}^{I}\left(\mathcal{F}_{0+}\right)$. The first figure shows the results in all 5 t-bins for all four Kin-48 settings, while the second figure shows the averaged value for each kinematic setting. The result presented in this figure shows that this term is very close to zero for all $Q^{2}$. This is consistent with the $x_{B}=0.36$ results seen in a past DVCS experiment in Hall A [65]. 


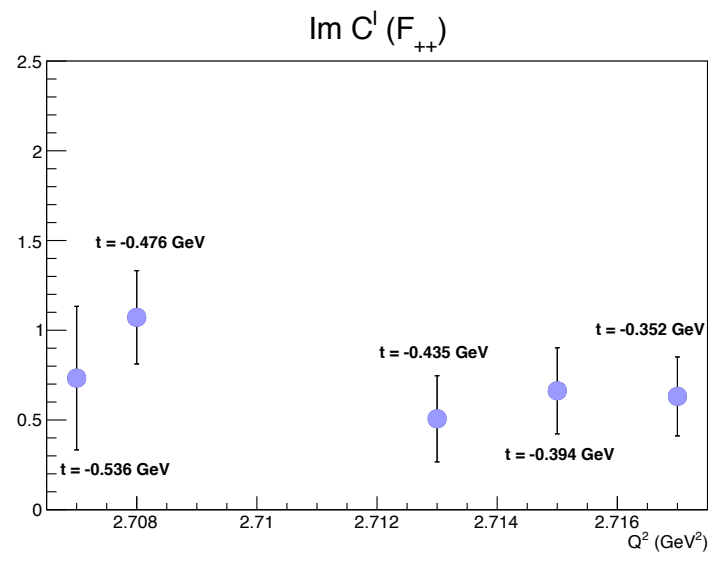

(a) Kin 481

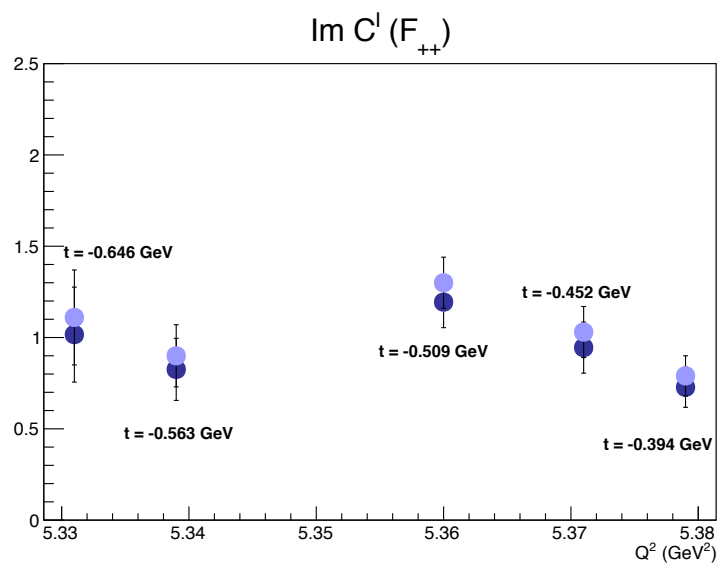

(c) Kin 483

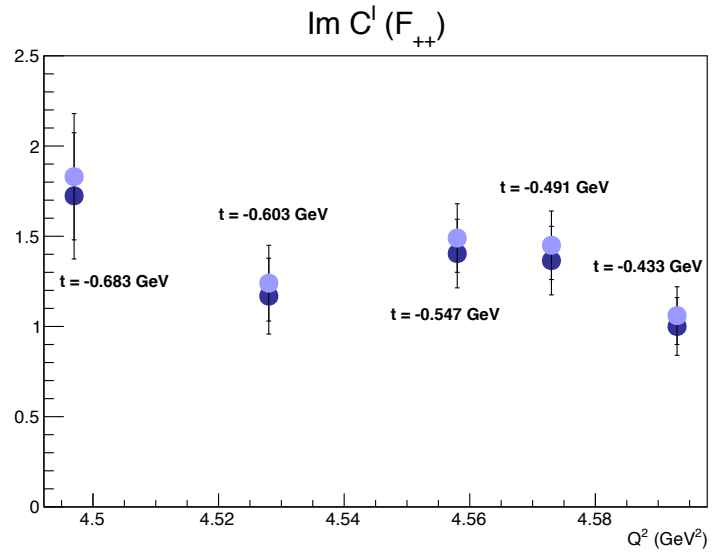

(b) Kin 482

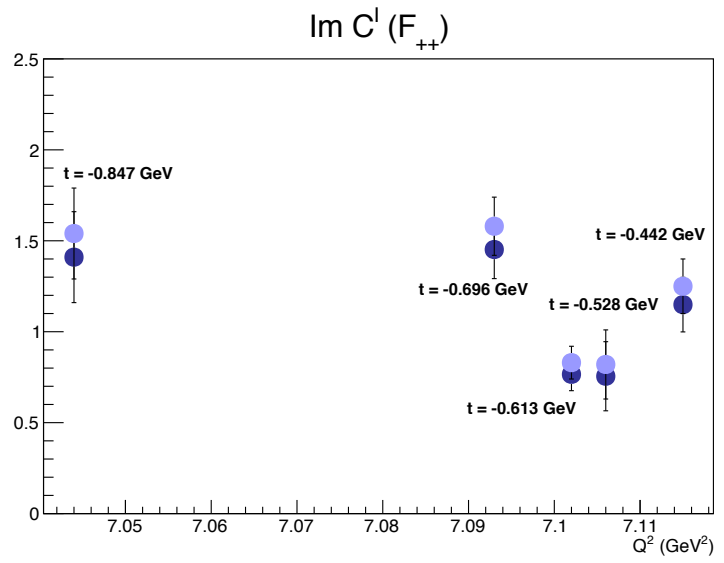

(d) Kin 484

Figure 6.21: The $\operatorname{Im} \mathcal{C}^{I}\left(\mathcal{F}_{++}\right)$CFFC extracted in all 5 t-bins for each kinematic setting, as a function of $Q^{2}$. The error bars are the resulting error from the fitting procedure. The dark (blue) data points are the resulting values from fitting the data without any corrections related to the DIS cross section extraction results. The light (blue) data points are the results after scaling the number of events in each bin by the DIS-motivated value (100\%, $106 \%, 106 \%$, and $109 \%$ for these kinematic settings in order). 


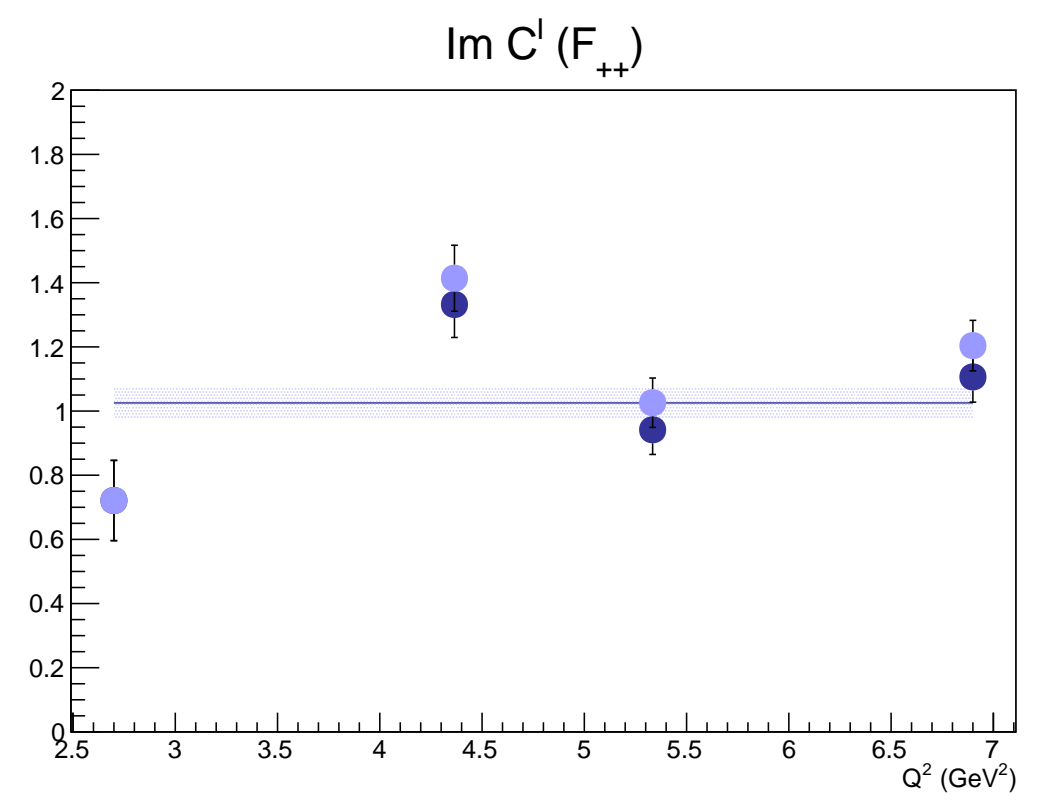

Figure 6.22: $Q^{2}$ dependence of the extracted twist-2 $\operatorname{Im} \mathcal{C}^{I}\left(\mathcal{F}_{++}\right)$CFFC. Each data point has been averaged over the five vertex $t$-bins from the respective kinematic setting. The error bars are statistical. The horizontal line and corresponding uncertainty band represent the average of the 4 data points, and the propagated error associated with the average. The data points from left to right are from Kin 481, 482, 483 and 484.

Figures 6.22 and 6.21 shows the $Q^{2}$ dependence test for the twist-2 $\operatorname{Im} \mathcal{C}^{I}\left(\mathcal{F}_{++}\right)$. The first figure shows the results in all 5 t-bins for all four Kin- 48 settings, while the second figure shows the averaged value for each kinematic setting. The result presented in this figure shows a good indication of $Q^{2}$ independence, and also that this term is non-zero. This result is consistent with that seen by Frederic Georges [63] and also a past DVCS experiment 65] in Hall A. 


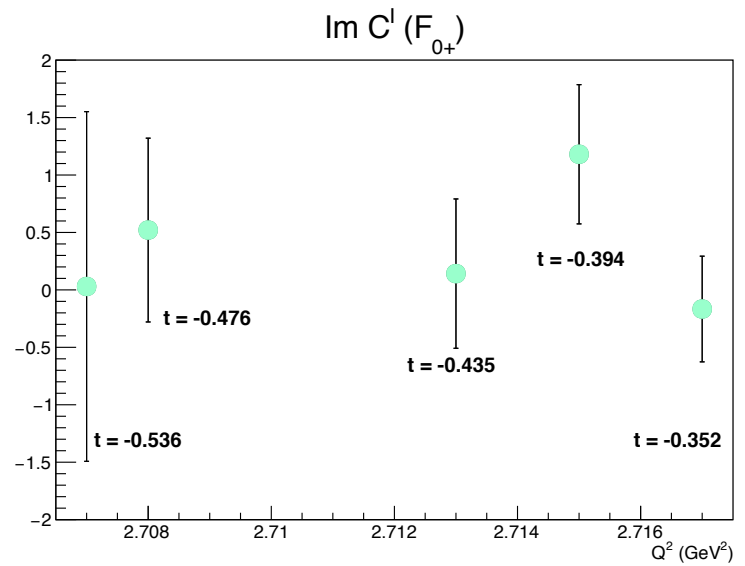

(a) Kin 481

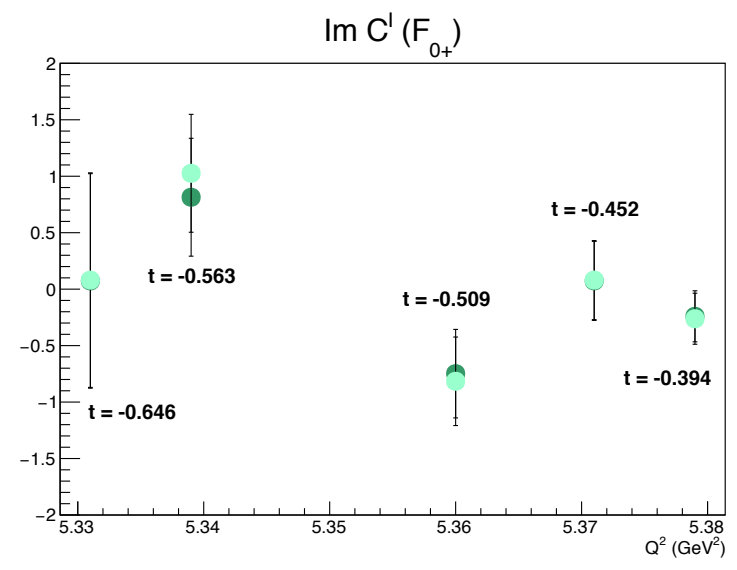

(c) Kin 483

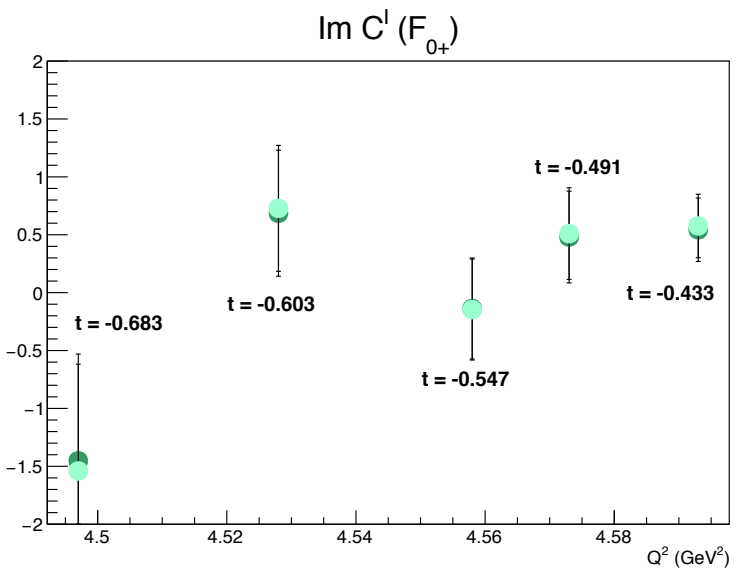

(b) Kin 482

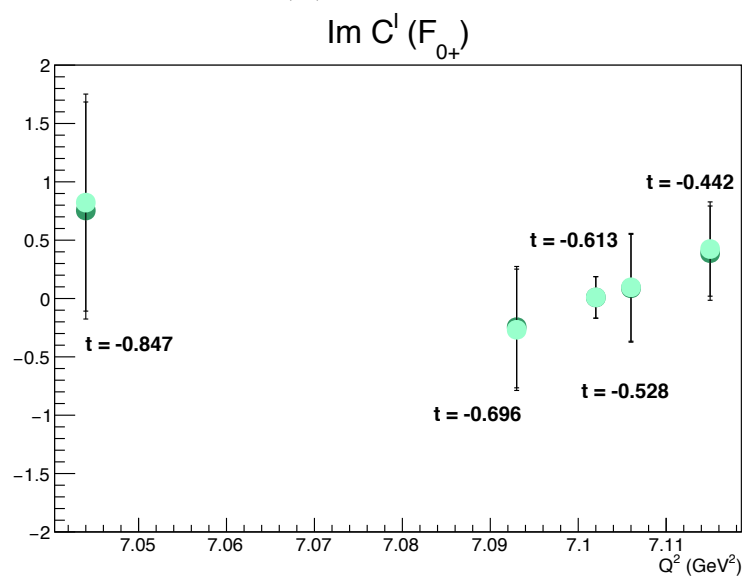

(d) Kin 484

Figure 6.23: The $\operatorname{Im} \mathcal{C}^{I}\left(\mathcal{F}_{0+}\right)$ CFFC extracted in all 5 t-bins for each kinematic setting, plotted vs. $Q^{2}$. The error bars are the resulting error from the fitting procedure. The dark (aqua) data points are the resulting values from fitting the data without any corrections related to the DIS cross section extraction results. The light (aqua) data points are the results after scaling the number of events in each bin by the DIS-motivated value (100\%, $106 \%, 106 \%$, and 109\% for these kinematic settings in order). 


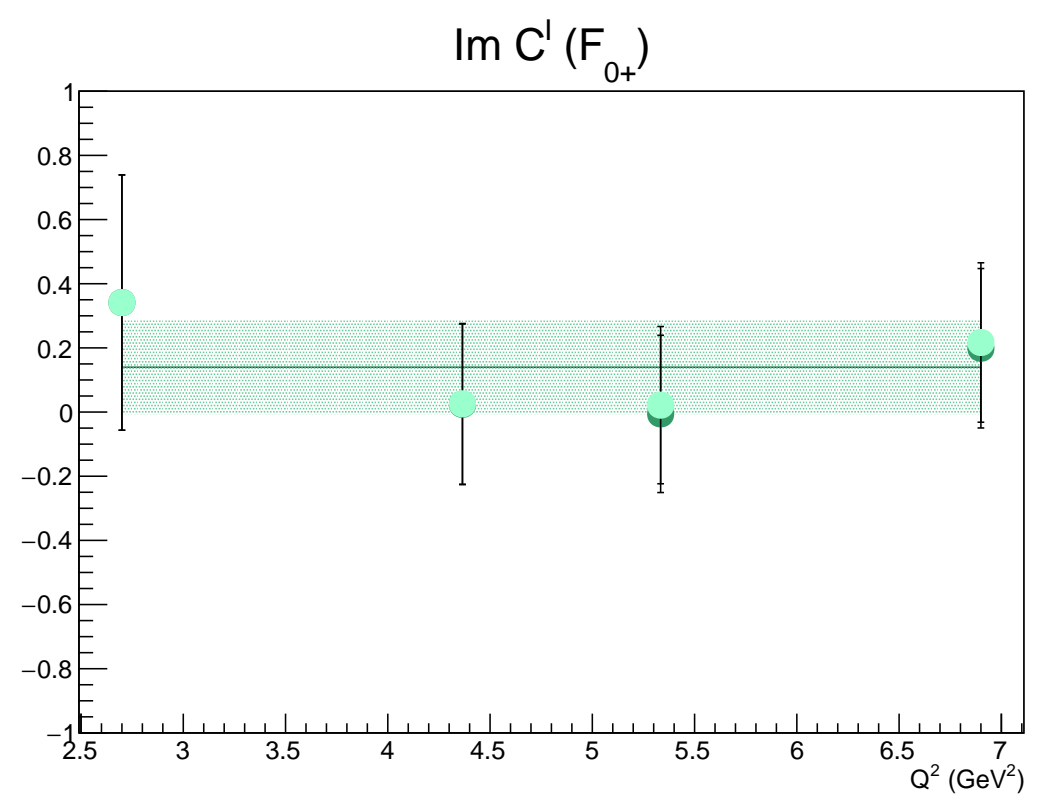

Figure 6.24: $Q^{2}$ dependence of the extracted twist-3 $\operatorname{Im} \mathcal{C}^{I}\left(\mathcal{F}_{0+}\right)$ CFFC. Each data point has been averaged over the five vertex $t$-bins from the respected kinematic setting. The error bars are statistical. The horizontal line and corresponding uncertainty band represent the average of the 4 data points, and the propagated error associated with the average. The data points from left to right are from Kin 481, 482, 483 and 484.

Figures 6.24 and 6.23 show the twist-3 $\operatorname{Im} \mathcal{C}^{I}\left(\mathcal{F}_{0+}\right)$. The first figure shows the results in all 5 t-bins for all four Kin-48 settings, while the second figure shows the averaged value for each kinematic setting. The result presented in this figure shows that this term is very close to zero for all $Q^{2}$. Note that even though this term is very close to zero, it may not actually equal zero, indicating the possibility of twist-3 contributions to the DVCS cross section. This result is consistent with that seen by Frederic Georges [63] and also a past DVCS experiment in Hall A 65.

Table 6.6 summarizes the mean values of the CFFCs extracted in this analysis.

\begin{tabular}{|l|c|c|c|c|}
\hline CFFC & Mean Value & Error & Mean Value (w/ DIS-Motivated Scaling) & Error \\
\hline$C^{D V C S}\left(\mathcal{F}_{++}, \mathcal{F}_{++}^{*} \mid \mathcal{F}_{-+}, \mathcal{F}_{-+}^{*}\right)$ & 14.77 & \pm 0.65 & 17.84 & \pm 0.65 \\
$\mathcal{R} e C^{I}\left(\mathcal{F}_{++}\right)$ & -0.85 & \pm 0.07 & -0.75 & \pm 0.07 \\
$\mathcal{R} e C^{I}\left(\mathcal{F}_{0+}\right)$ & -0.03 & \pm 0.08 & 0.02 & \pm 0.09 \\
$\mathcal{I} m C^{I}\left(\mathcal{F}_{++}\right)$ & 1.03 & \pm 0.05 & 1.09 & \pm 0.06 \\
$\mathcal{I} m C^{I}\left(\mathcal{F}_{0+}\right)$ & 0.09 & \pm 0.15 & 0.10 & \pm 0.15 \\
\hline
\end{tabular}

Table 6.6: Mean CFFCs values extracted from the fit of the polarized and unpolarized DVCS cross sections. The errors listed here are the propagated error associated with the average, and not the standard deviation of the average. 


\section{Chapter 7}

\section{Conclusion}

The E12-06-114 experiment ran in Hall A at Jefferson Lab in Fall 2014 and Spring and Fall 2016, running with three different $x_{B}$ values, each having 2-4 $Q^{2}$ values. The DVCS cross section is considered the golden channel for accessing GPDs, however the cross section is very small. The high luminosity capabilities afforded by the Hall A detectors allows for DVCS cross section extraction with high statistical precision. This experiment extended the kinematic regimes in which the DVCS cross section was extracted from that of the previous E00-110 Hall-A experiment.

The E12-06-114 experiment faced several technical challenges. This experiment was one of the first to run after the $12 \mathrm{GeV}$ accelerator upgrade, and the accelerator was unable to consistently provide beam to the hall. Because of this, nearly $50 \%$ of the planned PAC days were not completed, including two entire kinematic settings planned at $x_{B}=0.60$.

During Spring 2016, the L-HRS Q1 was could not reach the required current and was detuned for kinematic settings 482, 483, and 484. For Fall 2016 running, the magnet was replaced, but the replacement unexpectedly suffered saturation effects. Because of this, optics calibration needed to be performed for all kinematic settings individually, and new R-Functions were developed for each setting as a result.

The photon calorimeter suffered effects of radiation damage due to aging of the blocks, high luminosity, and high energy of the detected photons. The normal elastic calibration needs dedicated beam time and was not practical to be performed often. Instead, the $\pi^{0}$ calibration was performed nearly daily to compensate for the radiation damage in the calorimeter.

The DIS cross section was extracted for every kinematic setting (as described in section 4.2). For settings 361 and 481, the $Q 1$ was fully functioning and the extracted cross section agreed well with the expected cross section. However, for the settings where $Q 1$ was either saturated $(362,363,601,603)$ or detuned $(482,483,484)$, the extracted and expected

DIS cross sections disagreed up to $9 \%$. A parallel analysis performed by Bishnu Karki using a Geant 4 simulation [73], yielded a similar discrepancy. The reason for the discrepancy is still unknown, but it is suspected that the issue could due to an unknown effect related to the $Q 1$ functionality, or the presence of the S0\&Cer trigger for DIS data taking.

In this document, the DVCS cross section was extracted for the $x_{B}=0.48$ kinematic 
settings (section 6.2.7 and appendices B, D, and E). The extraction for the $x_{B}=0.36$ and $x_{B}=0.6$ settings was performed by both Frederic Georges [63] and Hashir Rashad (thesis pending). The extracted DVCS cross sections are observed to show good agreement with the KM15 model (appendix B).

The extracted $\operatorname{Im} \mathcal{C}^{I}\left(\mathcal{F}_{++}\right)$twist-2 CFFC was observed to show independence of $Q^{2}$ (figure 6.22), which is consistent with the result of the E00-110 experiment 65]. Unfortunately, the $Q^{2}$ independence observations for the twist-2 $\mathcal{C}^{D V C S}\left(\mathcal{F}_{++} \mathcal{F}_{++}^{*} \mid \mathcal{F}_{+-} \mathcal{F}_{+-}^{*}\right)$ and $\operatorname{Re} \mathcal{C}^{I}\left(\mathcal{F}_{++}\right)$ CFFCs were inconclusive (figures 6.16 and 6.18). This result may indicate a significant contribution from gluon CFFCs not completely accounted for in this analysis. Future DVCS experiments at Jefferson Lab are planned to separate CFFCs with the same $\phi_{\gamma \gamma}$ dependence. This will allow for the study of all CFFCs and their $Q^{2}$ independence, which may help in understanding these inclusive results.

The twist-3 CFFs are observed to be very close to zero, if not equal (figures 6.24 and 6.20). This result indicates the twist-2/handbag diagram dominance of the DVCS cross section. 


\section{Appendix A}

\section{Helicity CFFs and the DVCS Cross Section}

We are interested in extracting the helicity-conserving CFFs, $\left\{\mathcal{H}_{++}, \mathcal{E}_{++}, \tilde{\mathcal{H}}_{++}, \tilde{\mathcal{E}}_{++}\right\}$, single helicity flip CFFs $\left\{\mathcal{H}_{0+}, \mathcal{E}_{0+}, \tilde{\mathcal{H}}_{0+}, \tilde{\mathcal{E}}_{0+}\right\}$, and double helicity flip CFFs $\left\{\mathcal{H}_{-+}, \mathcal{E}_{-+}, \tilde{\mathcal{H}}_{-+}, \tilde{\mathcal{E}}_{-+}\right\}$ from section 1.3.1.

The CFFs are not extracted from the DVCS cross section individually, but rather in combinations according to the recent literature [17]. The possible combinations we can use to parameterize the cross section are:

\begin{tabular}{|l|c|c|c|}
\hline Combination & Twist & $\phi$ Dependence & Used in Fit \\
\hline $\mathcal{C}^{D V C S}\left(\mathcal{F}_{++}, \mathcal{F}_{++}^{*} \mid \mathcal{F}_{+-}, \mathcal{F}_{+-}^{*}\right)$ & twist-2 & constant & $\checkmark$ \\
\hline $\mathcal{C}^{D V C S}\left(\mathcal{F}_{0+}, \mathcal{F}_{0+}^{*}\right)$ & twist-3 & constant & \\
\hline $\operatorname{Re} \mathcal{C}^{I, V}\left(\mathcal{F}_{++}\right)$ & "twist-3" & constant & \\
\hline $\operatorname{Re} \mathcal{C}^{I}\left(\mathcal{F}_{++}\right)$ & twist-2 & $\cos \phi_{\gamma \gamma}$ & $\checkmark$ \\
\hline $\operatorname{Re} \mathcal{C}^{D V C S}\left(\mathcal{F}_{0+} \mid \mathcal{F}_{++}^{*}, \mathcal{F}_{+-}^{*}\right)$ & twist-3 & $\cos \phi_{\gamma \gamma}$ & \\
\hline $\operatorname{Re} \mathcal{C}^{I, A}\left(\mathcal{F}_{++}\right)$ & "twist-3" & $\cos \phi_{\gamma \gamma}$ & \\
\hline $\operatorname{Re} \mathcal{C}^{I}\left(\mathcal{F}_{0+}\right)$ & twist-3 & $\cos 2 \phi_{\gamma \gamma}$ & $\checkmark$ \\
\hline $\operatorname{Re} \mathcal{C}^{D V C S}\left(\mathcal{F}_{+-}, \mathcal{F}_{+-}^{*}\right)$ & twist-3 & $\cos 2 \phi_{\gamma \gamma}$ & \\
\hline $\operatorname{Im} \mathcal{C}^{I}\left(\mathcal{F}_{++}\right)$ & twist-2 & $\sin \phi_{\gamma \gamma}$ & $\checkmark$ \\
\hline $\operatorname{Im} \mathcal{C}^{D V C S}\left(\mathcal{F}_{0+} \mid \mathcal{F}_{++}^{*}, \mathcal{F}_{+-}^{*}\right)$ & twist-3 & $\sin \phi_{\gamma \gamma}$ & \\
\hline $\operatorname{Im} \mathcal{C}^{I, V}\left(\mathcal{F}_{++}\right)$ & "twist-3" & $\sin \phi_{\gamma \gamma}$ & \\
\hline $\operatorname{Im} \mathcal{C}^{I, A}\left(\mathcal{F}_{++}\right)$ & "twist-3" & $\sin \phi_{\gamma \gamma}$ & \\
\hline $\operatorname{Im} \mathcal{C}^{I}\left(\mathcal{F}_{0+}\right)$ & twist-3 & $\sin 2 \phi_{\gamma \gamma}$ & $\checkmark$ \\
\hline
\end{tabular}

Table A.1: A table of the CFFCs that can be used to parametrize the DVCS cross section. The notation $\mathcal{F}_{a b}$ follows from the helicity CFFs defined in section 1.3.1, $\mathcal{C}$ means combination, and the superscript DVCS and $I$ refer to CFFCs that contribute to the pure DVCS term and the BH-DVCS interference term, retrospectively. The superscripts $A$ and $V$ relate to the vector and axial vector functions from equation 1.17. More details on the $A$ and $V$ terms can be found in [17]. 
The quoted "twist-3" in the table means that the combination is twist-2, but kinematically suppressed like a twist-3. The expression for each combination, for an unpolarized target, in terms of the helicity $\mathrm{CFFs} \mathcal{F}_{a b}$ is given by 11 :

$$
\begin{gathered}
\mathcal{C}^{D V C S}\left(\mathcal{F}_{++}, \mathcal{F}_{++}^{*} \mid \mathcal{F}_{+-}, \mathcal{F}_{+-}^{*}\right)=\mathcal{C}^{D V C S}\left(\mathcal{F}_{++}, \mathcal{F}_{++}^{*}\right)+\mathcal{C}^{D V C S}\left(\mathcal{F}_{+-}, \mathcal{F}_{+-}^{*}\right) \\
\mathcal{C}^{D V C S}\left(\mathcal{F}_{0+} \mid \mathcal{F}_{++}^{*}, \mathcal{F}_{+-}^{*}\right)=\mathcal{C}^{D V C S}\left(\mathcal{F}_{0+}, \mathcal{F}_{++}^{*}\right)+\mathcal{C}^{D V C S}\left(\mathcal{F}_{0+}, \mathcal{F}_{+-}^{*}\right)
\end{gathered}
$$

where the combinations $\mathcal{C}^{D V C S}\left(\mathcal{F}_{a b}, \mathcal{F}_{c d}^{*}\right)$ are given by:

$$
\begin{gathered}
\mathcal{C}^{D V C S}\left(\mathcal{F}_{a b}, \mathcal{F}_{c d}^{*}\right)=\frac{4\left(1-x_{B}\right)\left(1+\frac{x_{B} t}{Q^{2}}\right)}{2-x_{B}+\frac{x_{B} t}{Q^{2}}}\left[\mathcal{H}_{a b} \mathcal{H}_{c d}^{*}+\tilde{\mathcal{H}}_{a b} \tilde{\mathcal{H}}_{c d}^{*}\right]+\frac{\left(2+\frac{t}{Q^{2}}\right) \epsilon^{2}}{\left(2-x_{B}+\frac{x_{B} t}{Q^{2}}\right)^{2}} \tilde{\mathcal{H}}_{a b} \tilde{\mathcal{H}}_{c d}^{*}-\frac{t}{4 M^{2}} \mathcal{E}_{a b} \mathcal{E}_{c d}^{*} \\
-\frac{x_{B}^{2}}{\left(2-x_{B}+\frac{x_{B} t}{Q^{2}}\right)^{2}}\left\{\left(1+\frac{t}{Q^{2}}\right)^{2}\left[\mathcal{H}_{a b} \mathcal{E}_{c d}^{*}+\mathcal{E}_{a b} \mathcal{H}_{c d}^{*}+\mathcal{E}_{a b} \mathcal{E}_{c d}^{*}\right]+\tilde{\mathcal{H}}_{a b} \tilde{\mathcal{E}}_{c d}^{*}+\tilde{\mathcal{E}}_{a b} \tilde{\mathcal{H}}_{c d}^{*}+\frac{t}{4 M^{2}} \tilde{\mathcal{E}}_{a b} \tilde{\mathcal{E}}_{c d}^{*}\right\} \\
\mathcal{C}^{I}\left(\mathcal{F}_{a b}\right)=F_{1} \mathcal{H}_{a b}-\frac{t}{4 M^{2}} F_{2} \mathcal{E}_{a b}+\frac{x_{B}}{2-x_{B}+\frac{x_{B} t}{Q^{2}}}\left(F_{1}+F_{2}\right) \tilde{\mathcal{H}}_{a b} \\
\mathcal{C}^{I, V}\left(\mathcal{F}_{a b}\right)=\frac{x_{B}}{2-x_{B}+\frac{x_{B} t}{Q^{2}}}\left(F_{1}+F_{2}\right)\left(\mathcal{H}_{a b}+\mathcal{E}_{a b}\right) \\
\mathcal{C}^{I, A}\left(\mathcal{F}_{a b}\right)=\frac{x_{B}}{2-x_{B}+\frac{x_{B} t}{Q^{2}}}\left(F_{1}+F_{2}\right) \tilde{\mathcal{H}}_{a b}
\end{gathered}
$$

where $F_{1}$ and $F_{2}$ are the Dirac and Pauli form factors, present because of the BH interference, and one can take the real or imaginary part of equations A.4, A.5, A.6.

When making the choice of which CFFCs to use in the parameterization, we choose only one term for each $\phi_{\gamma \gamma}$ dependence, so we can extract the single combination, instead of the sum of combinations. To extract the combinations individually from a sum, we would need experimental data at the same $\xi$ and $t$, but $n$ different beam energies or $Q^{2}$. Note that the $\phi_{\gamma \gamma}$ dependence introduced from the propagators $\mathcal{P}_{1}$ and $\mathcal{P}_{2}$ in the interference term is not enough to be able to separate the terms [66] 667.

\section{A.1 Definition of Variables}

For the variables used in the sections below, we have the following definitions:

$$
\tilde{K}=\frac{1}{Q} \sqrt{\left(1-x_{B}\right) x_{B}+\frac{\epsilon^{2}}{4}} \sqrt{\left(t_{\text {min }}-t\right)\left(t-t_{\text {max }}\right)}
$$

\footnotetext{
${ }^{1}$ Note that $\left|\frac{x_{B} t}{Q^{2}}\right|<<1$ in the E12-06-114 experiment
} 


$$
\begin{gathered}
K=\sqrt{1-y-\frac{\epsilon^{2}}{4} y^{2} \frac{\tilde{K}}{Q}} \\
t^{\prime}=t-t_{\text {min }} \\
t_{\text {min }}=-Q^{2} \frac{2\left(1-x_{B}\right)\left(1-\sqrt{1+\epsilon^{2}}\right)+\epsilon^{2}}{4 x_{B}\left(1-x_{B}\right)+\epsilon^{2}} \\
t_{\text {max }}=-Q^{2} \frac{2\left(1-x_{B}\right)\left(1+\sqrt{1+\epsilon^{2}}\right)+\epsilon^{2}}{4 x_{B}\left(1-x_{B}\right)+\epsilon^{2}}
\end{gathered}
$$

\section{A.2 The DVCS amplitude term}

The DVCS amplitude term is given by the expression:

$$
\left|\mathcal{T}_{D V C S}\right|^{2}=\frac{e^{6}}{y^{2} Q^{2}}\left[c_{0}^{D V C S}+\sum_{n=1}^{n=2}\left(c_{n}^{D V C S} \cos \left(n \phi_{\gamma \gamma}\right)+\lambda s_{n}^{D V C S} \sin \left(n \phi_{\gamma \gamma}\right)\right)\right]
$$

where $\lambda= \pm 1$ is the beam helicity. The coefficients $c_{n}$ and $s_{n}$ are parameterized by combinations of CFFs, whose exact expressions for an unpolarized target can be found in [17. In this thesis, the choice was made to take only the contributions from the constant $\mathcal{C}^{D V C S}\left(\mathcal{F}_{++} \mathcal{F}_{++}^{*} \mid \mathcal{F}_{-+} \mathcal{F}_{+-}^{*}\right)$ term. In this approximation, we have only the $c_{0}$ harmonic:

$$
c_{0}^{D V C S} \approx C_{++, *++\mid+-, *+-}(0) \mathcal{C}^{D V C S}\left(\mathcal{F}_{++} \mathcal{F}_{++}^{*} \mid \mathcal{F}_{+-} \mathcal{F}_{+-}^{*}\right)
$$

with

$$
C_{++, *+\mid+-, *+-}(0)=2 \frac{2-2 y+y^{2}+\frac{\epsilon^{2}}{2} y^{2}}{1+\epsilon^{2}}
$$

and

$$
\mathcal{C}^{D V C S}\left(\mathcal{F}_{++} \mathcal{F}_{++}^{*} \mid \mathcal{F}_{+-} \mathcal{F}_{+-}^{*}\right)=\mathcal{C}^{D V C S}\left(\mathcal{F}_{++}, \mathcal{F}_{++}^{*}\right)+\mathcal{C}^{D V C S}\left(\mathcal{F}_{+-}, \mathcal{F}_{+-}^{*}\right)
$$

where the definition of $\mathcal{C}^{D V C S}\left(\mathcal{F}_{a b}, \mathcal{F}_{c d}^{*}\right)$ is given in equation A.3.

\section{A.3 The DVCS-BH Interference term}

The interference term is given by the following expression:

$$
\mathcal{I}=\frac{ \pm e^{6}}{y^{3} x_{B} t \mathcal{P}_{1}\left(\phi_{\gamma \gamma}\right) \mathcal{P}_{2}\left(\phi_{\gamma \gamma}\right)}\left[c_{0}^{I}+\sum_{n=1}^{3}\left(c_{n}^{I} \cos \left(n \phi_{\gamma \gamma}\right)+\lambda s_{n}^{I} \sin \left(n \phi_{\gamma \gamma}\right)\right)\right]
$$

where $\lambda= \pm 1$ is the beam helicity and 


$$
\begin{gathered}
\mathcal{P}_{1}\left(\phi_{\gamma \gamma}\right)=-\frac{1}{y\left(1+\epsilon^{2}\right)}\left[J+2 K \cos \phi_{\gamma \gamma}\right] \\
\mathcal{P}_{2}\left(\phi_{\gamma \gamma}\right)=1+\frac{t}{Q^{2}}+\frac{1}{y\left(1+\epsilon^{2}\right)}\left[J+2 K \cos \phi_{\gamma \gamma}\right] \\
J=\left(1-y-\frac{y \epsilon^{2}}{2}\right)\left(t+\frac{t}{Q^{2}}\right)-\left(1-x_{B}\right)(2-y) \frac{t}{Q^{2}}
\end{gathered}
$$

The real (imaginary) part of this term is accessed experimentally from the unpolarized (polarized) cross section,

$$
\frac{1}{2}(\overrightarrow{d \sigma}+(-) \overleftarrow{d \sigma})
$$

so the helicity-dependent (independent) terms $s_{n}^{I}\left(c_{n}^{I}\right)$ contributions drop out and we are left with:

$$
\begin{gathered}
\mathcal{I}_{\text {unpol }}=\frac{e^{6}}{y^{3} x_{B} t \mathcal{P}_{1}\left(\phi_{\gamma \gamma}\right) \mathcal{P}_{2}\left(\phi_{\gamma \gamma}\right)}\left[c_{0}^{I}+\sum_{n=1}^{3} c_{n}^{I} \cos \left(n \phi_{\gamma \gamma}\right)\right] \\
\mathcal{I}_{\text {pol }}=\frac{e^{6}}{y^{3} x_{B} t \mathcal{P}_{1}\left(\phi_{\gamma \gamma}\right) \mathcal{P}_{2}\left(\phi_{\gamma \gamma}\right)}\left[\sum_{n=1}^{3} \lambda s_{n}^{I} \sin \left(n \phi_{\gamma \gamma}\right)\right]
\end{gathered}
$$

The coefficients $c_{n}$ and $s_{n}$ are parameterized by combinations of CFFs, whose exact expressions for an unpolarized target can be found in [17]. In this document, the choice was made to take only the contributions from the $\mathcal{C}^{I}\left(\mathcal{F}_{++}\right)$and $\mathcal{C}^{I}\left(\mathcal{F}_{0+}\right)$ terms. In this approximation, we have the following harmonics:

$$
\begin{gathered}
c_{0}^{I}=C_{++}(0) \operatorname{ReC}^{I}\left(\mathcal{F}_{++}\right)+C_{0+}(0) \operatorname{ReC}^{I}\left(\mathcal{F}_{0+}\right) \\
c_{1}^{I}=C_{++}(1) \operatorname{ReC}^{I}\left(\mathcal{F}_{++}\right)+C_{0+}(1) \operatorname{Re}^{I}\left(\mathcal{F}_{0+}\right) \\
c_{2}^{I}=C_{++}(2) \operatorname{Re} \mathcal{C}^{I}\left(\mathcal{F}_{++}\right)+C_{0+}(2) \operatorname{Re} \mathcal{C}^{I}\left(\mathcal{F}_{0+}\right) \\
c_{3}^{I}=C_{++}(3) \operatorname{Re} \mathcal{C}^{I}\left(\mathcal{F}_{++}\right) \\
s_{1}^{I}=S_{++}(1) \operatorname{ImC}^{I}\left(\mathcal{F}_{++}\right)+S_{0+}(1) \operatorname{Im} \mathcal{C}^{I}\left(\mathcal{F}_{0+}\right)
\end{gathered}
$$




$$
s_{2}^{I}=S_{++}(2) \operatorname{Im} \mathcal{C}^{I}\left(\mathcal{F}_{++}\right)+S_{0+}(2) \operatorname{Im} \mathcal{C}^{I}\left(\mathcal{F}_{0+}\right)
$$

and from 17

$$
s_{3}^{I}=0
$$

The terms $C_{++/ 0+}(n)$ and $S_{++/ 0+}(n)$ are defined as follows:

$$
\begin{array}{r}
C_{++}(0)=-\frac{4(2-y)\left(1+\sqrt{1+\epsilon^{2}}\right)}{\left(1+\epsilon^{2}\right)^{2}}\left\{\frac{\tilde{K}^{2}(2-y)^{2}}{Q^{2} \sqrt{1+\epsilon^{2}}}+\frac{t}{Q^{2}}\left(1-y-\frac{\epsilon^{2}}{4} y^{2}\right)\left(2-x_{B}\right)\right. \\
\left.\left(1+\frac{2 x_{B}\left(2-x_{B}+\frac{\sqrt{1+\epsilon^{2}}-1}{2}+\frac{\epsilon^{2}}{2 x_{B}}\right) \frac{t}{Q^{2}}+\epsilon^{2}}{\left(2-x_{B}\right)\left(1+\sqrt{1+\epsilon^{2}}\right)}\right)\right\} \\
C_{++}(1)=\frac{-16 K\left(1-y-\frac{\epsilon^{2}}{4} y^{2}\right)}{\left(1+\epsilon^{2}\right)^{5 / 2}}\left\{\left(1+\left(1-x_{B}\right) \frac{\sqrt{\epsilon^{2}+1}-1}{2 x_{B}}+\frac{\epsilon^{2}}{4 x_{B}}\right) \frac{x_{B} t}{Q^{2}}-\frac{3 \epsilon^{2}}{4}\right\}-4 K \\
\left(2-2 y+y^{2}+\frac{\epsilon^{2}}{2} y^{2}\right) \frac{1+\sqrt{1+\epsilon^{2}}-\epsilon^{2}}{\left(1+\epsilon^{2}\right)^{5 s / 2}} \\
\left\{1-\left(1-3 x_{B}\right) \frac{t}{Q^{2}}+\frac{1-\sqrt{1+\epsilon^{2}}+3 \epsilon^{2}}{1+\sqrt{1+\epsilon^{2}}-\epsilon^{2}} \frac{x_{B} t}{Q^{2}}\right\}
\end{array}
$$

$C_{++}(2)=\frac{8(2-y)\left(1-y-\frac{\epsilon^{2}}{4} y^{2}\right)}{\left(1+\epsilon^{2}\right)^{2}}\left\{\frac{2 \epsilon^{2}}{\sqrt{1+\epsilon^{2}}\left(1+\sqrt{1+\epsilon^{2}}\right)} \frac{\tilde{K}^{2}}{Q^{2}}+\frac{x_{B} t t^{\prime}}{Q^{4}}\left(1-x_{B}-\frac{\sqrt{1+\epsilon^{2}}-1}{2}+\frac{\epsilon^{2}}{2 x_{B}}\right)\right\}$

$C_{++}(3)=-8 K\left(1-y-\frac{\epsilon^{2}}{4} y^{2}\right) \frac{\sqrt{1+\epsilon^{2}}-1}{\left(1+\epsilon^{2}\right)^{5 / 2}}\left\{\left(1-x_{B}\right) \frac{t}{Q^{2}}+\frac{\sqrt{1+\epsilon^{2}}-1}{2}\left(1+\frac{t}{Q^{2}}\right)\right\}$

$C_{0+}(0)=\frac{12 \sqrt{2} K(2-y) \sqrt{1-y-\frac{\epsilon^{2}}{4} y^{2}}}{\left(1+\epsilon^{2}\right)^{5 / 2}}\left\{\epsilon^{2}+\frac{2-6 x_{B}-\epsilon^{2}}{3} \frac{t}{Q^{2}}\right\}$

$$
\begin{array}{r}
C_{0+}(1)=\frac{8 \sqrt{2} \sqrt{1-y-\frac{\epsilon^{2}}{4} y^{2}}}{\left(1+\epsilon^{2}\right)^{5 / 2}}\left\{(2-y)^{2} \frac{t^{\prime}}{Q^{2}}\left(1-x_{B}+\frac{\left(1-x_{B}\right) x_{B}+\frac{\epsilon^{2}}{4}}{\sqrt{1+\epsilon^{2}}} \frac{t^{\prime}}{Q^{2}}\right)+\frac{1-y-\frac{\epsilon^{2}}{4} y^{2}}{\sqrt{1+\epsilon^{2}}}\right. \\
\left.\left(1-\left(1-2 x_{B}\right) \frac{t}{Q^{2}}\right)\left(\epsilon^{2}-2\left(1+\frac{\epsilon^{2}}{2 x_{B}}\right) \frac{x_{B} t}{Q^{2}}\right)\right\}
\end{array}
$$




$$
\begin{aligned}
& C_{0+}(2)=-\frac{8 \sqrt{2} K(2-y) \sqrt{1-y-\frac{\epsilon^{2}}{4} y^{2}}}{\left(1+\epsilon^{2}\right)^{5 / 2}}\left(1+\frac{\epsilon^{2}}{2}\right)\left\{1+\frac{1+\frac{\epsilon^{2}}{2 x_{B}}}{1+\frac{\epsilon^{2}}{2}} \frac{x_{B} t}{Q^{2}}\right\} \\
& S_{++}(1)=\frac{8 K(2-y) y}{1+\epsilon^{2}}\left\{1+\frac{1-x_{B}+\frac{\sqrt{1+\epsilon^{2}}-1}{2}}{1+\epsilon^{2}} \frac{t^{\prime}}{Q^{2}}\right\} \\
& S_{++}(2)=-\frac{4\left(1-y-\frac{\epsilon^{2}}{4} y^{2}\right) y}{\left(1+\epsilon^{2}\right)^{3 / 2}}\left(1+\sqrt{1+\epsilon^{2}}-2 x_{B}\right) \frac{t^{\prime}}{Q^{2}}\left\{\frac{\epsilon^{2}-x_{B}\left(\sqrt{1+\epsilon^{2}}-1\right)}{1+\sqrt{1+\epsilon^{2}}-2 x_{B}}-\frac{2 x_{B}+\epsilon^{2}}{2 \sqrt{1+\epsilon^{2}}} \frac{t^{\prime}}{Q^{2}}\right\} \\
& S_{0+}(1)=\frac{8 \sqrt{2}(2-y) y \sqrt{1-y-\frac{\epsilon^{2}}{4} y^{2}}}{\left(1+\epsilon^{2}\right)^{2}} \frac{t \tilde{K}^{2}}{Q^{4}} \\
& S_{0+}(2)=\frac{8 \sqrt{2} K y \sqrt{1-y-\frac{\epsilon^{2}}{4} y^{2}}}{\left(1+\epsilon^{2}\right)^{2}}\left(1+\frac{\epsilon^{2}}{2}\right)\left\{1+\frac{1+\frac{\epsilon^{2}}{2 x_{B}}}{1+\frac{\epsilon^{2}}{2}} \frac{x_{B} t}{Q^{2}}\right\}
\end{aligned}
$$


Appendix B

Unpolarized and helicity-dependent DVCS cross sections

B.1 Unpolarized Cross Sections 

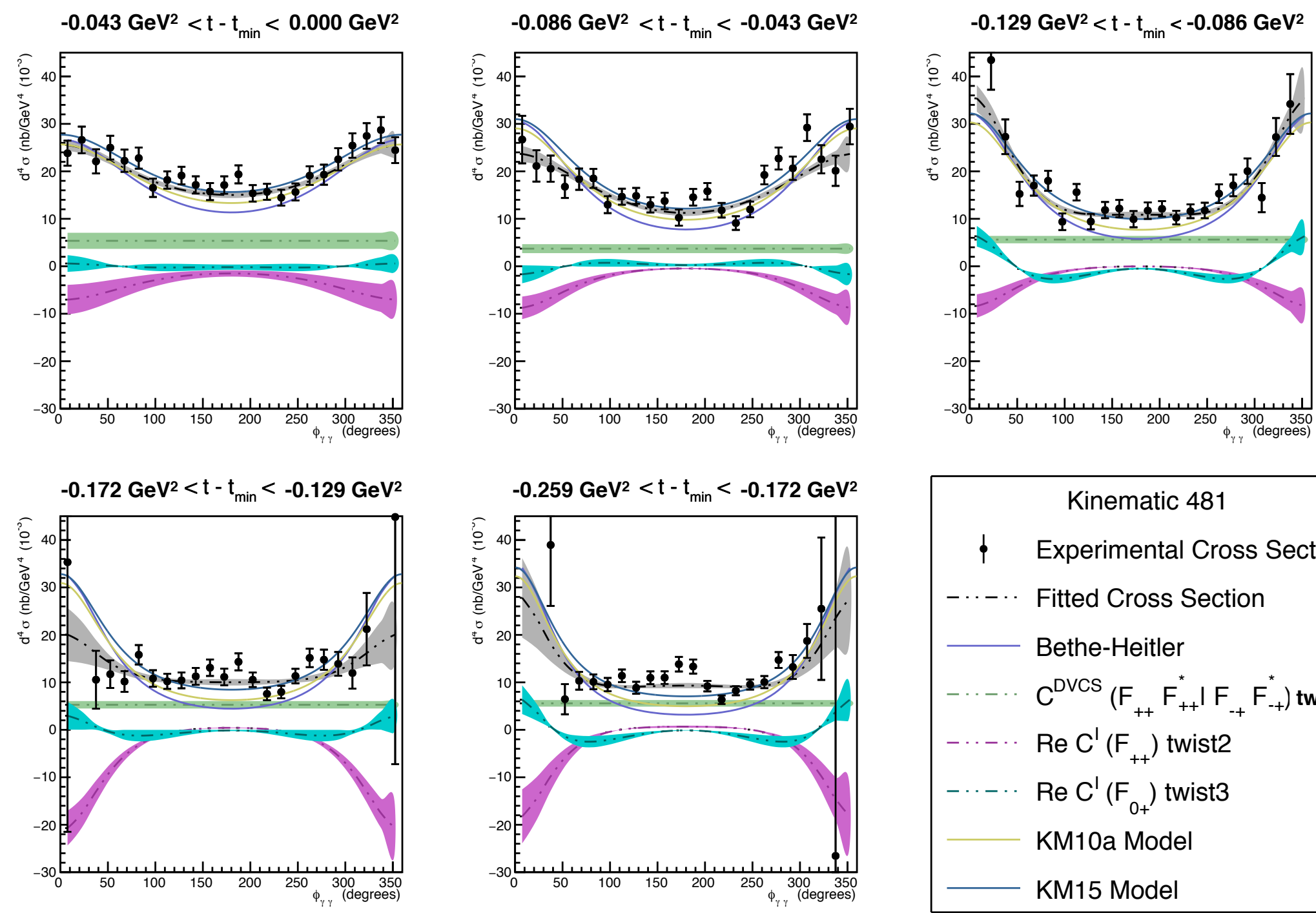

\begin{tabular}{|cl|}
\hline \multicolumn{1}{|c|}{ Kinematic 481} \\
$\cdots \cdots$ & Experimental Cross Section \\
$-\cdots$ & Fitted Cross Section \\
& Bethe-Heitler \\
$-\cdots-\cdots$ & $C^{\text {DVCS }}\left(F_{++} F_{++}^{*} F_{-+} F_{++}^{*}\right)$ twist2 \\
$-\cdots-\cdots$ & $\operatorname{Re~} C^{l}\left(F_{++}\right)$twist2 \\
$-\cdots-\cdots$ & $\operatorname{Re~} C^{l}\left(F_{0+}\right)$ twist3 \\
& KM10a Model \\
& KM15 Model \\
\hline
\end{tabular}

Figure B.1: Unpolarized cross sections for kinematic setting $481\left(\chi^{2} /\right.$ dof $\left.=2.03\right)$. Error bars are statistical only. The uncertainty introduced from the choice of missing mass cut is quoted for each bin in the tables of cross sections (Appendix $\mathrm{D}$ ). 

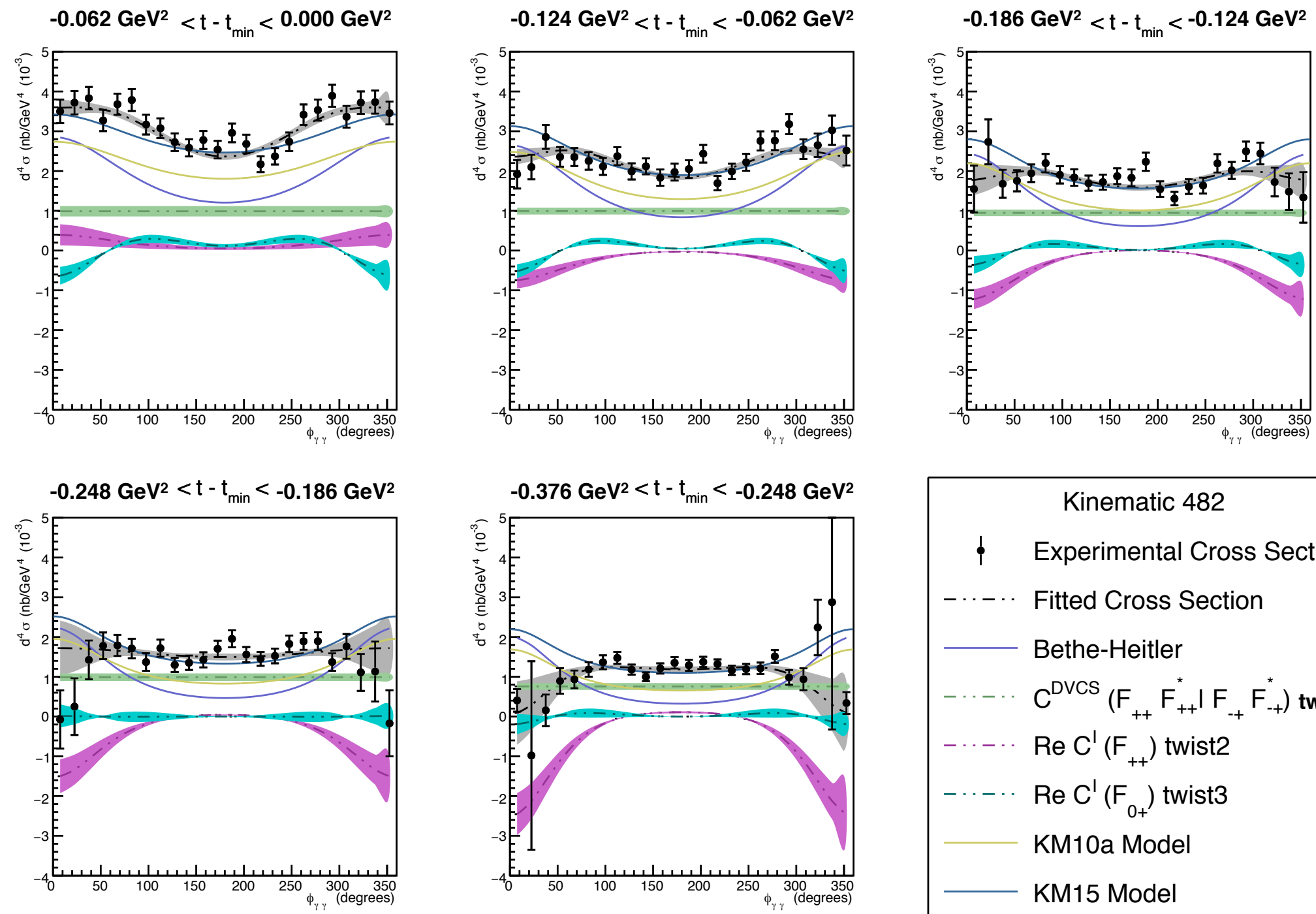

\begin{tabular}{|c|c|}
\hline & Kinematic 482 \\
\hline$\phi$ & Experimental Cross Section \\
\hline$-\cdots-\cdots$ & Fitted Cross Section \\
\hline & Bethe-Heitler \\
\hline$-\cdots-$ & $\mathrm{C}^{\text {DVCS }}\left(\mathrm{F}_{++} \mathrm{F}_{++}^{*} \mid \mathrm{F}_{-+} \mathrm{F}_{-+}^{*}\right)$ twist? \\
\hline$-\cdots-$ & $\operatorname{Re} C^{\prime}\left(F_{++}\right)$twist2 \\
\hline$-\cdots-\cdots$ & $\operatorname{Re} C^{\prime}\left(F_{0+}\right)$ twist3 \\
\hline & KM10a Model \\
\hline & KM15 Model \\
\hline
\end{tabular}

Figure B.2: Unpolarized cross sections for kinematic setting $482\left(\chi^{2} /\right.$ dof $\left.=1.35\right)$. Error bars are statistical only. The uncertainty introduced from the choice of missing mass cut is quoted for each bin in the tables of cross sections (Appendix D). 

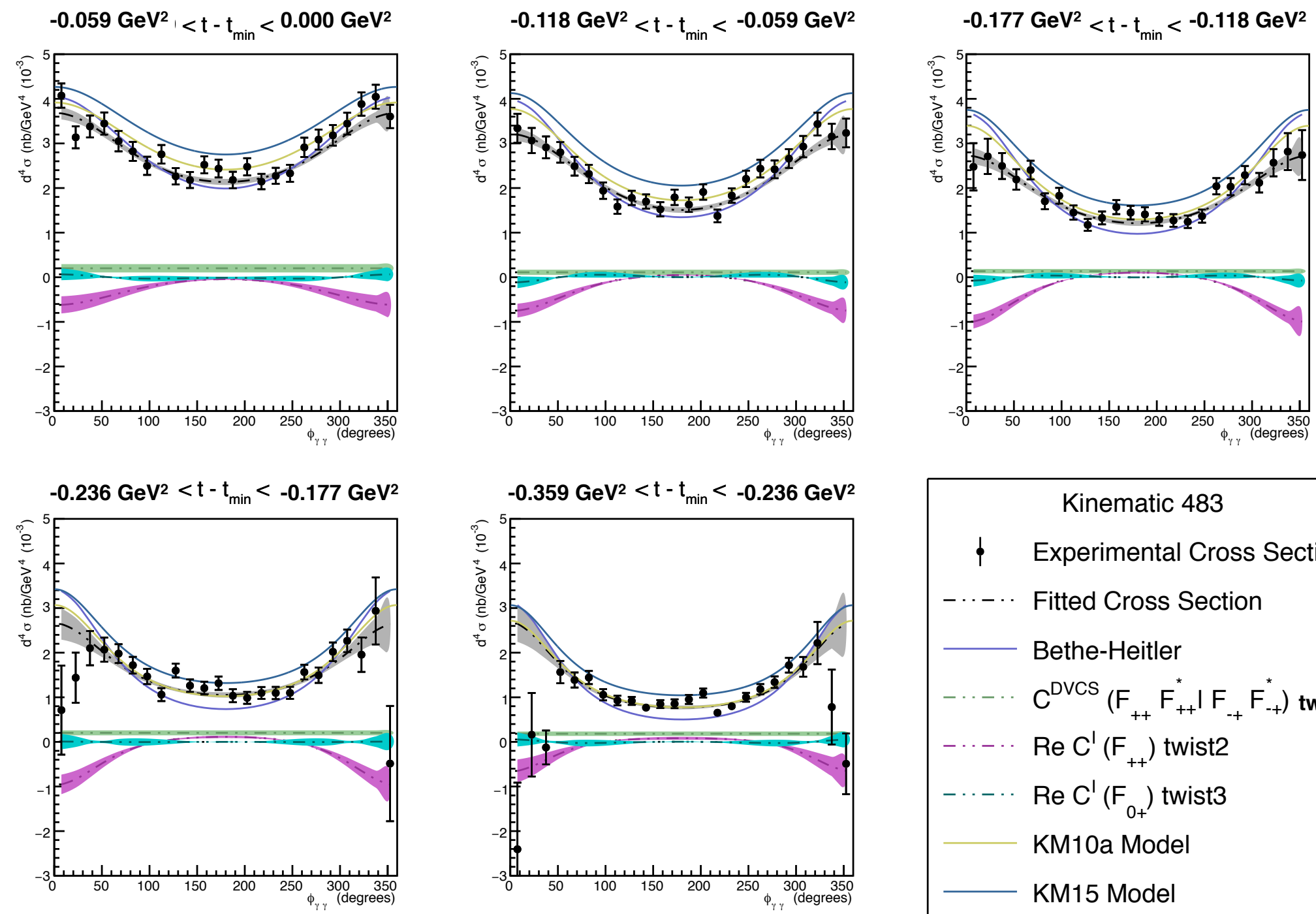

\begin{tabular}{|c|c|}
\hline & Kinematic 483 \\
\hline$\phi$ & Experimental Cross Section \\
\hline$-\cdots-$ & Fitted Cross Section \\
\hline & Bethe-Heitler \\
\hline$-\cdots-$ & $C^{\text {DVCS }}\left(F_{++} F_{++}^{*} \mid F_{-+} F_{-+}^{*}\right)$ twist? \\
\hline$-\cdots-$ & $\operatorname{Re} C^{\prime}\left(F_{++}\right)$twist2 \\
\hline$-\cdots-$ & $\operatorname{Re} C^{\prime}\left(F_{0+}\right)$ twist3 \\
\hline & KM10a Model \\
\hline 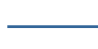 & KM15 Model \\
\hline
\end{tabular}

Figure B.3: Unpolarized cross sections for kinematic setting $483\left(\chi^{2} /\right.$ dof $\left.=1.68\right)$. Error bars are statistical only. The uncertainty introduced from the choice of missing mass cut is quoted for each bin in the tables of cross sections (Appendix D). 

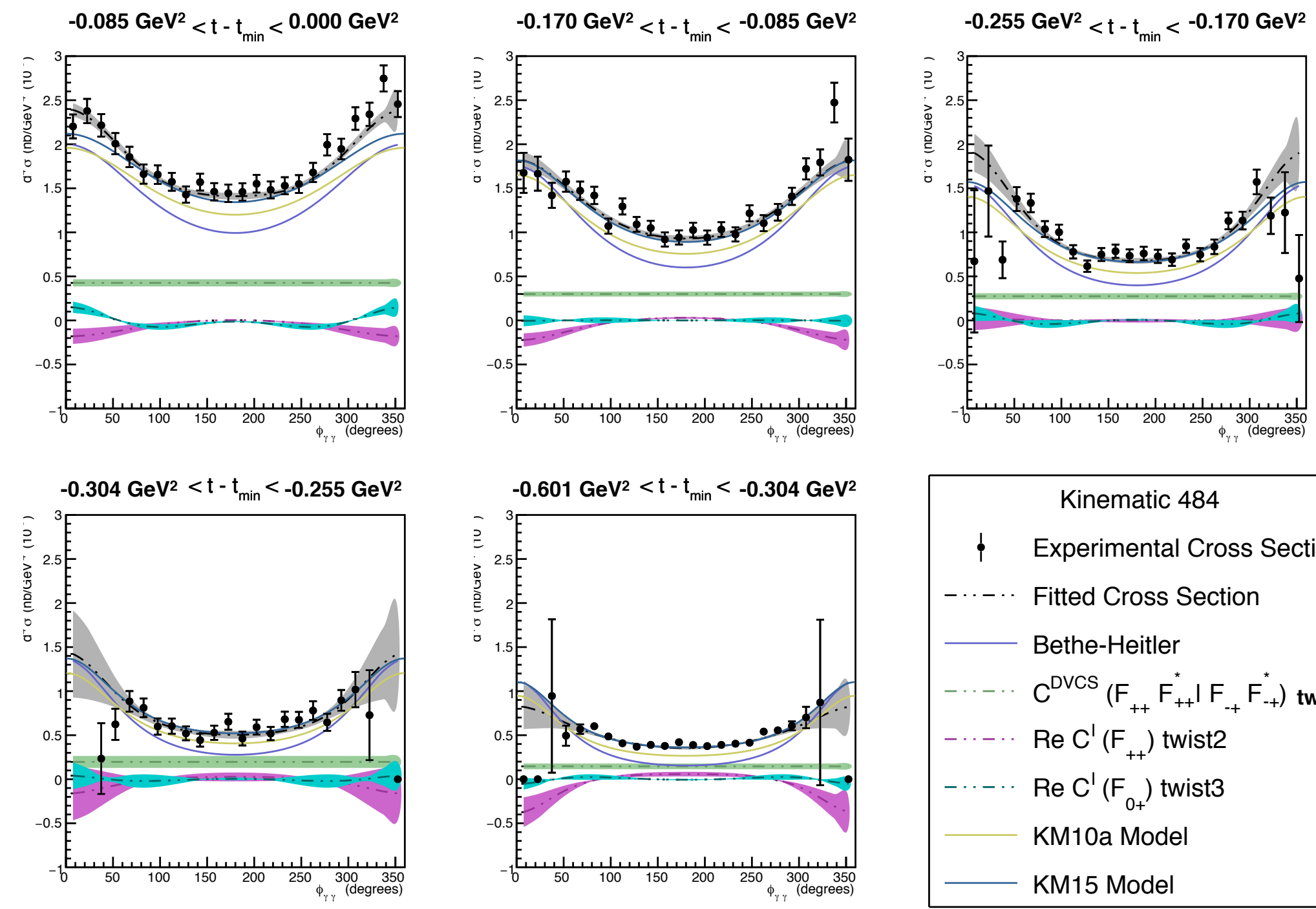

\begin{tabular}{|c|c|}
\hline & Kinematic 484 \\
\hline$\phi$ & Experimental Cross Section \\
\hline$-\cdots-$ & Fitted Cross Section \\
\hline & Bethe-Heitler \\
\hline & $C^{\text {DVCS }}\left(F_{++} F_{++}^{*} \mid F_{-+} F_{-+}^{*}\right)$ twist \\
\hline$-\cdots-$ & $\operatorname{Re} C^{\prime}\left(F_{++}\right)$twist2 \\
\hline$-\cdots-$ & $\operatorname{Re} C^{\prime}\left(F_{0+}\right)$ twist3 \\
\hline & KM10a Model \\
\hline & KM15 Model \\
\hline
\end{tabular}

Figure B.4: Unpolarized cross sections for kinematic setting $484\left(\chi^{2} / d o f=1.39\right)$. Error bars are statistical only. The uncertainty introduced from the choice of missing mass cut is quoted for each bin in the tables of cross sections (Appendix $\mathrm{D}$ ). 


\section{B.2 Helicity-Dependent Cross Sections}
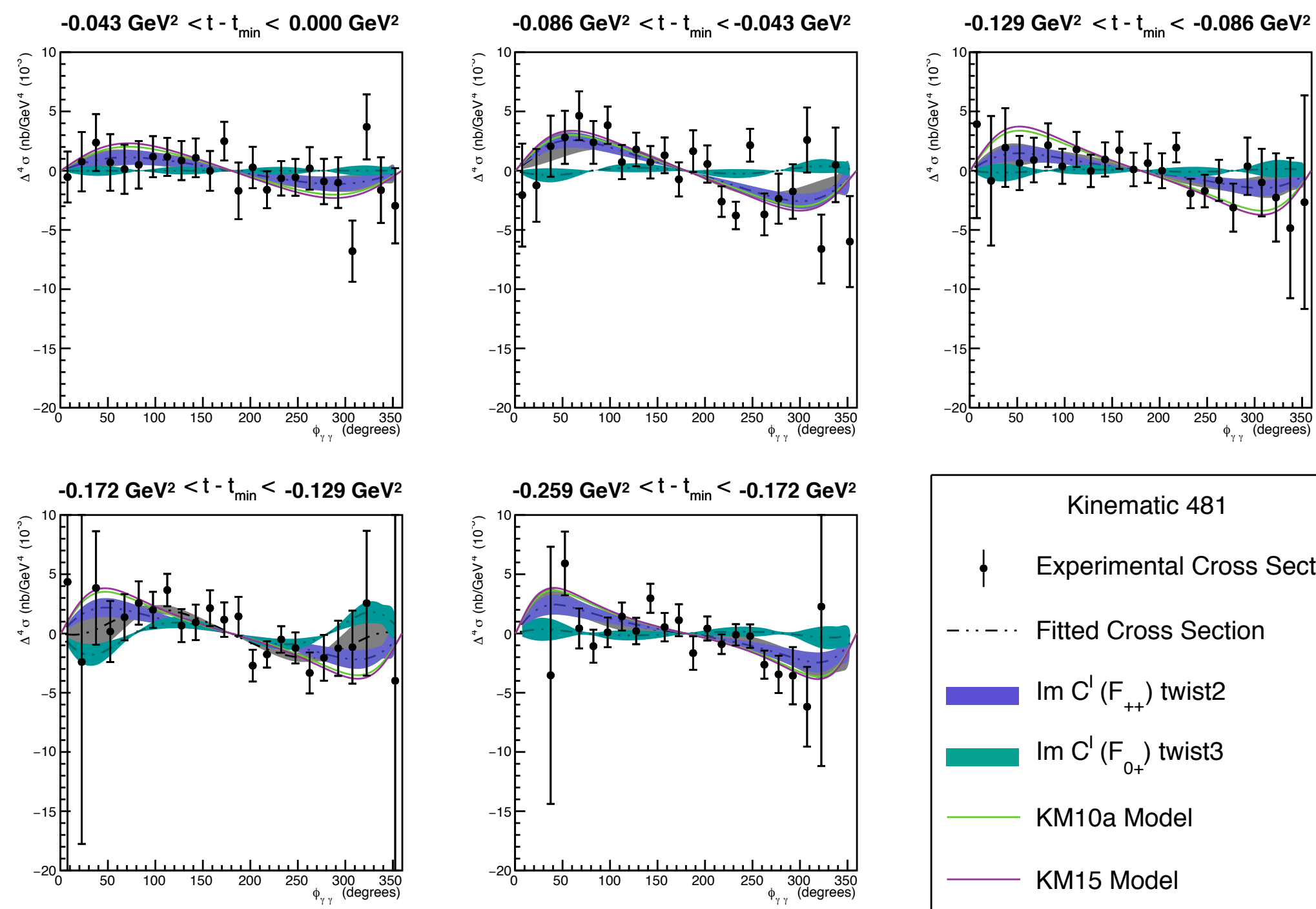

\begin{tabular}{|cc|}
\hline & Kinematic 481 \\
$-\cdots \cdots$ & Experimental Cross Section \\
& Im $C^{\prime}\left(F_{++}\right)$twist2 \\
& Im C ${ }^{\prime}\left(F_{0+}\right)$ twist3 \\
& $\mathrm{KM} 10 \mathrm{a}$ Model \\
$\mathrm{KM} 15$ Model
\end{tabular}

Figure B.5: Helicity-dependent cross sections for kinematic setting $481\left(\chi^{2} /\right.$ dof $\left.=0.78\right)$. Error bars are statistical only. The uncertainty introduced from the choice of missing mass cut is quoted for each bin in the tables of cross sections (Appendix E). 

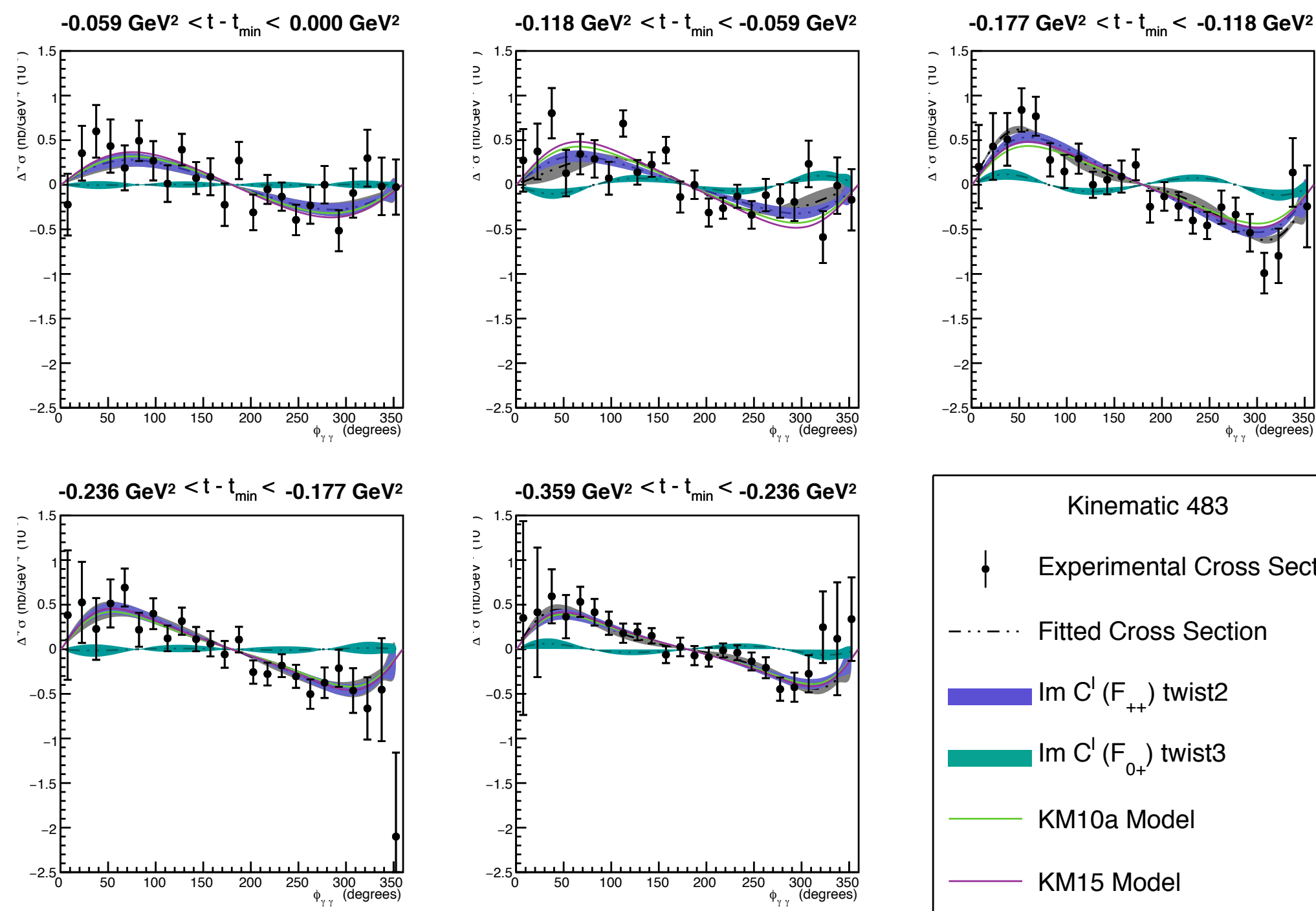

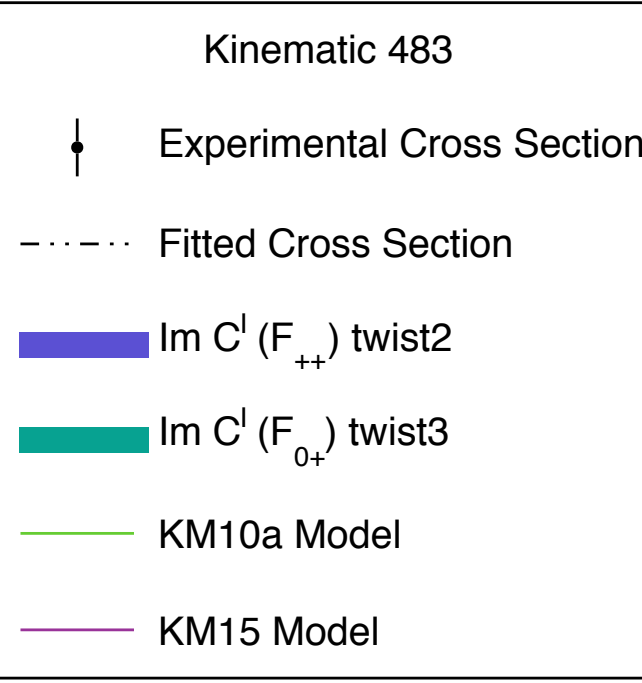

Figure B.7: Helicity-dependent cross sections for kinematic setting $483\left(\chi^{2} /\right.$ dof $\left.=0.95\right)$. Error bars are statistical only. The uncertainty introduced from the choice of missing mass cut is quoted for each bin in the tables of cross sections (Appendix E). 
Appendix C

Fitted Number of Events 

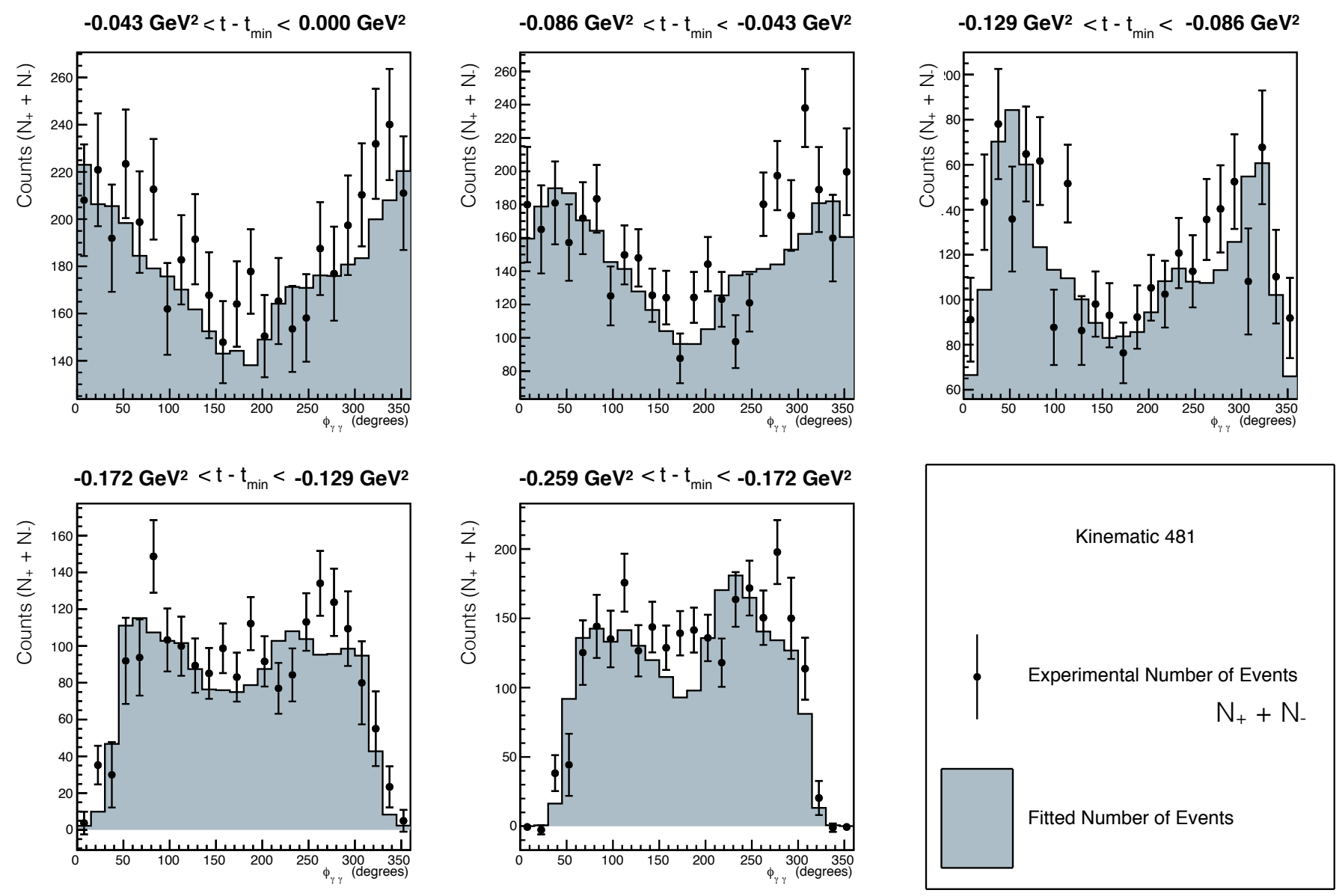

Figure C.1: Unpolarized number of events for kinematic setting 481. The black circles represent the experimental number of events, and the grey histograms represent the fitted number of simulated events $\left(\chi^{2} /\right.$ dof $\left.=2.03\right)$. 

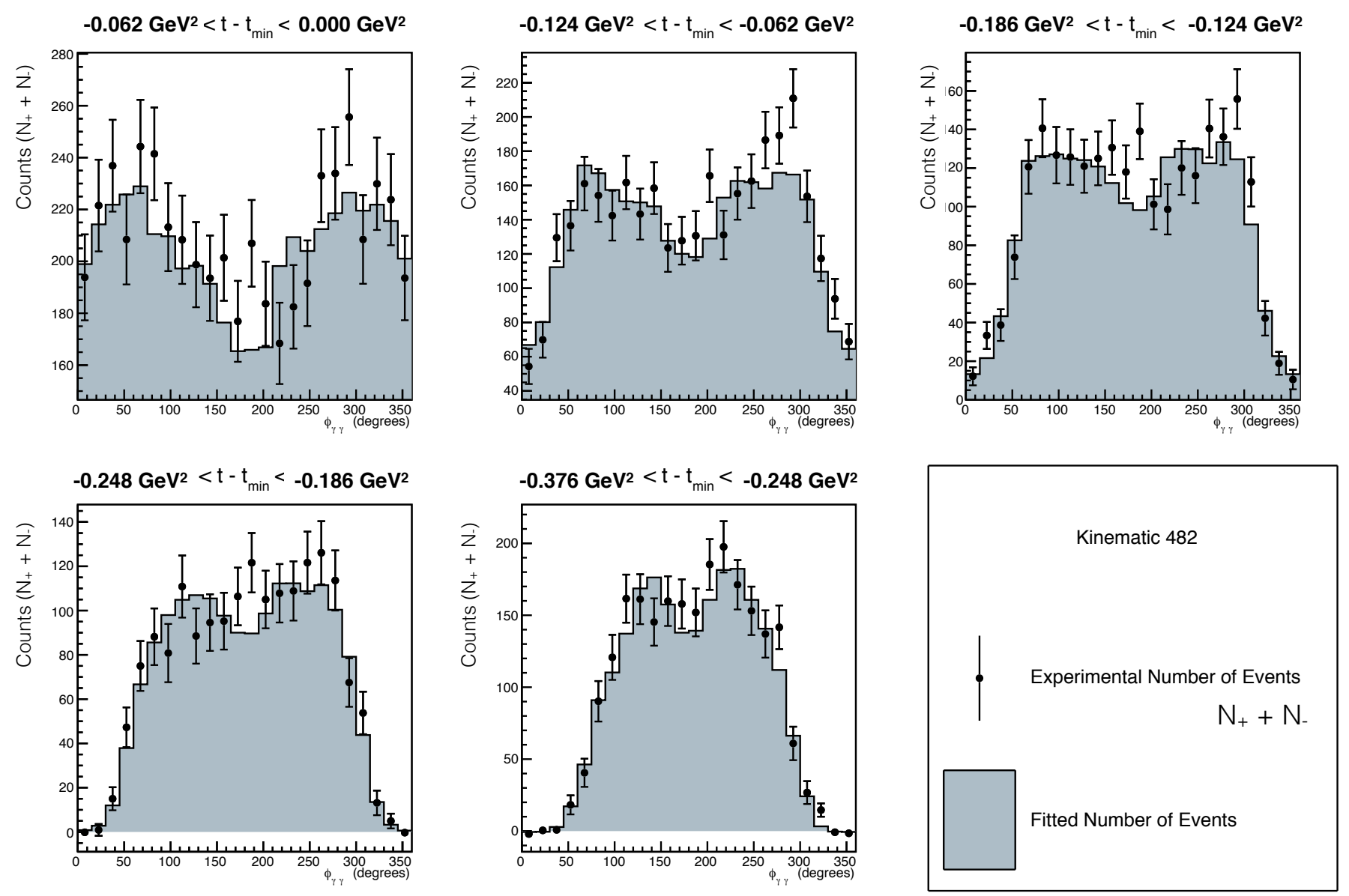

Figure C.2: Unpolarized number of events for kinematic setting 482. The black circles represent the experimental number of events, and the grey histograms represent the fitted number of simulated events $\left(\chi^{2} /\right.$ dof $\left.=1.35\right)$. 

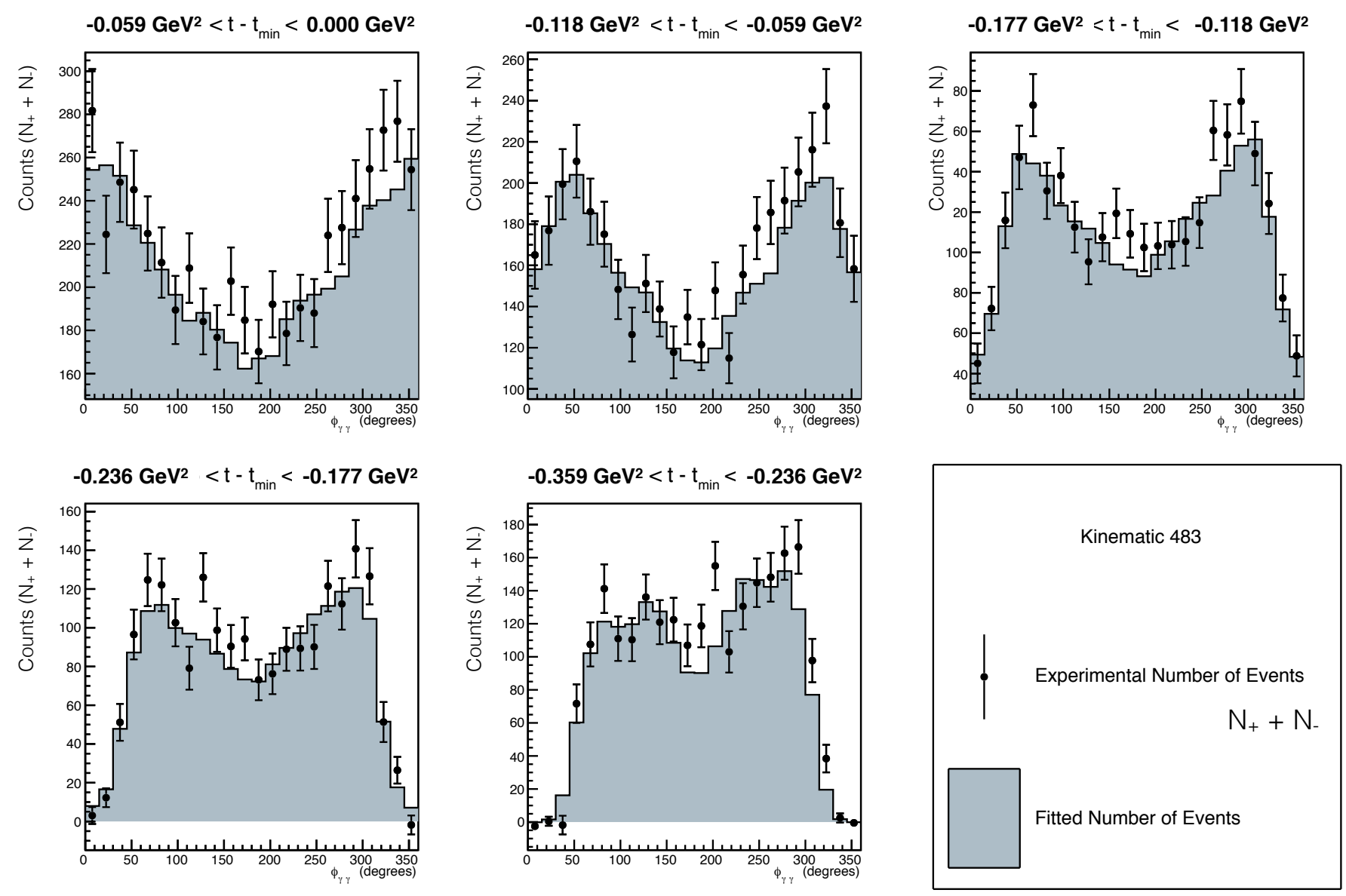

Figure C.3: Unpolarized number of events for kinematic setting 483. The black circles represent the experimental number of events, and the grey histograms represent the fitted number of simulated events $\left(\chi^{2} / d o f=1.68\right)$. 

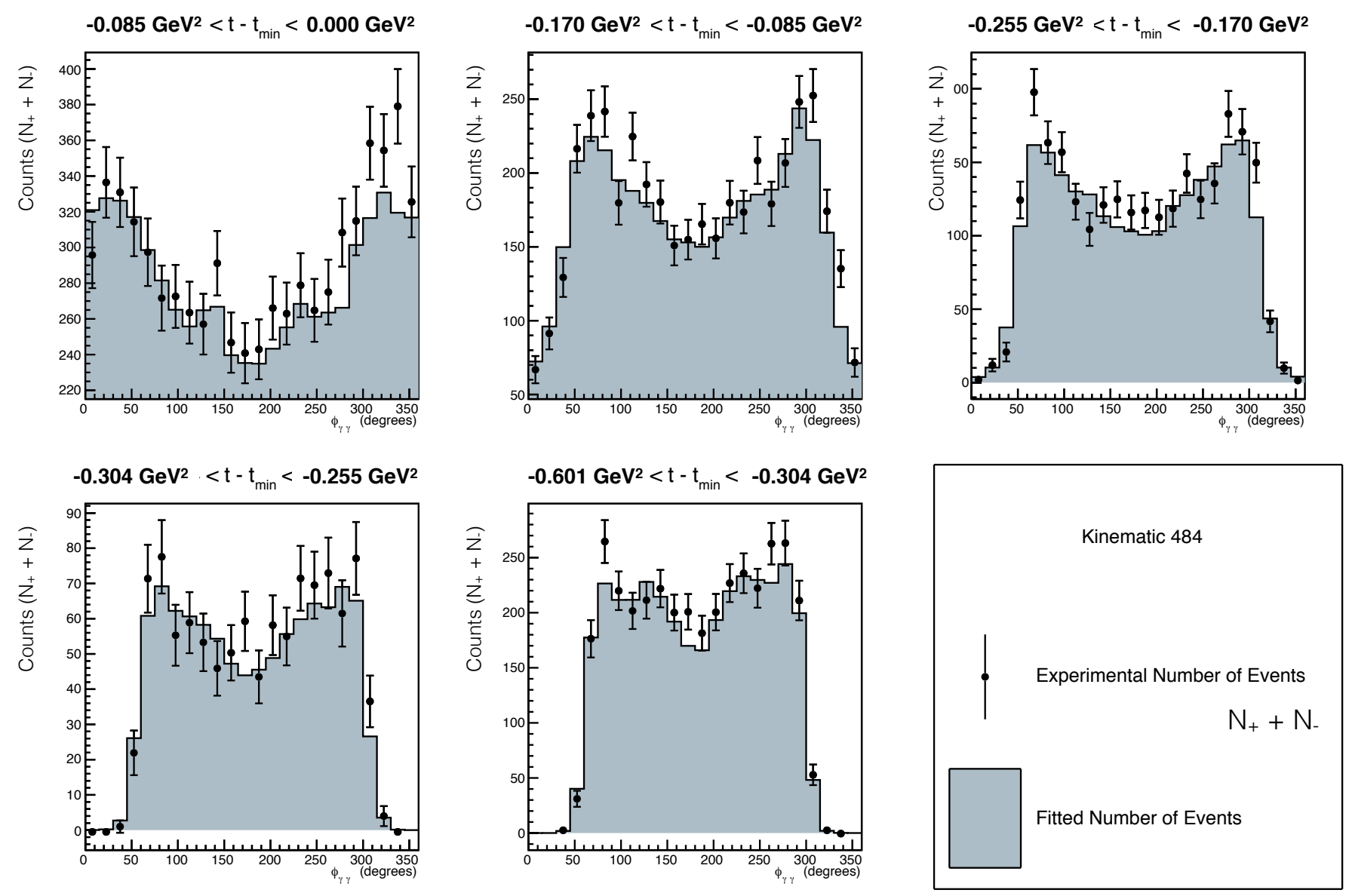

Figure C.4: Unpolarized number of events for kinematic setting 484. The black circles represent the experimental number of events, and the grey histograms represent the fitted number of simulated events $\left(\chi^{2} / d o f=1.39\right)$. 

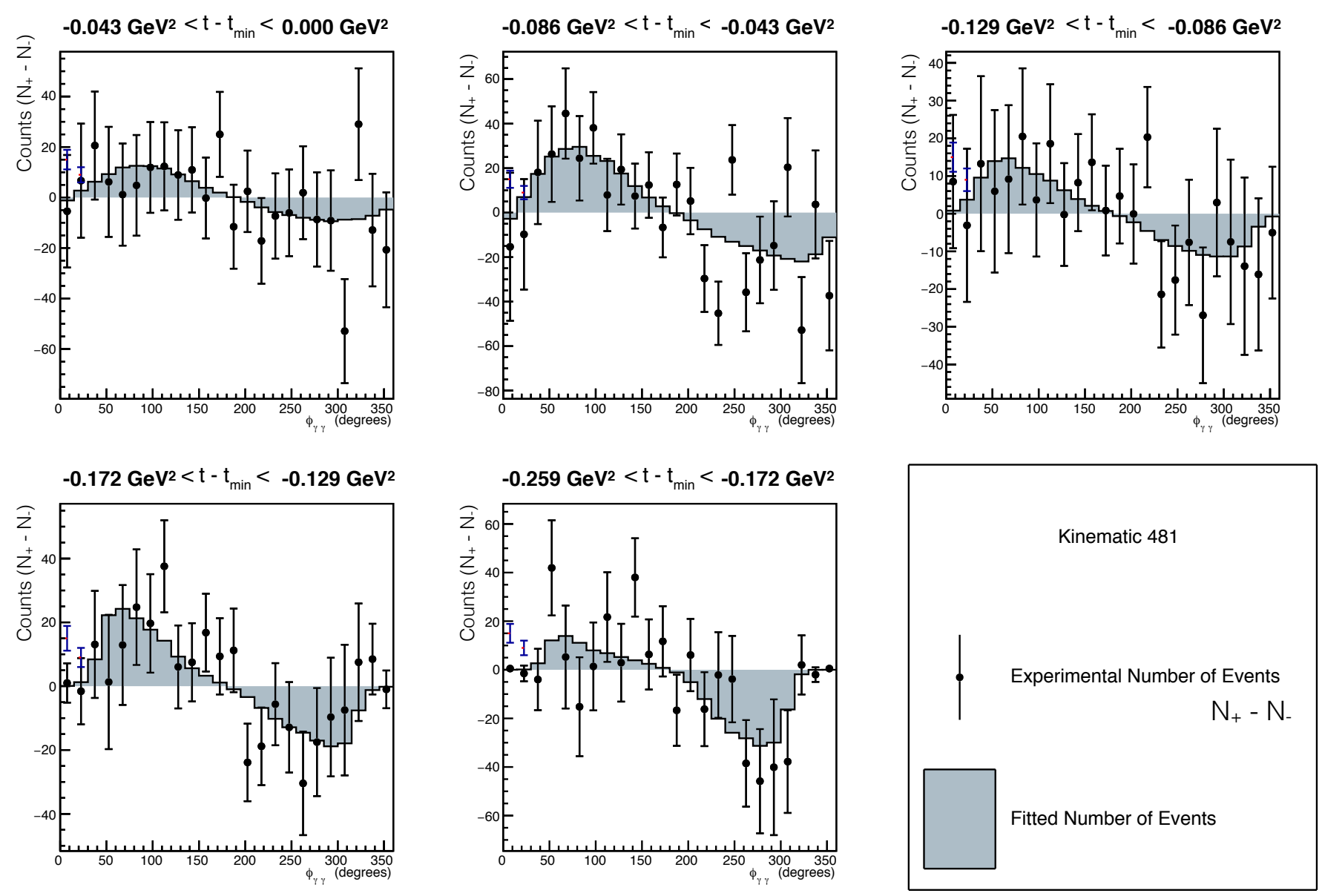

Figure C.5: Helicity-dependent number of events for kinematic setting 481. The black circles represent the experimental number of events, and the grey histograms represent the fitted number of simulated events $\left(\chi^{2} /\right.$ dof $\left.=0.78\right)$. 

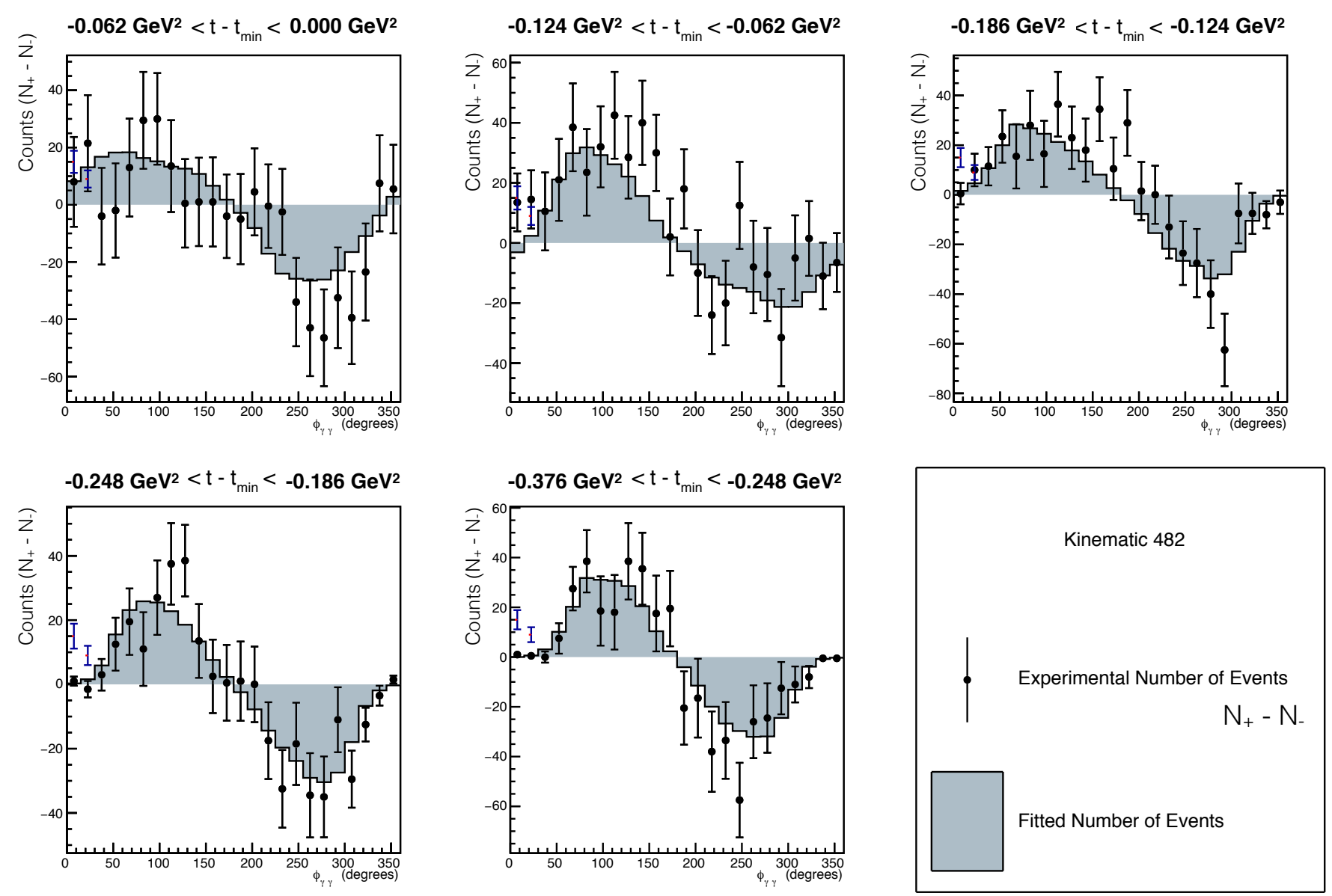

Figure C.6: Helicity-dependent number of events for kinematic setting 482 . The black circles represent the experimental number of events, and the grey histograms represent the fitted number of simulated events $\left(\chi^{2} /\right.$ dof $\left.=0.94\right)$. 

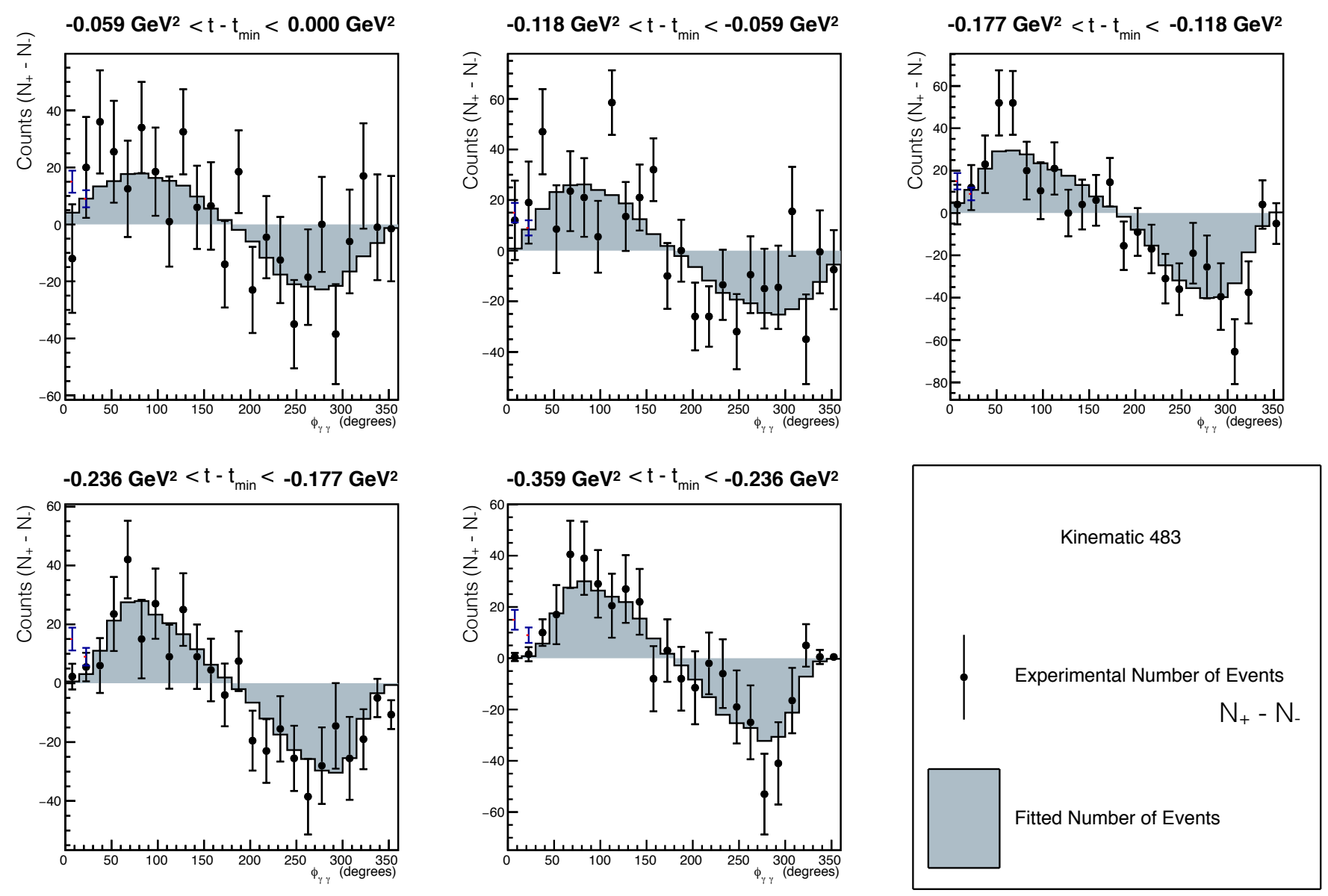

Figure C.7: Helicity-dependent number of events for kinematic setting 483. The black circles represent the experimental number of events, and the grey histograms represent the fitted number of simulated events $\left(\chi^{2} /\right.$ dof $\left.=0.95\right)$. 

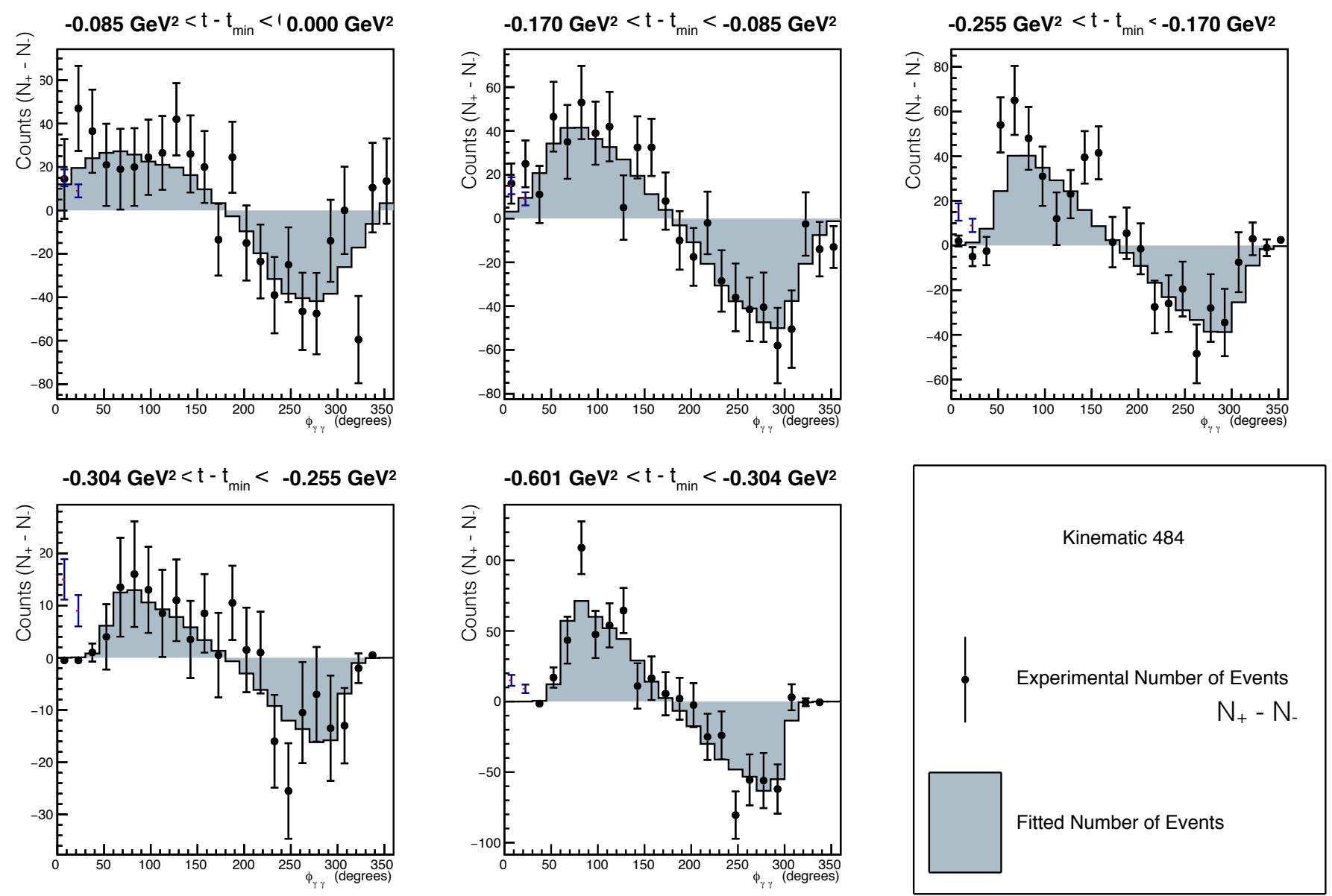

Figure C.8: Helicity-dependent number of events for kinematic setting 484 . The black circles represent the experimental number of events, and the grey histograms represent the fitted number of simulated events $\left(\chi^{2} /\right.$ dof $\left.=1.02\right)$. 
Appendix D

\title{
Tables of unpolarized DVCS cross sections
}

\author{
D.1 Unpolarized Cross Sections
}




\begin{tabular}{|c|c|c|c|c|c|c|c|c|c|c|}
\hline $\begin{array}{l}\phi_{\gamma \gamma} \\
(\mathrm{deg})\end{array}$ & \multicolumn{2}{|c|}{$\begin{array}{c}\left\langle x_{B}\right\rangle=0.483 \\
\left\langle Q^{2}\right\rangle=2.707 \mathrm{GeV}^{2} \\
\left\langle t^{\prime}\right\rangle=-0.022 \mathrm{GeV}^{2}\end{array}$} & \multicolumn{2}{|c|}{$\begin{array}{c}\left\langle x_{B}\right\rangle=0.483 \\
\left\langle Q^{2}\right\rangle=2.708 \mathrm{GeV}^{2} \\
\left\langle t^{\prime}\right\rangle=-0.064 \mathrm{GeV}^{2}\end{array}$} & \multicolumn{2}{|c|}{$\begin{array}{c}\left\langle x_{B}\right\rangle=0.484 \\
\left\langle Q^{2}\right\rangle=2.713 \mathrm{GeV}^{2} \\
\left\langle t^{\prime}\right\rangle=-0.107 \mathrm{GeV}^{2}\end{array}$} & \multicolumn{2}{|c|}{$\begin{array}{c}\left\langle x_{B}\right\rangle=0.485 \\
\left\langle Q^{2}\right\rangle=2.715 \mathrm{GeV}^{2} \\
\left\langle t^{\prime}\right\rangle=-0.150 \mathrm{GeV}^{2}\end{array}$} & \multicolumn{2}{|c|}{$\begin{array}{c}\left\langle x_{B}\right\rangle=0.485 \\
\left\langle Q^{2}\right\rangle=2.717 \mathrm{GeV}^{2} \\
\left\langle t^{\prime}\right\rangle=-0.210 \mathrm{GeV}^{2}\end{array}$} \\
\hline 7.5 & $27.99 \pm 3.07$ & $\begin{array}{c}+0.89 \\
-0.31\end{array}$ & $-51.87 \pm 4.88$ & $\begin{array}{c}+1.42 \\
-3.94\end{array}$ & $45.72 \pm 9.30$ & $\begin{array}{c}+0.26 \\
-2.24\end{array}$ & $15.37 \pm 3.30$ & $\begin{array}{c}+10.54 \\
-5.11\end{array}$ & $-30.46 \pm 41.96$ & $\begin{array}{c}+14.93 \\
-0.00\end{array}$ \\
\hline 22.5 & $30.21 \pm 3.19$ & $\begin{array}{c}+0.55 \\
-0.24\end{array}$ & $21.01 \pm 3.27$ & $\begin{array}{c}+0.00 \\
-0.73\end{array}$ & $42.71 \pm 6.09$ & $\begin{array}{c}+1.16 \\
-0.00\end{array}$ & $57.17 \pm 1.66$ & $\begin{array}{l}+2.05 \\
-0.04\end{array}$ & $-48.88 \pm 59.41$ & $\begin{array}{c}+16.10 \\
-0.00\end{array}$ \\
\hline 37.5 & $26.86 \pm 3.01$ & $\begin{array}{c}+0.47 \\
-0.22\end{array}$ & $21.26 \pm 2.78$ & $\begin{array}{c}+0.41 \\
-0.52\end{array}$ & $27.31 \pm 3.65$ & $\begin{array}{l}+0.17 \\
-0.96\end{array}$ & $11.97 \pm 0.60$ & $\begin{array}{l}+0.36 \\
-0.50\end{array}$ & $39.19 \pm 13.92$ & $\begin{array}{l}+6.52 \\
-4.55\end{array}$ \\
\hline 52.5 & $28.34 \pm 2.84$ & $\begin{array}{c}+0.05 \\
-0.64\end{array}$ & $17.48 \pm 2.44$ & $\begin{array}{l}+0.25 \\
-0.29 \\
\end{array}$ & $15.58 \pm 2.56$ & $\begin{array}{l}+1.21 \\
-0.00\end{array}$ & $11.29 \pm 2.91$ & $\begin{array}{l}+0.00 \\
-1.42\end{array}$ & $6.70 \pm 3.14$ & $\begin{array}{l}+1.14 \\
-0.32\end{array}$ \\
\hline 67.5 & $25.68 \pm 2.75$ & $\begin{array}{c}+1.08 \\
-0.40 \\
\end{array}$ & $19.22 \pm 2.38$ & $\begin{array}{c}+0.81 \\
-0.79 \\
\end{array}$ & $17.74 \pm 2.14$ & $\begin{array}{c}+0.08 \\
-0.29\end{array}$ & $11.61 \pm 2.26$ & $\begin{array}{c}+1.01 \\
-0.19\end{array}$ & $10.50 \pm 1.87$ & $\begin{array}{l}+0.00 \\
-0.41\end{array}$ \\
\hline 82.5 & $27.78 \pm 2.67$ & $\begin{array}{l}+1.05 \\
-0.00\end{array}$ & $20.55 \pm 2.18$ & $\begin{array}{l}+0.69 \\
-0.32\end{array}$ & $17.36 \pm 2.06$ & $\begin{array}{l}+0.23 \\
-0.66\end{array}$ & $16.80 \pm 2.13$ & $\begin{array}{l}+0.68 \\
-0.00\end{array}$ & $10.23 \pm 1.55$ & $\begin{array}{l}+0.05 \\
-0.51\end{array}$ \\
\hline 97.5 & $19.81 \pm 2.27$ & $\begin{array}{l}+0.74 \\
-0.00 \\
\end{array}$ & $14.46 \pm 1.94$ & $\begin{array}{l}+0.42 \\
-0.41 \\
\end{array}$ & $9.71 \pm 1.78$ & $\begin{array}{c}+0.31 \\
-0.01 \\
\end{array}$ & $11.96 \pm 1.85$ & $\begin{array}{l}+0.33 \\
-0.71 \\
\end{array}$ & $10.07 \pm 1.46$ & $\begin{array}{l}+0.07 \\
-0.39 \\
\end{array}$ \\
\hline 112.5 & $21.43 \pm 2.18$ & $\begin{array}{c}+0.45 \\
-0.14\end{array}$ & $16.48 \pm 1.88$ & $\begin{array}{l}+0.75 \\
-0.27\end{array}$ & $15.87 \pm 1.78$ & $\begin{array}{c}+0.49 \\
-0.48\end{array}$ & $11.28 \pm 1.73$ & $\begin{array}{l}+1.10 \\
-0.00\end{array}$ & $12.18 \pm 1.39$ & $\begin{array}{l}+0.10 \\
-0.36\end{array}$ \\
\hline 127.5 & $23.24 \pm 2.24$ & $\begin{array}{c}+0.81 \\
-0.00\end{array}$ & $15.96 \pm 1.80$ & $\begin{array}{l}+0.93 \\
-0.02\end{array}$ & $9.89 \pm 1.71$ & $\begin{array}{l}+0.11 \\
-0.58\end{array}$ & $11.44 \pm 1.84$ & $\begin{array}{l}+0.39 \\
-0.19\end{array}$ & $9.78 \pm 1.40$ & $\begin{array}{l}+0.65 \\
-0.72\end{array}$ \\
\hline 142.5 & $19.86 \pm 2.13$ & $\begin{array}{c}+0.00 \\
-0.10\end{array}$ & $13.12 \pm 1.66$ & $\begin{array}{l}+0.15 \\
-0.81\end{array}$ & $12.66 \pm 1.84$ & $\begin{array}{c}+0.64 \\
-0.20\end{array}$ & $12.54 \pm 2.00$ & $\begin{array}{l}+0.00 \\
-0.71\end{array}$ & $12.32 \pm 1.54$ & $\begin{array}{c}+0.00 \\
-0.39 \\
\end{array}$ \\
\hline 157.5 & $16.93 \pm 1.94$ & $\begin{array}{l}+0.00 \\
-0.62 \\
\end{array}$ & $14.22 \pm 1.77$ & $\begin{array}{c}+0.25 \\
-0.30\end{array}$ & $12.56 \pm 1.87$ & $\begin{array}{c}+0.26 \\
-0.38 \\
\end{array}$ & $13.59 \pm 1.82$ & $\begin{array}{c}+0.10 \\
-0.28 \\
\end{array}$ & $11.62 \pm 1.39$ & $\begin{array}{l}+0.16 \\
-0.11 \\
\end{array}$ \\
\hline 172.5 & $18.38 \pm 1.95$ & $\begin{array}{l}+0.64 \\
-0.17\end{array}$ & $9.53 \pm 1.64$ & $\begin{array}{c}+0.09 \\
-0.42 \\
\end{array}$ & $9.51 \pm 1.66$ & $\begin{array}{c}+0.27 \\
-0.32 \\
\end{array}$ & $10.91 \pm 1.73$ & $\begin{array}{l}+0.60 \\
-0.62\end{array}$ & $13.34 \pm 1.53$ & $\begin{array}{c}+0.00 \\
-0.59\end{array}$ \\
\hline 187.5 & $20.67 \pm 2.03$ & $\begin{array}{r}+0.43 \\
-0.43 \\
\end{array}$ & $14.26 \pm 1.67$ & $\begin{array}{c}+0.61 \\
-0.00 \\
\end{array}$ & $11.97 \pm 1.72$ & $\begin{array}{c}+0.57 \\
-0.00\end{array}$ & $14.49 \pm 1.82$ & $\begin{array}{c}+1.93 \\
-0.20 \\
\end{array}$ & $13.08 \pm 1.47$ & $\begin{array}{c}+0.58 \\
-0.25 \\
\end{array}$ \\
\hline 202.5 & $17.39 \pm 1.93$ & $\begin{array}{l}+0.36 \\
-0.46 \\
\end{array}$ & $16.59 \pm 1.77$ & $\begin{array}{c}+0.31 \\
-0.37 \\
\end{array}$ & $12.97 \pm 1.73$ & $\begin{array}{c}+0.05 \\
-0.15 \\
\end{array}$ & $10.90 \pm 1.58$ & $\begin{array}{c}+1.07 \\
-0.51 \\
\end{array}$ & $9.76 \pm 1.15$ & $\begin{array}{c}+0.00 \\
-0.30\end{array}$ \\
\hline 217.5 & $19.79 \pm 2.10$ & $\begin{array}{c}+0.61 \\
-0.15\end{array}$ & $13.10 \pm 1.72$ & $\begin{array}{c}+0.31 \\
-0.23\end{array}$ & $11.90 \pm 1.64$ & $\begin{array}{c}+0.00 \\
-0.70\end{array}$ & $8.58 \pm 1.52$ & $\begin{array}{c}+0.08 \\
-0.25\end{array}$ & $7.23 \pm 1.01$ & $\begin{array}{c}+0.25 \\
-0.00\end{array}$ \\
\hline 232.5 & $18.53 \pm 2.11$ & $\begin{array}{c}+0.30 \\
-0.24 \\
\end{array}$ & $10.72 \pm 1.69$ & $\begin{array}{c}+0.09 \\
-0.24\end{array}$ & $13.84 \pm 1.73$ & $\begin{array}{c}+0.33 \\
-0.16 \\
\end{array}$ & $9.53 \pm 1.58$ & $\begin{array}{c}+0.23 \\
-0.50\end{array}$ & $9.60 \pm 1.13$ & $\begin{array}{c}+0.74 \\
-0.69 \\
\end{array}$ \\
\hline 247.5 & $20.10 \pm 2.23$ & $\begin{array}{l}+0.25 \\
-0.27\end{array}$ & $14.37 \pm 1.96$ & $\begin{array}{c}+0.18 \\
-0.73\end{array}$ & $12.61 \pm 1.80$ & $\begin{array}{c}+0.21 \\
-0.76\end{array}$ & $12.80 \pm 1.77$ & $\begin{array}{c}+0.18 \\
-0.68\end{array}$ & $10.28 \pm 1.19$ & $\begin{array}{c}+0.00 \\
-0.54\end{array}$ \\
\hline 262.5 & $23.72 \pm 2.35$ & $\begin{array}{c}+0.62 \\
-0.51\end{array}$ & $21.28 \pm 2.19$ & $\begin{array}{c}+0.57 \\
-0.23\end{array}$ & $15.73 \pm 2.03$ & $\begin{array}{c}+0.00 \\
-0.56\end{array}$ & $16.20 \pm 2.09$ & $\begin{array}{c}+0.62 \\
-0.00\end{array}$ & $10.80 \pm 1.35$ & $\begin{array}{c}+0.84 \\
-0.08\end{array}$ \\
\hline 277.5 & $22.34 \pm 2.49$ & $\begin{array}{c}+0.00 \\
-1.12 \\
\end{array}$ & $25.82 \pm 2.63$ & $\begin{array}{c}+0.01 \\
-0.27\end{array}$ & $17.83 \pm 2.44$ & $\begin{array}{c}+0.17 \\
-0.63\end{array}$ & $15.41 \pm 2.24$ & $\begin{array}{c}+0.00 \\
-0.74 \\
\end{array}$ & $15.33 \pm 1.72$ & $\begin{array}{c}+0.61 \\
-0.00 \\
\end{array}$ \\
\hline 292.5 & $27.08 \pm 2.79$ & $\begin{array}{c}+0.24 \\
-0.03\end{array}$ & $22.20 \pm 2.66$ & $\begin{array}{c}+0.64 \\
-0.00\end{array}$ & $20.88 \pm 2.75$ & $\begin{array}{c}+0.17 \\
-0.59\end{array}$ & $15.15 \pm 2.64$ & $\begin{array}{c}+0.72 \\
-1.37\end{array}$ & $-8.57 \pm 2.61$ & $\begin{array}{c}+2.60 \\
-0.00\end{array}$ \\
\hline 307.5 & $29.04 \pm 2.88$ & $\begin{array}{c}+0.88 \\
-0.00\end{array}$ & $30.62 \pm 2.96$ & $\begin{array}{c}+0.17 \\
-0.78\end{array}$ & $13.92 \pm 3.09$ & $\begin{array}{c}+0.00 \\
-0.78\end{array}$ & $13.59 \pm 3.47$ & $\begin{array}{c}+0.79 \\
-1.11\end{array}$ & $18.45 \pm 3.45$ & $\begin{array}{c}+0.78 \\
-2.68\end{array}$ \\
\hline 322.5 & $31.78 \pm 3.07$ & $\begin{array}{c}+0.86 \\
-0.00\end{array}$ & $23.17 \pm 3.00$ & $\begin{array}{c}+0.99 \\
-0.21\end{array}$ & $27.48 \pm 4.02$ & $\begin{array}{c}+0.06 \\
-0.83\end{array}$ & $19.98 \pm 7.61$ & $\begin{array}{c}+1.76 \\
-1.56\end{array}$ & $25.91 \pm 16.59$ & $\begin{array}{l}+0.78 \\
-2.68\end{array}$ \\
\hline 337.5 & $33.98 \pm 3.16$ & $\begin{array}{c}+0.68 \\
-0.09 \\
\end{array}$ & $20.96 \pm 3.19$ & $\begin{array}{c}+0.21 \\
-0.06\end{array}$ & $33.01 \pm 6.03$ & $\begin{array}{c}+1.50 \\
-0.90 \\
\end{array}$ & $46.02 \pm 21.01$ & $\begin{array}{c}+11.24 \\
-0.00 \\
\end{array}$ & $-10.24 \pm 58.14$ & $\begin{array}{c}+3.85 \\
-9.73 \\
\end{array}$ \\
\hline 352.5 & $27.70 \pm 3.09$ & $\begin{array}{c}+0.50 \\
-0.00\end{array}$ & $28.83 \pm 3.74$ & $\begin{array}{c}+0.54 \\
-0.34\end{array}$ & $47.00 \pm 8.60$ & $\begin{array}{c}+0.84 \\
-0.43\end{array}$ & $29.00 \pm 33.68$ & $\begin{array}{c}+0.51 \\
-8.77\end{array}$ & $-23.46 \pm 32.33$ & $\begin{array}{c}+10.18 \\
-0.00\end{array}$ \\
\hline
\end{tabular}

Table D.1: Table of unpolarized extracted cross section values $\left(\mathrm{pb} / \mathrm{GeV}^{4}\right)$ for Kin481. The \pm values are the statistical errors, and the separated + and - are the systematic error introduced from the missing mass cut. The overall systematic uncertainties from table 6.4 are not represented here. 


\begin{tabular}{|c|c|c|c|c|c|c|c|c|c|c|}
\hline \multirow{2}{*}{$\begin{array}{c}\phi_{\gamma \gamma} \\
\text { (deg) }\end{array}$} & \multicolumn{2}{|c|}{$\begin{array}{c}\left\langle x_{B}\right\rangle=0.497 \\
\left\langle Q^{2}\right\rangle=4.497 \mathrm{GeV}^{2} \\
\left\langle t^{\prime}\right\rangle=-0.031 \mathrm{GeV}^{2}\end{array}$} & \multicolumn{2}{|c|}{$\begin{array}{c}\left\langle x_{B}\right\rangle=0.501 \\
\left\langle Q^{2}\right\rangle=4.528 \mathrm{GeV}^{2} \\
\left\langle t^{\prime}\right\rangle=-0.093 \mathrm{GeV}^{2}\end{array}$} & \multicolumn{2}{|c|}{$\begin{array}{c}\left\langle x_{B}\right\rangle=0.504 \\
\left\langle Q^{2}\right\rangle=4.558 \mathrm{GeV}^{2} \\
\left\langle t^{\prime}\right\rangle=-0.154 \mathrm{GeV}^{2}\end{array}$} & \multicolumn{2}{|c|}{$\begin{array}{c}\left\langle x_{B}\right\rangle=0.506 \\
\left\langle Q^{2}\right\rangle=4.573 \mathrm{GeV}^{2} \\
\left\langle t^{\prime}\right\rangle=-0.216 \mathrm{GeV}^{2}\end{array}$} & \multicolumn{2}{|c|}{$\begin{array}{c}\left\langle x_{B}\right\rangle=0.508 \\
\left\langle Q^{2}\right\rangle=4.593 \mathrm{GeV}^{2} \\
\left\langle t^{\prime}\right\rangle=-0.305 \mathrm{GeV}^{2}\end{array}$} \\
\hline & $3.92 \pm 0.36$ & $\begin{array}{l}+0.11 \\
-0.10\end{array}$ & $1.58 \pm 0.41$ & $\begin{array}{l}+0.01 \\
-0.05\end{array}$ & $1.70 \pm 0.76$ & $\begin{array}{l}+0.57 \\
-0.00\end{array}$ & $-0.67 \pm 1.03$ & $\begin{array}{l}+0.09 \\
-0.09\end{array}$ & $-2.76 \pm 1.36$ & $\begin{array}{l}+0.00 \\
-1.17\end{array}$ \\
\hline 22.5 & $4.41 \pm 0.38$ & $\begin{array}{l}+0.12 \\
-0.11\end{array}$ & $2.14 \pm 0.35$ & $\begin{array}{l}+0.02 \\
-0.07\end{array}$ & $2.83 \pm 0.62$ & $\begin{array}{l}+0.77 \\
-0.00\end{array}$ & $-0.49 \pm 0.94$ & $\begin{array}{l}+0.06 \\
-0.00\end{array}$ & $0.00 \pm 0.25$ & $\begin{array}{l}+0.00 \\
-0.00\end{array}$ \\
\hline 37.5 & $4.40 \pm 0.34$ & $\begin{array}{l}+0.11 \\
-0.12\end{array}$ & $2.91 \pm 0.34$ & $\begin{array}{l}+0.04 \\
-0.10\end{array}$ & $1.54 \pm 0.41$ & $\begin{array}{l}+0.28 \\
-0.00\end{array}$ & $1.33 \pm 0.60$ & $\begin{array}{l}+0.11 \\
-0.00\end{array}$ & $-0.40 \pm 0.94$ & $\begin{array}{l}+0.11 \\
-0.00\end{array}$ \\
\hline 52.5 & $3.78 \pm 0.34$ & $\begin{array}{l}+0.09 \\
-0.11\end{array}$ & $2.34 \pm 0.27$ & $\begin{array}{l}+0.04 \\
-0.08\end{array}$ & $1.55 \pm 0.29$ & $\begin{array}{l}+0.14 \\
-0.03\end{array}$ & $1.83 \pm 0.38$ & $\begin{array}{l}+0.07 \\
-0.03\end{array}$ & $0.75 \pm 0.39$ & $\begin{array}{l}+0.05 \\
-0.00\end{array}$ \\
\hline 67.5 & $4.50 \pm 0.34$ & $\begin{array}{l}+0.10 \\
-0.14\end{array}$ & $2.43 \pm 0.25$ & $\begin{array}{l}+0.05 \\
-0.08\end{array}$ & $1.95 \pm 0.25$ & $\begin{array}{l}+0.06 \\
-0.01\end{array}$ & $1.69 \pm 0.28$ & $\begin{array}{l}+0.01 \\
-0.03\end{array}$ & $0.72 \pm 0.24$ & $\begin{array}{l}+0.01 \\
-0.00\end{array}$ \\
\hline 82.5 & $4.47 \pm 0.34$ & $\begin{array}{l}+0.09 \\
-0.15\end{array}$ & $2.35 \pm 0.25$ & $\begin{array}{l}+0.05 \\
-0.08\end{array}$ & $2.20 \pm 0.25$ & $\begin{array}{l}+0.02 \\
-0.02\end{array}$ & $1.73 \pm 0.27$ & $\begin{array}{l}+0.01 \\
-0.07\end{array}$ & $1.11 \pm 0.20$ & $\begin{array}{l}+0.03 \\
-0.00\end{array}$ \\
\hline 97.5 & $4.09 \pm 0.34$ & $\begin{array}{l}+0.08 \\
-0.14\end{array}$ & $2.27 \pm 0.24$ & $\begin{array}{l}+0.05 \\
-0.06\end{array}$ & $1.85 \pm 0.23$ & $\begin{array}{l}+0.02 \\
-0.04\end{array}$ & $1.26 \pm 0.23$ & $\begin{array}{l}+0.04 \\
-0.05\end{array}$ & $1.33 \pm 0.19$ & $\begin{array}{l}+0.00 \\
-0.01\end{array}$ \\
\hline 112.5 & $3.71 \pm 0.32$ & $\begin{array}{l}+0.07 \\
-0.13\end{array}$ & $2.61 \pm 0.26$ & $\begin{array}{l}+0.05 \\
-0.06\end{array}$ & $1.97 \pm 0.23$ & $\begin{array}{l}+0.02 \\
-0.05\end{array}$ & $1.69 \pm 0.23$ & $\begin{array}{l}+0.00 \\
-0.06\end{array}$ & $1.56 \pm 0.16$ & $\begin{array}{l}+0.00 \\
-0.01\end{array}$ \\
\hline 127.5 & $3.67 \pm 0.31$ & $\begin{array}{l}+0.07 \\
-0.13\end{array}$ & $2.27 \pm 0.25$ & $\begin{array}{l}+0.03 \\
-0.04\end{array}$ & $2.01 \pm 0.23$ & $\begin{array}{l}+0.02 \\
-0.05\end{array}$ & $1.44 \pm 0.21$ & $\begin{array}{l}+0.00 \\
-0.04\end{array}$ & $1.37 \pm 0.15$ & $\begin{array}{l}+0.00 \\
-0.01\end{array}$ \\
\hline 142.5 & $3.31 \pm 0.29$ & $\begin{array}{l}+0.06 \\
-0.12\end{array}$ & $2.51 \pm 0.24$ & $\begin{array}{l}+0.03 \\
-0.04\end{array}$ & $2.00 \pm 0.22$ & $\begin{array}{l}+0.02 \\
-0.03\end{array}$ & $1.67 \pm 0.23$ & $\begin{array}{l}+0.00 \\
-0.03\end{array}$ & $1.25 \pm 0.14$ & $\begin{array}{l}+0.00 \\
-0.01\end{array}$ \\
\hline 157.5 & $3.42 \pm 0.28$ & $\begin{array}{l}+0.06 \\
-0.13\end{array}$ & $1.95 \pm 0.22$ & $\begin{array}{l}+0.02 \\
-0.02\end{array}$ & $2.08 \pm 0.22$ & $\begin{array}{l}+0.02 \\
-0.03\end{array}$ & $1.72 \pm 0.23$ & $\begin{array}{l}+0.00 \\
-0.02\end{array}$ & $1.46 \pm 0.16$ & $\begin{array}{l}+0.00 \\
-0.01\end{array}$ \\
\hline 172.5 & $2.93 \pm 0.26$ & $\begin{array}{l}+0.05 \\
-0.11\end{array}$ & $1.96 \pm 0.21$ & $\begin{array}{l}+0.03 \\
-0.02\end{array}$ & $1.84 \pm 0.22$ & $\begin{array}{l}+0.02 \\
-0.02\end{array}$ & $1.87 \pm 0.23$ & $\begin{array}{l}+0.01 \\
-0.02\end{array}$ & $1.51 \pm 0.17$ & $\begin{array}{l}+0.00 \\
-0.01\end{array}$ \\
\hline 187.5 & $3.33 \pm 0.27$ & $\begin{array}{l}+0.06 \\
-0.12 \\
\end{array}$ & $1.99 \pm 0.23$ & $\begin{array}{l}+0.03 \\
-0.02\end{array}$ & $2.37 \pm 0.24$ & $\begin{array}{l}+0.02 \\
-0.03\end{array}$ & $2.20 \pm 0.24$ & $\begin{array}{l}+0.01 \\
-0.02\end{array}$ & $1.49 \pm 0.16$ & $\begin{array}{l}+0.00 \\
-0.01\end{array}$ \\
\hline 202.5 & $3.27 \pm 0.30$ & $\begin{array}{l}+0.06 \\
-0.12\end{array}$ & $2.56 \pm 0.24$ & $\begin{array}{l}+0.03 \\
-0.03\end{array}$ & $1.65 \pm 0.22$ & $\begin{array}{l}+0.01 \\
-0.02\end{array}$ & $1.83 \pm 0.22$ & $\begin{array}{l}+0.00 \\
-0.02\end{array}$ & $1.63 \pm 0.16$ & $\begin{array}{l}+0.00 \\
-0.01\end{array}$ \\
\hline 217.5 & $2.87 \pm 0.27$ & $\begin{array}{l}+0.05 \\
-0.10\end{array}$ & $2.09 \pm 0.23$ & $\begin{array}{l}+0.02 \\
-0.03\end{array}$ & $1.57 \pm 0.21$ & $\begin{array}{l}+0.01 \\
-0.03\end{array}$ & $1.83 \pm 0.23$ & $\begin{array}{l}+0.00 \\
-0.03\end{array}$ & $1.69 \pm 0.15$ & $\begin{array}{l}+0.00 \\
-0.01\end{array}$ \\
\hline 232.5 & $3.29 \pm 0.30$ & $\begin{array}{l}+0.06 \\
-0.11\end{array}$ & $2.45 \pm 0.25$ & $\begin{array}{l}+0.04 \\
-0.05\end{array}$ & $2.00 \pm 0.23$ & $\begin{array}{l}+0.02 \\
-0.05\end{array}$ & $1.84 \pm 0.22$ & $\begin{array}{l}+0.00 \\
-0.05\end{array}$ & $1.40 \pm 0.14$ & $\begin{array}{l}+0.00 \\
-0.01\end{array}$ \\
\hline 247.5 & $3.65 \pm 0.32$ & $\begin{array}{l}+0.07 \\
-0.13\end{array}$ & $2.70 \pm 0.27$ & $\begin{array}{l}+0.05 \\
-0.06\end{array}$ & $1.92 \pm 0.24$ & $\begin{array}{l}+0.02 \\
-0.05\end{array}$ & $1.99 \pm 0.23$ & $\begin{array}{l}+0.00 \\
-0.07\end{array}$ & $1.37 \pm 0.15$ & $\begin{array}{l}+0.00 \\
-0.01\end{array}$ \\
\hline 262.5 & $4.32 \pm 0.35$ & $\begin{array}{l}+0.08 \\
-0.15\end{array}$ & $3.18 \pm 0.29$ & $\begin{array}{l}+0.06 \\
-0.09\end{array}$ & $2.26 \pm 0.25$ & $\begin{array}{l}+0.02 \\
-0.05\end{array}$ & $1.99 \pm 0.24$ & $\begin{array}{l}+0.00 \\
-0.08\end{array}$ & $1.23 \pm 0.16$ & $\begin{array}{l}+0.00 \\
-0.01\end{array}$ \\
\hline 277.5 & $4.34 \pm 0.35$ & $\begin{array}{l}+0.09 \\
-0.14\end{array}$ & $3.06 \pm 0.28$ & $\begin{array}{l}+0.07 \\
-0.09\end{array}$ & $2.19 \pm 0.25$ & $\begin{array}{l}+0.02 \\
-0.02\end{array}$ & $1.93 \pm 0.25$ & $\begin{array}{l}+0.01 \\
-0.07\end{array}$ & $1.54 \pm 0.18$ & $\begin{array}{l}+0.00 \\
-0.00\end{array}$ \\
\hline 292.5 & $4.60 \pm 0.35$ & $\begin{array}{l}+0.10 \\
-0.11\end{array}$ & $3.37 \pm 0.029$ & $\begin{array}{l}+0.07 \\
-0.00\end{array}$ & $2.40 \pm 0.27$ & $\begin{array}{l}+0.08 \\
-0.01\end{array}$ & $1.33 \pm 0.24$ & $\begin{array}{l}+0.01 \\
-0.03\end{array}$ & $0.90 \pm 0.21$ & $\begin{array}{l}+0.02 \\
-0.00\end{array}$ \\
\hline 307.5 & $3.82 \pm 0.33$ & $\begin{array}{l}+0.09 \\
-0.11\end{array}$ & $2.51 \pm 0.27$ & $\begin{array}{l}+0.05 \\
-0.08\end{array}$ & $2.49 \pm 0.30$ & $\begin{array}{l}+0.23 \\
-0.00\end{array}$ & $1.83 \pm 0.37$ & $\begin{array}{l}+0.07 \\
-0.00\end{array}$ & $0.81 \pm 0.33$ & $\begin{array}{l}+0.05 \\
-0.00\end{array}$ \\
\hline 322.5 & $4.11 \pm 0.34$ & $\begin{array}{l}+0.10 \\
-0.11\end{array}$ & $2.63 \pm 0.32$ & $\begin{array}{l}+0.04 \\
-0.09\end{array}$ & $1.56 \pm 0.42$ & $\begin{array}{l}+0.28 \\
-0.00\end{array}$ & $0.97 \pm 0.62$ & $\begin{array}{l}+0.08 \\
-0.00\end{array}$ & $3.56 \pm 1.35$ & $\begin{array}{l}+0.94 \\
-0.00\end{array}$ \\
\hline 337.5 & $4.22 \pm 0.36$ & $\begin{array}{l}+0.11 \\
-0.10\end{array}$ & $2.92 \pm 0.40$ & $\begin{array}{l}+0.03 \\
-0.10\end{array}$ & $1.50 \pm 0.56$ & $\begin{array}{l}+0.41 \\
-0.00\end{array}$ & $0.97 \pm 0.99$ & $\begin{array}{l}+0.11 \\
-0.00\end{array}$ & $-0.21 \pm 0.19$ & $\begin{array}{l}+0.00 \\
-0.31\end{array}$ \\
\hline 352.5 & $3.92 \pm 0.35$ & $\begin{array}{l}+0.11 \\
-0.10\end{array}$ & $2.52 \pm 0.42$ & $\begin{array}{l}+0.01 \\
-0.08\end{array}$ & $1.18 \pm 0.77$ & $\begin{array}{l}+0.39 \\
-0.00\end{array}$ & $-0.57 \pm 1.17$ & $\begin{array}{l}+0.08 \\
-0.00\end{array}$ & $-2.50 \pm 1.43$ & $\begin{array}{l}+0.00 \\
-1.06\end{array}$ \\
\hline
\end{tabular}

Table D.2: Table of unpolarized extracted cross section values $\left(\mathrm{pb} / \mathrm{GeV}^{4}\right)$ for Kin482. The \pm values are the statistical errors, and the separated + and - are the systematic error introduced from the missing mass cut. The overall systematic uncertainties from table 6.4 are not represented here. 


\begin{tabular}{|c|c|c|c|c|c|c|c|c|c|c|}
\hline \multirow{2}{*}{$\begin{array}{c}\begin{array}{l}\phi_{\gamma \gamma} \\
(\mathrm{deg})\end{array} \\
7.5\end{array}$} & \multicolumn{2}{|c|}{$\begin{array}{c}\left\langle x_{B}\right\rangle=0.483 \\
\left\langle Q^{2}\right\rangle=5.331 \mathrm{GeV}^{2} \\
\left\langle t^{\prime}\right\rangle=-0.030 \mathrm{GeV}^{2}\end{array}$} & \multicolumn{2}{|c|}{$\begin{array}{c}\left\langle x_{B}\right\rangle=0.483 \\
\left\langle Q^{2}\right\rangle=5.339 \mathrm{GeV}^{2} \\
\left\langle t^{\prime}\right\rangle=-0.088 \mathrm{GeV}^{2}\end{array}$} & \multicolumn{2}{|c|}{$\begin{array}{c}\left\langle x_{B}\right\rangle=0.485 \\
\left\langle Q^{2}\right\rangle=5.360 \mathrm{GeV}^{2} \\
\left\langle t^{\prime}\right\rangle=-0.147 \mathrm{GeV}^{2}\end{array}$} & \multicolumn{2}{|c|}{$\begin{array}{c}\left\langle x_{B}\right\rangle=0.486 \\
\left\langle Q^{2}\right\rangle=5.371 \mathrm{GeV}^{2} \\
\left\langle t^{\prime}\right\rangle=-0.206 \mathrm{GeV}^{2}\end{array}$} & \multicolumn{2}{|c|}{$\begin{array}{c}\left\langle x_{B}\right\rangle=0.486 \\
\left\langle Q^{2}\right\rangle=5.379 \mathrm{GeV}^{2} \\
\left\langle t^{\prime}\right\rangle=-0.291 \mathrm{GeV}^{2}\end{array}$} \\
\hline & $4.13 \pm 0.32$ & $\begin{array}{c}+0.10 \\
-0.19\end{array}$ & $2.78 \pm 0.34$ & $\begin{array}{c}+0.08 \\
-0.18\end{array}$ & $1.87 \pm 0.59$ & $\begin{array}{c}+0.02 \\
-0.04\end{array}$ & $-0.71 \pm 1.41$ & $\begin{array}{c}+0.05 \\
-0.15\end{array}$ & $-0.74 \pm 2.94$ & $\begin{array}{c}+0.73 \\
-0.28\end{array}$ \\
\hline 22.5 & $3.11 \pm 0.29$ & $\begin{array}{c}+0.07 \\
-0.14\end{array}$ & $2.63 \pm 0.31$ & $\begin{array}{l}+0.07 \\
-0.16\end{array}$ & $2.38 \pm 0.44$ & $\begin{array}{l}+0.03 \\
-0.06\end{array}$ & $0.92 \pm 0.70$ & $\begin{array}{l}+0.05 \\
-0.16\end{array}$ & $-1.45 \pm 1.56$ & $\begin{array}{l}+0.10 \\
-0.05\end{array}$ \\
\hline 37.5 & $3.38 \pm 0.28$ & $\begin{array}{l}+0.06 \\
-0.16\end{array}$ & $2.48 \pm 0.26$ & $\begin{array}{l}+0.06 \\
-0.14\end{array}$ & $2.01 \pm 0.31$ & $\begin{array}{l}+0.02 \\
-0.07\end{array}$ & $1.55 \pm 0.43$ & $\begin{array}{l}+0.05 \\
-0.16\end{array}$ & $-1.01 \pm 0.52$ & $\begin{array}{l}+0.03 \\
-0.02\end{array}$ \\
\hline 52.5 & $3.64 \pm 0.30$ & $\begin{array}{l}+0.05 \\
-0.18\end{array}$ & $2.50 \pm 0.25$ & $\begin{array}{l}+0.06 \\
-0.12\end{array}$ & $1.81 \pm 0.25$ & $\begin{array}{c}+0.02 \\
-0.07\end{array}$ & $1.61 \pm 0.30$ & $\begin{array}{l}+0.01 \\
-0.06\end{array}$ & $1.00 \pm 0.28$ & $\begin{array}{l}+0.00 \\
-0.02\end{array}$ \\
\hline 67.5 & $3.19 \pm 0.27$ & $\begin{array}{l}+0.03 \\
-0.15\end{array}$ & $2.29 \pm 0.23$ & $\begin{array}{l}+0.05 \\
-0.11\end{array}$ & $2.23 \pm 0.22$ & $\begin{array}{c}+0.02 \\
-0.10\end{array}$ & $1.85 \pm 0.22$ & $\begin{array}{l}+0.00 \\
-0.02\end{array}$ & $1.14 \pm 0.19$ & $\begin{array}{l}+0.00 \\
-0.05\end{array}$ \\
\hline 82.5 & $3.06 \pm 0.25$ & $\begin{array}{l}+0.02 \\
-0.14\end{array}$ & $2.20 \pm 0.23$ & $\begin{array}{l}+0.04 \\
-0.10\end{array}$ & $1.57 \pm 0.20$ & $\begin{array}{l}+0.02 \\
-0.07\end{array}$ & $1.52 \pm 0.20$ & $\begin{array}{l}+0.04 \\
-0.04\end{array}$ & $1.29 \pm 0.16$ & $\begin{array}{l}+0.00 \\
-0.08\end{array}$ \\
\hline 97.5 & $2.71 \pm 0.25$ & $\begin{array}{l}+0.02 \\
-0.12\end{array}$ & $1.90 \pm 0.20$ & $\begin{array}{l}+0.03 \\
-0.08\end{array}$ & $1.77 \pm 0.19$ & $\begin{array}{l}+0.02 \\
-0.07\end{array}$ & $1.36 \pm 0.18$ & $\begin{array}{l}+0.04 \\
-0.04\end{array}$ & $0.96 \pm 0.14$ & $\begin{array}{l}+0.00 \\
-0.06\end{array}$ \\
\hline 112.5 & $3.03 \pm 0.25$ & $\begin{array}{l}+0.02 \\
-0.12\end{array}$ & $1.61 \pm 0.18$ & $\begin{array}{l}+0.03 \\
-0.07\end{array}$ & $1.40 \pm 0.17$ & $\begin{array}{l}+0.02 \\
-0.05\end{array}$ & $1.00 \pm 0.16$ & $\begin{array}{l}+0.02 \\
-0.04\end{array}$ & $0.92 \pm 0.12$ & $\begin{array}{l}+0.01 \\
-0.05\end{array}$ \\
\hline 127.5 & $2.56 \pm 0.22$ & $\begin{array}{l}+0.03 \\
-0.10\end{array}$ & $1.88 \pm 0.18$ & $\begin{array}{l}+0.03 \\
-0.08\end{array}$ & $1.27 \pm 0.15$ & $\begin{array}{l}+0.02 \\
-0.04\end{array}$ & $1.76 \pm 0.18$ & $\begin{array}{l}+0.01 \\
-0.07\end{array}$ & $1.01 \pm 0.10$ & $\begin{array}{l}+0.01 \\
-0.05\end{array}$ \\
\hline 142.5 & $2.32 \pm 0.20$ & $\begin{array}{l}+0.03 \\
-0.08\end{array}$ & $1.77 \pm 0.17$ & $\begin{array}{l}+0.03 \\
-0.08\end{array}$ & $1.40 \pm 0.16$ & $\begin{array}{l}+0.02 \\
-0.03\end{array}$ & $1.44 \pm 0.16$ & $\begin{array}{l}+0.00 \\
-0.06\end{array}$ & $0.87 \pm 0.10$ & $\begin{array}{l}+0.01 \\
-0.03\end{array}$ \\
\hline 157.5 & $2.70 \pm 0.21$ & $\begin{array}{l}+0.04 \\
-0.08\end{array}$ & $1.41 \pm 0.16$ & $\begin{array}{l}+0.02 \\
-0.06\end{array}$ & $1.61 \pm 0.17$ & $\begin{array}{l}+0.02 \\
-0.02\end{array}$ & $1.30 \pm 0.16$ & $\begin{array}{l}+0.00 \\
-0.05\end{array}$ & $0.90 \pm 0.10$ & $\begin{array}{r}+0.01 \\
-0.02\end{array}$ \\
\hline 172.5 & $2.31 \pm 0.20$ & $\begin{array}{l}+0.04 \\
-0.07\end{array}$ & $1.63 \pm 0.17$ & $\begin{array}{l}+0.03 \\
-0.07\end{array}$ & $1.40 \pm 0.16$ & $\begin{array}{l}+0.02 \\
-0.01\end{array}$ & $1.44 \pm 0.16$ & $\begin{array}{l}+0.00 \\
-0.06\end{array}$ & $0.86 \pm 0.10$ & $\begin{array}{l}+0.01 \\
-0.02\end{array}$ \\
\hline 187.5 & $2.14 \pm 0.19$ & $\begin{array}{c}+0.03 \\
-0.08\end{array}$ & $1.57 \pm 0.17$ & $\begin{array}{l}+0.03 \\
-0.07\end{array}$ & $1.40 \pm 0.16$ & $\begin{array}{c}+0.02 \\
-0.01\end{array}$ & $1.02 \pm 0.15$ & $\begin{array}{l}+0.00 \\
-0.04\end{array}$ & $0.98 \pm 0.11$ & $\begin{array}{l}+0.01 \\
-0.02\end{array}$ \\
\hline 202.5 & $2.60 \pm 0.21$ & $\begin{array}{l}+0.04 \\
-0.08\end{array}$ & $1.85 \pm 0.18$ & $\begin{array}{l}+0.03 \\
-0.08\end{array}$ & $1.33 \pm 0.15$ & $\begin{array}{l}+0.02 \\
-0.02\end{array}$ & $1.06 \pm 0.15$ & $\begin{array}{l}+0.00 \\
-0.04\end{array}$ & $1.15 \pm 0.11$ & $\begin{array}{l}+0.01 \\
-0.03\end{array}$ \\
\hline 217.5 & $2.34 \pm 0.20$ & $\begin{array}{l}+0.03 \\
-0.08\end{array}$ & $1.41 \pm 0.15$ & $\begin{array}{l}+0.02 \\
-0.06\end{array}$ & $1.43 \pm 0.16$ & $\begin{array}{l}+0.02 \\
-0.03\end{array}$ & $1.18 \pm 0.15$ & $\begin{array}{l}+0.00 \\
-0.05\end{array}$ & $0.71 \pm 0.10$ & $\begin{array}{l}+0.01 \\
-0.02\end{array}$ \\
\hline 232.5 & $2.57 \pm 0.22$ & $\begin{array}{l}+0.03 \\
-0.10\end{array}$ & $1.97 \pm 0.19$ & $\begin{array}{l}+0.03 \\
-0.08\end{array}$ & $1.42 \pm 0.17$ & $\begin{array}{l}+0.02 \\
-0.04\end{array}$ & $1.20 \pm 0.17$ & $\begin{array}{l}+0.00 \\
-0.05\end{array}$ & $0.92 \pm 0.10$ & $\begin{array}{l}+0.01 \\
-0.04\end{array}$ \\
\hline 247.5 & $2.58 \pm 0.23$ & $\begin{array}{l}+0.02 \\
-0.11\end{array}$ & $2.32 \pm 0.21$ & $\begin{array}{l}+0.04 \\
-0.10\end{array}$ & $1.51 \pm 0.17$ & $\begin{array}{l}+0.02 \\
-0.05\end{array}$ & $1.17 \pm 0.16$ & $\begin{array}{l}+0.03 \\
-0.04\end{array}$ & $1.00 \pm 0.11$ & $\begin{array}{l}+0.01 \\
-0.06\end{array}$ \\
\hline 262.5 & $3.27 \pm 0.27$ & $\begin{array}{l}+0.02 \\
-0.15\end{array}$ & $2.42 \pm 0.22$ & $\begin{array}{l}+0.04 \\
-0.10\end{array}$ & $2.07 \pm 0.21$ & $\begin{array}{l}+0.02 \\
-0.08\end{array}$ & $1.55 \pm 0.19$ & $\begin{array}{l}+0.05 \\
-0.05\end{array}$ & $1.21 \pm 0.13$ & $\begin{array}{l}+0.01 \\
-0.08\end{array}$ \\
\hline 277.5 & $3.41 \pm 0.27$ & $\begin{array}{l}+0.03 \\
-0.16\end{array}$ & $2.46 \pm 0.23$ & $\begin{array}{l}+0.05 \\
-0.11\end{array}$ & $1.92 \pm 0.22$ & $\begin{array}{c}+0.02 \\
-0.08\end{array}$ & $1.38 \pm 0.20$ & $\begin{array}{l}+0.03 \\
-0.03\end{array}$ & $1.17 \pm 0.14$ & $\begin{array}{l}+0.00 \\
-0.07\end{array}$ \\
\hline 292.5 & $3.32 \pm 0.27$ & $\begin{array}{c}+0.03 \\
-0.16\end{array}$ & $2.62 \pm 0.24$ & $\begin{array}{c}+0.06 \\
-0.12 \\
\end{array}$ & $2.06 \pm 0.22$ & $\begin{array}{c}+0.02 \\
-0.09\end{array}$ & $1.81 \pm 0.23$ & $\begin{array}{l}+0.05 \\
-0.02 \\
\end{array}$ & $1.55 \pm 0.18$ & $\begin{array}{l}+0.00 \\
-0.07 \\
\end{array}$ \\
\hline 307.5 & $3.58 \pm 0.29$ & $\begin{array}{l}+0.05 \\
-0.17\end{array}$ & $2.64 \pm 0.27$ & $\begin{array}{c}+0.06 \\
-0.13\end{array}$ & $1.78 \pm 0.24$ & $\begin{array}{c}+0.02 \\
-0.07\end{array}$ & $1.86 \pm 0.28$ & $\begin{array}{l}+0.01 \\
-0.07\end{array}$ & $1.36 \pm 0.26$ & $\begin{array}{l}+0.01 \\
-0.02\end{array}$ \\
\hline 322.5 & $4.07 \pm 0.28$ & $\begin{array}{l}+0.07 \\
-0.19\end{array}$ & $3.06 \pm 0.27$ & $\begin{array}{l}+0.08 \\
-0.17\end{array}$ & $1.97 \pm 0.34$ & $\begin{array}{l}+0.02 \\
-0.07\end{array}$ & $1.17 \pm 0.45$ & $\begin{array}{l}+0.04 \\
-0.12\end{array}$ & $1.79 \pm 0.62$ & $\begin{array}{l}+0.06 \\
-0.04\end{array}$ \\
\hline 337.5 & $4.10 \pm 0.31$ & $\begin{array}{c}+0.09 \\
-0.19\end{array}$ & $2.60 \pm 0.30$ & $\begin{array}{l}+0.07 \\
-0.16\end{array}$ & $2.22 \pm 0.45$ & $\begin{array}{l}+0.02 \\
-0.06\end{array}$ & $2.88 \pm 0.95$ & $\begin{array}{l}+0.16 \\
-0.49\end{array}$ & $0.00 \pm 1.59$ & $\begin{array}{c}+0.00 \\
-0.00\end{array}$ \\
\hline 352.5 & $3.57 \pm 0.31$ & $\begin{array}{l}+0.08 \\
-0.16\end{array}$ & $2.67 \pm 0.35$ & $\begin{array}{l}+0.08 \\
-0.17\end{array}$ & $2.16 \pm 0.64$ & $\begin{array}{l}+0.02 \\
-0.05\end{array}$ & $-1.92 \pm 1.55$ & $\begin{array}{l}+0.13 \\
-0.42\end{array}$ & $-1.22 \pm 1.20$ & $\begin{array}{l}+0.12 \\
-0.05\end{array}$ \\
\hline
\end{tabular}

Table D.3: Table of unpolarized extracted cross section values $\left(\mathrm{pb} / \mathrm{GeV}^{4}\right)$ for Kin483. The \pm values are the statistical errors, and the separated + and - are the systematic error introduced from the missing mass cut. The overall systematic uncertainties from table 6.4 are not represented here. 


\begin{tabular}{|c|c|c|c|c|c|c|c|c|c|c|}
\hline \multirow{2}{*}{$\begin{array}{c}\phi_{\gamma \gamma} \\
\text { (deg) }\end{array}$} & \multicolumn{2}{|c|}{$\begin{array}{c}\left\langle x_{B}\right\rangle=0.494 \\
\left\langle Q^{2}\right\rangle=7.044 \mathrm{GeV}^{2} \\
\left\langle t^{\prime}\right\rangle=-0.043 \mathrm{GeV}^{2}\end{array}$} & \multicolumn{2}{|c|}{$\begin{array}{c}\left\langle x_{B}\right\rangle=0.498 \\
\left\langle Q^{2}\right\rangle=7.093 \mathrm{GeV}^{2} \\
\left\langle t^{\prime}\right\rangle=-0.127 \mathrm{GeV}^{2}\end{array}$} & \multicolumn{2}{|c|}{$\begin{array}{c}\left\langle x_{B}\right\rangle=0.499 \\
\left\langle Q^{2}\right\rangle=7.115 \mathrm{GeV}^{2} \\
\left\langle t^{\prime}\right\rangle=-0.212 \mathrm{GeV}^{2}\end{array}$} & \multicolumn{2}{|c|}{$\begin{array}{c}\left\langle x_{B}\right\rangle=0.499 \\
\left\langle Q^{2}\right\rangle=7.106 \mathrm{GeV}^{2} \\
\left\langle t^{\prime}\right\rangle=-0.297 \mathrm{GeV}^{2}\end{array}$} & \multicolumn{2}{|c|}{$\begin{array}{c}\left\langle x_{B}\right\rangle=0.498 \\
\left\langle Q^{2}\right\rangle=7.102 \mathrm{GeV}^{2} \\
\left\langle t^{\prime}\right\rangle=-0.457 \mathrm{GeV}^{2}\end{array}$} \\
\hline & $3.06 \pm 0.20$ & $\begin{array}{c}+0.00 \\
-0.06\end{array}$ & $2.04 \pm 0.32$ & $\begin{array}{l}+0.04 \\
-0.01\end{array}$ & $0.00 \pm 1.17$ & $\begin{array}{c}+0.00 \\
-0.00\end{array}$ & $-18.01 \pm 1.77$ & $\begin{array}{l}+1.38 \\
-2.83\end{array}$ & $0.00 \pm 0.00$ & $\begin{array}{c}+0.00 \\
-0.00\end{array}$ \\
\hline 22.5 & $3.44 \pm 0.21$ & $\begin{array}{l}+0.00 \\
-0.06\end{array}$ & $1.89 \pm 0.26$ & $\begin{array}{l}+0.02 \\
-0.01\end{array}$ & $1.52 \pm 0.71$ & $\begin{array}{l}+0.04 \\
-0.35\end{array}$ & $-3.26 \pm 3.20$ & $\begin{array}{l}+0.21 \\
-0.45\end{array}$ & $0.00 \pm 0.00$ & $\begin{array}{l}+0.00 \\
-0.00\end{array}$ \\
\hline 37.5 & $3.15 \pm 0.19$ & $\begin{array}{l}+0.00 \\
-0.05\end{array}$ & $1.65 \pm 0.20$ & $\begin{array}{l}+0.02 \\
-0.00\end{array}$ & $0.56 \pm 0.33$ & $\begin{array}{l}+0.01 \\
-0.10\end{array}$ & $0.56 \pm 0.68$ & $\begin{array}{l}+0.02 \\
-0.06\end{array}$ & $0.60 \pm 1.55$ & $\begin{array}{l}+0.07 \\
-0.00\end{array}$ \\
\hline 52.5 & $2.89 \pm 0.18$ & $\begin{array}{l}+0.00 \\
-0.03\end{array}$ & $2.03 \pm 0.16$ & $\begin{array}{l}+0.02 \\
-0.00\end{array}$ & $1.59 \pm 0.18$ & $\begin{array}{l}+0.02 \\
-0.17\end{array}$ & $0.55 \pm 0.27$ & $\begin{array}{l}+0.01 \\
-0.04\end{array}$ & $0.52 \pm 0.18$ & $\begin{array}{l}+0.03 \\
-0.00\end{array}$ \\
\hline 67.5 & $2.70 \pm 0.18$ & $\begin{array}{l}+0.00 \\
-0.03\end{array}$ & $1.89 \pm 0.15$ & $\begin{array}{l}+0.01 \\
-0.00\end{array}$ & $1.64 \pm 0.14$ & $\begin{array}{l}+0.01 \\
-0.08\end{array}$ & $0.99 \pm 0.16$ & $\begin{array}{l}+0.01 \\
-0.04\end{array}$ & $0.59 \pm 0.08$ & $\begin{array}{l}+0.02 \\
-0.00\end{array}$ \\
\hline 82.5 & $2.31 \pm 0.17$ & $\begin{array}{l}+0.00 \\
-0.02\end{array}$ & $1.91 \pm 0.14$ & $\begin{array}{l}+0.06 \\
-0.00\end{array}$ & $1.33 \pm 0.12$ & $\begin{array}{l}+0.06 \\
-0.02\end{array}$ & $1.00 \pm 0.16$ & $\begin{array}{l}+0.00 \\
-0.02\end{array}$ & $0.72 \pm 0.06$ & $\begin{array}{l}+0.01 \\
-0.00\end{array}$ \\
\hline 97.5 & $2.39 \pm 0.16$ & $\begin{array}{l}+0.00 \\
-0.03\end{array}$ & $1.43 \pm 0.12$ & $\begin{array}{l}+0.05 \\
-0.00\end{array}$ & $1.29 \pm 0.11$ & $\begin{array}{l}+0.01 \\
-0.00\end{array}$ & $0.77 \pm 0.13$ & $\begin{array}{l}+0.00 \\
-0.01\end{array}$ & $0.62 \pm 0.05$ & $\begin{array}{l}+0.00 \\
-0.00\end{array}$ \\
\hline 112.5 & $2.21 \pm 0.15$ & $\begin{array}{l}+0.00 \\
-0.03\end{array}$ & $1.80 \pm 0.13$ & $\begin{array}{l}+0.01 \\
-0.01\end{array}$ & $1.04 \pm 0.10$ & $\begin{array}{l}+0.01 \\
-0.00\end{array}$ & $0.83 \pm 0.13$ & $\begin{array}{l}+0.00 \\
-0.01\end{array}$ & $0.57 \pm 0.05$ & $\begin{array}{l}+0.00 \\
-0.00\end{array}$ \\
\hline 127.5 & $2.12 \pm 0.14$ & $\begin{array}{l}+0.00 \\
-0.04\end{array}$ & $1.56 \pm 0.13$ & $\begin{array}{l}+0.00 \\
-0.01\end{array}$ & $0.98 \pm 0.10$ & $\begin{array}{l}+0.00 \\
-0.00\end{array}$ & $0.78 \pm 0.12$ & $\begin{array}{l}+0.00 \\
-0.01\end{array}$ & $0.55 \pm 0.04$ & $\begin{array}{l}+0.00 \\
-0.00\end{array}$ \\
\hline 142.5 & $2.28 \pm 0.14$ & $\begin{array}{l}+0.00 \\
-0.05\end{array}$ & $1.48 \pm 0.12$ & $\begin{array}{l}+0.00 \\
-0.01\end{array}$ & $1.10 \pm 0.11$ & $\begin{array}{l}+0.01 \\
-0.01\end{array}$ & $0.68 \pm 0.12$ & $\begin{array}{l}+0.00 \\
-0.01\end{array}$ & $0.57 \pm 0.04$ & $\begin{array}{l}+0.00 \\
-0.00\end{array}$ \\
\hline 157.5 & $2.04 \pm 0.14$ & $\begin{array}{l}+0.00 \\
-0.06\end{array}$ & $1.30 \pm 0.12$ & $\begin{array}{l}+0.00 \\
-0.01\end{array}$ & $1.03 \pm 0.10$ & $\begin{array}{l}+0.01 \\
-0.02\end{array}$ & $0.71 \pm 0.11$ & $\begin{array}{l}+0.00 \\
-0.00\end{array}$ & $0.54 \pm 0.04$ & $\begin{array}{l}+0.01 \\
-0.00\end{array}$ \\
\hline 172.5 & $1.95 \pm 0.14$ & $\begin{array}{l}+0.00 \\
-0.06\end{array}$ & $1.26 \pm 0.11$ & $\begin{array}{l}+0.00 \\
-0.01\end{array}$ & $0.99 \pm 0.10$ & $\begin{array}{l}+0.01 \\
-0.03\end{array}$ & $0.91 \pm 0.12$ & $\begin{array}{l}+0.01 \\
-0.01\end{array}$ & $0.56 \pm 0.04$ & $\begin{array}{l}+0.01 \\
-0.00\end{array}$ \\
\hline 187.5 & $2.02 \pm 0.14$ & $\begin{array}{l}+0.00 \\
-0.06\end{array}$ & $1.38 \pm 0.11$ & $\begin{array}{l}+0.00 \\
-0.01\end{array}$ & $1.01 \pm 0.10$ & $\begin{array}{l}+0.01 \\
-0.03\end{array}$ & $0.66 \pm 0.11$ & $\begin{array}{l}+0.00 \\
-0.00\end{array}$ & $0.54 \pm 0.05$ & $\begin{array}{l}+0.01 \\
-0.00\end{array}$ \\
\hline 202.5 & $2.17 \pm 0.14$ & $\begin{array}{l}+0.00 \\
-0.06\end{array}$ & $1.26 \pm 0.11$ & $\begin{array}{l}+0.00 \\
-0.01\end{array}$ & $1.01 \pm 0.11$ & $\begin{array}{l}+0.01 \\
-0.02\end{array}$ & $0.90 \pm 0.13$ & $\begin{array}{l}+0.00 \\
-0.01\end{array}$ & $0.52 \pm 0.04$ & $\begin{array}{l}+0.01 \\
-0.00\end{array}$ \\
\hline 217.5 & $2.17 \pm 0.14$ & $\begin{array}{l}+0.00 \\
-0.05\end{array}$ & $1.54 \pm 0.13$ & $\begin{array}{l}+0.00 \\
-0.01\end{array}$ & $1.06 \pm 0.11$ & $\begin{array}{l}+0.01 \\
-0.01\end{array}$ & $0.81 \pm 0.12$ & $\begin{array}{l}+0.00 \\
-0.01\end{array}$ & $0.58 \pm 0.04$ & $\begin{array}{l}+0.00 \\
-0.00\end{array}$ \\
\hline 232.5 & $2.31 \pm 0.15$ & $\begin{array}{l}+0.00 \\
-0.04\end{array}$ & $1.46 \pm 0.12$ & $\begin{array}{l}+0.00 \\
-0.01\end{array}$ & $1.23 \pm 0.11$ & $\begin{array}{l}+0.01 \\
-0.00\end{array}$ & $1.03 \pm 0.13$ & $\begin{array}{l}+0.00 \\
-0.01\end{array}$ & $0.59 \pm 0.05$ & $\begin{array}{l}+0.00 \\
-0.00\end{array}$ \\
\hline 247.5 & $2.17 \pm 0.15$ & $\begin{array}{l}+0.00 \\
-0.03\end{array}$ & $1.67 \pm 0.13$ & $\begin{array}{l}+0.00 \\
-0.01\end{array}$ & $1.01 \pm 0.11$ & $\begin{array}{c}+0.01 \\
-0.00\end{array}$ & $0.93 \pm 0.13$ & $\begin{array}{l}+0.00 \\
-0.01\end{array}$ & $0.60 \pm 0.05$ & $\begin{array}{l}+0.00 \\
-0.00\end{array}$ \\
\hline 262.5 & $2.41 \pm 0.017$ & $\begin{array}{l}+0.00 \\
-0.03\end{array}$ & $1.43 \pm 0.13$ & $\begin{array}{l}+0.00 \\
-0.00\end{array}$ & $1.00 \pm 0.11$ & $\begin{array}{l}+0.01 \\
-0.00\end{array}$ & $1.05 \pm 0.16$ & $\begin{array}{l}+0.00 \\
-0.01\end{array}$ & $0.72 \pm 0.05$ & $\begin{array}{l}+0.00 \\
-0.00\end{array}$ \\
\hline 277.5 & $2.81 \pm 0.18$ & $\begin{array}{l}+0.00 \\
-0.03\end{array}$ & $1.62 \pm 0.14$ & $\begin{array}{l}+0.00 \\
-0.00\end{array}$ & $1.40 \pm 0.13$ & $\begin{array}{l}+0.01 \\
-0.02\end{array}$ & $0.87 \pm 0.15$ & $\begin{array}{l}+0.00 \\
-0.02\end{array}$ & $0.66 \pm 0.06$ & $\begin{array}{l}+0.01 \\
-0.00\end{array}$ \\
\hline 292.5 & $2.76 \pm 0.17$ & $\begin{array}{l}+0.00 \\
-0.03\end{array}$ & $1.84 \pm 0.14$ & $\begin{array}{l}+0.01 \\
-0.00\end{array}$ & $1.33 \pm 0.14$ & $\begin{array}{l}+0.01 \\
-0.01\end{array}$ & $1.13 \pm 0.17$ & $\begin{array}{l}+0.01 \\
-0.04\end{array}$ & $0.68 \pm 0.07$ & $\begin{array}{l}+0.02 \\
-0.00\end{array}$ \\
\hline 307.5 & $3.34 \pm 0.20$ & $\begin{array}{l}+0.00 \\
-0.04\end{array}$ & $2.19 \pm 0.17$ & $\begin{array}{l}+0.02 \\
-0.00\end{array}$ & $1.85 \pm 0.18$ & $\begin{array}{c}+0.02 \\
-0.20\end{array}$ & $1.15 \pm 0.29$ & $\begin{array}{l}+0.03 \\
-0.08\end{array}$ & $0.70 \pm 0.18$ & $\begin{array}{l}+0.04 \\
-0.00\end{array}$ \\
\hline 322.5 & $3.35 \pm 0.20$ & $\begin{array}{l}+0.00 \\
-0.05\end{array}$ & $2.25 \pm 0.21$ & $\begin{array}{l}+0.03 \\
-0.01\end{array}$ & $1.39 \pm 0.29$ & $\begin{array}{l}+0.02 \\
-0.24\end{array}$ & $0.52 \pm 0.82$ & $\begin{array}{l}+0.02 \\
-0.05\end{array}$ & $0.00 \pm 1.56$ & $\begin{array}{l}+0.00 \\
-0.00\end{array}$ \\
\hline 337.5 & $3.81 \pm 0.22$ & $\begin{array}{l}+0.00 \\
-0.07\end{array}$ & $3.07 \pm 0.30$ & $\begin{array}{l}+0.05 \\
-0.01\end{array}$ & $1.46 \pm 0.67$ & $\begin{array}{l}+0.03 \\
-0.34\end{array}$ & $-0.23 \pm 2.26$ & $\begin{array}{l}+0.15 \\
-0.32\end{array}$ & $-8.70 \pm 8.54$ & $\begin{array}{l}+1.95 \\
-0.00\end{array}$ \\
\hline 352.5 & $3.40 \pm 0.23$ & $\begin{array}{l}+0.00 \\
-0.07\end{array}$ & $2.16 \pm 0.33$ & $\begin{array}{l}+0.04 \\
-0.01\end{array}$ & $0.35 \pm 0.60$ & $\begin{array}{l}+0.01 \\
-0.10\end{array}$ & $0.00 \pm 0.00$ & $\begin{array}{l}+0.00 \\
-0.00\end{array}$ & $0.33 \pm 0.00$ & $\begin{array}{l}+0.11 \\
-0.00\end{array}$ \\
\hline
\end{tabular}

Table D.4: Table of unpolarized extracted cross section values $\left(\mathrm{pb} / \mathrm{GeV}^{4}\right)$ for Kin484. The \pm values are the statistical errors, and the separated + and - are the systematic error introduced from the missing mass cut. The overall systematic uncertainties from table 6.4 are not represented here. 
Appendix E

\title{
Tables of Polarized DVCS cross sections
}

\author{
E.1 Polarized Cross Sections
}




\begin{tabular}{|c|c|c|c|c|c|c|c|c|c|c|}
\hline $\begin{array}{l}\phi_{\gamma \gamma} \\
(\mathrm{deg})\end{array}$ & \multicolumn{2}{|c|}{$\begin{array}{c}\left\langle x_{B}\right\rangle=0.483 \\
\left\langle Q^{2}\right\rangle=2.707 \mathrm{GeV}^{2} \\
\left\langle t^{\prime}\right\rangle=-0.022 \mathrm{GeV}^{2}\end{array}$} & \multicolumn{2}{|c|}{$\begin{array}{c}\left\langle x_{B}\right\rangle=0.483 \\
\left\langle Q^{2}\right\rangle=2.708 \mathrm{GeV}^{2} \\
\left\langle t^{\prime}\right\rangle=-0.064 \mathrm{GeV}^{2}\end{array}$} & \multicolumn{2}{|c|}{$\begin{array}{c}\left\langle x_{B}\right\rangle=0.484 \\
\left\langle Q^{2}\right\rangle=2.713 \mathrm{GeV}^{2} \\
\left\langle t^{\prime}\right\rangle=-0.107 \mathrm{GeV}^{2}\end{array}$} & \multicolumn{2}{|c|}{$\begin{array}{c}\left\langle x_{B}\right\rangle=0.485 \\
\left\langle Q^{2}\right\rangle=2.715 \mathrm{GeV}^{2} \\
\left\langle t^{\prime}\right\rangle=-0.150 \mathrm{GeV}^{2}\end{array}$} & \multicolumn{2}{|c|}{$\begin{array}{c}\left\langle x_{B}\right\rangle=0.485 \\
\left\langle Q^{2}\right\rangle=2.717 \mathrm{GeV}^{2} \\
\left\langle t^{\prime}\right\rangle=-0.210 \mathrm{GeV}^{2}\end{array}$} \\
\hline 7.5 & $-0.91 \pm 2.66$ & $\begin{array}{c}+0.63 \\
-0.00\end{array}$ & $-43.80 \pm 2.99$ & $\begin{array}{l}+17.99 \\
-17.93\end{array}$ & $9.66 \pm 8.81$ & $\begin{array}{c}+1.44 \\
-0.00\end{array}$ & $-7.03 \pm 39.27$ & $\begin{array}{l}+6.78 \\
-0.00\end{array}$ & $24.30 \pm 33.48$ & $\begin{array}{c}+0.80 \\
-0.01\end{array}$ \\
\hline 22.5 & $1.17 \pm 3.03$ & $\begin{array}{c}+0.39 \\
-0.51 \\
\end{array}$ & $0.94 \pm 3.85$ & $\begin{array}{c}+1.76 \\
-0.00 \\
\end{array}$ & $-1.93 \pm 5.96$ & $\begin{array}{l}+1.69 \\
-0.51 \\
\end{array}$ & $-0.32 \pm 34.07$ & $\begin{array}{l}+5.55 \\
-0.00 \\
\end{array}$ & $-22.07 \pm 49.26$ & $\begin{array}{l}+0.00 \\
-1.51 \\
\end{array}$ \\
\hline 37.5 & $2.45 \pm 3.06$ & $\begin{array}{l}+0.00 \\
-0.64\end{array}$ & $2.83 \pm 3.51$ & $\begin{array}{l}+5.55 \\
-0.08\end{array}$ & $2.49 \pm 3.65$ & $\begin{array}{l}+0.39 \\
-0.51\end{array}$ & $-4.22 \pm 15.16$ & $\begin{array}{l}+0.19 \\
-3.99\end{array}$ & $-4.19 \pm 2.69$ & $\begin{array}{l}+0.19 \\
-2.69\end{array}$ \\
\hline 52.5 & $0.65 \pm 2.99$ & $\begin{array}{l}+0.00 \\
-1.18 \\
\end{array}$ & $3.33 \pm 2.71$ & $\begin{array}{l}+0.62 \\
-0.23\end{array}$ & $1.04 \pm 2.62$ & $\begin{array}{l}+0.00 \\
-1.06\end{array}$ & $2.53 \pm 5.84$ & $\begin{array}{l}+0.00 \\
-0.94 \\
\end{array}$ & $0.49 \pm 3.13$ & $\begin{array}{l}+0.54 \\
-0.00 \\
\end{array}$ \\
\hline 67.5 & $-0.06 \pm 2.96$ & $\begin{array}{c}+0.22 \\
-0.41 \\
\end{array}$ & $5.47 \pm 2.56$ & $\begin{array}{c}+0.44 \\
-0.00 \\
\end{array}$ & $1.13 \pm 2.22$ & $\begin{array}{l}+0.00 \\
-0.23 \\
\end{array}$ & $-2.16 \pm 2.53$ & $\begin{array}{l}+0.47 \\
-0.05 \\
\end{array}$ & $0.70 \pm 1.88$ & $\begin{array}{l}+0.05 \\
-0.11 \\
\end{array}$ \\
\hline 82.5 & $0.81 \pm 2.92$ & $\begin{array}{c}+1.22 \\
-0.00 \\
\end{array}$ & $3.18 \pm 2.31$ & $\begin{array}{c}+0.38 \\
-0.34 \\
\end{array}$ & $2.46 \pm 2.12$ & $\begin{array}{c}+0.00 \\
-0.69 \\
\end{array}$ & $3.01 \pm 2.50$ & $\begin{array}{l}+1.88 \\
-2.50 \\
\end{array}$ & $-1.75 \pm 1.44$ & $\begin{array}{c}+0.00 \\
-0.23 \\
\end{array}$ \\
\hline 97.5 & $1.88 \pm 2.50$ & $\begin{array}{l}+0.20 \\
-0.44 \\
\end{array}$ & $5.09 \pm 2.07$ & $\begin{array}{l}+0.30 \\
-0.89 \\
\end{array}$ & $0.95 \pm 1.81$ & $\begin{array}{l}+0.65 \\
-0.65 \\
\end{array}$ & $2.77 \pm 2.24$ & $\begin{array}{l}+0.06 \\
-0.57 \\
\end{array}$ & $-0.63 \pm 1.55$ & $\begin{array}{l}+0.14 \\
-0.15 \\
\end{array}$ \\
\hline 112.5 & $1.47 \pm 2.40$ & $\begin{array}{l}+0.00 \\
-0.25 \\
\end{array}$ & $1.02 \pm 1.99$ & $\begin{array}{l}+0.30 \\
-0.00 \\
\end{array}$ & $2.52 \pm 1.79$ & $\begin{array}{c}+0.41 \\
-0.00 \\
\end{array}$ & $2.17 \pm 1.89$ & $\begin{array}{l}+0.35 \\
-0.38 \\
\end{array}$ & $0.20 \pm 1.40$ & $\begin{array}{c}+0.12 \\
-0.39 \\
\end{array}$ \\
\hline 127.5 & $0.61 \pm 2.46$ & $\begin{array}{l}+0.39 \\
-0.45 \\
\end{array}$ & $2.05 \pm 1.89$ & $\begin{array}{l}+0.00 \\
-0.76 \\
\end{array}$ & $-0.13 \pm 1.70$ & $\begin{array}{c}+0.18 \\
-0.11 \\
\end{array}$ & $4.15 \pm 1.74$ & $\begin{array}{l}+0.51 \\
-0.26 \\
\end{array}$ & $0.14 \pm 1.38$ & $\begin{array}{c}+0.00 \\
-0.23 \\
\end{array}$ \\
\hline 142.5 & $0.46 \pm 2.32$ & $\begin{array}{c}+0.56 \\
-0.49 \\
\end{array}$ & $0.84 \pm 1.74$ & $\begin{array}{c}+0.15 \\
-0.11 \\
\end{array}$ & $0.75 \pm 1.82$ & $\begin{array}{c}+0.21 \\
-0.73 \\
\end{array}$ & $1.13 \pm 1.82$ & $\begin{array}{c}+0.15 \\
-0.46 \\
\end{array}$ & $3.50 \pm 1.50$ & $\begin{array}{c}+0.15 \\
-0.02 \\
\end{array}$ \\
\hline 157.5 & $-0.33 \pm 2.03$ & $\begin{array}{c}+0.00 \\
-0.25\end{array}$ & $1.42 \pm 1.80$ & $\begin{array}{c}+0.35 \\
-0.05\end{array}$ & $1.72 \pm 1.83$ & $\begin{array}{c}+0.12 \\
-0.46\end{array}$ & $0.86 \pm 1.97$ & $\begin{array}{c}+0.25 \\
-0.00\end{array}$ & $1.00 \pm 1.34$ & $\begin{array}{c}+0.10 \\
-0.22\end{array}$ \\
\hline 172.5 & $2.16 \pm 1.86$ & $\begin{array}{c}+0.27 \\
-0.01 \\
\end{array}$ & $-0.61 \pm 1.62$ & $\begin{array}{c}+0.22 \\
-0.17\end{array}$ & $0.09 \pm 1.57$ & $\begin{array}{c}+0.29 \\
-0.19\end{array}$ & $2.26 \pm 1.78$ & $\begin{array}{c}+0.24 \\
-0.09\end{array}$ & $1.36 \pm 1.43$ & $\begin{array}{c}+0.00 \\
-0.87 \\
\end{array}$ \\
\hline 187.5 & $-2.52 \pm 2.73$ & $\begin{array}{c}+1.51 \\
-0.00 \\
\end{array}$ & $1.55 \pm 1.92$ & $\begin{array}{c}+0.02 \\
-0.11 \\
\end{array}$ & $0.81 \pm 1.86$ & $\begin{array}{c}+0.43 \\
-0.57 \\
\end{array}$ & $1.08 \pm 1.59$ & $\begin{array}{c}+0.00 \\
-0.77 \\
\end{array}$ & $-1.33 \pm 1.52$ & $\begin{array}{c}+0.14 \\
-0.04 \\
\end{array}$ \\
\hline 202.5 & $0.20 \pm 2.26$ & $\begin{array}{c}+0.20 \\
-0.01\end{array}$ & $0.83 \pm 1.92$ & $\begin{array}{c}+0.29 \\
-0.58\end{array}$ & $0.23 \pm 1.75$ & $\begin{array}{c}+0.46 \\
-0.00\end{array}$ & $1.35 \pm 1.84$ & $\begin{array}{c}+0.00 \\
-0.31 \\
\end{array}$ & $0.27 \pm 1.14$ & $\begin{array}{c}+0.00 \\
-0.20\end{array}$ \\
\hline 217.5 & $-2.56 \pm 2.46$ & $\begin{array}{c}+0.53 \\
-0.24 \\
\end{array}$ & $-3.23 \pm 1.85$ & $\begin{array}{c}+0.16 \\
-0.39 \\
\end{array}$ & $1.96 \pm 1.66$ & $\begin{array}{c}+0.02 \\
-0.30 \\
\end{array}$ & $-2.97 \pm 1.58$ & $\begin{array}{c}+0.12 \\
-1.55 \\
\end{array}$ & $-1.25 \pm 1.01$ & $\begin{array}{c}+0.00 \\
-0.33 \\
\end{array}$ \\
\hline 232.5 & $-1.25 \pm 2.43$ & $\begin{array}{c}+0.67 \\
-0.93 \\
\end{array}$ & $-5.24 \pm 1.82$ & $\begin{array}{c}+0.22 \\
-0.21 \\
\end{array}$ & $-2.06 \pm 1.76$ & $\begin{array}{c}+0.53 \\
-0.29 \\
\end{array}$ & $-2.14 \pm 1.51$ & $\begin{array}{c}+0.00 \\
-0.52 \\
\end{array}$ & $-0.52 \pm 1.12$ & $\begin{array}{c}+0.01 \\
-0.05 \\
\end{array}$ \\
\hline 247.5 & $-1.32 \pm 2.56$ & $\begin{array}{c}+0.49 \\
-0.17 \\
\end{array}$ & $3.38 \pm 2.11$ & $\begin{array}{c}+0.80 \\
-0.00 \\
\end{array}$ & $-1.32 \pm 1.86$ & $\begin{array}{c}+0.13 \\
-0.36 \\
\end{array}$ & $0.10 \pm 1.59$ & $\begin{array}{l}+1.20 \\
-0.76 \\
\end{array}$ & $0.30 \pm 1.20$ & $\begin{array}{c}+0.28 \\
-0.20 \\
\end{array}$ \\
\hline 262.5 & $0.27 \pm 2.69$ & $\begin{array}{c}+0.85 \\
-0.00\end{array}$ & $-3.98 \pm 2.34$ & $\begin{array}{c}+0.71 \\
-0.58\end{array}$ & $-0.71 \pm 2.12$ & $\begin{array}{c}+0.74 \\
-0.34\end{array}$ & $-1.97 \pm 1.81$ & $\begin{array}{c}+1.00 \\
-0.00\end{array}$ & $-3.29 \pm 1.44$ & $\begin{array}{c}+0.38 \\
-0.01\end{array}$ \\
\hline 277.5 & $-0.67 \pm 2.81$ & $\begin{array}{c}+0.04 \\
-0.41\end{array}$ & $-2.77 \pm 2.85$ & $\begin{array}{c}+0.04 \\
-0.91\end{array}$ & $-3.12 \pm 2.57$ & $\begin{array}{c}+0.22 \\
-1.24\end{array}$ & $-4.12 \pm 2.17$ & $\begin{array}{c}+0.35 \\
-0.00\end{array}$ & $-3.35 \pm 1.60$ & $\begin{array}{c}+0.26 \\
-0.03\end{array}$ \\
\hline 292.5 & $-1.73 \pm 3.14$ & $\begin{array}{c}+2.08 \\
-0.00 \\
\end{array}$ & $-0.78 \pm 2.95$ & $\begin{array}{c}+0.00 \\
-0.77\end{array}$ & $0.99 \pm 2.96$ & $\begin{array}{c}+0.18 \\
-0.76\end{array}$ & $-2.71 \pm 2.39$ & $\begin{array}{c}+0.19 \\
-0.41 \\
\end{array}$ & $21.23 \pm 2.61$ & $\begin{array}{c}+25.85 \\
-0.00\end{array}$ \\
\hline 307.5 & $-7.78 \pm 3.23$ & $\begin{array}{c}+0.46 \\
-0.10 \\
\end{array}$ & $3.56 \pm 3.44$ & $\begin{array}{c}+0.67 \\
-0.00 \\
\end{array}$ & $-0.11 \pm 3.28$ & $\begin{array}{c}+0.31 \\
-0.00 \\
\end{array}$ & $-1.96 \pm 3.08$ & $\begin{array}{c}+0.41 \\
-0.00 \\
\end{array}$ & $-5.78 \pm 3.53$ & $\begin{array}{c}+0.09 \\
-0.29 \\
\end{array}$ \\
\hline 322.5 & $3.59 \pm 3.35$ & $\begin{array}{c}+0.86 \\
-0.68\end{array}$ & $-5.33 \pm 3.96$ & $\begin{array}{c}+9.32 \\
-1.24 \\
\end{array}$ & $0.72 \pm 4.20$ & $\begin{array}{c}+1.96 \\
-0.45\end{array}$ & $-0.10 \pm 2.79$ & $\begin{array}{c}+0.61 \\
-1.20 \\
\end{array}$ & $7.20 \pm 14.89$ & $\begin{array}{r}+3.40 \\
-14.89 \\
\end{array}$ \\
\hline 337.5 & $-1.94 \pm 3.40$ & $\begin{array}{c}+0.04 \\
-0.04 \\
\end{array}$ & $-3.44 \pm 3.75$ & $\begin{array}{c}+14.52 \\
-0.00 \\
\end{array}$ & $-7.30 \pm 6.29$ & $\begin{array}{c}+1.65 \\
-0.75 \\
\end{array}$ & $23.35 \pm 7.59$ & $\begin{array}{c}+2.67 \\
-0.90 \\
\end{array}$ & $-41.59 \pm 47.25$ & $\begin{array}{c}+15.09 \\
-2.20 \\
\end{array}$ \\
\hline 352.5 & $-4.24 \pm 3.82$ & $\begin{array}{c}+0.78 \\
-0.33 \\
\end{array}$ & $-3.46 \pm 2.77$ & $\begin{array}{c}+1.37 \\
-0.85 \\
\end{array}$ & $-3.77 \pm 9.08$ & $\begin{array}{c}+1.40 \\
-3.13 \\
\end{array}$ & $14.88 \pm 19.54$ & $\begin{array}{c}+0.00 \\
-8.97 \\
\end{array}$ & $16.42 \pm 22.63$ & $\begin{array}{c}+0.64 \\
-0.79 \\
\end{array}$ \\
\hline
\end{tabular}

Table E.1: Table of helicity-dependent extracted cross section values (pb/GeV4) for Kin481. The \pm values are the statistical errors, and the separated + and - are the systematic error introduced from the missing mass cut. The overall systematic uncertainties from table 6.4 are not represented here. 


\begin{tabular}{|c|c|c|c|c|c|c|c|c|c|c|}
\hline $\begin{array}{l}\phi_{\gamma \gamma} \\
\text { (deg) }\end{array}$ & \multicolumn{2}{|c|}{$\begin{array}{c}\left\langle x_{B}\right\rangle=0.497 \\
\left\langle Q^{2}\right\rangle=4.497 \mathrm{GeV}^{2} \\
\left\langle t^{\prime}\right\rangle=-0.031 \mathrm{GeV}^{2}\end{array}$} & \multicolumn{2}{|c|}{$\begin{array}{c}\left\langle x_{B}\right\rangle=0.501 \\
\left\langle Q^{2}\right\rangle=4.528 \mathrm{GeV}^{2} \\
\left\langle t^{\prime}\right\rangle=-0.093 \mathrm{GeV}^{2}\end{array}$} & \multicolumn{2}{|c|}{$\begin{array}{c}\left\langle x_{B}\right\rangle=0.504 \\
\left\langle Q^{2}\right\rangle=4.558 \mathrm{GeV}^{2} \\
\left\langle t^{\prime}\right\rangle=-0.154 \mathrm{GeV}^{2}\end{array}$} & \multicolumn{2}{|c|}{$\begin{array}{c}\left\langle x_{B}\right\rangle=0.506 \\
\left\langle Q^{2}\right\rangle=4.573 \mathrm{GeV}^{2} \\
\left\langle t^{\prime}\right\rangle=-0.216 \mathrm{GeV}^{2}\end{array}$} & \multicolumn{2}{|c|}{$\begin{array}{c}\left\langle x_{B}\right\rangle=0.508 \\
\left\langle Q^{2}\right\rangle=4.593 \mathrm{GeV}^{2} \\
\left\langle t^{\prime}\right\rangle=-0.305 \mathrm{GeV}^{2}\end{array}$} \\
\hline 7.5 & $0.32 \pm 0.62$ & $\begin{array}{c}+0.34 \\
-0.00\end{array}$ & $0.66 \pm 0.54$ & $\begin{array}{c}+0.11 \\
-0.05\end{array}$ & $0.16 \pm 0.95$ & $\begin{array}{c}+0.36 \\
-0.00\end{array}$ & $0.93 \pm 1.29$ & $\begin{array}{c}+0.08 \\
-0.08\end{array}$ & $2.47 \pm 3.45$ & $\begin{array}{c}+1.22 \\
-0.00\end{array}$ \\
\hline 22.5 & $0.75 \pm 0.61$ & $\begin{array}{l}+0.07 \\
-0.20\end{array}$ & $0.64 \pm 0.45$ & $\begin{array}{l}+0.12 \\
-0.12\end{array}$ & $0.95 \pm 0.71$ & $\begin{array}{l}+0.13 \\
-0.04\end{array}$ & $-0.62 \pm 1.02$ & $\begin{array}{l}+0.01 \\
-0.26\end{array}$ & $0.65 \pm 1.57$ & $\begin{array}{c}+0.03 \\
-0.04\end{array}$ \\
\hline 37.5 & $-0.09 \pm 0.51$ & $\begin{array}{l}+0.10 \\
-0.05\end{array}$ & $0.30 \pm 0.43$ & $\begin{array}{l}+0.16 \\
-0.05\end{array}$ & $0.53 \pm 0.44$ & $\begin{array}{l}+0.07 \\
-0.02\end{array}$ & $0.27 \pm 0.62$ & $\begin{array}{l}+0.00 \\
-0.16\end{array}$ & $0.03 \pm 0.53$ & $\begin{array}{l}+0.02 \\
-0.11\end{array}$ \\
\hline 52.5 & $-0.03 \pm 0.49$ & $\begin{array}{l}+0.15 \\
-0.00\end{array}$ & $0.52 \pm 0.33$ & $\begin{array}{l}+0.12 \\
-0.02\end{array}$ & $0.72 \pm 0.33$ & $\begin{array}{l}+0.07 \\
-0.01\end{array}$ & $0.42 \pm 0.41$ & $\begin{array}{l}+0.02 \\
-0.06\end{array}$ & $0.31 \pm 0.41$ & $\begin{array}{l}+0.00 \\
-0.27\end{array}$ \\
\hline 67.5 & $0.37 \pm 0.47$ & $\begin{array}{l}+0.23 \\
-0.02\end{array}$ & $0.72 \pm 0.31$ & $\begin{array}{l}+0.05 \\
-0.02\end{array}$ & $0.43 \pm 0.28$ & $\begin{array}{l}+0.04 \\
-0.00\end{array}$ & $0.54 \pm 0.31$ & $\begin{array}{l}+0.12 \\
-0.00\end{array}$ & $0.67 \pm 0.26$ & $\begin{array}{l}+0.05 \\
-0.07\end{array}$ \\
\hline 82.5 & $0.77 \pm 0.45$ & $\begin{array}{l}+0.11 \\
-0.30\end{array}$ & $0.35 \pm 0.29$ & $\begin{array}{l}+0.00 \\
-0.06\end{array}$ & $0.47 \pm 0.28$ & $\begin{array}{l}+0.01 \\
-0.03\end{array}$ & $0.24 \pm 0.28$ & $\begin{array}{l}+0.06 \\
-0.09\end{array}$ & $0.50 \pm 0.22$ & $\begin{array}{l}+0.09 \\
-0.02\end{array}$ \\
\hline 97.5 & $0.76 \pm 0.43$ & $\begin{array}{c}+0.00 \\
-0.10\end{array}$ & $0.51 \pm 0.27$ & $\begin{array}{l}+0.09 \\
-0.00\end{array}$ & $0.21 \pm 0.24$ & $\begin{array}{l}+0.00 \\
-0.06\end{array}$ & $0.46 \pm 0.23$ & $\begin{array}{l}+0.01 \\
-0.05\end{array}$ & $0.11 \pm 0.20$ & $\begin{array}{c}+0.04 \\
-0.03\end{array}$ \\
\hline 112.5 & $0.32 \pm 0.39$ & $\begin{array}{l}+0.00 \\
-0.16\end{array}$ & $0.78 \pm 0.29$ & $\begin{array}{l}+0.01 \\
-0.02\end{array}$ & $0.58 \pm 0.24$ & $\begin{array}{l}+0.06 \\
-0.02\end{array}$ & $0.66 \pm 0.23$ & $\begin{array}{l}+0.05 \\
-0.02\end{array}$ & $0.23 \pm 0.17$ & $\begin{array}{l}+0.06 \\
-0.02\end{array}$ \\
\hline 127.5 & $0.02 \pm 0.38$ & $\begin{array}{l}+0.00 \\
-0.25\end{array}$ & $0.46 \pm 0.26$ & $\begin{array}{l}+0.10 \\
-0.05\end{array}$ & $0.34 \pm 0.23$ & $\begin{array}{l}+0.07 \\
-0.00\end{array}$ & $0.61 \pm 0.20$ & $\begin{array}{l}+0.08 \\
-0.04\end{array}$ & $0.26 \pm 0.15$ & $\begin{array}{l}+0.00 \\
-0.15\end{array}$ \\
\hline 142.5 & $0.05 \pm 0.34$ & $\begin{array}{l}+0.10 \\
-0.23\end{array}$ & $0.60 \pm 0.25$ & $\begin{array}{l}+0.06 \\
-0.02\end{array}$ & $0.21 \pm 0.22$ & $\begin{array}{l}+0.03 \\
-0.00\end{array}$ & $0.19 \pm 0.22$ & $\begin{array}{l}+0.07 \\
-0.04\end{array}$ & $0.29 \pm 0.14$ & $\begin{array}{l}+0.00 \\
-0.14\end{array}$ \\
\hline 157.5 & $0.05 \pm 0.33$ & $\begin{array}{l}+0.00 \\
-0.05\end{array}$ & $0.44 \pm 0.22$ & $\begin{array}{l}+0.00 \\
-0.12\end{array}$ & $0.52 \pm 0.22$ & $\begin{array}{l}+0.00 \\
-0.15\end{array}$ & $0.06 \pm 0.22$ & $\begin{array}{l}+0.03 \\
-0.03\end{array}$ & $0.16 \pm 0.16$ & $\begin{array}{l}+0.03 \\
-0.06\end{array}$ \\
\hline 172.5 & $-0.08 \pm 0.32$ & $\begin{array}{l}+0.12 \\
-0.00\end{array}$ & $0.04 \pm 0.22$ & $\begin{array}{l}+0.00 \\
-0.08\end{array}$ & $0.19 \pm 0.22$ & $\begin{array}{l}+0.18 \\
0.06-\end{array}$ & $-0.05 \pm 0.23$ & $\begin{array}{l}+0.06 \\
-0.04\end{array}$ & $0.14 \pm 0.17$ & $\begin{array}{c}+0.02 \\
-0.00\end{array}$ \\
\hline 187.5 & $-0.07 \pm 0.27$ & $\begin{array}{l}+0.02 \\
-0.05\end{array}$ & $0.28 \pm 0.21$ & $\begin{array}{l}+0.00 \\
-0.05\end{array}$ & $0.49 \pm 0.23$ & $\begin{array}{l}+0.00 \\
-0.05\end{array}$ & $0.05 \pm 0.23$ & $\begin{array}{l}+0.04 \\
-0.00\end{array}$ & $-0.16 \pm 0.16$ & $\begin{array}{l}+0.02 \\
-0.04\end{array}$ \\
\hline 202.5 & $0.09 \pm 0.33$ & $\begin{array}{l}+0.08 \\
-0.08\end{array}$ & $-0.16 \pm 0.24$ & $\begin{array}{l}+0.07 \\
-0.01\end{array}$ & $0.04 \pm 0.20$ & $\begin{array}{l}+0.02 \\
-0.00\end{array}$ & $-0.01 \pm 0.21$ & $\begin{array}{l}+0.00 \\
-0.09\end{array}$ & $-0.15 \pm 0.15$ & $\begin{array}{l}+0.03 \\
-0.00\end{array}$ \\
\hline 217.5 & $0.01 \pm 0.31$ & $\begin{array}{l}+0.13 \\
-0.00\end{array}$ & $-0.31 \pm 0.23$ & $\begin{array}{l}+0.11 \\
-0.00\end{array}$ & $0.07 \pm 0.20$ & $\begin{array}{l}+0.00 \\
-0.07\end{array}$ & $-0.27 \pm 0.22$ & $\begin{array}{l}+0.00 \\
-0.08\end{array}$ & $-0.30 \pm 0.15$ & $\begin{array}{l}+0.01 \\
-0.10\end{array}$ \\
\hline 232.5 & $-0.02 \pm 0.35$ & $\begin{array}{l}+0.18 \\
-0.00\end{array}$ & $-0.29 \pm 0.26$ & $\begin{array}{l}+0.04 \\
-0.10\end{array}$ & $-0.18 \pm 0.23$ & $\begin{array}{l}+0.04 \\
-0.00\end{array}$ & $-0.49 \pm 0.22$ & $\begin{array}{l}+0.03 \\
-0.04\end{array}$ & $-0.28 \pm 0.14$ & $\begin{array}{l}+0.02 \\
-0.02\end{array}$ \\
\hline 247.5 & $-0.75 \pm 0.39$ & $\begin{array}{c}+0.08 \\
-0.04\end{array}$ & $0.28 \pm 0.29$ & $\begin{array}{l}+0.00 \\
-0.03\end{array}$ & $-0.35 \pm 0.25$ & $\begin{array}{l}+0.00 \\
-0.06\end{array}$ & $-0.21 \pm 0.23$ & $\begin{array}{l}+0.00 \\
-0.03\end{array}$ & $-0.42 \pm 0.16$ & $\begin{array}{l}+0.02 \\
-0.00\end{array}$ \\
\hline 262.5 & $-1.00 \pm 0.44$ & $\begin{array}{l}+0.06 \\
-0.00\end{array}$ & $-0.08 \pm 0.32$ & $\begin{array}{l}+0.00 \\
-0.16\end{array}$ & $-0.39 \pm 0.27$ & $\begin{array}{l}+0.05 \\
-0.00\end{array}$ & $-0.51 \pm 0.24$ & $\begin{array}{l}+0.00 \\
-0.06\end{array}$ & $-0.25 \pm 0.17$ & $\begin{array}{l}+0.01 \\
-0.06\end{array}$ \\
\hline 277.5 & $-1.09 \pm 0.45$ & $\begin{array}{l}+0.02 \\
-0.03\end{array}$ & $-0.19 \pm 0.32$ & $\begin{array}{l}+0.00 \\
-0.10\end{array}$ & $-0.70 \pm 0.27$ & $\begin{array}{l}+0.04 \\
-0.03\end{array}$ & $-0.61 \pm 0.26$ & $\begin{array}{l}+0.00 \\
-0.08\end{array}$ & $-0.32 \pm 0.20$ & $\begin{array}{c}+0.04 \\
-0.03\end{array}$ \\
\hline 292.5 & $-0.75 \pm 0.47$ & $\begin{array}{l}+0.09 \\
-0.05\end{array}$ & $-0.60 \pm 0.34$ & $\begin{array}{l}+0.01 \\
-0.05\end{array}$ & $-1.19 \pm 0.30$ & $\begin{array}{l}+0.10 \\
-0.00\end{array}$ & $-0.26 \pm 0.26$ & $\begin{array}{l}+0.01 \\
-0.04\end{array}$ & $-0.23 \pm 0.24$ & $\begin{array}{l}+0.03 \\
-0.03\end{array}$ \\
\hline 307.5 & $-1.08 \pm 0.46$ & $\begin{array}{l}+0.05 \\
-0.02\end{array}$ & $-0.15 \pm 0.33$ & $\begin{array}{l}+0.00 \\
-0.02\end{array}$ & $-0.13 \pm 0.34$ & $\begin{array}{l}+0.00 \\
-0.11\end{array}$ & $-1.18 \pm 0.39$ & $\begin{array}{l}+0.13 \\
-0.00\end{array}$ & $-0.38 \pm 0.36$ & $\begin{array}{l}+0.03 \\
-0.09\end{array}$ \\
\hline 322.5 & $-0.61 \pm 0.49$ & $\begin{array}{l}+0.11 \\
-0.05\end{array}$ & $0.09 \pm 0.40$ & $\begin{array}{l}+0.05 \\
-0.07\end{array}$ & $-0.50 \pm 0.46$ & $\begin{array}{l}+0.00 \\
-0.09\end{array}$ & $-1.35 \pm 0.62$ & $\begin{array}{l}+0.17 \\
-0.16\end{array}$ & $-1.42 \pm 0.87$ & $\begin{array}{l}+0.08 \\
-0.05\end{array}$ \\
\hline 337.5 & $0.26 \pm 0.53$ & $\begin{array}{l}+0.04 \\
-0.13\end{array}$ & $-0.46 \pm 0.50$ & $\begin{array}{l}+0.11 \\
-0.12\end{array}$ & $-0.92 \pm 0.61$ & $\begin{array}{l}+0.06 \\
-0.25\end{array}$ & $-1.17 \pm 1.13$ & $\begin{array}{l}+0.03 \\
-0.48\end{array}$ & $-0.87 \pm 1.00$ & $\begin{array}{l}+0.03 \\
-0.06\end{array}$ \\
\hline 352.5 & $0.16 \pm 0.50$ & $\begin{array}{l}+0.09 \\
-0.00\end{array}$ & $-0.48 \pm 0.50$ & $\begin{array}{l}+0.00 \\
-0.13\end{array}$ & $-0.34 \pm 0.85$ & $\begin{array}{l}+0.07 \\
-0.09\end{array}$ & $1.82 \pm 1.28$ & $\begin{array}{l}+0.12 \\
-0.14\end{array}$ & $-1.01 \pm 2.45$ & $\begin{array}{l}+0.01 \\
-0.11\end{array}$ \\
\hline
\end{tabular}

Table E.2: Table of helicity-dependent extracted cross section values (pb/GeV ${ }^{4}$ ) for Kin482. The \pm values are the statistical errors, and the separated + and - are the systematic error introduced from the missing mass cut. The overall systematic uncertainties from table 6.4 are not represented here. 


\begin{tabular}{|c|c|c|c|c|c|c|c|c|c|c|}
\hline \multirow{2}{*}{$\begin{array}{c}\phi_{\gamma \gamma} \\
\text { (deg) }\end{array}$} & \multicolumn{2}{|c|}{$\begin{array}{c}\left\langle x_{B}\right\rangle=0.483 \\
\left\langle Q^{2}\right\rangle=5.331 \mathrm{GeV}^{2} \\
\left\langle t^{\prime}\right\rangle=-0.030 \mathrm{GeV}^{2}\end{array}$} & \multicolumn{2}{|c|}{$\begin{array}{c}\left\langle x_{B}\right\rangle=0.483 \\
\left\langle Q^{2}\right\rangle=5.339 \mathrm{GeV}^{2} \\
\left\langle t^{\prime}\right\rangle=-0.088 \mathrm{GeV}^{2}\end{array}$} & \multicolumn{2}{|c|}{$\begin{array}{c}\left\langle x_{B}\right\rangle=0.485 \\
\left\langle Q^{2}\right\rangle=5.360 \mathrm{GeV}^{2} \\
\left\langle t^{\prime}\right\rangle=-0.147 \mathrm{GeV}^{2}\end{array}$} & \multicolumn{2}{|c|}{$\begin{array}{c}\left\langle x_{B}\right\rangle=0.486 \\
\left\langle Q^{2}\right\rangle=5.371 \mathrm{GeV}^{2} \\
\left\langle t^{\prime}\right\rangle=-0.206 \mathrm{GeV}^{2}\end{array}$} & \multicolumn{2}{|c|}{$\begin{array}{c}\left\langle x_{B}\right\rangle=0.486 \\
\left\langle Q^{2}\right\rangle=5.379 \mathrm{GeV}^{2} \\
\left\langle t^{\prime}\right\rangle=-0.291 \mathrm{GeV}^{2}\end{array}$} \\
\hline & $-0.25 \pm 0.41$ & $\begin{array}{c}+0.03 \\
-0.12\end{array}$ & $0.53 \pm 0.39$ & $\begin{array}{c}+0.00 \\
-0.08\end{array}$ & $-0.36 \pm 0.55$ & $\begin{array}{c}+0.00 \\
-0.30\end{array}$ & $0.52 \pm 0.99$ & $\begin{array}{c}+0.00 \\
-0.19\end{array}$ & $0.57 \pm 1.93$ & $\begin{array}{c}+0.02 \\
-0.05\end{array}$ \\
\hline 22.5 & $0.38 \pm 0.37$ & $\begin{array}{l}+0.06 \\
-0.03\end{array}$ & $0.42 \pm 0.36$ & $\begin{array}{l}+0.04 \\
-0.00\end{array}$ & $0.58 \pm 0.42$ & $\begin{array}{l}+0.14 \\
-0.02\end{array}$ & $0.85 \pm 0.55$ & $\begin{array}{l}+0.03 \\
-0.15\end{array}$ & $0.59 \pm 1.03$ & $\begin{array}{l}+0.02 \\
-0.07\end{array}$ \\
\hline 37.5 & $0.66 \pm 0.35$ & $\begin{array}{l}+0.00 \\
-0.35\end{array}$ & $0.85 \pm 0.31$ & $\begin{array}{l}+0.11 \\
-0.00\end{array}$ & $0.57 \pm 0.33$ & $\begin{array}{l}+0.03 \\
-0.12\end{array}$ & $0.19 \pm 0.40$ & $\begin{array}{l}+0.14 \\
-0.01\end{array}$ & $0.98 \pm 0.39$ & $\begin{array}{l}+0.13 \\
-0.39\end{array}$ \\
\hline 52.5 & $0.57 \pm 0.38$ & $\begin{array}{l}+0.23 \\
-0.07\end{array}$ & $0.12 \pm 0.30$ & $\begin{array}{l}+0.01 \\
-0.01\end{array}$ & $0.90 \pm 0.27$ & $\begin{array}{l}+0.05 \\
-0.22\end{array}$ & $0.56 \pm 0.30$ & $\begin{array}{l}+0.04 \\
-0.00\end{array}$ & $0.26 \pm 0.26$ & $\begin{array}{l}+0.04 \\
-0.02\end{array}$ \\
\hline 67.5 & $0.24 \pm 0.34$ & $\begin{array}{l}+0.09 \\
-0.00\end{array}$ & $0.35 \pm 0.27$ & $\begin{array}{l}+0.14 \\
-0.19\end{array}$ & $0.93 \pm 0.25$ & $\begin{array}{l}+0.04 \\
-0.06\end{array}$ & $0.75 \pm 0.24$ & $\begin{array}{l}+0.10 \\
-0.09\end{array}$ & $0.57 \pm 0.19$ & $\begin{array}{l}+0.00 \\
-0.04\end{array}$ \\
\hline 82.5 & $0.64 \pm 0.32$ & $\begin{array}{l}+0.01 \\
-0.14\end{array}$ & $0.36 \pm 0.27$ & $\begin{array}{l}+0.15 \\
-0.02\end{array}$ & $0.35 \pm 0.21$ & $\begin{array}{l}+0.01 \\
-0.04\end{array}$ & $0.22 \pm 0.22$ & $\begin{array}{l}+0.01 \\
-0.06\end{array}$ & $0.39 \pm 0.17$ & $\begin{array}{l}+0.00 \\
-0.06\end{array}$ \\
\hline 97.5 & $0.34 \pm 0.31$ & $\begin{array}{l}+0.06 \\
-0.00\end{array}$ & $0.09 \pm 0.24$ & $\begin{array}{l}+0.01 \\
-0.08\end{array}$ & $0.14 \pm 0.21$ & $\begin{array}{l}+0.04 \\
-0.00\end{array}$ & $0.38 \pm 0.19$ & $\begin{array}{c}+0.03 \\
-0.02\end{array}$ & $0.29 \pm 0.14$ & $\begin{array}{l}+0.02 \\
-0.00\end{array}$ \\
\hline 112.5 & $0.06 \pm 0.32$ & $\begin{array}{l}+0.14 \\
-0.03\end{array}$ & $0.89 \pm 0.20$ & $\begin{array}{l}+0.01 \\
-0.03\end{array}$ & $0.29 \pm 0.18$ & $\begin{array}{l}+0.05 \\
-0.02\end{array}$ & $0.20 \pm 0.17$ & $\begin{array}{l}+0.02 \\
-0.06\end{array}$ & $0.12 \pm 0.13$ & $\begin{array}{c}+0.001 \\
-0.00\end{array}$ \\
\hline 127.5 & $0.59 \pm 0.28$ & $\begin{array}{l}+0.06 \\
-0.01\end{array}$ & $0.17 \pm 0.21$ & $\begin{array}{l}+0.06 \\
-0.00\end{array}$ & $-0.03 \pm 0.16$ & $\begin{array}{l}+0.03 \\
-0.00\end{array}$ & $0.38 \pm 0.19$ & $\begin{array}{l}+0.13 \\
-0.00\end{array}$ & $0.19 \pm 0.11$ & $\begin{array}{l}+0.00 \\
-0.05\end{array}$ \\
\hline 142.5 & $0.08 \pm 0.26$ & $\begin{array}{l}+0.00 \\
-0.13\end{array}$ & $0.29 \pm 0.20$ & $\begin{array}{l}+0.00 \\
-0.03\end{array}$ & $0.08 \pm 0.17$ & $\begin{array}{l}+0.03 \\
-0.00\end{array}$ & $0.15 \pm 0.17$ & $\begin{array}{l}+0.00 \\
-0.07\end{array}$ & $0.17 \pm 0.10$ & $\begin{array}{l}+0.02 \\
-0.00\end{array}$ \\
\hline 157.5 & $0.08 \pm 0.27$ & $\begin{array}{l}+0.03 \\
-0.02\end{array}$ & $0.47 \pm 0.18$ & $\begin{array}{l}+0.05 \\
-0.01\end{array}$ & $0.10 \pm 0.18$ & $\begin{array}{c}+0.00 \\
-0.04\end{array}$ & $0.08 \pm 0.17$ & $\begin{array}{l}+0.06 \\
-0.16\end{array}$ & $-0.04 \pm 0.11$ & $\begin{array}{l}+0.02 \\
-0.00\end{array}$ \\
\hline 172.5 & $-0.28 \pm 0.26$ & $\begin{array}{l}+0.23 \\
-0.21\end{array}$ & $-0.13 \pm 0.19$ & $\begin{array}{l}+0.03 \\
-0.04\end{array}$ & $0.22 \pm 0.17$ & $\begin{array}{l}+0.04 \\
-0.04\end{array}$ & $-0.03 \pm 0.17$ & $\begin{array}{l}+0.02 \\
-0.00\end{array}$ & $0.06 \pm 0.12$ & $\begin{array}{l}+0.00 \\
-0.02\end{array}$ \\
\hline 187.5 & $0.26 \pm 0.23$ & $\begin{array}{l}+0.01 \\
-0.01\end{array}$ & $-0.02 \pm 0.18$ & $\begin{array}{l}+0.03 \\
-0.01\end{array}$ & $-0.25 \pm 0.17$ & $\begin{array}{l}+0.02 \\
-0.05\end{array}$ & $0.10 \pm 0.16$ & $\begin{array}{l}+0.13 \\
-0.00\end{array}$ & $-0.05 \pm 0.12$ & $\begin{array}{l}+0.01 \\
-0.03\end{array}$ \\
\hline 202.5 & $-0.42 \pm 0.26$ & $\begin{array}{c}+0.11 \\
-0.00\end{array}$ & $-0.41 \pm 0.19$ & $\begin{array}{l}+0.03 \\
-0.02\end{array}$ & $-0.10 \pm 0.16$ & $\begin{array}{l}+0.02 \\
-0.02\end{array}$ & $-0.27 \pm 0.16$ & $\begin{array}{l}+0.04 \\
-0.00\end{array}$ & $-0.09 \pm 0.12$ & $\begin{array}{l}+0.01 \\
-0.07\end{array}$ \\
\hline 217.5 & $-0.05 \pm 0.25$ & $\begin{array}{l}+0.14 \\
-0.20\end{array}$ & $-0.38 \pm 0.18$ & $\begin{array}{l}+0.04 \\
-0.00\end{array}$ & $-0.22 \pm 0.16$ & $\begin{array}{l}+0.00 \\
-0.02\end{array}$ & $-0.29 \pm 0.16$ & $\begin{array}{l}+0.02 \\
-0.03\end{array}$ & $-0.01 \pm 0.10$ & $\begin{array}{c}+0.01 \\
-0.03\end{array}$ \\
\hline 232.5 & $-0.22 \pm 0.28$ & $\begin{array}{c}+0.00 \\
-0.10\end{array}$ & $-0.13 \pm 0.21$ & $\begin{array}{l}+0.02 \\
-0.00\end{array}$ & $-0.43 \pm 0.17$ & $\begin{array}{l}+0.09 \\
-0.01\end{array}$ & $-0.23 \pm 0.17$ & $\begin{array}{l}+0.04 \\
-0.04\end{array}$ & $-0.02 \pm 0.11$ & $\begin{array}{l}+0.01 \\
-0.02\end{array}$ \\
\hline 247.5 & $-0.70 \pm 0.29$ & $\begin{array}{c}+0.09 \\
-0.03\end{array}$ & $-0.54 \pm 0.24$ & $\begin{array}{l}+0.07 \\
-0.00\end{array}$ & $-0.47 \pm 0.18$ & $\begin{array}{l}+0.01 \\
-0.04\end{array}$ & $-0.37 \pm 0.17$ & $\begin{array}{l}+0.10 \\
-0.00\end{array}$ & $-0.17 \pm 0.12$ & $\begin{array}{c}+0.01 \\
-0.04\end{array}$ \\
\hline 262.5 & $-0.34 \pm 0.33$ & $\begin{array}{l}+0.16 \\
-0.00\end{array}$ & $-0.07 \pm 0.25$ & $\begin{array}{l}+0.04 \\
-0.00\end{array}$ & $-0.30 \pm 0.22$ & $\begin{array}{l}+0.00 \\
-0.03\end{array}$ & $-0.56 \pm 0.20$ & $\begin{array}{l}+0.00 \\
-0.07\end{array}$ & $-0.20 \pm 0.14$ & $\begin{array}{l}+0.03 \\
-0.03\end{array}$ \\
\hline 277.5 & $0.03 \pm 0.34$ & $\begin{array}{l}+0.08 \\
-0.09\end{array}$ & $-0.27 \pm 0.27$ & $\begin{array}{l}+0.00 \\
-0.14\end{array}$ & $-0.39 \pm 0.24$ & $\begin{array}{l}+0.04 \\
-0.01\end{array}$ & $-0.43 \pm 0.21$ & $\begin{array}{l}+0.04 \\
-0.01\end{array}$ & $-0.48 \pm 0.15$ & $\begin{array}{l}+0.00 \\
-0.07\end{array}$ \\
\hline 292.5 & $-0.71 \pm 0.34$ & $\begin{array}{l}+0.00 \\
-0.05\end{array}$ & $-0.24 \pm 0.28$ & $\begin{array}{l}+0.05 \\
-0.02\end{array}$ & $-0.63 \pm 0.25$ & $\begin{array}{l}+0.06 \\
-0.10\end{array}$ & $-0.17 \pm 0.25$ & $\begin{array}{l}+0.04 \\
-0.13\end{array}$ & $-0.44 \pm 0.19$ & $\begin{array}{l}+0.01 \\
-0.09\end{array}$ \\
\hline 307.5 & $-0.13 \pm 0.36$ & $\begin{array}{c}+0.04 \\
-0.09\end{array}$ & $0.37 \pm 0.32$ & $\begin{array}{l}+0.03 \\
-0.03\end{array}$ & $-1.03 \pm 0.26$ & $\begin{array}{l}+0.00 \\
-0.08\end{array}$ & $-0.37 \pm 0.29$ & $\begin{array}{l}+0.00 \\
-0.10\end{array}$ & $-0.24 \pm 0.24$ & $\begin{array}{c}+0.02 \\
-0.03\end{array}$ \\
\hline 322.5 & $0.30 \pm 0.39$ & $\begin{array}{l}+0.08 \\
-0.39\end{array}$ & $-0.66 \pm 0.33$ & $\begin{array}{l}+0.11 \\
-0.00\end{array}$ & $-0.70 \pm 0.35$ & $\begin{array}{l}+0.02 \\
-0.10\end{array}$ & $-0.72 \pm 0.39$ & $\begin{array}{c}+0.00 \\
-0.42\end{array}$ & $0.26 \pm 0.50$ & $\begin{array}{l}+0.12 \\
-0.01\end{array}$ \\
\hline 337.5 & $-0.04 \pm 0.38$ & $\begin{array}{l}+0.03 \\
-0.00\end{array}$ & $-0.06 \pm 0.35$ & $\begin{array}{l}+2.30 \\
-0.08\end{array}$ & $0.11 \pm 0.43$ & $\begin{array}{l}+0.37 \\
-0.07\end{array}$ & $-0.05 \pm 0.76$ & $\begin{array}{l}+0.07 \\
-0.02\end{array}$ & $0.20 \pm 1.06$ & $\begin{array}{l}+0.13 \\
-0.00\end{array}$ \\
\hline 352.5 & $-0.01 \pm 0.37$ & $\begin{array}{l}+0.00 \\
-0.01\end{array}$ & $-0.12 \pm 0.39$ & $\begin{array}{l}+0.03 \\
-0.05\end{array}$ & $-0.79 \pm 0.57$ & $\begin{array}{l}+0.00 \\
-0.17\end{array}$ & $-2.35 \pm 1.08$ & $\begin{array}{l}+0.01 \\
-0.01\end{array}$ & $0.52 \pm 0.72$ & $\begin{array}{l}+0.03 \\
-0.07\end{array}$ \\
\hline
\end{tabular}

Table E.3: Table of helicity-dependent extracted cross section values (pb/GeV ${ }^{4}$ ) for Kin483. The \pm values are the statistical errors, and the separated + and - are the systematic error introduced from the missing mass cut. The overall systematic uncertainties from table 6.4 are not represented here. 


\begin{tabular}{|c|c|c|c|c|c|c|c|c|c|c|}
\hline $\begin{array}{l}\phi_{\gamma \gamma} \\
(\mathrm{deg})\end{array}$ & \multicolumn{2}{|c|}{$\begin{array}{c}\left\langle x_{B}\right\rangle=0.494 \\
\left\langle Q^{2}\right\rangle=7.044 \mathrm{GeV}^{2} \\
\left\langle t^{\prime}\right\rangle=-0.043 \mathrm{GeV}^{2}\end{array}$} & \multicolumn{2}{|c|}{$\begin{array}{c}\left\langle x_{B}\right\rangle=0.498 \\
\left\langle Q^{2}\right\rangle=7.093 \mathrm{GeV}^{2} \\
\left\langle t^{\prime}\right\rangle=-0.127 \mathrm{GeV}^{2}\end{array}$} & \multicolumn{2}{|c|}{$\begin{array}{c}\left\langle x_{B}\right\rangle=0.499 \\
\left\langle Q^{2}\right\rangle=7.115 \mathrm{GeV}^{2} \\
\left\langle t^{\prime}\right\rangle=-0.212 \mathrm{GeV}^{2}\end{array}$} & \multicolumn{2}{|c|}{$\begin{array}{c}\left\langle x_{B}\right\rangle=0.499 \\
\left\langle Q^{2}\right\rangle=7.106 \mathrm{GeV}^{2} \\
\left\langle t^{\prime}\right\rangle=-0.297 \mathrm{GeV}^{2}\end{array}$} & \multicolumn{2}{|c|}{$\begin{array}{c}\left\langle x_{B}\right\rangle=0.498 \\
\left\langle Q^{2}\right\rangle=7.102 \mathrm{GeV}^{2} \\
\left\langle t^{\prime}\right\rangle=-0.457 \mathrm{GeV}^{2}\end{array}$} \\
\hline 7.5 & $0.18 \pm 0.25$ & $\begin{array}{c}+0.00 \\
-0.10\end{array}$ & $0.60 \pm 0.35$ & $\begin{array}{l}+0.09 \\
-0.00\end{array}$ & $1.16 \pm 1.39$ & $\begin{array}{c}+0.39 \\
-0.00\end{array}$ & $-10.39 \pm 14.43$ & $\begin{array}{l}+0.00 \\
-0.03\end{array}$ & $0.00 \pm 0.00$ & $\begin{array}{l}+0.00 \\
-0.00\end{array}$ \\
\hline 22.5 & $0.59 \pm 0.25$ & $\begin{array}{l}+0.01 \\
-0.12\end{array}$ & $0.64 \pm 0.27$ & $\begin{array}{l}+0.09 \\
-0.03\end{array}$ & $-0.85 \pm 0.73$ & $\begin{array}{l}+0.13 \\
-0.13\end{array}$ & $-2.09 \pm 2.90$ & $\begin{array}{l}+0.00 \\
-0.00\end{array}$ & $0.00 \pm 0.00$ & $\begin{array}{c}+0.00 \\
-0.00\end{array}$ \\
\hline 37.5 & $0.45 \pm 0.23$ & $\begin{array}{l}+0.00 \\
-0.02\end{array}$ & $0.21 \pm 0.21$ & $\begin{array}{c}+0.00 \\
-0.03\end{array}$ & $-0.16 \pm 0.31$ & $\begin{array}{c}+0.06 \\
-0.07\end{array}$ & $0.00 \pm 0.65$ & $\begin{array}{c}+0.28 \\
-0.00\end{array}$ & $-1.16 \pm 1.79$ & $\begin{array}{l}+0.00 \\
-2.39\end{array}$ \\
\hline 52.5 & $0.26 \pm 0.21$ & $\begin{array}{c}+0.05 \\
-0.02\end{array}$ & $0.51 \pm 0.17$ & $\begin{array}{l}+0.01 \\
-0.04\end{array}$ & $0.85 \pm 0.19$ & $\begin{array}{l}+0.04 \\
-0.05\end{array}$ & $0.18 \pm 0.27$ & $\begin{array}{l}+0.05 \\
-0.00\end{array}$ & $0.50 \pm 0.20$ & $\begin{array}{l}+0.01 \\
-0.03\end{array}$ \\
\hline 67.5 & $0.24 \pm 0.21$ & $\begin{array}{l}+0.03 \\
-0.02\end{array}$ & $0.30 \pm 0.16$ & $\begin{array}{l}+0.01 \\
-0.05\end{array}$ & $0.64 \pm 0.15$ & $\begin{array}{l}+0.00 \\
-0.03\end{array}$ & $0.22 \pm 0.17$ & $\begin{array}{l}+0.01 \\
-0.01\end{array}$ & $0.20 \pm 0.09$ & $\begin{array}{c}+0.01 \\
-0.01\end{array}$ \\
\hline 82.5 & $0.21 \pm 0.19$ & $\begin{array}{c}+0.05 \\
-0.00\end{array}$ & $0.46 \pm 0.15$ & $\begin{array}{l}+0.00 \\
-0.07\end{array}$ & $0.41 \pm 0.13$ & $\begin{array}{c}+0.04 \\
-0.03\end{array}$ & $0.22 \pm 0.17$ & $\begin{array}{c}+0.02 \\
-0.04\end{array}$ & $0.37 \pm 0.07$ & $\begin{array}{c}+0.02 \\
-0.00\end{array}$ \\
\hline 97.5 & $0.29 \pm 0.18$ & $\begin{array}{l}+0.00 \\
-0.02\end{array}$ & $0.32 \pm 0.13$ & $\begin{array}{l}+0.02 \\
-0.00\end{array}$ & $0.25 \pm 0.12$ & $\begin{array}{l}+0.00 \\
-0.03\end{array}$ & $0.16 \pm 0.14$ & $\begin{array}{c}+0.01 \\
-0.03\end{array}$ & $0.16 \pm 0.06$ & $\begin{array}{l}+0.01 \\
-0.03\end{array}$ \\
\hline 112.5 & $0.29 \pm 0.17$ & $\begin{array}{l}+0.04 \\
-0.00\end{array}$ & $0.39 \pm 0.14$ & $\begin{array}{l}+0.02 \\
-0.04\end{array}$ & $0.11 \pm 0.11$ & $\begin{array}{l}+0.01 \\
-0.02\end{array}$ & $0.11 \pm 0.13$ & $\begin{array}{l}+0.01 \\
-0.04\end{array}$ & $0.16 \pm 0.05$ & $\begin{array}{l}+0.00 \\
-0.01\end{array}$ \\
\hline 127.5 & $0.44 \pm 0.16$ & $\begin{array}{l}+0.03 \\
-0.01\end{array}$ & $0.07 \pm 0.13$ & $\begin{array}{l}+0.05 \\
-0.00\end{array}$ & $0.22 \pm 0.10$ & $\begin{array}{l}+0.04 \\
-0.04\end{array}$ & $0.15 \pm 0.12$ & $\begin{array}{l}+0.01 \\
-0.05\end{array}$ & $0.17 \pm 0.05$ & $\begin{array}{l}+0.00 \\
-0.01\end{array}$ \\
\hline 142.5 & $0.20 \pm 0.16$ & $\begin{array}{l}+0.01 \\
-0.02\end{array}$ & $0.25 \pm 0.12$ & $\begin{array}{l}+0.02 \\
-0.00\end{array}$ & $0.35 \pm 0.11$ & $\begin{array}{l}+0.01 \\
-0.02\end{array}$ & $0.04 \pm 0.12$ & $\begin{array}{l}+0.02 \\
-0.02\end{array}$ & $0.01 \pm 0.05$ & $\begin{array}{c}+0.02 \\
-0.01\end{array}$ \\
\hline 157.5 & $0.20 \pm 0.16$ & $\begin{array}{l}+0.00 \\
-0.03\end{array}$ & $0.26 \pm 0.12$ & $\begin{array}{l}+0.00 \\
-0.00\end{array}$ & $0.36 \pm 0.10$ & $\begin{array}{l}+0.00 \\
-0.02\end{array}$ & $0.12 \pm 0.12$ & $\begin{array}{l}+0.00 \\
-0.01\end{array}$ & $0.04 \pm 0.05$ & $\begin{array}{l}+0.00 \\
-0.02\end{array}$ \\
\hline 172.5 & $-0.11 \pm 0.16$ & $\begin{array}{l}+0.01 \\
-0.04\end{array}$ & $0.06 \pm 0.11$ & $\begin{array}{l}+0.01 \\
-0.00\end{array}$ & $0.01 \pm 0.10$ & $\begin{array}{l}+0.02 \\
-0.00\end{array}$ & $-0.03 \pm 0.13$ & $\begin{array}{r}+0.02 \\
-0.00\end{array}$ & $0.02 \pm 0.05$ & $\begin{array}{l}+0.01 \\
-0.01\end{array}$ \\
\hline 187.5 & $0.23 \pm 0.15$ & $\begin{array}{c}+0.00 \\
-0.03\end{array}$ & $-0.07 \pm 0.11$ & $\begin{array}{c}+0.02 \\
-0.00\end{array}$ & $0.02 \pm 0.10$ & $\begin{array}{c}+0.01 \\
-0.06\end{array}$ & $0.15 \pm 0.11$ & $\begin{array}{c}+0.02 \\
-0.00\end{array}$ & $0.00 \pm 0.05$ & $\begin{array}{c}+0.00 \\
-0.00\end{array}$ \\
\hline 202.5 & $-0.12 \pm 0.16$ & $\begin{array}{c}+0.02 \\
-0.01\end{array}$ & $-0.15 \pm 0.11$ & $\begin{array}{c}+0.02 \\
-0.00\end{array}$ & $-0.01 \pm 0.10$ & $\begin{array}{c}+0.02 \\
-0.00\end{array}$ & $0.04 \pm 0.13$ & $\begin{array}{c}+0.03 \\
-0.00\end{array}$ & $0.00 \pm 0.05$ & $\begin{array}{c}+0.01 \\
-0.01 \\
\end{array}$ \\
\hline 217.5 & $-0.22 \pm 0.16$ & $\begin{array}{c}+0.00 \\
-0.02\end{array}$ & $-0.03 \pm 0.13$ & $\begin{array}{c}+0.01 \\
-0.01\end{array}$ & $-0.24 \pm 0.11$ & $\begin{array}{c}+0.01 \\
-0.03\end{array}$ & $0.02 \pm 0.12$ & $\begin{array}{c}+0.02 \\
-0.00\end{array}$ & $-0.06 \pm 0.05$ & $\begin{array}{l}+0.01 \\
-0.01\end{array}$ \\
\hline 232.5 & $-0.37 \pm 0.17$ & $\begin{array}{c}+0.02 \\
-0.03\end{array}$ & $-0.26 \pm 0.12$ & $\begin{array}{c}+0.05 \\
-0.01\end{array}$ & $-0.23 \pm 0.11$ & $\begin{array}{c}+0.02 \\
-0.06\end{array}$ & $-0.25 \pm 0.14$ & $\begin{array}{c}+0.01 \\
-0.04\end{array}$ & $-0.05 \pm 0.05$ & $\begin{array}{c}+0.00 \\
-0.01\end{array}$ \\
\hline 247.5 & $-0.22 \pm 0.17$ & $\begin{array}{c}+0.01 \\
-0.06\end{array}$ & $-0.26 \pm 0.13$ & $\begin{array}{c}+0.03 \\
-0.01\end{array}$ & $-0.13 \pm 0.11$ & $\begin{array}{c}+0.00 \\
-0.02\end{array}$ & $-0.38 \pm 0.14$ & $\begin{array}{c}+0.01 \\
-0.03 \\
\end{array}$ & $-0.23 \pm 0.05$ & $\begin{array}{c}+0.01 \\
-0.01 \\
\end{array}$ \\
\hline 262.5 & $-0.46 \pm 0.19$ & $\begin{array}{c}+0.07 \\
-0.06\end{array}$ & $-0.37 \pm 0.13$ & $\begin{array}{c}+0.01 \\
-0.02\end{array}$ & $-0.39 \pm 0.11$ & $\begin{array}{c}+0.00 \\
-0.01\end{array}$ & $-0.13 \pm 0.17$ & $\begin{array}{c}+0.02 \\
-0.00\end{array}$ & $-0.16 \pm 0.06$ & $\begin{array}{c}+0.01 \\
-0.00\end{array}$ \\
\hline 277.5 & $-0.48 \pm 0.20$ & $\begin{array}{c}+0.03 \\
-0.00\end{array}$ & $-0.38 \pm 0.14$ & $\begin{array}{c}+0.00 \\
-0.03\end{array}$ & $-0.26 \pm 0.14$ & $\begin{array}{c}+0.00 \\
-0.02\end{array}$ & $-0.12 \pm 0.16$ & $\begin{array}{c}+0.00 \\
-0.01\end{array}$ & $-0.18 \pm 0.07$ & $\begin{array}{c}+0.02 \\
-0.00\end{array}$ \\
\hline 292.5 & $-0.12 \pm 0.20$ & $\begin{array}{c}+0.01 \\
-0.03\end{array}$ & $-0.51 \pm 0.15$ & $\begin{array}{c}+0.05 \\
-0.00\end{array}$ & $-0.31 \pm 0.15$ & $\begin{array}{c}+0.01 \\
-0.00\end{array}$ & $-0.23 \pm 0.19$ & $\begin{array}{c}+0.03 \\
-0.00\end{array}$ & $-0.29 \pm 0.08$ & $\begin{array}{l}+0.01 \\
-0.01\end{array}$ \\
\hline 307.5 & $-0.07 \pm 0.22$ & $\begin{array}{c}+0.00 \\
-0.08\end{array}$ & $-0.52 \pm 0.18$ & $\begin{array}{c}+0.01 \\
-0.03\end{array}$ & $-0.10 \pm 0.19$ & $\begin{array}{c}+0.03 \\
-0.00\end{array}$ & $-0.55 \pm 0.30$ & $\begin{array}{c}+0.03 \\
-0.03\end{array}$ & $0.07 \pm 0.20$ & $\begin{array}{c}+0.03 \\
-0.18\end{array}$ \\
\hline 322.5 & $-0.68 \pm 0.26$ & $\begin{array}{c}+0.02 \\
-0.03\end{array}$ & $-0.04 \pm 0.22$ & $\begin{array}{c}+0.00 \\
-0.05\end{array}$ & $0.12 \pm 0.30$ & $\begin{array}{c}+0.00 \\
-0.04\end{array}$ & $-0.55 \pm 0.00$ & $\begin{array}{c}+0.16 \\
-0.00\end{array}$ & $-0.35 \pm 1.87$ & $\begin{array}{l}+0.01 \\
-0.03\end{array}$ \\
\hline 337.5 & $0.10 \pm 0.25$ & $\begin{array}{c}+0.00 \\
-0.05\end{array}$ & $-0.47 \pm 0.32$ & $\begin{array}{c}+0.18 \\
-0.01\end{array}$ & $0.01 \pm 0.68$ & $\begin{array}{c}+0.17 \\
-0.00\end{array}$ & $1.20 \pm 1.67$ & $\begin{array}{c}+0.00 \\
-0.00\end{array}$ & $-7.32 \pm 10.17$ & $\begin{array}{l}+1.07 \\
-0.00\end{array}$ \\
\hline 352.5 & $0.13 \pm 0.24$ & $\begin{array}{l}+0.02 \\
-0.05\end{array}$ & $-0.50 \pm 0.35$ & $\begin{array}{l}+0.13 \\
-0.05\end{array}$ & $0.95 \pm 0.59$ & $\begin{array}{c}+0.01 \\
-0.06\end{array}$ & $0.00 \pm 0.00$ & $\begin{array}{c}+0.00 \\
-0.00\end{array}$ & $0.00 \pm 0.00$ & $\begin{array}{c}+0.00 \\
-0.00\end{array}$ \\
\hline
\end{tabular}

Table E.4: Table of helicity-dependent extracted cross section values (pb/GeV ${ }^{4}$ ) for Kin484. The \pm values are the statistical errors, and the separated + and - are the systematic error introduced from the missing mass cut. The overall systematic uncertainties from table 6.4 are not represented here. 


\section{Appendix $\mathrm{F}$}

\section{Acceptance Cuts}

Below is a list of the $2 \mathrm{D}$ acceptance cuts for each kinematic setting in target variables. These are the 2D cuts used to define the R-Function (see section 3.4.3.1 for details about how these are used). 


\begin{tabular}{|l|}
\hline Kin 361,481 \\
\hline$\theta=-13.3 \delta p-0.55$ \\
$\theta=-4 \delta p+0.18$ \\
$\theta=-0.6 \delta p+0.07$ \\
$\phi=-0.2 \delta p+0.021$ \\
$\phi=0.125 \delta p-0.03$ \\
$\delta p=0.043$ \\
$\delta p=-0.04$ \\
$\phi=-0.27 y+0.026$ \\
$\phi=0.5775 y+0.042$ \\
$\phi=0.538 y-0.048$ \\
$\phi=-0.225 y-0.03$ \\
$\theta=0.06$ \\
$\theta=-0.05$ \\
$\phi=-0.028$ \\
$\phi=0.02$ \\
\hline
\end{tabular}

Table F.1: 2D Cuts for the Kinematic Settings 361 and 481

\begin{tabular}{|l|}
\hline Kin $362,363,601,603$ \\
\hline$\theta=-13.33 \delta p-0.56$ \\
$\theta=-0.574 \delta p+0.072$ \\
$\theta=-4.76 \delta p+0.219$ \\
$\phi=0.1 \delta p-0.032$ \\
$\phi=-0.22 y-0.031$ \\
$\phi=-0.12 y+0.028$ \\
$\theta=-0.05$ \\
$\theta=0.055$ \\
\hline
\end{tabular}

Table F.2: 2D Cuts for the Kinematic Settings 362, 363, 601, and 603 


\begin{tabular}{|l|}
\hline Kin 482 \\
\hline$\theta=-4 \delta p-0.17$ \\
$\theta=-0.4 \delta p+0.04$ \\
$\delta p=0.035$ \\
$\phi=-2.5 \delta p+0.0825$ \\
$\phi=0.1 \delta p-0.042$ \\
$\phi=-0.148 \delta p+0.025$ \\
$\phi=6.56 \delta p+0.24$ \\
$\phi=-0.075 y+0.0285$ \\
$\phi=-0.325 y-0.04$ \\
$\theta=0.0325$ \\
$\theta=-0.025$ \\
$\phi=-0.038$ \\
$\phi=0.015$ \\
\hline
\end{tabular}

Table F.3: 2D Cuts for the Kinematic Setting 482

\begin{tabular}{|l|}
\hline Kin 483 \\
\hline$\theta=-2.96 \delta p+0.137$ \\
$\theta=-0.492 \delta p+0.054$ \\
$\theta=-7.14 \delta p-0.293$ \\
$\phi=-4.2 \delta p+0.146$ \\
$\phi=0.1075 \delta p-0.038$ \\
$\phi=-7.14 \delta p-0.293$ \\
$\phi=-0.225 y+0.03$ \\
$\phi=-0.325 y-0.038$ \\
$\theta=0.05$ \\
$\theta=-0.036$ \\
$\phi=-0.037$ \\
$\phi=0.02$ \\
\hline
\end{tabular}

Table F.4: 2D Cuts for the Kinematic Setting 483 


\begin{tabular}{|l|}
\hline Kin 484 \\
\hline$\theta=-4.5 \delta p-0.19$ \\
$\theta=-0.45 \delta p+0.045$ \\
$\delta p=0.035$ \\
$\phi=-0.27 \delta p+0.015$ \\
$\phi=0.1 \delta p-0.035$ \\
$\phi=-1.4 \delta p+0.038$ \\
$\delta p=-0.03$ \\
$\phi=-0.217 y-0.032$ \\
$\phi=0.02$ \\
$\theta=-0.03$ \\
$\theta=0.038$ \\
$\phi=0.01$ \\
$\phi=0.03$ \\
\hline
\end{tabular}

Table F.5: 2D Cuts for the Kinematic Setting 484 


\section{Bibliography}

[1] R. W. McAllister and R. Hofstadter. Elastic Scattering of 199-Mev Electrons from the Proton and the Alpha Particle. Phys. Rev. 102, 851, 1956.

[2] E. D. Bloom et al. High-Energy Inelastic $e-p$ Scattering at $6^{\circ}$ and $10^{\circ}$. Phys. Rev. Lett. 23, 930, 1969.

[3] M. Breidnenbach et al. Observed Behavior of Highly Inelastic Electron-Proton Scattering. Phys. Rev. Lett. 23, 935, 1969.

[4] Murray Gell-Mann. A Schematic Model of Baryons and Mesons. Phys. Lett., 8, 214,1964.

[5] R. J. Hill. Review of experimental and theoretical status of the proton radius puzzle, 2017. arXiv:1702.01189.

[6] D. Muller, D. Robaschik, B. Geyer, F. M. Dittes, and J. Horejsi. Wave Functions, Evolution Equations and Evolution Kernels from Light-Ray Operators of QCD. Fortsch. Phys. 42, 101, 1994.

[7] N. Bezginov et al. A measurement of the atomic hydrogen Lamb shift and the proton charge radius. Science, 365, 1007, 2019

[8] A. Gasparian et al. PRad-II: A New Upgraded High Precision Measurement of the Proton Charge Radius, 2020. arXiv:2009.10510.

[9] D. L. Donoho. High-dimensional data analysis: The curses and blessings of dimensionality. https://citeseerx.ist.psu.edu/viewdoc/summary?doi=10.1.1.329.3392

[10] X. Ji. Gauge-Invariant Decomposition of Nucleon Spin and Its Spin-Off. Phys. Rev. Lett. 78, 610, 1996.

[11] X. Ji and J. Osborne. One loop corrections and all order factorization in deeply virtual Compton scattering. Phys. Rev. D 58, 094018, 1998.

[12] M. N. Rosenbluth. High Energy Elastic Scattering of Electrons on Protons. Phys. Rev. 79, 615, 1950. 
[13] J. J. Kelly. Simple parametrization of nucleon form factors. Phys. Rev. C 70, 068202, 2004.

[14] J. C. Bernauer et al. The electric and magnetic form factors of the proton. Phys. Rev. Lett. 105, 242001, 2010.

[15] R. Pohl et al. The size of the proton. Nature 466, 213, 2010.

[16] X. Ji. Deeply-Virtual Compton Scattering, 1997. arXiv: 9609381v3.

[17] A. V. Belitsky, D. Muller, Y. Ji. Compton scattering: from deeply virtual to quasi-real, 2014. arXiv: $1212.6674 v 2$.

[18] H. W. Kendall. Deep Inelastic Scattering: Experiments on the proton and the observation of sclaing. Rev. Mod. Phys. 63, 597, 1991.

[19] R. P. Feynman. Very High-Energy Collisions of Hadrons. Phys. Rev. Lett. 23, 1415, 1969.

[20] K. A. Olive et al. Review of Particle Physics. Chin. Phys., C38:090001, 2014.

[21] X. Ji and J. Osborne. One loop corrections and all order factorizations in deeply virtual Compton scattering. Phys. Rev. D 58, 094018, 1998.

[22] J. C. Collins and A. Freund. Proof of factorization for deeply virtual Compton scattering in QCD. Phys. Rev. D 59, 074009, 1999.

[23] N. d'Hose, S. Niccolai, and A. Rostomyan. Experimental overview of Deeply Virtual Compton Scattering. Eur. Phys. J. A 52, 151, 2016.

[24] N. d?Hose, S. Niccolai, and A. Rostomyan. Experimental overview of Deeply Virtual Compton Scattering. Eur. Phys. J. A 52, 151, 2016.

[25] C. Adloff et al. (H1 Collaboration). Measurement of Deeply Virtual Compton Scattering at HERA. Phys. Lett. B 517, 47-58, 2001. hep-ex/0107005.

[26] S. Chekanov et al. (ZEUS Collaboration). Measurement of Deeply Virtual Compton Scattering at HERA. Phys. Lett. B 573, 46-62, 2003. hep-ex/0305028.

[27] A. Aktas et al. (H1 Collaboration). Measurement of Deeply Virtual Compton Scattering at HERA. Eur. Phys. J. C 44 1-11, 2005. hep-ex/0505061.

[28] F. D. Aaron et al. (H1 Collaboration). Measurement of Deeply Virtual Compton Scattering and its t-dependence at HERA. Phys. Lett. B 659, 796-806, 2007. arXiv:0709.4114.

[29] S. Chekanov et al. (ZEUS Collaboration). A measurement of the $Q^{2}, \mathrm{~W}$ and t dependencies of deeply virtual Compton scattering at HERA. JHEP 0905:108, 2007. arXiv:0812.2517. 
[30] F. D. Aaron et al. (H1 Collaboration). Deeply Virtual Compton Scattering and its Beam Charge Asymmetry in $\mathrm{e}^{ \pm} \mathrm{p}$ Collisions at HERA. JHEP 0905:108, 2009. arXiv:0812.2517.

[31] A. Airapetian et al. (HERMES Collaboration). Measurement of the Beam-Spin Azimuthal Asymmetry Associated with Deeply Virtual Compton Scattering. Phys. Rev. Lett. 87, 182001, 2001. hep-ex/0106068.

[32] A. Airapetian et al. (HERMES Collaboration). Beam-helicity asymmetry arising from deeply virtual Compton scattering measured with kinematically complete event reconstruction. JHEP 10, 042, 2012. arXiv:1206.5683.

[33] A. Airapetian et al. (HERMES Collaboration). The Beam-Charge Azimuthal Asymmetry and Deeply Virtual Compton Scattering. Phys. Rev. D 75, 011103, 2007. hepex/0605108.

[34] A. Airapetian et al. (HERMES Collaboration). Separation of contributions from deeply virtual Compton scattering and its interference with the Bethe-Heitler process in measurements on a hydrogen target. JHEP 0911:083, 2009. arXiv:0909.3587.

[35] A. Airapetian et al. (HERMES Collaboration). Beam-helicity and beam-charge asymmetries associated with deeply virtual Compton scattering on the unpolarized proton. JHEP 0\%, 032, 2012.

[36] A. Airapetian et al. (HERMES Collaboration). Exclusive Leptoproduction of Real Photons on a Longitudinally Polarized Hydrogen Target. JHEP 1006:019, 2010. arXiv:1004.0177.

[37] A. Airapetian et al. (HERMES Collaboration). Measurement of azimuthal asymmetries associated with deeply virtual Compton scattering on a longitudinally polarized deuterium target. Nucl. Phys. D 842, 265-298, 2011. arXiv:1008.3996.

[38] A. Airapetian et al. (HERMES Collaboration). Measurement of Azimuthal Asymmetries With Respect To Both Beam Charge and Transverse Target Polarization in Exclusive Electroproduction of Real Photons. JHEP 0806, 066, 2008. arXiv:0802.2499.

[39] F. Gautheron et al. (COMPASS Collaboration). COMPASS-II Proposal. CERN/SPSC-2010-014, SPSC-P-340, 2010.

[40] A. Ferrero et al. (COMPASS Collaboration). GPD Physics With Polarized Muon Beams at COMPASS-II. AIP Conference Proceedings 1523, 2013, 75.

[41] S. Stepanyan et al. (CLAS Collaboration). Observation of exclusive DVCS in polarized electron beam asymmetry measurements. Phys. Rev. Lett. 87, 182002, 2001. hepex/0107043. 
[42] F. X. Girod et al. (CLAS Collaboration). Deeply Virtual Compton Scattering BeamSpin Asymmetries. Phys. Rev. Lett. 100, 162002, 2008. arXiv:0711.4805.

[43] H. S. Jo et al. (CLAS Collaboration). Cross sections for the exclusive photon electroproduction on the proton and Generalized Parton Distributions. Phys Rev. Lett. 115, 212003, 2015. arXiv:1504.02009.

[44] S. Pisano et al. (CLAS Collaboration). Single and double spin asymmetries for deeply virtual Compton scattering measured with CLAS and a longitudinally polarized proton target. Phys. Rev. D 91, 052014, 2015. arXiv:1501.07052.

[45] E. Seder et al. (CLAS Collaboration). Longitudinally target-spin asymmetries for deeply virtual Compton scattering. Phys. Rev. Lett. 114, 032001, 2015. arXiv:1410.6615.

[46] A. Accardi et al. White paper: Electron Ion Collider: The Next QCD Frontier Understanding the glue that binds us all, 2014. arXiv:1212.1701.

[47] R. Dupre et al. Analysis of Deeply Virtual Compton Scattering Data at Jefferson Lab and Proton Tomography, 2017. arXiv:1704.07330.

[48] J. Roche, C. E. Hyde-Wright, B. Michel, C. Muñoz Camacho et al. (Jefferson Lab Hall A Collaboration). Measurements of the electron-helicity dependent cross sections of Deeply Virtual Compton Scattering with CEBAF at $12 \mathrm{GeV}$. PR12-06114, 2006. http://hallaweb.jlab.org/experiment/DVCS/documents/proposals/E12-06114_proposal.pdf.

[49] M. Defurne et al. (Jefferson Lab Hall A Collaboration). The E00-110 experiment in Jefferson Lab?s Hall A: Deeply Virtual Compton Scattering off the Proton at $6 \mathrm{GeV}$. Phys. Rev. C 92, 055202, 2015. arXiv:1504.05453.

[50] C. Muñoz Camacho et al. (Jefferson Lab Hall C Collaboration). Exclusive Deeply Virtual Compton and Neutral Pion Cross-Section Measurements in Hall C. PR12-13010, 2013. https://www.jlab.org/exp_prog/proposals/13/PR12-13-010.pdf.

[51] F. Sabatie et al. (CLAS12 Collaboration). Deeply Virtual Compton Scattering with CLAS at 11 GeV. PR12-06-119, 2006. https://www.jlab.org/exp_prog/proposals/06/PR12-06-119.pdf.

[52] S. Niccolai et al. (CLAS12 Collaboration). Deeply Virtual Compton Scattering on the Neutron with CLAS12 at 11 GeV. PR12-11-003, 2011. https://www.jlab.org/exp_prog/proposals/11/PR12-11-003.pdf.

[53] S. Niccolai et al. (CLAS12 Collaboration). Proposal of extension of the CLAS12 run-group Cb (ND3 target). C12-15-004, 2016. https://www.jlab.org/exp_prog/proposals/16/C12-15-004.pdf. 
[54] L. Cardman et al. White paper: The science driving the $12 \mathrm{GeV}$ upgrade of CEBAF, February 2001. https://www.jlab.org/div_dept/physics_division/GeV/whitepaperv11/index.html.

[55] J. Alcorn, B.D. Anderson, K.A. Aniol, J.R.M. Annand, L. Auerbach, et al. Basic Instrumentation for Hall A at Jeffrson Lab. Nucl. Instrum. Meth., A522:294-346, 2004.

[56] A. V. Belitsky, D. Muller, and A. Kirchner. Theory of deeply virtual Compton scattering on the nucleon. Nucl. Phys. B 629, 323-392, 2002. arXiv:hep-ph/0112108

[57] A. V. Belitsky and D. Muller. Exclusive electroproduction revisited: treating kinematical effects. Phys. Rev. D. 82, 074010, 2010.

[58] A. V. Belitsky, D. Muller, and Y. Ji. Compton scattering: from deeply virtual to quasi-real. Nucl. Phys. B878, 214-268, 2014. arXiv:1212.6674.

[59] W. Bertozzi et al. Precision Measurement of the Proton Elastic Cross Section at High $Q^{2}$. https://www.jlab.org/exp_prog/proposals/07/PR12-07-108.pdf

[60] M. E. Christy and P. E. Bosted, Phys. Rev. C 81, 055213 (2010).

[61] F. Georges. Internal note, 2018. https://hallaweb.jlab.org/dvcslog/12+GeV/494

[62] L. L. Frankfurt et al. Hard Exclusive Electroproduction of Decuplet Baryons in the Large Nc Limit. Phys. Rev. Lett. 84, 2589, 2000.

[63] F. Georges. Deeply Virtual Compton Scattering at Jefferson Lab. October 2018. PhD Dissertation.

[64] M. Defurne. Measurement of Hard Exclusive Electroproduction of $\pi^{0}$ Meson Cross Section at JLab with CEBAF at $12 \mathrm{GeV}$. June 2015. PhD Dissertation.

[65] M. Defurne et al. (Jefferson Lab Hall A Collaboration). The E00-110 experiment in Jefferson Lab's Hall A: Deeply Virtual Compton Scattering off the Proton at $6 \mathrm{GeV}$. Phys. Rev. C 92, 055202, 2015. arXiv:1504.05453.

[66] M. Mazouz et al. (Jefferson Lab Hall A Collaboration) Proposal: Measurement of the Deeply Virtual Compton Scattering cross-section off the neutron.

[67] C. Camacho, 2013. PAC Presentation: Exclusive Deeply Virtual Compton Scattering and $\pi^{0}$ Cross Section Measurements in Hall C.

[68] L.W. Mo and Y. S. Tsai. Radiative corrections to elastic and inelastic ep and up scattering. Rev. Mod. Phys. 41, 205-235, 1969.

[69] M. Vanderhaeghen et al. QED radiative corrections to virtual Compton scattering. Phys. Rev. C 62, 025501, 2000. arXiv:hep-ph/0001100. 
[70] C. E. Hyde-Wright. Comment on Radiative Corrections in Virtual Compton Scattering, 2006. https://hallaweb.jlab.org/dvcslog/DVCS2/235.

[71] C. E. Hyde-Wright. Non-Factorized Form of Radiative Coorections, 2015. https://hallaweb.jlab.org/dvcslog/Results/189.

[72] R. T. Jones et al. A bootstrap method for gain calibration and resolution determination of a lead-glass calorimeter. Nuclear Instruments and Methods in Physics Research A $566366-374,2006$.

[73] B. Karki. Deep Exclusive $1^{0}$ Electroproduction Measured in Hall A at Jefferson Lab with the Upgraded CEBAF. August 2020. PhD Dissertation.

[74] M. Defurne et al. A glimpse of gluons through deeply virtual compton scattering on the proton. Nature Communications 8: 1408, 2017. https://www.nature.com/articles/s41467-017-01819-3.pdf

[75] Kumericki, K., Liuti, S. \& Moutarde, H. GPD phenomenology and DVCS fitting. Eur. Phys. J. A 52, 157 (2016).

[76] K. Kumericki and D. Muller. Description and interpretation of DVCS measurements. EPJ Web of Conferences 112, 01012, 2015. arXiv:1512.09014.

[77] Dupre, R., Guidal, M. \& Vanderhaeghen, M. Tomographic image of the proton. Phys. Rev. D 95, 011501 (2017). 